Scientific Committee on Emerging and Newly Identified Health Risks

SCENIHR

Opinion on

The safety of the use of bisphenol $A$ in medical devices

\title{
Scientific Committees
}

- on consumer safety

on emerging and newly identified health risks

- on health and environmental risks

SCENIHR adopted this opinion by written procedure on 18 February 2015 


\section{About the Scientific Committees}

Three independent non-food Scientific Committees provide the Commission with the scientific advice it needs when preparing policy and proposals relating to consumer safety, public health and the environment. The Committees also draw the Commission's attention to the new or emerging problems which may pose an actual or potential threat.

They are: the Scientific Committee on Consumer Safety (SCCS), the Scientific Committee on Health and Environmental Risks (SCHER) and the Scientific Committee on Emerging and Newly Identified Health Risks (SCENIHR).

In addition, the Commission relies upon the work of the European Food Safety Authority (EFSA), the European Medicines Agency (EMA), the European Centre for Disease prevention and Control (ECDC) and the European Chemicals Agency (ECHA).

\section{SCENI HR}

This Committee deals with questions related to emerging or newly identified health and environmental risks and to broad, complex or multidisciplinary issues requiring a comprehensive assessment of risks to consumer safety or public health and related issues not covered by other Community risk assessment bodies. Examples of potential areas of activity include potential risks associated with interaction of risk factors, synergic effects, cumulative effects, antimicrobial resistance, new technologies such as nanotechnologies, medical devices including those incorporating substances of animal and/or human origin, tissue engineering, blood products, fertility reduction, cancer of endocrine organs, physical hazards such as noise and electromagnetic fields (from mobile phones, transmitters and electronically controlled home environments), and methodologies for assessing new risks. It may also be invited to address risks related to public health determinants and non-transmissible diseases.

\section{Scientific Committee members}

Michelle Epstein, Igor Emri, Philippe Hartemann, Peter Hoet, Norbert Leitgeb, Luis Martínez Martínez, Ana Proykova, Luigi Rizzo, Eduardo Rodriguez-Farré, Lesley Rushton, Konrad Rydzynski, Theodoros Samaras, Emanuela Testai, Theo Vermeire

\section{Contact:}

\section{European Commission}

DG Health and Food Safety

Directorate C Public Health

Unit C2 - "Health Information and Scientific Committees"

Office: HTC 03/073 L-2920 Luxembourg

SANTE-C2-SCENIHR@ec.europa.eu

(C) European Commission 2015

ISSN $1831-4783$

Doi: $10.2772 / 75546$
ISBN 978-92-79-30133-9

ND-AS-13-003-EN-N

The opinions of the Scientific Committees present the views of the independent scientists who are members of the committees. They do not necessarily reflect the views of the European Commission. The opinions are published by the European Commission in their original language only.

http://ec.europa.eu/health/scientific committees/emerging/opinions/index en.htm 


\section{ACKNOWLEDGMENTS}

Members of the working group are acknowledged for their valuable contribution to this Opinion. They are:

\section{SCENIHR members:}

Dr. Emanuela Testai - Istituto Superiore di Sanità, Environment \& Primary Prevention Dept., Mechanisms of Toxicity Unit, Roma, Italy. Chair of the Working Group since April 2013.

Prof. Philippe Hartemann - Université de Lorraine, Faculté de Médecine, Nancy, France.

Prof. Eduardo Rodriguez-Farré - Barcelona Institute of Biomedical Research, Barcelona, Spain.

\section{SCCS member:}

Dr. Suresh Chandra Rastogi.

\section{External experts:}

Dr. Wim De Jong - National Institute for Public Health and the Environment (RIVM), Bilthoven, the Netherlands. Chair of the Working Group until March 2013 and rapporteur.

Dr. Juana Bustos - National Food Centre - Spanish Food Safety and Nutrition Agency (AESAN), Spain.

Dr. Laurence Castle - FERA, York, United Kingdom.

Prof. Ursula Gundert-Remy - Medical School (Charité), Berlin, Germany.

Prof. Arne Hensten- UiT The Arctic University of Norway, Tromsö, Norway.

Dr. Hilde Molvig Kopperud - Nordic Institute of Dental Materials, Oslo, Norway.

Prof. Nicolás Olea - University of Granada, Granada, Spain.

Prof. Aldert Piersma - National Institute for Public Health and the Environment, Bilthoven, the Netherlands.

All Declarations of working group members and supporting experts are available at the following webpage:

http://ec.europa.eu/health/scientific committees/emerging/members wg/index en.htm 


\section{ABSTRACT}

Bisphenol A (BPA) is a key building block of polycarbonate plastic and a precursor for the manufacturing of monomers of epoxy resins. The BPA exposure of the general population is via food as a result of the use of BPA in food packaging and via skin as a result of contact with thermal paper. The vast majority of the population (91-99\%) has detectable levels of BPA-conjugates in their urine. BPA is also present in medical devices including implants, catheters, tubing, and some dental materials. This Opinion describes the risk assessment of exposure to BPA via medical devices that are manufactured with materials that potentially leach BPA leading to oral (via dental material), subcutaneous and intravenous (e.g. during heamodialysis) routes of exposure.

After oral exposure BPA is readily absorbed from the gastro-intestinal tract and due to the first pass effect in the liver and the small intestine is rapidly conjugated to non-toxic metabolites. By the oral route BPA has a low systemic bioavailability (1-10\% in humans) and has a half life time of a few hours. For parenteral routes of exposure (intravenous, intraperitoneal, subcutaneous), BPA can be considered $100 \%$ systemically bioavailable. However, BPA will also be conjugated in the liver and the clearance of free BPA from the circulation appears to be relatively fast.

Toxicity studies indicate that the kidney and the liver are relevant target organs for BPA toxicity. The lowest NOAEL after oral repeated exposure identified in several studies, including multigeneration reproductive toxicity studies, was approximately $5 \mathrm{mg} / \mathrm{kg}$ b.w./day. By applying the benchmark dose (BMD) approach, a BMDL ${ }_{10}$ of $8.96 \mathrm{mg} / \mathrm{kg}$ b.w./day was derived (EFSA 2015), based on the alteration in kidney weight. BPA is not likely to pose a genotoxic hazard to humans and has no carcinogenic activity, although there are some effects observed in the mammary gland, which currently are of unknown significance to human health. Neither reproductive nor prenatal developmental toxicity are critical end-points in BPA toxicity, though BPA is associated with reproductive toxicity at doses higher than those causing liver and kidney damage.

There are several indications that BPA might have biological effects below the recently determined $\mathrm{BMDL}_{10}$. However, the evidence is inconsistent, mainly obtained in dedicated studies focussing on different selected health effects and dose-response relationships could not be established. Regarding possible low dose effects, some concern remains for effects on mammary gland, metabolism and adiposity and neurobehaviour.

The SCENIHR adopts the temporary oral TDI (t-TDI) of $4 \mu \mathrm{g} / \mathrm{kg} \mathrm{b.w./day} \mathrm{derived} \mathrm{by}$ EFSA which considers the $\mathrm{BMDL}_{10}$ as point of departure, and the uncertainties related to some effects using a BPA-specific assessment factor of 150 . This $t-T D I$ represents a useful base for carrying out a BPA risk assessment for the use of BPA in medical devices.

The $\mathrm{BMDL}_{10}$ dose was translated into a human dose inducing similar effects, the human equivalent dose (HED). The HED of $609 \mu \mathrm{g} / \mathrm{kg}$ b.w./day was determined considering the ratio of internal exposure in mice (the species used in the study from which $B M D L_{10}$ was derived) versus the internal exposure in humans based on toxicokinetic studies.

For medical devices, several exposure scenarios were evaluated taking into account the material used, information related to BPA leaching, duration of a single treatment and the frequency of treatments, giving rise to toxicologically relevant acute, short and long term exposure. The information available is very limited and in many cases due to the lack of experimental data, only estimations were used. The uncertainties related to the exposure assessment indeed represent the weakest part of this evaluation. For this reason only the highest value obtained in the estimate was indicatively used.

The estimated BPA exposures were: 1) $3000 \mathrm{ng} / \mathrm{kg}$ b.w./day (3 $\mu \mathrm{g} / \mathrm{kg}$ b.w./day) for prematurely born infants in neonatal intensive care units; 2) $685 \mathrm{ng} / \mathrm{kg}$ b.w./day for prolonged medical procedures (such as extracorporeal circulation or transplantation/ implantation of an artificial organ) in infants (b.w. around $5 \mathrm{~kg}$ ); 3) $57 \mathrm{ng} / \mathrm{kg} \mathrm{b.w./day}$ for dialysis patients; 4) 0.4 - $12 \mathrm{ng} / \mathrm{kg}$ b.w./day for long-term exposures to medical devices; 5) 140 to $200 \mathrm{ng} / \mathrm{kg}$ b.w./day for, respectively, children and adults due to 
contact with dental materials $(<24 \mathrm{~h}) ; 6) 2$ to $12 \mathrm{ng} / \mathrm{kg}$ b.w./day due to long-term contact with dental materials.

Exposure via medical devices consisting of BPA containing PVC might even be higher with values estimated for adults up to $5000 \mathrm{ng} / \mathrm{kg} \mathrm{b.w./day} \mathrm{and} \mathrm{infants} \mathrm{up} \mathrm{to} 12000 \mathrm{ng} / \mathrm{kg}$ b.w./day. However, exposure to BPA via BPA-containing PVC has been estimated based on extrapolation from data on phthalate leakage from PVC and has a high degree of uncertainty. Notably, European PVC manufacturers informed the SCENIHR that they no longer use BPA in PVC production, resulting in an expected low level of exposure, BPAcontaining medical devices from extra-EU manufacturers may be available in Europe.

Long-term oral exposure to BPA via dental material is below the t-TDI of $4 \mu \mathrm{g} / \mathrm{kg}$ b.w./day, thus posing a negligible risk for human health.

Regarding the parenteral exposure via medical devices, the kinetic differences between routes of exposure indicate that the bioavailability after oral route of exposure is significantly lower (around $1 \%$ ) when compared to the parenteral one (assumed as $100 \%)$. Therefore, the SCENIHR applied a margin of safety (MOS) approach using an internal dose $(6 \mu \mathrm{g} / \mathrm{kg}$ b.w./day $=\mathrm{HED} / 100)$ rather than the external t-TDI.

Applying a MOS approach, for the $3 \mu \mathrm{g} / \mathrm{kg}$ b.w./day corresponding to medical devices use in intensive care the MOS is 2; for repeated medical procedures in infants the MOS is 10; for the other short term exposure scenarios estimated for different medical devices, the MOS ranges between 43 and 100; for dialysis treatments the MOS is 105 . For the other scenarios the exposure ranges from $0.4 \mathrm{ng} / \mathrm{kg}$ b.w./day to $12 \mathrm{ng} / \mathrm{kg} \mathrm{b.w./day}$ resulting in a MOS range from 500 to 15,000 .

The uptake by the oral mucosa can be considerable: assuming a worst case scenario as $100 \%$, for the highest exposures $200 \mathrm{ng} / \mathrm{kg}$ b.w./day the MOS would be 30 . However, the peak of exposure occurs for $<24$ hours (representative of acute exposure in a toxicological context), whereas the MOS of 150 is related to chronic exposure. The differences in exposure duration can be larger than 5, therefore a MOS of 30 for acute exposure to dental materials can be considered sufficiently large.

Duration of exposure is indeed very relevant. With the exception of dialysis patients for which treatment is repeated for long time periods and can be considered similar to chronic exposure, the exposure via medical devices is generally of limited duration (representative of acute to subchronic exposure in a toxicological context). The application of correction factors to the MOS accounting for the time of exposure ( 3 as used by ECHA) might indicate that MOS around 50 could be sufficiently large, although with a high degree of uncertainties. Nevertheless some scenarios still raise concern.

The SCENIHR concludes that risk for adverse effects of BPA may exist when the BPA is directly available for systemic exposure after non-oral exposure routes, especially for neonates in intensive care units, infants undergoing prolonged medical procedures and for dialysis patients. Although the benefit of medical devices has also to be considered, the SCENIHR recommends that, where practicable, medical devices that do not leach BPA should be used. The possibility of replacing BPA in these products should be considered against their efficiency in the treatment, as well as the toxicological profile of the alternative materials.

However, better data on exposure would be beneficial for the refinement of the present risk assessment, to be carried out when new data on exposure via medical devices become available.

Keywords:

Keywords: Bisphenol A, risk assessment, safety, medical devices, SCENIHR.

SCENIHR (Scientific Committee on Emerging and Newly Identified Health Risks), Safety of the use of bisphenol A in medical devices, 18 February 2015 


\section{TABLE OF CONTENTS}

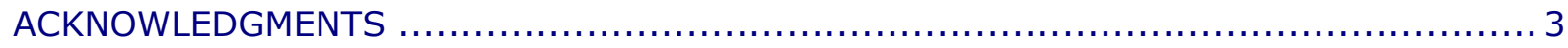

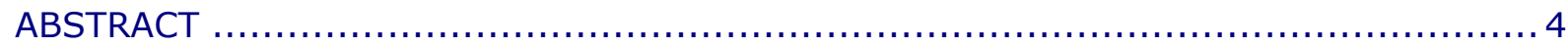

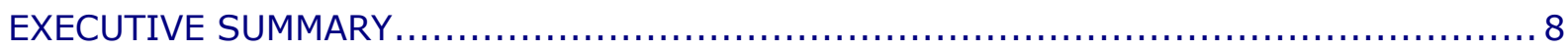

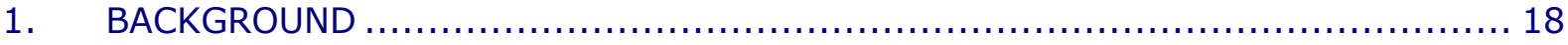

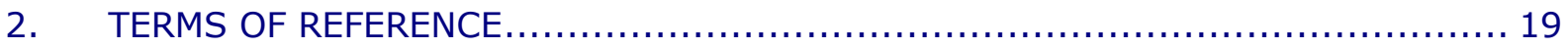

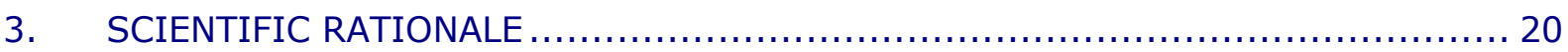

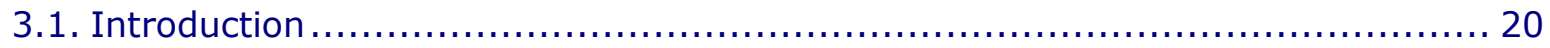

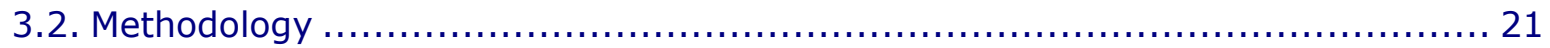

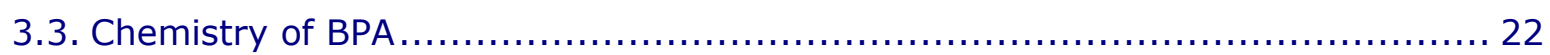

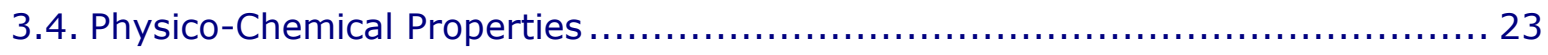

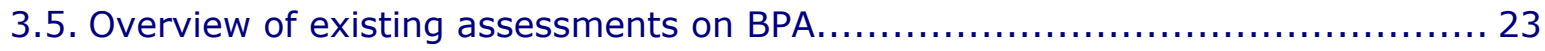

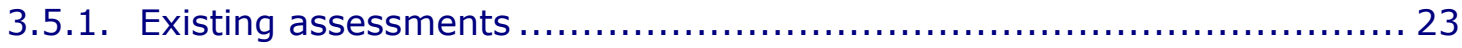

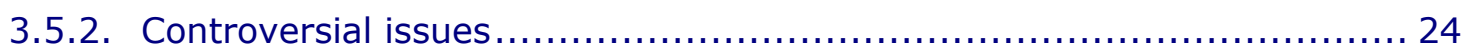

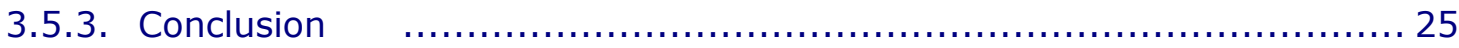

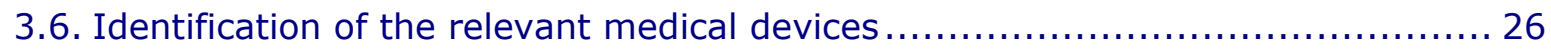

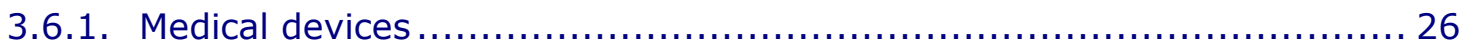

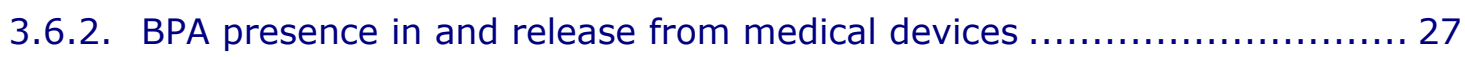

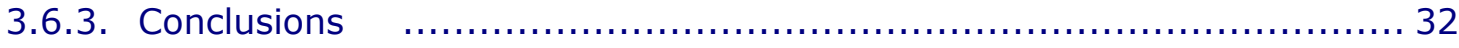

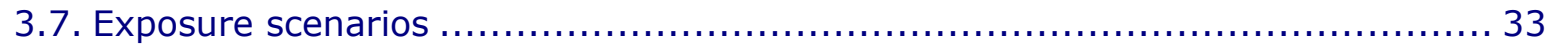

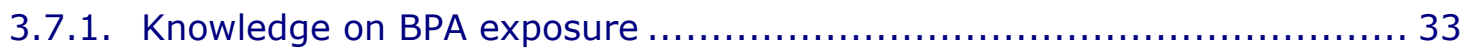

3.7.1.1. Methods for measurement of internal exposure in humans .... 33

3.7.1.2. Internal exposure to BPA in humans from all routes ............ 35

3.7.1.3. Non-oral exposure routes ................................... 40

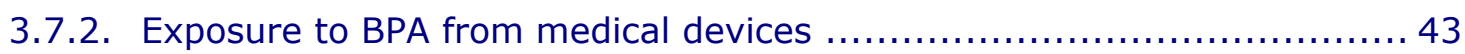

3.7.3. Exposure to BPA from medical devices under different scenarios ........... 47

3.7.4. BPA exposure from uses of BPA containing PVC .......................... 51

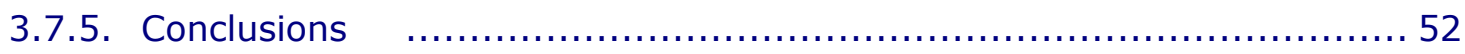

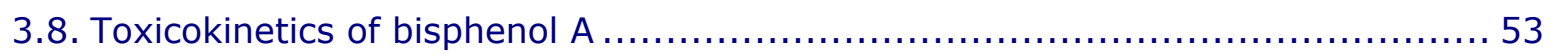

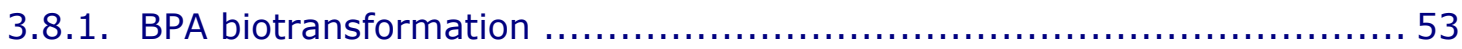

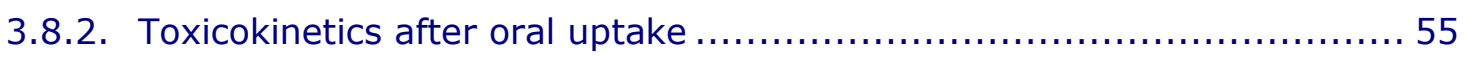

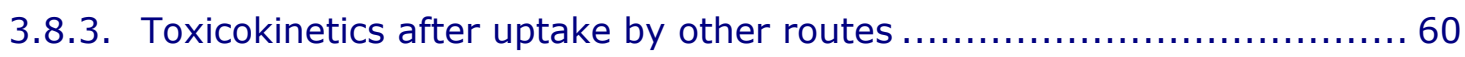

3.8.3.1. Toxicokinetics after dermal and transcutaneous uptake........60 60

3.8.3.2. Toxicokinetics after intravenous administration ................6 64

3.8.3.3. Toxicokinetics after inhalation .............................. 65

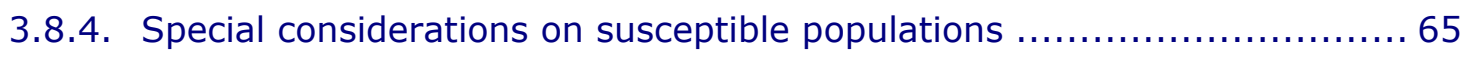

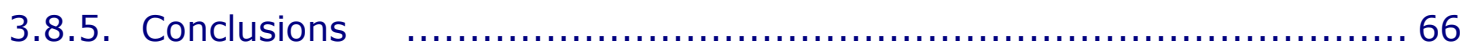

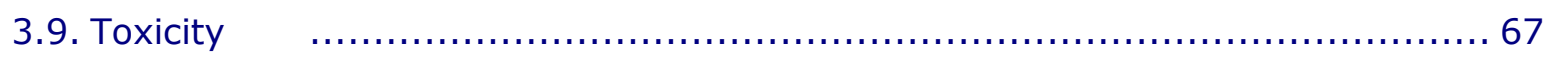

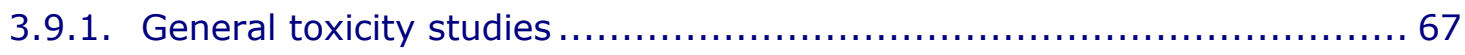




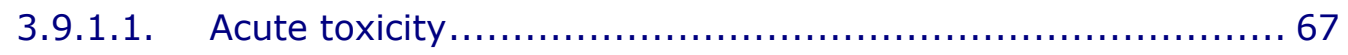

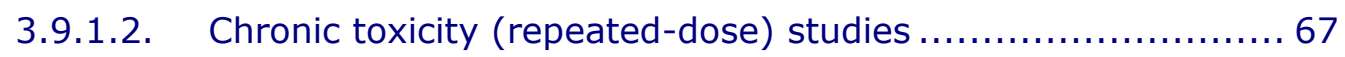

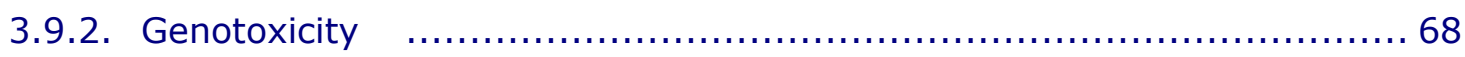

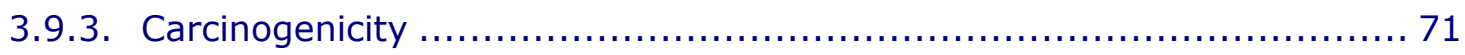

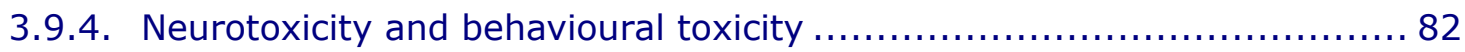

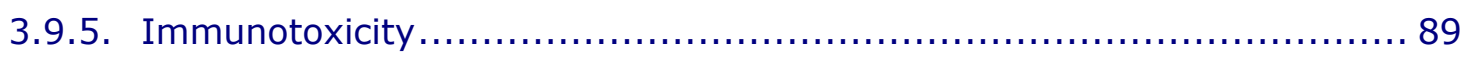

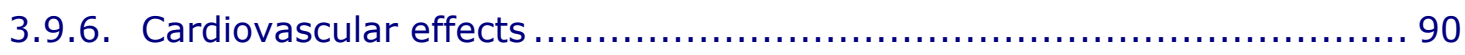

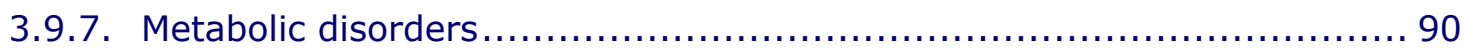

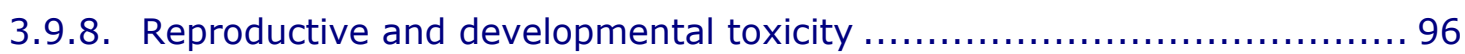

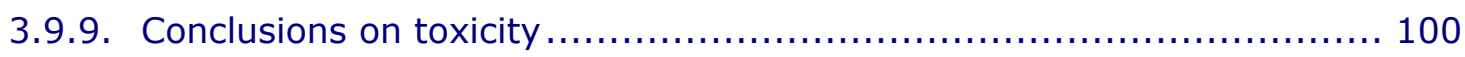

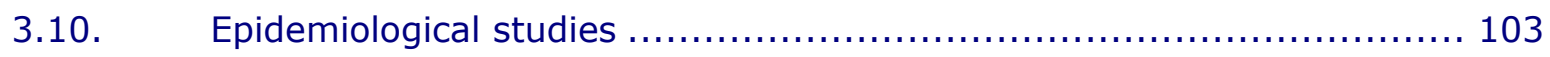

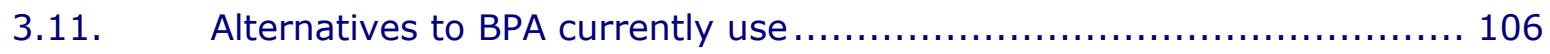

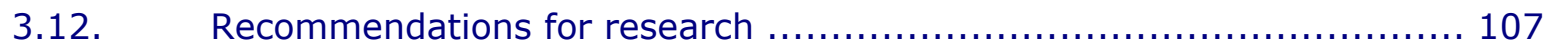

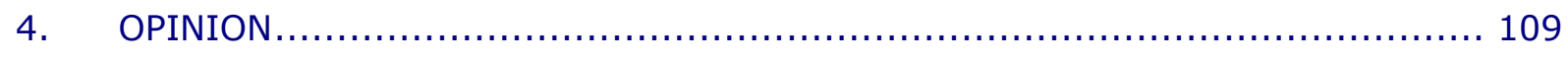

5. CONSIDERATION OF THE RESPONSES RECEIVED DURING THE CONSULTATION

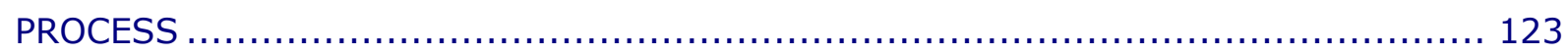

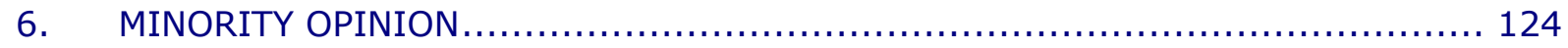

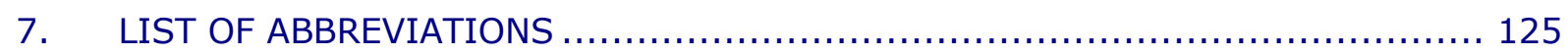

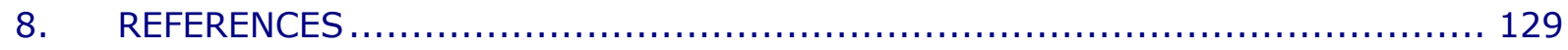




\section{EXECUTI VE SUMMARY}

\section{Background}

Bisphenol A [Bis(4-hydroxyphenyl)propane] (BPA) is a high-production volume industrial chemical, being a key building block of polycarbonate (PC) plastic and a precursor for the manufacturing of monomers of epoxy resins. Currently, many scientific discussions are ongoing on possible adverse effects of BPA. The general public is mostly exposed to BPA via food as a result of the use of BPA in food packaging and via skin as a result of contact with thermal paper. Polycarbonate is used in medical devices production (e.g. implants, catheters, tubing) for its balance of toughness, dimensional stability optical clarity, high heat resistance and electrical resistance. They may contain and release residual BPA during actual condition of use. In addition to PC medical devices, various dental materials are fabricated from monomers such as bisphenol A glycidyl methacrylate (Bis-GMA) and bisphenol A dimethacrylate (Bis-DMA), derived from BPA. BPA-resins are also used in inks and adhesives. In addition to BPA itself, polymers produced using BPA like polysulfone (PSU) used in medical devices (e.g. membrane in hemolysis dialyzers) are considered because they can release BPA. Some BPA-containing medical devices may have direct and/or indirect contact with patients (e.g. hemodialyzer apparatus, filters, bypasses, tubing, pumps, instruments, surgical equipment, blood pathway circuits and respiratory tubing circuits). This Opinion describes the risk assessment of exposure to BPA via medical devices made up of materials potentially leaching BPA, for which the exposure routes are not limited to oral applications (i.e. for dental material) as exposure via other applications such as subcutaneous and intravenous (e.g. during haemodialysis) routes may occur.

\section{Previous risk assessments}

In the previous evaluations, the following conclusions have been drawn for the oral route of exposure to BPA:

- No Observed Adverse Effect Level (NOAEL) of $5 \mathrm{mg} / \mathrm{kg} \mathrm{b.w./day} \mathrm{in} \mathrm{rats}$

- Tolerable Daily Intake (TDI) of $50 \mu \mathrm{g} / \mathrm{kg}$ body weight

- developmental toxic effects only observed at doses with severe maternal toxicity in rats and mice

- an overall NOAEL for reproductive toxicity of $50 \mathrm{mg} / \mathrm{kg} \mathrm{b} . \mathrm{w} . /$ day in rats and mice

- in terms of toxicokinetics, there is a difference between rats and humans (the latter presenting a shorter half-life) as well as between the oral and the parenteral route of exposure.

- due to the first pass effect, after oral uptake, the systemic exposure to free BPA is a small fraction of the external dose in all species.

- there are still unresolved issues in the risk assessment of BPA after oral uptake.

Very recently, EFSA established a temporary (t)-TDI of $4 \mu \mathrm{g} / \mathrm{kg}$ b.w./day for oral exposure to BPA based on kidney alterations as the critical effect (EFSA 2015). EFSA (2015) also included the dermal route of exposure in its latest evaluation.

For medical devices manufactured from PC plastics, parenteral routes such as subcutaneous and intravenous (e.g. during haemodialysis) are also important.

\section{General exposure}

The human population is exposed to BPA through the diet, while air, dust, water, and skin contact with thermal paper are other possible sources of exposure. Bisphenol $A$ in food and beverages accounts for about $50 \%$ of daily human internal exposure whereas skin contact with BPA-containing thermal paper is the source for the another $50 \%$.of internal exposure of the general population. BPA exposure results from either the release of non-polymerized monomers or from the slow decay of polymer bonds in PC leading to monomer release into foods and liquids. A number of studies in various countries have 
indicated that the vast majority of the population (91-99\%) has detectable levels of BPAconjugates in their urine. The measured BPA levels reflect the recent exposure of the past several hours just before the sample collection in view of the rapid conjugation and elimination half-time of BPA in blood of a few hours. Free and conjugated BPA levels in blood are typically $\leq 1 \mu \mathrm{g} / \mathrm{L}$, which is consistent with the known rapid plasma clearance of BPA and its metabolites and kinetic studies conducted in humans.

Notably, regarding BPA determination, the analytical method used to detect both the parent compound and its metabolites is crucial especially at the low levels expected in biological samples. Therefore, the sampling and analytical methods used represent a relevant source of differences among available studies. A potential artifact in BPA measurements is the leakage of BPA from the labware used, which results in sample contamination. Indeed, as BPA has a widespread use (e.g. blood sampling devices) contamination can occur, which is particularly relevant for plasma samples and can lead to erroneously elevated concentrations of BPA in the samples.

In urine, BPA is present mainly in its conjugated form. Urinary biomonitoring data provide information on the internal dose, which is the result of total BPA exposure, independently from the sources. Therefore, biomonitoring data in urine account not only for dietary exposure, but also for non-food sources (e.g. medical devices, dust, thermal and other kinds of papers). Since BPA urinary excretion is almost complete within 24 hours after exposure and due to the less invasive sampling, urine is the matrix of choice for assessing daily exposure to BPA in humans. Urine BPA levels depend on frequency of exposure (e.g. food intake, treatment with medical devices), time of sampling after exposure, the last urination and urine production rate. Blood and urine concentrations of total BPA (free plus conjugates) determined at one time point are not representative of an average exposure, because it is strongly dependent on the time of blood sampling with respect to the exposure time.

On the basis of available biomonitoring and exposure data, it was concluded that the exposure to BPA from non-food sources that some authors hypothesized as being potentially relevant sources, is generally lower than that from exposure from food by at least one order of magnitude for most general population subgroups that were studied. Dietary exposure was indeed estimated to contribute more than $90 \%$ to the overall BPAexposure for non-occupationally exposed individuals. However, few data are available for patients treated with BPA-containing medical devices.

More recently EFSA (2015) estimated the dietary exposure to be highest for infants and toddlers among the population older than 6 months, with an estimated average of 375 $\mathrm{ng} / \mathrm{kg} \mathrm{b.w./day} \mathrm{and} \mathrm{a} \mathrm{highest} \mathrm{estimated} \mathrm{dietary} \mathrm{exposure} \mathrm{of} 857 \mathrm{ng} / \mathrm{kg} \mathrm{b.w./day.} \mathrm{The}$ modeled dietary exposure for teenagers, adults and the elderly ranged from 116 to 159 $\mathrm{ng} / \mathrm{kg}$ body weight per day for average exposure, with a high exposure ranging from 341 to $388 \mathrm{ng} / \mathrm{kg}$ body weight per day. The non-dietary exposure to thermal paper, when expressed as an oral dose equivalent, was estimated for adolescents and adults to be two to three times higher than the oral exposure (EFSA 2015).

For risk assessment purposes, the bioavailability of free BPA is crucial as this is the active compound. However, data on both free and conjugated BPA are required to assess the exposure and fate of BPA. However, regarding BPA exposure measurements there may be potential and sometimes unknown sources of BPA contamination of samples that make measurements of the non-conjugated (aglycone) BPA in blood difficult to interpret. This also may apply to studies with low dose BPA effects that did not include measurements of internal BPA exposures.

\section{Exposure from medical devices}

Medical devices based on PC and polysulfone due to their chemistry can contain BPA residues, whereas others like PVC-based medical devices may or may not contain BPA residues depending on their production method. In addition, some other BPA-derivatives (such as epoxy resins) are used specifically in dental materials. The major factor influencing the residual amount of BPA levels is the employment of incorrect operating 
conditions during the processing step. Moreover, breakdown or hydrolysis of the PC polymer after manufacturing can occur, thus giving rise to the free monomer from the polymer available for exposure. In PC articles used for food contact, the residual content is usually less than $10 \mu \mathrm{g} / \mathrm{g}$ of PC (ECB, 2003).

Exposure can be estimated by either measuring the BPA content of the medical devices or by extraction assays for potential release. Extraction of BPA was much more prominent in aqueous ethanol $(17.2 \% \mathrm{v} / \mathrm{v})$ and bovine serum (mimicking human serum) than in water. For PC casings of hemodialyzers and hollow fibers used in hemodialyzers, extracted amounts of BPA were reported up to $12.2 \mathrm{mg} / \mathrm{kg}$ material. Under simulated use conditions, release in bovine serum was up to $2090 \mathrm{ng} /$ dialyzer, and in aqueous ethanol $(17.2 \% \mathrm{v} / \mathrm{v})$ up to $4300 \mathrm{ng} /$ dialyzer. For dental materials, the leakage is limited to resins composed of Bis-DMA (Bisphenol A dimethylacrylate) which has an ester linkage that can be hydrolysed to BPA, whereas the ether linkage in Bis-GMA (Bisphenol A glycidyl methacrylate) was found to be stable. Dental materials release BPA especially shortly (0$1 \mathrm{~h}$ ) after placement: the levels of BPA detected in saliva ranged from $0.64 \mathrm{ng} / \mathrm{mL}$ to 30 $\mu \mathrm{g} / \mathrm{mL}$. This wide range in BPA measurements may indicate a continuous reduced leaching of BPA from dental materials, as well as reflect variations in the analytical methodology used.

For BPA exposure resulting from the use of medical devices, little information is available.

For the placing of dental composite resin restorations, measurements have shown that the release of BPA mainly occurs during the few hours directly after application while the BPA level is back to pretreatment levels at 24 hours. Values measured were up to 30 $\mu \mathrm{g} / \mathrm{mL}$ saliva, and $931 \mu \mathrm{g}$ in total saliva volume produced in one hour. Calculations based on the actual amount of material used in clinical practice and a median 4-year life-time of a composite restoration, suggest a maximum exposure of $0.06 \mu \mathrm{g} \mathrm{BPA} /$ day from fissure sealants, and a maximum exposure of $0.36 \mu \mathrm{g}$ BPA/day from composite restorations. Contact with dental materials gave an estimated acute $(<24 \mathrm{~h})$ exposure of 140 to 200 $\mathrm{ng} / \mathrm{kg}$ body weight per day for children and adults, respectively, whereas long term exposure ranges from 2 to $12 \mathrm{ng} / \mathrm{kg} \mathrm{b.w./day.} \mathrm{These} \mathrm{BPA} \mathrm{releases} \mathrm{contribute} \mathrm{to} \mathrm{the} \mathrm{oral}$ exposure of BPA. In general, there was very limited information provided to assess the reliability of available data.

Taking into account the many possible sources of exposure of patients during hospital care and the scarcity of information related to release of BPA from medical devices, six critical exposure scenarios were evaluated to estimate potential exposure to BPA from medical devices taking into account the material used, information related to BPA leaching, the duration of a single treatment and the frequency of treatments, giving rise to toxicologically relevant acute, short and long term exposure. Some of the estimated BPA exposures due to medical devices are in the same range as exposure to BPA via food (high exposure for population older than 6 months $857 \mathrm{ng} / \mathrm{kg}$ b.w./day, high exposure for infants breastfed day 1-5 after birth $435 \mathrm{ng} / \mathrm{kg}$ b.w/day) (EFSA 2015). However, exposure associated with medical devices use generally occurs for a limited period of time with the exception of haemodialysis practices and the bioavailability of BPA through routes of exposure other than the oral is much higher.

Measurements in dialysis patients found total BPA values up to $6.6 \mathrm{ng} / \mathrm{mL}$ blood. In prematurely born infants undergoing intensive therapeutic medical interventions, $a$ geometric mean urinary concentration for BPA of $30.3 \mu \mathrm{g} / \mathrm{L}(\mathrm{ng} / \mathrm{mL})$ was observed with the highest value measured $946 \mu \mathrm{g} / \mathrm{L}(\mathrm{ng} / \mathrm{mL})$, which was about ten times higher than that among children 6-11 years old. More than $90 \%$ of the BPA detected in the urine of the prematurely born infants was in its conjugated (e.g. glucuronide, sulphate) form.

The highest exposures estimated occurred during i) acute contact with dental materials (140 to $200 \mathrm{ng} / \mathrm{kg}$ body weight per day for infants and adults, respectively) ii) prolonged medical procedures (such as extracorporeal circulation or transplantation/ implantation of an artificial organ) in infants ( $685 \mathrm{ng} / \mathrm{kg}$ body weight per day for a b.w. around $5 \mathrm{~kg}$ ); iii) 
treatment of adults with medical devices consisting of BPA containing PVC $(1000 \mathrm{ng} / \mathrm{kg}$ b.w./day), iv) treatment of prematurely born infants in neonatal intensive care units (NICU) (3000 ng/kg b.w./day), and v) treatment of prematurely born infants with medical devices consisting of BPA containing PVC $(7000 \mathrm{ng} / \mathrm{kg}$ body weight per day, 7 $\mu \mathrm{g} / \mathrm{kg}$ body weight per day). Exposure via medical devices consisting of BPA containing PVC might even be higher (adults up to $5000 \mathrm{ng} / \mathrm{kg} \mathrm{b.w./day,} \mathrm{infants} \mathrm{up} \mathrm{to} 12000 \mathrm{ng} / \mathrm{kg}$ b.w./day). However, it is worth noting that exposure to BPA via BPA-containing PVC has been estimated based on extrapolation from data on phthalate leakage from PVC and are, therefore, affected by a high degree of uncertainties. In addition European PVC manufacturers informed the SCENIHR that they do no longer use BPA in PVC production. However, medical devices from Third Countries, for which there is no available information, can be marketed, including gloves.

\section{BPA metabolism and toxicokinetics in humans}

The major BPA metabolite in humans is BPA-glucuronide, which is quantified in plasma and rapidly excreted in the urine; BPA-sulphate has also been detected after oral exposure as a minor metabolite. After oral exposure, there is a very high first pass effect in the liver that results in very small amounts of unchanged parent BPA. In humans, a polymorphism exists for the conjugation of BPA. However, the polymorphism was found to result in a 4-5-fold variability of plasma BPA. Therefore, it can be considered that the default value used in the risk assessment to account for kinetic interindividual variability within the general population can cover the differences due to polymorphically expressed enzyme activity involved in BPA metabolism.

Studies on toxicokinetics of BPA available to date have demonstrated a significantly lower internal exposure to free BPA after oral intake as compared to parenteral exposure. This is essentially due to the highly efficient pre-systemic conjugation to glucuronide and sulphate, which occurs mainly in the liver and partially in the gut after oral administration independently on the species. Thus, the internal exposure to free BPA after oral intake is lower as compared to dermal or parenteral exposure, although for the latter routes of exposure, the biotransformation (mainly in the liver) quickly diminishes free circulating BPA.

After oral exposure, both low and high single oral doses of BPA are well absorbed $(>90 \%)$, but the systemic bioavailability of free BPA after oral exposure is reduced by the first pass effect. Based on the analysis of oral versus intravenous toxicokinetic data (Doerge et al., 2010a, 2010b; 2011; 2012), the oral systemic bioavailability of unconjugated BPA is $2.8 \%, 0.2 \%, 0.9 \%$ and less than $1 \%$ in rats, mice, monkeys, and dogs, respectively. The systemic availability of unconjugated BPA in humans has not been evaluated experimentally, however, modelled data as well as controlled biomonitoring studies indicated that internal exposure in humans to unconjugated BPA is very low (1-10\%) (Mielke and Gundert-Remy, 2012; ANSES, 2013). The conjugates are readily excreted in urine; as a consequence the half-life of BPA in humans is very short, ranging from 1 to $3.5 \mathrm{~h}$. After dermal exposure, the absorption fraction can be considered approximately $25-30 \%$ of the applied dose, which is directly systemically bioavailable.

For all the parenteral routes of exposure (including intravenous, intraperitoneal, transdermal or subcutaneous), the chemical is $100 \%$ systemically bioavailable: however, the clearance of free BPA from the circulation appeared to be quite fast, as indicated by controlled studies in non- human primates and rats with $>50 \%$ of circulating BPA already conjugated $5 \mathrm{~min}$ after intravenous injection, and showing a half-life of $0.66 \mathrm{~h}$ in rats.

The available modeled data, obtained after oral exposure, indicate that newborns and babies up to 6 months constitute a potentially susceptible subpopulation due to potential immature BPA metabolism. However, the default uncertainty factor which is used to account for the toxicokinetic variability in the general population seems to be large enough to cover the variability in the newborn population exposed via the oral route. 


\section{Pharmacokinetics in animals}

Rats show a prolonged clearance of BPA due to the existence of the so-called enterohepatic recirculation. After uptake from the GI-tract in rats there is a high degree of conjugation of BPA in the liver. However, BPA is excreted from the liver via bile into the GI-tract where it can be cleaved again resulting in free BPA that can be recirculated or excreted via the faeces. Thus, there may be a higher exposure to free BPA when compared with humans especially in neonatal animals after a specific oral dose. It may be considered that neonatal effects studies in mice and rats may over-predict adverse outcomes in humans. A general problem in determining BPA (free or conjugated) levels in serum is the possible contamination with BPA from unknown sources, in the serum samples due to collection, storage and analysis. This last issue has been demonstrated to also occur in human serum samples.

\section{Toxicity of BPA}

Several toxicity studies have been performed in rodents and dogs. BPA was found to be of low acute toxicity for all routes of exposure relevant to human health. The lowest NOAEL after oral repeated exposure was indicated in the previous evaluations as approximately $5 \mathrm{mg} / \mathrm{kg}$ b.w./day, based on effects on the liver as target organ, as identified in several studies, including multigeneration reproductive toxicity studies. The next lowest NOAEL for oral exposure was $50 \mathrm{mg} / \mathrm{kg} \mathrm{b.w./day,} \mathrm{based} \mathrm{on} \mathrm{toxic} \mathrm{effects} \mathrm{on}$ the kidney and reproductive toxicity.

By applying the benchmark dose (BMD) approach, EFSA recently calculated a $\mathrm{BMDL}_{10}$ of $8.96 \mathrm{mg} / \mathrm{kg}$ b.w./day using data from the same multigeneration reproductive toxicity study in mice from which the NOAEL of $5 \mathrm{mg} / \mathrm{kg} \mathrm{b.w./day} \mathrm{was} \mathrm{previously} \mathrm{derived} \mathrm{(EFSA}$ 2015). The $\mathrm{BMDL}_{10}$ represents the lower level of the confidence interval of the effect resulting in a $10 \%$ deviation from vehicle-treated control animals. The critical endpoint (i.e. appearing at the lowest dose) for this $\mathrm{BMDL}_{10}$ was the alteration in kidney weight.

BPA is not a mutagen in in vitro test systems, nor does it induce cell transformation. BPA has been shown to affect chromosomal structure in dividing cells in in vitro studies, but evidence for this effect in in vivo studies is inconsistent and inconclusive. In addition, BPA was found to be genotoxic in in vitro micronucleus assays. These findings were not confirmed by in vivo studies. Therefore, BPA is not likely to pose a genotoxic hazard to humans.

In standard carcinogenic OECD testing protocols, BPA has no carcinogenic activity. In addition, in multigeneration studies, no indication of increased cancerogenicity was observed. Studies using subcutaneous administration of BPA suggested that BPA may have the ability to increase the effects of well-known carcinogens even at very low BPA levels. However, these studies had limitations which render them unsuitable to assess whether BPA has a carcinogenic potential (as initiator or promoter) by prenatal or perinatal exposure.

Prenatal exposure to BPA by subcutaneous injection in pregnant rats induced mammary gland alterations in the offspring including cell proliferation, some described as preneoplastic and neoplastic lesions. Similar results were obtained in mouse studies and results observed in rhesus monkeys also indicated alterations of glandular tissue in the mammary gland after prenatal exposure. Also, in a recently published animal study there were indications for mammary gland ductal proliferation (Delclos et al., 2014).

In summary, at present there are no indications for carcinogenic effects of BPA in OECD guideline studies, but there are some effects observed in the mammary gland. The observed proliferative effects on mammary gland raise some concern and has been included in the risk assessment recently performed by EFSA. However, the biological significance of such alterations for humans is currently unknown.

The interpretation of neurotoxic and neurobehavioural effects of BPA is uncertain. Studies on anxiety (rodent and non-human primate) have a behavioural end-point which is highly dependent on study design, testing apparatus, inclusion of only one sex, and age at 
examination. In several studies, increased anxiety was observed. However, there is uncertainty with regard to the interpretation of the data. Recent data indicate sexdimorphic effects of BPA on social behaviour. However, it is uncertain whether such an effect could be considered adverse for humans. Additionally, gene expression in the brain was altered after both prenatal BPA exposure and BPA exposure in adult mice. Other effects described in the recent studies may indicate that the effects observed with BPA on hypothalamic organization involves mechanisms different from its estrogenic action because they are very different from those of oestradiol which was used as positive control. The variety of read-out parameters and the effects observed warrant further investigation of the possible neurological and behavioural effects of BPA, as well as their relevance for humans, displaying a different pattern of brain development when compared to rodents. However, this effect was included in the uncertainty analysis by EFSA (2015).

BPA is able to elicit skin sensitization in humans, probably because it is a weak sensitizer. Studies on a possible relationship between prenatal and/or postnatal BPA exposure and allergic responses are not consistent. Although effects on the immune system are suggested, there is uncertainty on the immunotoxicity of BPA. In view of the suggested effects of BPA on the immune system, further investigation to determine potential immunotoxicity of BPA is warranted. However, this effect was included in the uncertainty analysis by EFSA (2015).

The toxicological data do not indicate a clear effect of BPA on cardiovascular function.

Several published studies have directly addressed the issue of whether developmental exposure to BPA can affect adiposity, glucose or insulin regulation, lipid profiles or other end-points related to diabetes or metabolic syndrome. Animal studies, however, have shown an increase, a decrease and no effect on body weight. The discrepancy among the various animal studies may arise from variation in experimental conditions, such as the dosing regimen, animal species and strains, and the timing of evaluation of effects. A number of studies in prenatally- and postnatally- exposed rats and mice suggest that BPA exposure has an effect on metabolic function.In some of the studies the findings have been claimed as evidence of a non-monotonic dose-response, as effects seen at a lower dose were not observed at higher doses. However, these effects were only seen at one dose level. There are no studies which demonstrate effects of different effect size at two dose levels and no or a reduced effect at a higher dose, thus corroborating the existence of non-monotonicity. There is, however, no convincing evidence that BPA is obesogenic later in life after intrauterine exposure or in longer-term studies.Thus, regarding a metabolic effect of BPA. no clear conclusions can be drawn at present due to a lack of consistent evidence. Additionally, in epidemiological studies, inconsistent results were obtained. Therefore, this issue still warrants further investigation. However, this effect was included in the uncertainty analysis by EFSA (2015).

A large number of studies is available on the effects of BPA on reproduction and prenatal development, some of which performed according to internationally agreed guidelines and compliant to GLP principles. A wealth of in vitro results and studies on non-intact animals (such as ovariectomized rodents) is available, but their value for risk assessment is questionable. There are also uncertainties as to reproducibility of several individual studies. These studies were conducted in rats and mice. Female reproductive toxicity after oral exposure occurred with an overall NOAEL of $50 \mathrm{mg} / \mathrm{kg}$ b.w./day and a LOAEL of $500 \mathrm{mg} / \mathrm{kg}$ b.w./day. However, at the LOAEL for female reproductive effects, significant body (or organ) weight reduction and hepatic/renaltoxicity occurred (i.e. the effects used as Point of Departure for the TDI derivation). As to developmental toxicity, BPA does not cause malformations or birth defects in rats or mice at levels up to the highest doses evaluated: $640 \mathrm{mg} / \mathrm{kg} /$ day (rats) and $1250 \mathrm{mg} / \mathrm{kg} /$ day (mice). Therefore, it can be concluded that reproductive or developmental toxicity are not the critical end-points in BPA toxicity, although it does have reproductive toxicity at doses higher than those causing liver and kidney damage. On this base BPA was classified as a Repro 1B toxicant (ECHA, 2014). 
In conclusion, there are indications that BPA may have biological effects below the $\mathrm{BMDL}_{10}$ of $8.96 \mathrm{mg} / \mathrm{kg}$ b.w./day (oral exposure). However, the evidence has not been obtained in general toxicity studies, but mainly in dedicated studies focussing on specific outcome parameters like adiposity and hormone levels. The effect on some of those parameters resulted in contrasting results in various studies (e.g. decrease, no effect or increase in body weight). In addition, dose-response relationships could not be established. Regarding possible low dose effects, some concern remains for effects on mammary gland, metabolism and adiposity and neurobehavior Further extensive doseresponse and/or epidemiological studies are needed to confirm or negate these observations and their relevance for human health effects.

In its recent evaluation EFSA (2015) used for the derivation of the t-TDI a Benchmark dose modelling approach and derived a $\mathrm{BMDL}_{10}$ value of $8960 \mu \mathrm{g} / \mathrm{kg} \mathrm{b.w./day} \mathrm{based} \mathrm{on}$ effects on the kidney in a general toxicity study in mice. The $B M D L_{10}$ dose was translated into a human dose inducing similar effects, the human equivalent dose (HED) $=609$ $\mu \mathrm{g} / \mathrm{kg}$ b.w./day, determined considering the ratio of internal exposure in mice versus the internal exposure in humans based on toxicokinetic studies. An assessment factor of 25 could be established based on the following considerations: 1 (as the HED is accounting for interspecies differences in kinetics) $\times 2.5$ (interspecies dynamic factor) $\times 10$ (intraspecies factor) $=25$. An additional uncertainty factor of 6 was applied as a result of the uncertainty analysis related to mammary gland, reproductive, neurobehavioural, immune and metabolic system effects. So, a BPA specific total uncertainty factor of 150 was applied resulting in the t-TDI of $4 \mu \mathrm{g} / \mathrm{kg} \mathrm{b.w./day.} \mathrm{In} \mathrm{view} \mathrm{of} \mathrm{the} \mathrm{still} \mathrm{present}$ uncertainties the TDI was declared temporary.

The SCENIHR considers that the recently derived temporary oral TDI ( $t$-TDI) of $4 \mu \mathrm{g} / \mathrm{kg}$ b.w./day by EFSA which takes overall all effects and uncertainties into account (as well as the use of a BPA specific assessment factor of 150), represents a useful base for carrying out a BPA risk assessment for the use of BPA in medical devices.

\section{Epidemiological studies}

There are a limited but increasing number of epidemiological studies that investigated an association between BPA exposure and health outcomes. Studies have also been performed to determine potential altered behavior after dental BPA exposure. Epidemiological studies regarding effects of BPA on metabolic disorders and/or obesity show inconsistent results. Most of them use cross-sectional designs which are not suitable for establishing a cause-effect relationship, especially for outcomes that have long latency periods (e.g. cardiovascular disease, diabetes). Many of these crosssectional analyses have several important weaknesses that limit their interpretation and relevance. For instance, a major limitation is the use of a single spot urine sample that reflects recent BPA exposure only (past several hours) and may not adequately measure BPA exposure during the relevant etiological window for health outcomes like cardiovascular disease and diabetes, which might be years or decades earlier.

For further clarification and interpretation of the relationship between BPA exposure and adverse health effects, additional studies are needed. These should include prospective studies with serial exposures to BPA assessed during etiologically relevant windows, years before development of disease, and thus prolonged follow up periods.

\section{Conclusions on health effects}

Regarding potential health effects of BPA exposure and the level of exposure, several controversial issues remain, regarding the possibility that BPA has biological effects below the $\mathrm{BMDL}_{10}$ of $8.96 \mathrm{mg} / \mathrm{kg}$ b.w./day (oral repeated exposure), such as prenatal BPA exposure and an effect on mammary gland development and altered behaviour/anxiety, in addition to possible effects on metabolism and adiposity. However, in a recent report from studies in rats including both a low dose range and two high doses, toxicity (including effects on the mammary gland) was only observed in the high dose groups whereas doses below the NOAEL of $5 \mathrm{mg} / \mathrm{kg} \mathrm{b.w./day} \mathrm{(oral} \mathrm{exposure)} \mathrm{did}$ not induce toxicity (Delclos et al., 2014). To date, available epidemiological studies 
performed do not provide consistent outcomes to conclude on possible human health effects. These aspects need further investigation in properly conducted studies with a wide dose range of BPA.

\section{Alternatives for BPA}

Several alternatives for BPA exist and are increasingly used, notably Bisphenol S and Bisphenol $F$ and some halogenated bisphenol $A$ derivatives (e.g. tetrachlorobisphenol $A$ and tetrabromobisphenol A). For some of the alternatives, similar effects as for BPA were reported regarding endocrine activity in in vitro assays, although with reduced activity/potency when compared to BPA. The general toxicological profile of alternatives is much less known.

\section{Recommendations for research}

The risk of BPA exposure from medical devices is associated with the release of BPA from these devices, although the actual level of leaching in the different conditions and the consequent level of exposure is poorly characterised and deserves further investigation. For sterilization of medical devices, it is known that steam sterilization may result in release of BPA from PC medical devices. Whether ethylene oxide (EtO) sterilization induces release of BPA from PC or PSU medical devices is yet unknown. Therefore, research into the use and consequences of EtO sterilization with regard to BPA release is also recommended.

Regarding possible low dose effects, some concern remains for effects on mammary gland, immune system, metabolism and adiposity and neurobehavior. Further extensive dose-response studies (including a broad range of BPA doses) are needed to confirm or negate these observations and their relevance for human health effects.

Human studies should include prospective epidemiological studies with serial exposures to BPA assessed during etiologically relevant windows, years before development of disease, and prolonged follow up periods.

\section{Risk assessment associated with BPA-containing medical devices}

For medical devices, several exposure scenarios were identified taking into account the material used, information related to BPA leaching, the duration of a single treatment and the frequency of treatments. They included external short-term contact with a medical device, short- and long-term contact with dental materials, short- and long-term contact with an implanted medical device, long term contact via hemodialyzers and contact in intensive care units with various medical devices. They were evaluated in view of toxicologically relevant acute, short- and long-term exposure.

Exposure due to medical devices generally occurs for a limited period of time, with the exception of haemodialysis patients. Indeed, for implanted medical devices, the release of BPA is higher after the implantation and rapidly decreases with time to zero levels (kinetic being determined by the BPA initial content).

For BPA exposure resulting from the use of medical devices, little information is available and in many cases due to the lack of experimental data, estimates were used. The uncertainties related to the exposure assessment represent indeed the weakest part of this evaluation. For this reason only the highest value obtained in the estimate was indicatively used.

The estimated highest BPA exposures for different scenarios were: 1) $3000 \mathrm{ng} / \mathrm{kg}$ b.w./day (3 $\mu \mathrm{g} / \mathrm{kg}$ b.w./day) for prematurely born infants in neonatal intensive care units; 2) $685 \mathrm{ng} / \mathrm{kg}$ b.w./day for prolonged medical procedures (such as extracorporeal circulation or transplantation/ implantation of an artificial organ) in infants (b.w. around $5 \mathrm{~kg}$ ); 3) $57 \mathrm{ng} / \mathrm{kg}$ b.w./day for dialysis patients; 4) $0.4-12 \mathrm{ng} / \mathrm{kg} \mathrm{b.w./day} \mathrm{for} \mathrm{long-}$ term exposures to medical devices; 5) 140 to $200 \mathrm{ng} / \mathrm{kg} \mathrm{b.w./day} \mathrm{for,} \mathrm{respectively,}$ children and adults due to contact with dental materials $(<24 \mathrm{~h}) ; 6) 2$ to $12 \mathrm{ng} / \mathrm{kg}$ 
b.w./day due to long-term contact with dental materials, far below the exposure to BPA via food (EFSA 2013, EFSA 2015).

The exposure of neonates is about 13-fold higher than the mean dietary exposure of breastfed infants at days 1-5 after birth ( 7 fold higher than the high exposure for infants day 1-5 after birth $435 \mathrm{ng} / \mathrm{kg}$ b.w/day), whereas the external dose related to infants repeated treatments with medical devices was in the same range (high exposure for population older than 6 months $857 \mathrm{ng} / \mathrm{kg}$ b.w./day) (EFSA 2013, EFSA 2015). However, considering the different bioavailability of the two routes (oral vs parenteral) the internal exposure due to medical devices is 2-3 order of magnitude higher.

Exposure via medical devices consisting of BPA containing PVC might even be higher (adults up to $5000 \mathrm{ng} / \mathrm{kg}$ b.w./day, infants up to $12000 \mathrm{ng} / \mathrm{kg} \mathrm{b.w./day).} \mathrm{However,}$ exposure to BPA via BPA-containing PVC has been estimated based on extrapolation from data on phthalate leakage from PVC with an extremely high degree of uncertainties. In addition European PVC manufacturers informed that they do no longer use BPA in their PVC production, Hence, it is unlikely that such a high BPA exposure will be reached due to the use of medical devices consisting of BPA containing PVC produced in Europe. However, medical devices from Extra-EU countries can be marketed including gloves, on which no information is available.

The oral long-term exposure via dental material is far below the recently determined oral t-TDI of $4 \mu \mathrm{g} / \mathrm{kg}$ b.w./day (EFSA 2015) and pose negligible risk for human health.

Regarding the parenteral exposure via medical devices, it is necessary to consider the differences in bioavailability (assumed as $100 \%$ ) when compared to the oral route (around $1 \%$ ). Therefore, the SCENIHR considered it appropriate to make the comparison using the estimated internal dose $(6 \mu \mathrm{g} / \mathrm{kg} \mathrm{b.w./day}=\mathrm{HED} / 100)$ rather than the external t-TDI.

Taking treatment of neonates in intensive care units as an example, exposure of $3 \mu \mathrm{g} / \mathrm{kg}$ b.w./day (assuming as $100 \%$ systemically bioavailable) is, before adjustment for route specific systemic availability, below the oral t-TDI of $4 \mu \mathrm{g} / \mathrm{kg} \mathrm{b.w./day.} \mathrm{However,} \mathrm{it} \mathrm{is}$ about 75-fold higher when compared to the internal exposure to free BPA using the oral t-TDI (being $0.04 \mu \mathrm{g} / \mathrm{kg}$ b.w./day based on a t-TDI of $4 \mu \mathrm{g} / \mathrm{kg} \mathrm{b.w./day} \mathrm{with} 1 \%$ systemic bioavailability for the oral dose). Alternatively, applying a MOS approach, when the internal HED of $6 \mu \mathrm{g} / \mathrm{kg} \mathrm{b.w./day} \mathrm{was} \mathrm{compared} \mathrm{to} \mathrm{the} \mathrm{internal} \mathrm{exposure} \mathrm{via} \mathrm{medical}$ devices $(3 \mu \mathrm{g} / \mathrm{kg}$ b.w./day) a MOS= 2 was obtained, much lower than the assessment factor of 150 used for the t-TDI derivation.

For the other scenarios the MOS is 10 for repeated medical procedures in infants; for the other short-term exposure scenarios estimated for different medical devices, the MOS ranges between 43 and 100; for dialysis treatments the MOS is 105 . For the other scenarios the exposure ranges from $0.4 \mathrm{ng} / \mathrm{kg} \mathrm{b.w./day} \mathrm{to} 12 \mathrm{ng} / \mathrm{kg} \mathrm{b.w./day} \mathrm{resulting} \mathrm{in}$ a MOS range from 500 to 15000 .

In relation to exposure to BPA released from dental materials during the first 24 hours after placement, uptake in the oral cavity by oral mucosa can be considerable. Assuming $100 \%$ as a worst case scenario, for the highest exposures $200 \mathrm{ng} / \mathrm{kg} \mathrm{b} . \mathrm{w} . /$ day the MOS would be 30 . However, the assumption of $100 \%$ bioavailability is largely exaggerated and the peak of exposure occurs for $<24 \mathrm{~h}$ (representative of acute exposure in a toxicological context) whereas the MOS is related to chronic exposure. The differences in exposure duration can be larger than 5, therefore a MOS of 30 for acute exposure to dental materials can be considered sufficiently large.

Duration of exposure is indeed very relevant. With the exception of dialysis patients for which treatment is repeated for long time periods and can be considered similar to chronic exposure, the exposure via medical devices is generally of limited duration 
(representative of acute to subchronic exposure in a toxicological context), whereas the MOS is based on long-term exposure. The application of correction factors to the MOS accounting for the time of exposure ( 3 as used by ECHA from sub-chronic to chronic) might indicate that MOS around 50 could be sufficiently large, although with a high degree of uncertainties. Nevertheless some scenarios still remains in the area of concern.

The SCENIHR concludes that risk for adverse effects of BPA may exist when the BPA is directly available for systemic exposure after non-oral exposure routes, especially for neonates in intensive care units, infants undergoing prolonged medical procedures and for dialysis patients. Although the benefit of medical devices must also be considered, the SCENIHR recommends that where practicable, medical devices that do not leach BPA should be used. The possibility of replacing BPA in these products should be considered against their efficiency in the treatment, as well as the toxicological profile of the alternative materials.

However, the present risk assessment may be refined when new data on exposure via medical devices becomes available. 


\section{BACKGROUND}

Bisphenol A (BPA) is an intermediate that is mainly used in combination with other chemicals to manufacture plastics and resins. For example, BPA is used in polycarbonate, a high performance transparent, rigid plastic used to make food containers, such as returnable beverage bottles, tableware (plates and mugs) and storage containers. Residues of BPA are also present in epoxy resins used to make protective coatings and linings for food and beverage cans and vats. BPA can migrate in small amounts into food and beverages stored in materials containing the substance.

BPA is a weak oestrogen, as demonstrated by in vitro studies. Many in vivo studies have been performed to examine its potential effects on reproduction and development. The safety of BPA in food contact materials has been evaluated by the US Food and Drug Administration (FDA) ${ }^{1}$ and by the European Food Safety Authority (EFSA) ${ }^{2}$. Although these evaluations did not identify reasons for concern, a number of uncertainties in the current scientific knowledge concerning the safe use of BPA remain. Considering these uncertainties, especially with respect to potential adverse health effects of BPA exposure to infants through polycarbonate baby bottles, the European Commission decided on the basis of the precautionary principle that all baby bottles on the EU market containing BPA should be replaced by the middle of 2011.

Recently, safety concerns were expressed for vulnerable groups such as infants, pregnant and breast-feeding women exposed to BPA through other products. Medical devices are a particular product category in which BPA is often found. Examples include implants, catheters, and dental devices fabricated from certain materials (e.g. polycarbonate or bisGMA) that may contain and release residual BPA present in these devices. Some BPA-containing medical devices may have direct and/or indirect contact with the patients (e.g. autotransfusion apparatus, filters, bypasses, tubing, pumps, instruments, surgical equipment, blood pathway circuits and respiratory tubing circuits). These products are used in all types of patients of all ages.

Due to the common use of polycarbonate plastic and epoxy resins in such a wide range of products, low-level human exposure to BPA occurs, but the health significance of the exposure levels has been controversial. According to Council Directive 93/42/EEC, medical devices may only be placed on the market if they meet the essential requirements laid down in its Annex I. The devices must be designed and manufactured to be used under the conditions and for the purposes intended. Moreover, they must not compromise the clinical condition or the safety of patients, or the safety and health of users or, where applicable, other persons, provided that any risks which may be associated with their use constitute acceptable risks when weighed against the benefits to the patient.

\footnotetext{
${ }^{1}$ http://www.fda.gov/newsevents/publichealthfocus/ucm064437.htm

${ }^{2}$ http://www.efsa.europa.eu/en/topics/topic/bisphenol.htm
} 


\section{TERMS OF REFERENCE}

The Scientific Committee on Emerging and Newly Identified Health Risks (SCENIHR) is requested to provide a scientific Opinion on 'The safety of the use of bisphenol $A$ in medical devices'.

In particular, the SCENIHR is asked:

1. To determine whether levels of exposure to BPA from the use of the various medical devices containing BPA could raise health concerns and, if possible, to provide indications on limit values for BPA release from medical devices.

2. To identify whether any particular medical devices containing BPA could result in human exposures which would raise concern under their normal use patterns or other foreseeable circumstances (e.g. high release of BPA due to the nature of the material of the medical device or to particular contact conditions).

3. To identify any patient group, e.g. infants, pregnant and breastfeeding women, who would be particularly at risk in light of the questions raised here.

4. In case reasons for concern related to BPA are identified, to propose possible alternative approaches that could reduce potential risks either by identifying alternative practices or by identifying alternatives to the use of BPA in medical devices. If no clear answer can be provided on this point, the SCENIHR is asked to formulate recommendations for research that could help provide scientific evidence to that end. 


\section{SCI ENTI FI C RATI ONALE}

\subsection{Introduction}

Bisphenol A [Bis(4-hydroxyphenyl)propane], BPA is a high-production volume industrial chemical. About 3.8 million tons BPA were produced worldwide in 2006 (Plastic Europe 2007, WHO/FAO 2010). More than 95\% of the BPA produced is used to manufacture polycarbonate (PC) plastic and as a precursor of the manufacturing of monomers of epoxy resins (Plastic Europe 2007, WHO/FAO 2010, Beronius and Hanberg 2011, Genuis et al., 2011). BPA is also used in the production of the flame retardant tetrabromobisphenol $A$, in the production of thermal paper, as an antioxidant in plasticizers and for inhibiting the polymerization in polyvinyl chloride (PVC). The European Council of Vinyl Manufacturers stated that the use of BPA for polymerization and stabilisator for storage of vinyl chloride in Europe was discontinued as of December 2001 (KEMI 2011). However, PVC as a source of BPA exposure cannot be completely excluded because BPA-containing PVC may still be used in the EU due to the global market for medical devices.

BPA is a key building block of PC plastic. Polycarbonate plastic is a lightweight, highperformance plastic that possesses a balance of toughness, dimensional stability, optical clarity, high heat resistance and electrical resistance. Because of these attributes, PC is used in a wide variety of common products such as food and drink packaging materials, plastic water bottles and infant feeding bottles, digital media (e.g. CDs, DVDs), electrical and electronic equipment, construction glazing, sports safety equipment and medical devices. The durability, shatter-resistance and heat-resistance of PC make it a good choice for tableware as well as for reusable bottles and food storage containers that can be conveniently used in the refrigerator and microwave. As an alternative to PC, polysulfone (PSU) is also used in some medical devices. As polysulfones are polymers obtained by a reaction between BPA and bis(4-chlorophenylsulfone), producing an etheroxide, leaching of BPA from PSU is possible. BPA-resins (such as BADGE: Bisphenol A diglycidyl ether, (see Figure 1, page 27) are used as lacquers to coat metal products such as food cans, bottle lids, coatings inside tanks used for drinking water and waste water, large wine storage tanks and water supply pipes.

In addition to PC medical devices, some dental materials are fabricated from monomers such as bisphenol A glycidyl methacrylate (Bis-GMA, Figure 1) and very few from bisphenol A dimethacrylate (Bis-DMA, Figure 1) derived from BPA (Fleisch et al., 2010). BPA-resins are also used in inks and adhesives. Polymers produced using BPA (e.g.polysulfone) are used as membranes in hemolysis dialysers. A detailed description of uses for BPA, PC, and epoxy resins is listed elsewhere (Beronius and Hanberg, 2011). Trace amounts of BPA are present, as residues of polymerization process, in PC, epoxy resins and dental sealants.

About $3 \%$ of total PC production is used for the manufacture of medical devices (Beronius and Hanberg, 2011). Various medical devices produced with BPA derived materials (PC, polysulfone and BPA-resins) are mentioned in Annex 1.

Several health risk assessments of BPA have been conducted by regulatory authorities as well as expert groups based on oral exposure (ECB, 2003, EC2010b; EFSA 2006 and 2010, NTP-CERHR 2008, Environment Canada/Health Canada 2008, WHO/FAO 2010; USFDA, 2013). ANSES (2011) provided a report on the hazard identification of BPA. The risk assessment methodology used in this Opinion includes the conclusions of the earlier risk assessments updated with recent relevant data on BPA.

Other bisphenols [for example Bisphenol B:Bis(4-hydroxypheny)butane, BPE: Bis(4hydroxyphenyl)ethane], Bisphenol F: Bis(4-hydroxyphenyl)methane, and resins derived from it (BFDGE: Bisphenol $F$ diglycide ether) and bisphenol-S [bis(4- 
hydroxyphenyl)sulfone, (BPSU)] are also used for similar purposes as BPA as well as for the resins derived from it.

Finally, halogenated derivatives of BPA, such as tetrabromobisphenol-A [2,2-bis(4hydroxy-3,5-dibromophenyl)propane, (TBBPA)] and tetrachlorobisphenol-A [2,2-bis(4hydroxy-3,5-dichlorophenyl)propane, (TCBPA)] are both widely used as flame-retardants for building material, paints, plastic products including epoxy resin, electronic circuit boards and other electronic equipment. This Opinion does not include risk assessment of these BPA derivatives and the alternative substances. However, some BPA-derivatives are considered, because they can release BPA.

This Opinion focuses on adverse effects and risk assessment of exposure to BPA via medical devices, including parenteral and oral applications. Information on adverse effects after oral exposure to BPA is based on the literature published after the existing evaluation between 2010 and 2013. For other routes of exposure, a more comprehensive overview is presented, including literature published before 2010.

\subsection{Methodology}

This Opinion includes an analysis of experimental, clinical and epidemiological evidence on adverse effects on human health arising from BPA-containing medical devices. Recent scientific evidence was reviewed to determine whether BPA-based polycarbonates, resins and/or BPA containing PVC are associated with health risks

The SCENIHR considered evidence from peer-reviewed scientific and medical literature and published reports of institutional, professional, governmental and non-governmental organisations. Unpublished work or public opinions not scientifically substantiated were not considered. Numerous peer-reviewed scientific publications on BPA were used for this Opinion.

The SCENIHR reviewed and evaluated the potential risk of the use of BPA in medical devices against clinical benefit. In a weight of evidence approach, lines of evidence or hypotheses for causality are evaluated based on supportive studies. When a line of evidence is consistently supported by various studies (i.e. evidence is independently reproduced in different studies) causality is likely between the observed effect and exposure to the substance. Relevance, strength and weaknesses of the studies evaluated were considered. The weight of evidence (SCENIHR, 2012) is categorised as follows:

Strong overall weight of evidence: Coherent evidence from human and one or more other lines of evidence (animal or mechanistic studies) in the absence of conflicting evidence from one of the other lines of evidence (no important data gaps).

Moderate overall weight of evidence: good evidence from a primary line of evidence but missing evidence from several other lines (important data gaps).

Weak overall weight of evidence: weak evidence from the primary lines of evidence (severe data gaps).

Uncertain overall weight of evidence: due to conflicting information from different lines of evidence that cannot be explained in scientific terms.

Weighing of evidence not possible. No suitable evidence available.

The evidence for the presence of a causal relationship between exposure to BPA due to the use of medical devices and adverse effects are discussed in the chapters below. In addition, the risk will be evaluated against the potential benefit of the use of the various medical devices. 


\subsection{Chemistry of BPA}

\section{I dentification of the Substance}

CAS-No: 80-05-7

EINECS No: 201-245-8

IUPAC name: 2,2-bis(4-hydroxyphenyl)propane

Molecular weight: 228.29

Molecular formula: $\mathrm{C}_{15} \mathrm{H}_{16} \mathrm{O}_{2}$

Structural formula:

Smiles notation: $\mathrm{Oc}(\operatorname{ccc}(\mathrm{c} 1) \mathrm{C}(\mathrm{c}(\operatorname{ccc}(\mathrm{O}) \mathrm{c} 2) \mathrm{c} 2)(\mathrm{C}) \mathrm{C}) \mathrm{c1}$

Synonyms:

BPA (Common abbreviation)

2,2-Bis(4-hydroxyphenyl)propane

2,2-Bis(p-hydroxyphenyl)propane

$p, p^{\prime}$-Isopropylidene-bisphenol

$\mathrm{p}, \mathrm{p}^{\prime}$-Isopropylidene-di-phenol

Phenol, 4,4'-Isopropylidene-di

Diphenylol Propane

Parabis (Trademark)

Bis (4-hydroxyphenyl) dimethyl methane

Bis (4-hydroxyphenyl)propane

Dian (Trademark)

Dimethylmethylene- $p, p^{\prime}$-di-phenol

Dimethyl Bis(p-hydroxyphenyl)methane

4,4'-Dihydroxy-2,2'-diphenyl propane

4,4'-Dihydroxydiphenyldimethyl methane

4,4'-Dihydroxydiphenyl propane

B-Di-p-Hydroxyphenyl propane

$p, p^{\prime}$-Dihydroxydiphenyldimethyl methane

$p, p^{\prime}$-Dihydroxydiphenyl propane

2,2'-(4,4'-Dihdroxydiphenyl) propane

4,4'-Dihydroxydiphenyl-2,2'-propane

2,2'-Di(4-hydroxyphenyl) propane

2,2'-Di(4-phenylol) propane

4,4'-Isopropylidene bisphenol

4,4'-(1-methylethylidene)bisphenol

\section{Purity}

The EU Risk Assessment Report of 2003 defines purity of BPA as being $99-99.8 \%$ with impurities typically including phenol $(<0.06 \%)$, other isomers of bisphenol-A $(<0.2 \%)$ 
and water ( $<0.2 \%$ ) (ECB 2003). Terasaki et al. (2004) examined four samples of industrial BPA with stated purities of $97 \%$ to $98 \%$ and a sample of laboratory grade BPA ( $99+\%$ purity) and they found 15 trace impurities. However, it is presently unknown whether such impurities are also present in medical devices or if they participate in polymerization reactions. Thus, leaching of BPA from manufactured medical devices remains unknown.

\section{Additives}

There are no stated additives used with BPA.

\subsection{Physico-Chemical Properties}

The physical-chemical properties of BPA are described below (Staples et al., 1998; Cousins et al., 2002; ECB 2008). Bisphenol A is a moderately polar substance (log Kow 3.3-3.5) with good solubility in most organic solvents and moderate solubility in water $\left(300 \mathrm{mg} / \mathrm{L}\right.$ at $\left.25^{\circ} \mathrm{C}\right)$. It has a high boiling point and a low vapour pressure at ambient temperatures. BPA has two unhindered phenolic hydroxyl groups and hence, it exhibits chemical properties typical of simple phenols, such as a slightly acid character and susceptibility to oxidation. At physiological $\mathrm{pH}$, the BPA molecule is predominantly in the non-ionised form. The two phenolic hydroxyls are the main reaction centres of BPA and products derived from BPA involve reactions via these groups.

Main physical-chemical properties of BPA.

$\begin{array}{ll}\text { Physical State at STP: } & \text { White solid flakes or powder } \\ \text { Melting Point: } & 155-157^{\circ} \mathrm{C} \\ \text { Boiling point: } & 360^{\circ} \mathrm{C} \text { at } 1013 \mathrm{kPa} \\ \text { Vapour pressure: } & 5.3 \times 10^{-9} \mathrm{kPa} \text { at } 25^{\circ} \mathrm{C} \\ \text { Solubility in water: } & 300 \mathrm{mg} / \mathrm{L} \text { at } 25^{\circ} \mathrm{C} \\ \text { Octanol-water partition coefficient: } & \mathrm{Log} \mathrm{Kow} \mathrm{3.3-3.5} \\ \text { Acid dissociation constant: } & \mathrm{pK}_{\mathrm{a}} 9.6-11.3\end{array}$

\subsection{Overview of existing assessments on BPA}

\subsubsection{Existing assessments}

The toxicological profile of BPA was described in several reports of EFSA $(2006,2010)$, USA National Toxicology Program (NTP-CERHR, 2008), FAO/WHO (2010), ANSES (2011) and in several reviews (Arnich et al., 2011; Hengstler et al., 2011). EFSA opinions issued focused on the oral route of exposure, because it was found to be the most relevant for risk assessment of food/feed. In the 1980s, a series of sub-chronic and chronic studies were performed by the USA-NTP and U.S. EPA (US Environmental Protection Agency), whereby the majority of the studies used the oral route. Doses ranged from 250 to 4000 $\mathrm{ppm}$ (=250 to $4000 \mathrm{mg} / \mathrm{kg}$ feed) corresponding to maximum exposure dose of approximately $400 \mathrm{mg} / \mathrm{kg}$ b.w./ $/ \mathrm{day}^{3}$ in rats and from 5000 to $25,000 \mathrm{ppm}$ ( $=5000$ to $25,000 \mathrm{mg} / \mathrm{kg}$ feed) corresponding to maximum exposure dose of approximately 5600 $\mathrm{mg} / \mathrm{kg}$ b.w./day in mice (US-NTP, 1982). Doses higher than $1000 \mathrm{ppm}(=1000 \mathrm{mg} / \mathrm{kg}$ feed, which corresponds to approximately $100 \mathrm{mg} / \mathrm{kg} \mathrm{b.w./day)} \mathrm{led} \mathrm{to} \mathrm{decreased} \mathrm{body}$ weight in both sexes of rats. Doses higher than $9000 \mathrm{ppm}(=9000 \mathrm{mg} / \mathrm{kg}$ feed $)$, the

\footnotetext{
${ }^{3}$ The indicated dose levels in $\mathrm{mg} / \mathrm{kg} \mathrm{b.w./day} \mathrm{were} \mathrm{calculated} \mathrm{using} \mathrm{the} \mathrm{general} \mathrm{conversion} \mathrm{factor} \mathrm{of} 0.1$ for rats and 0.2 for mice (EFSA 2012a)
} 
total dose depending on the food intake, led to an increase in mean liver weight in dogs (EC 2003).

\subsubsection{Controversial issues}

It is recognized that regarding the risk assessment of BPA after oral uptake, several controversies still need to be addressed.

- In monotonic responses, the effect either increases or decreases over the full dose range tested. It remains unclear whether BPA exhibits a non-monotonic doseresponse, and whether the effect is seen with low doses, but not with high doses (Goodmann et al., 2009; Vandenberg et al., 2012; Rhomberg and Goodman, 2012).

- There are diverging views whether BPA causes adverse effects in humans related to its estrogenic activity at exposure levels present in the population (Borrell, 2010; Aschberger et al., 2010; Taylor et al., 2011; Yang et al., 2009). Increases in prostate weights of $30-35 \%$ compared to controls were observed in $\mathrm{F}_{1}$ adult mouse offspring that were exposed in utero by orally dosing dams with 2 and 20 $\mu \mathrm{g} / \mathrm{kg} /$ day BPA from gestational days 11 through 17 (Nagel et al., 1997). Other studies did not reproduce this result (Ashby et al., 1999; Cagen et al., 1999).

- Developmental exposure to BPA can affect adiposity, glucose or insulin regulation, lipid profiles or other end-points related to diabetes or metabolic syndrome (Miyawaki et al., 2007; Somm et al., 2009; Alonso-Magdalena et al., 2010; Alonso-Magdalena, et al., 2011; Nadal 2013; Ryan et al., 2010; Wei et al., 2011; Mackay et al., 2013; Angle et al., 2013). However, other studies dealing with these endpoints had contradictory results (e.g. Anderson et al., 2013).

- Effects on mammary tissue (e.g. proliferative changes in mammary gland, Ayyanan et al., 2011; Kass et al., 2012; Tharp et al., 2012) in animals were reported but are difficult to apply to humans.

- Neurological, neurodevelopmental and neuroendocrine effects are uncertain because animal studies may not be applicable to humans. A minority view to the EFSA BPA assessment (EFSA 2010) expressed concerns resulting from studies published after 2006 in which animals were exposed during prenatal and postnatal development. The studies appear to indicate that adverse effects, in particular brain receptor programming, immune modulation and susceptibility to breast tumours, might occur at doses below the current No Observed Adverse Effect Level (NOAEL).

- Observational epidemiological studies, suggesting associations between BPA exposure (mainly measured as spot urine concentrations) and chronic health effects such as coronary heart disease, reproductive disorders and others are uncertain, because the design of observational studies does not allow the establishment of causal relationships.

Some controversial issues were recently discussed by Shelnutt et al. (2013) including a summary on the current regulatory status of BPA, a review of recent pharmacokinetic studies and studies on neurobehavioral effects, and how this new information addresses the National Toxicology Program's NTP's 2008 finding of "some concern" (Shelnutt et al., 2013). The species differences in pharmacokinetics of BPA were recognised as was the lack of certain neurobehavioral effects of BPA. In addition, ongoing and planned research in cooperative studies on BPA between NTP/FDA and National Institute of Environmental Health Sciences (NIEHS) in the USA were described (Shelnutt et al., 2013). 


\subsubsection{Conclusion}

The exposure to and toxicity of BPA have been investigated in depth by a multitude of published studies. In risk assessments carried out to date, the focus was not on BPA via non-oral routes (e.g. subcutaneous, dermal).

In existing evaluations, the following are conclusions for the oral route of exposure to BPA:

- NOAEL of $5 \mathrm{mg} / \mathrm{kg}$ b.w./day in rats

- Tolerable Daily Intake (TDI) of $50 \mu \mathrm{g} / \mathrm{kg}$ b.w.

- Developmental toxic effects only observed at doses with severe maternal toxicity in rats and mice

- An overall NOAEL for reproductive toxicity of $50 \mathrm{mg} / \mathrm{kg} \mathrm{b.w./day} \mathrm{in} \mathrm{rats} \mathrm{and} \mathrm{mice}$

- In terms of toxicokinetics, there is a difference between rats and humans (the latter presenting a shorter half-life) as well as between the oral and the parenteral route of exposure

- Due to the first pass effect, after oral uptake, the systemic exposure to free BPA is a small fraction of the external dose in all species

- Unresolved issues remain in the risk assessment of BPA after oral and subcutaneous uptake

In its recent evaluation, EFSA (EFSA 2015) included both the oral and dermal (via thermal paper) exposure to BPA and derived a temporary TDI (t-TDI) of $4 \mu \mathrm{g} / \mathrm{kg}$ b.w./day. EFSA used for the derivation of the t-TDI a Benchmark dose modelling approach and derived a $\mathrm{BMDL}_{10}$ value of $8960 \mu \mathrm{g} / \mathrm{kg} \mathrm{b.w./day} \mathrm{based} \mathrm{on} \mathrm{effects} \mathrm{on} \mathrm{the}$ kidney in multigeneration reproductive toxicity study in mice using oral exposure (Tyl et al., 2008). Similar results (liver and kidney toxicity) were obtained in a rat multigeneration study (Tyl et al., 2002). Notably, the internal exposure of the organs differs: hepatic exposure is presystemic, whereas renal exposure is systemic. The doses at the site of action (i.e. liver and kidney) differ after the oral route of exposure because of the biotransformation occurring in the liver/small intestines which results in a lower dose of free BPA for kidney exposure. However, this is different for non-oral routes of exposure for which BPA may be directly systemically available. The obtained $B M D L_{10}$ dose, estimated to be the low dose inducing a $10 \%$ deviation compared to control animals, was translated into a human dose inducing similar effects, the human equivalent dose (HED). The HED was determined considering the ratio of internal exposure in mice (the species treated with BPA in the study used for the $B M D L_{10}$ derivation) versus the internal exposure in humans based on toxicokinetic studies. So, the $\mathrm{BMDL}_{10}$ of $8960 \mu \mathrm{g} / \mathrm{kg} \mathrm{b} . \mathrm{w}$./day in mice translates to a HED of $609 \mu \mathrm{g} / \mathrm{kg} \mathrm{b}$.w./day. An uncertainty factor of 25 could be established based on the following considerations: 1 (as the HED is accounting for interspecies differences in kinetics) $\times 2.5$ (interspecies dynamic factor) $\times 10$ (intraspecies factor) $=25$. An additional uncertainty factor of 6 was applied as a result of the uncertainty analysis related to mammary gland, reproductive, neurobehavioural, immune and metabolic system effects. Therefore, a BPA specific total uncertainty factor of 150 was applied resulting in the t-TDI of $4 \mu \mathrm{g} / \mathrm{kg} \mathrm{b}$.w./day. In view of the still present uncertainties, the TDI was declared temporary.

The SCENIHR considers the temporary TDI (t-TDI) of $4 \mu \mathrm{g} / \mathrm{kg}$ b.w./day by EFSA which includes uncertainties expressed as a BPA specific assessment factor of 150, a solid base for carrying out a BPA risk assessment for BPA-containing medical devices. 


\subsection{I dentification of the relevant medical devices}

\subsubsection{Medical devices}

Medical devices produced with PC and polysulfone, due to their chemistry, may contain BPA residues, whereas others like PVC may or may not contain BPA residues depending on their production method. In addition, other BPA-derivatives (such as epoxy resins) may be used in dental materials (Fig. 1). Annex I lists medical devices that may contain BPA.

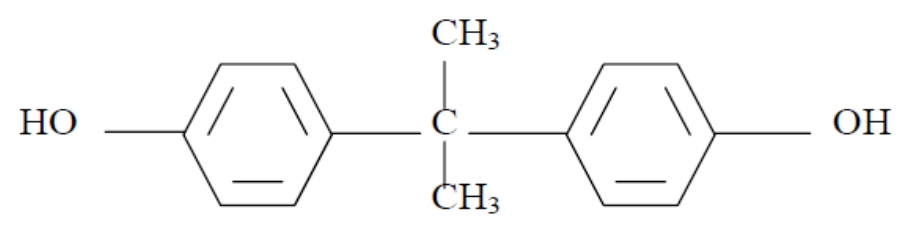

BPA<smiles>CC(C)(c1ccc(OCC2COC2)cc1)c1ccc(OCC2CO2)cc1</smiles><smiles>C=C(C)C(=O)OCC(O)COc1ccc(C(C)(C)c2ccc(OCC(O)COC(=O)C(=C)C)cc2)cc1</smiles>

BISGMA

BISDMA<smiles>C=C(C)C(=O)OCCOc1ccc(C(C)(C)c2ccc(OCCOC(=O)C(=C)C)cc2)cc1</smiles>

Bis-EMA

Figure 1: BADGE: Bisphenol A diglycidyl ether; Bis-GMA: Bisphenol A glycidyl methacrylate; Bis-DMA: Bisphenol A dimethylacrylate; Bis-EMA:ethoxylated bisphenol A dimethacrylate 


\subsubsection{BPA presence in and release from medical devices}

Potential exposure to BPA from PC products (or articles, as defined under REACh) ${ }^{4}$ can derive from the incomplete polymerization of the monomer during the manufacturing process as well as from the breakdown or hydrolysis of the polymer under certain conditions (ECB 2003, EC 2010a, Mercea 2009). It is known that polymerization of monomers is rarely complete, and un-reacted monomers are almost always released from polymer resins (Begley et al., 1990, 2005; De Meulenaer and Huyghebaert 2004). The major factor influencing residual amounts of BPA is the presence of water in the PC before processing, the use of excessively high processing temperatures and the use of additives that promote degradation. Residual BPA in PC is likely to be low when proper processing and handling conditions are used. For instance, in PC articles used for food contact (e.g. bottles, food containers), the residual content is usually less than $10 \mu \mathrm{g} / \mathrm{g}$ of PC (ECB 2003). The presence of residual BPA in PC is illustrated by the migration of BPA mainly into water, $3 \%$ acetic acid, $10 \%$ aqueous ethanol, and olive oil, but also into foods, with levels of up to $50 \mu \mathrm{g} / \mathrm{L}$ of BPA in infant formula from baby bottles (EC 2010b).

Breakdown or hydrolysis of the PC polymer after manufacturing can occur which produces free monomer.. Factors affecting BPA release from PC used in food contact materials have been studied and reviewed (Aschberger et al., 2010; EC 2010b; Beronius and Hanberg, 2011; Geens et al., 2012). Length of contact time, high temperatures and high $\mathrm{pH}$ (hydroxide aqueous solutions) increase BPA release. Additionally, residual alkaline detergent remaining on the surface of the polymer after dishwashing may increase the release of BPA and reactions with amines and ethanol of PC were observed (Maia et al., 2010; Sajiki \& Yonekubo, 2004; Bileset al., 1997; Jie et al., 2006). It remains unclear whether other releasing facilitators such as the composition of mineral water or repeated use (ageing) of the articles increase BPA release.. Importantly, studies on BPA content and its leaching from PC medical devices upon use are limited.

Polysulfones are polymers obtained by reaction between a diphenol and bis(4chlorophenylsulfone) producing an ether-oxide. BPA is one of the diphenols used for the production of PSU and if used during this reaction, BPA leaching is then possible. These polymers are mostly used in medical devices as membranes, especially in hemodialyzers.

Polycarbonate pellets used for the manufacture of medical devices

Haishima et al. (2001) found a total BPA content of 4.0 and $7.2 \mathrm{mg} / \mathrm{kg}$ in two types of PC pellets, using tetrahydrofuran for the dissolution of the polymer. One type of polysulfone(PSU) was analysed resulting in a significantly higher value: $34.5 \mathrm{mg} / \mathrm{kg}$. Furthermore, 2 PC casings lacking hollow fibers were extracted with water and methanol (10 $\mathrm{mL}$ each, $16 \mathrm{~h}$ ) at room temperature, while shaking. The extraction power of methanol was evident from the results: BPA released was 11.7 and $13.7 \mathrm{ng} /$ casing by water extraction, and 296 and $345 \mathrm{ng} /$ casing by methanol extraction.

Data on BPA content in medical devices and/or PC pellets used for the manufacturing of medical devices were submitted in the Call for Information ${ }^{5}$. Low amounts of BPA were observed in PC pellets. The amount of total BPA extracted (24h soxhlet extraction in isopropanol) from PC pellets (3 replicates) used to manufacture medical devices, as well as from finished trocar tubing was $0.2-0.3 \mathrm{mg} / \mathrm{kg}$. No significant difference was found in the amount found in the pellets and in the finished trocar sleeve device. However, BPA

${ }^{4}$ An article is defined within REACh guidance documents (RIP 3.8) as an object with physical properties more important to its function than any chemical properties.

${ }^{5}$ For the data submitted in response to the Call for Information the information was available as far as provided by the applicant and complete study reports were not available for evaluation by SCENIHR. 
could not be detected when using a $0.9 \%$ sodium chloride solution at $37^{\circ} \mathrm{C}$ for extraction time intervals from $1 \mathrm{~h}$ up to $168 \mathrm{~h}$ (Limit of Detection, LOD, approximately $2 \mathrm{mg} / \mathrm{kg}$ ) using conditions to mimic human physiological conditions. Notably, the LOD for saline analysis is 10 times higher than the amount observed using soxhlet extraction in isopropanol. Therefore, the assumption that $100 \%$ of BPA is released in one day represents the worst-case scenario.

BPA could be extracted $(4-5.8 \mathrm{mg} / \mathrm{kg}$ ) only from PC-drinking cups. In PC pellets and a PC-containing trocar, no BPA was detected in a follow-up study of medical grade PC when extracted with isopropyl alcohol and ethanol to account for potential accumulation in blood lipids (LOD approximately $0.5 \mathrm{mg} / \mathrm{kg}$ material). After sterilization by gamma radiation, similar results were obtained using the same PC-containing materials (submitted through the Call for Information).

\section{Medical Devices used for air and/or gas circulation}

In hoods of neonatal incubators and neonatal intensive care units (NICU), BPA could not be detected (LOD approximately $50 \mu \mathrm{g} / \mathrm{m}^{3}$, ISO $16000-6$ method) in the analyzed gases after a prolonged period of contact time of the material with the breathing gas ${ }^{5}$.

\section{Hemodialyzers}

Several studies have reported the leaching of BPA from hemodialyzers, though the number of devices in each study was limited. Four hemodialyzers, composed of a combination of PC casing and cellulose acetate hollow-fibers (1 device), PC casings and PSU (polysulfone) fibers (2 devices), and polystyrene and PSU (1 device), were tested for the amount of BPA released (Haishima et al., 2001). Water and bovine serum $(250 \mathrm{~mL}$ each), the latter used as a simulant for human blood circulating into hollow-fibers during hemodialysis, were circulated at $10 \mathrm{~mL} /$ minfor $16 \mathrm{~h}$ at room temperature in the four devices tested. BPA recovered ranged from 3.78 to $141.8 \mathrm{ng} /$ module using water circulation and from 140.7 to $2090 \mathrm{ng} /$ module when bovine serum was used. The highest values of BPA released corresponded to the 2 hemodialyzers tested consisting of PC casings and PSU fibers were 1 and $2 \mu \mathrm{g} /$ module. Moreover, a $17.2 \%(\mathrm{v} / \mathrm{v})$ ethanol solution was found to extract comparable BPA amounts as with bovine serum, reaching the maximum release after 2 to $4 \mathrm{~h}$ circulation.

BPA concentrations of $83.3 \mathrm{ng} / 10 \mathrm{mg}$ and $122.5 \mathrm{ng} / 10 \mathrm{mg}$ in PSU and PEPA (polyesterpolymeralloy) hollow fibers, respectively, were reported (Murakami et al., 2007). The hollow fibers, taken from individual dialyzers, were crushed and dissolved in hexane.

Fink (2008) investigated the leaching of substances (including BPA) from five different types of dialyzers and PVC blood tubing. All the dialyzers were composed of either PC or PSU: PC housing and PSU-PVP blend membranes $(n=2), P C$ housing and polyamide-PSU blend membrane $(n=1)$, and PP housing, PSU-PVP blend membrane $(n=2)$. The surface area range was $1.3-1.8 \mathrm{~m}^{2}$. The blood and dialysate compartment of each dialyzer was connected by $110 \mathrm{~cm}$ standard PVC tubes. Dialysis was simulated using two different eluents (volumes ranging $230-410 \mathrm{~mL}$ ), reverse osmotic water and $17.2 \%$ ethanol, the latter as a substitute of bovine serum (hence, simulating human serum). Temperature, dialysis period and flow rate were adjusted to represent realistic dialysis modalities: $37^{\circ} \mathrm{C}, 230 \mathrm{~mL}$ eluate/min for $4 \mathrm{~h}$ and also for $24 \mathrm{~h}$ as the worst-case scenario. Three independent eluates with new dialysers each time were obtained at each elution condition. BPA was quantitated in all eluates by LC-MS/MS.

In agreement with the study of Haishima et al. (2001), higher levels were measured when $17.2 \%$ ethanol was used (to simulate blood), ranging from 54.8 to 4299 $\mathrm{ng} /$ dialyser, whereas BPA levels were $6.4-71.3 \mathrm{ng} /$ dialyser using water as eluent (Fink 2008). Other factors influencing the amount of leaching BPA were the type of dialyzer, different batch of the same type, the size of the membrane surface and time of dialysis. In general, the longer the dialysis time, the greater the leaching. Additionally, an increase in the surface area, when comparing dialyzers with the same material for housing and membranes resulted in a higher amount of BPA extracted. The maximum of 
leaching BPA was estimated to be $4.3 \mu \mathrm{g} /$ dialyzer when circulating $17.2 \%$ ethanol for $24 \mathrm{~h}$ (3.4 $\mathrm{\mu g} /$ dialyzer for $4 \mathrm{~h}$ circulating time). Contribution of the PVC tubing to total BPA content in the eluates was negligible, and the levels found were below the limit of quantification $(3.42 \mathrm{ng} / \mathrm{mL})$. The results obtained with water and with $17.2 \%$ ethanol were in the range reported in the previous study by Haishima et al. (2001), except for a single batch on a type of dialyzer, which released almost 2-fold higher levels than the highest levels reported by Haishima et al. (2001). However, this discrepancy could be attributed to different conditions used in both studies; temperature, duration and flow.

Krieter et al. (2013) also reported release of BPA from 3 different dialyzers, one with a $1.7 \mathrm{~m}^{2}$ high-flux polyethersulfone membrane, and two with $1.3 \mathrm{~m}^{2}$ polysulfone membranes, high and low flux, respectively. All dialyzers had PC housing. BPA-free sterile water $(400 \mathrm{~mL})$ was circulated through the blood and dialysate compartments for $3 \mathrm{~h}$, at $250 \mathrm{~mL} / \mathrm{min}, 37^{\circ} \mathrm{C}$ and BPA was measured by ELISA. As found in other studies, eluted BPA concentrations differed significantly between dialyzers, averaged $(n=6)$ levels found being $140.8 \pm 38.7,48.1 \pm 7.7$ and $6.2 \pm 2.5 \mathrm{ng} /$ dialyzer. These results are in the range with those reported in other studies when using water as eluent (Hashima, 2001; Fink, 2008). The highest BPA levels were eluted from the low-flux dialyzer with PSU membrane, and the lowest from the dialyzer with polyethersulfone membrane.

Leaching of BPA from PSU hollow fiber membranes used in hemodialyzers and hemoconcentrators has been recently reported (Cho et al., 2012). The authors studied the kinetic elution profile in ten multiple consecutive extractions with $1 \mathrm{~L}$ of $17.2 \%$ ethanol at a flow rate of $200 \mathrm{~mL} / \mathrm{min}$, for one hour. Every hour the ethanolic solution was refreshed with a new one. The experimental results were fitted to derive an equation to predict the total leachable BPA. For a PSU membrane with a total surface area of $0.5 \mathrm{~m}^{2}$ and weight of $7.9 \mathrm{~g}$, the total leachable amount of BPA was estimated as $20.7 \mu \mathrm{g}$, and $95 \%$ of this was released after 10 consecutive extractions. Single prolonged $(6 \mathrm{~h})$ extraction tests (same conditions) were also performed on PSU membranes with surface areas of $0.4 \mathrm{~m}^{2}$ and $0.7 \mathrm{~m}^{2}$, respectively. BPA concentrations were measured (LC/MS) at different time points from 0.33 up to $6 \mathrm{~h}$. In agreement with the Haishima (2001) study, released BPA reached a plateau in approximately $2 \mathrm{~h}$ (read values from the figure in the publication are $1.3-1.4 \mathrm{ng} / \mathrm{mL}$ and $0.65-0.7 \mathrm{ng} / \mathrm{mL}$ for the $0.7 \mathrm{~m}^{2}$ and $0.4 \mathrm{~m}^{2}$ PSU membranes, respectively). If the flow rate was decreased to half, the time to reach the equilibrium increased to $4 \mathrm{~h}$, but the final concentration was not dependent on the flow rate of the extraction solution.

\section{Cardiopulmonary bypass}

A study by Sakurai H. (2002, only abstract available in English) indicates the presence of BPA in cardiopulmonary bypass circuits, as BPA leaching was observed during open-heart surgery (see exposure section).

\section{Effect of sterilization on medical devices:}

Sterilization of medical devices is usually performed either by steam, irradiation, gas (ethylene oxide) or "gas-plasma" (hydrogen peroxide). Each method may interact with the content of the medical device, creating some by-products, as sterilization may modify/deteriorate the polymer component of the medical device, which, as a result, may modify the BPA release properties. In the specific case of plastics and polymers, effects are described because long-term and extensive reviews are available in the literature (Mendes et al., 2007; McKeen, 2012). These effects include alteration of resistance, surface modification, release and/or modification of some components (Baker et al., 2000; Brown et al., 2002).

Shintani (2001) reported migration of BPA from four dialyzers into a saline extract for different sterilization methods and for different devices. The procedures used for sterilization were autoclaving $\left(121^{\circ} \mathrm{C}\right.$, usually $\left.15-20 \mathrm{~min}\right)$ and gamma radiation at 25 KGy. BPA levels found were 0.1-0.2 ppb $(\mu \mathrm{g} / \mathrm{L})$ in the saline extract. 
In another study (Shintani et al., 2003), BPA content after extraction in $4 \mathrm{~mL}$ was determined by LC-MS-UV in one PC $(119 \mathrm{mg} / \mathrm{kg})$ and three PSU membranes $(43,207$ and $247 \mathrm{mg} / \mathrm{kg}$ ) sterilized with ozone gas (gas concentration $300 \mathrm{ppm}, \mathrm{RH} 80 \%, \mathrm{~T} 35^{\circ} \mathrm{C}$ ) by extraction with ethanol. BPA was not detected after extraction of unsterilized and steam-sterilized $\left(121^{\circ} \mathrm{C}, 15 \mathrm{~min}\right.$ ) PC and PSU membranes (less than $4-11 \mathrm{mg} / \mathrm{kg}$, based on the weights of the tested membranes which were approximately $20 \mathrm{mg}$ and 7 $\mathrm{mg}$ for PSU and PC, respectively). Sterilization by means of ethylene oxide was not used in this study.

\section{Dental materials}

BPA exposure from dental materials is occurring, especially from dental sealants (Olea et al., 1996), but also from composites and other polymer-based restorative materials (Van Landuyt et al., 2011, 2013). Therefore, BPA is not a component in dental materials ${ }^{6}$, but may be present as a contaminant or degradation product. Some dental materials are produced using monomers synthesized from BPA, such as Bis-GMA, Bis-EMA and incidentally Bis-DMA (all methacrylates), and BADGE (epoxy), see Figure 1. Only BisDMA, which has an ester linkage, can be hydrolysed to release BPA. The ether linkage in Bis-GMA is stable (Schmalz et al., 1999). The reports on BPA leaching from dental materials vary extensively, but it seems that only a few specific products/brands were detected as a source of BPA (Arenholt-Bindslev, 1999). One study analyzing 28 different materials found BPA in only one of them (Lewis, 1999).

In 2011, Van Landuyt and co-workers published a systematic review of 71 publications dealing with the release of substances from resin-based materials of which 11 studies investigated the release of BPA. They considered exclusively in vitro incubation in aqueous and organic solvents, for at least 24 hours, without a pre-incubation process. The review reported a few studies with BPA-release in water-based solutions, the highest individual value being $67 \mathrm{nmol} / \mathrm{mm}^{2}$ surface area of the resin bonding material as published by Mazzaoui et al. (2002). Notably, this amount was measured on the resin bonding material, which is an adhesive normally not exposed to saliva.

One study (Takahashi et al., 2004) found no release of BPA into water even at elevated temperature $\left(65^{\circ} \mathrm{C}, 24 \mathrm{~h}\right)$ from PC crowns, but release of $0.28 \mu \mathrm{g}( \pm 0.02)$ from $100 \mathrm{mg}$ material into ethanol $\left(65^{\circ} \mathrm{C}, 24 \mathrm{~h}\right)$. The amount of BPA released into other organic solvents was $4.72 \mu \mathrm{g}$ (acetic acid) and $8.80 \mu \mathrm{g}$ (acetonitrile), both at $65^{\circ} \mathrm{C}, 24 \mathrm{~h}$. No LOD/LOQ was declared in the study.

\section{Adhesives in orthodontic applications}

Orthodontic treatment involves using fixed or removable appliances (dental braces) to correct the positions of teeth. The success of a fixed orthodontic appliance depends on the attachments (brackets and bands) being attached securely to the teeth so that they do not become loose during treatment. A number of epoxy-resin based adhesives are available to attach bands to teeth. Eliades and co-workers quantitatively characterized in vitro BPA released from orthodontic adhesives after artificial accelerated aging (Eliades et al., 2007). No trace of BPA was identified for either adhesive across all time intervals, implying that, if present, the amount of BPA did not exceed the detection limit of the analytical technique $(0.1 \mathrm{mg} / \mathrm{kg}$ of adhesive).

Eliades and co-workers also quantified BPA released from a light-cured orthodontic adhesive used to bond lingual fixed retainers (Eliades et al., 2011). Eighteen recently extracted premolars, divided into 3 groups of 6 teeth each, were embedded in plaster in an arch shape. A light-cured adhesive was bonded to a 3-strand, heat-treated twist flex wire adjusted to the lingual surface of the teeth, and the arches were immersed in

\footnotetext{
${ }^{6}$ At least one manufacturer has actively informed during the call for information that their products have no additions of BPA:

http://multimedia.3m.com/mws/mediawebserver?mwsId=66666UF6EVsSyXTtNXTEnxTEEVtQEVs6E Vs6EVs6E666666--\&fn=bpa letter.pdf
} 
double-distilled water for 10, 20 and 30 days. The concentration of BPA in the 3 extracts was investigated with gas chromatography-mass spectroscopy; all assays were performed in triplicate, and the results were averaged. Measurable amounts of BPA were identified for all groups, with the highest value $(2.9 \mu \mathrm{g} / \mathrm{L})$ found in the immersion media of the 30-day groups (six teeth embedded in plaster and arranged in an arch mimicking the shape of of the six mandibular anterior teeth), whereas the control (tooth storage solution) had $0.16 \mu \mathrm{g} / \mathrm{L}$. The total release could not be determined, because the incubation volume was not presented in the paper. The level in the control samples indicates background levels of BPA in the lab equipment used. In conclusion, BPA released from a light-cured adhesive used to bond lingual fixed retainers might be assigned to the application mode of the material that differs from conventional use.

As orthodontic bonding resins are exposed to oral fluids and are in contact with tissues throughout treatment, leaching from resin can occur at 2 times: during the setting period of the resin and later when the resin is degraded. Leaching during the first process is related to the degree of conversion. Sunitha et al. (2011) used high-performance liquid chromatography to assess BPA released from an orthodontic adhesive with various lightcuring tip distances and to correlate the release to the degree of conversion. BPA release was greater in specimens cured with a greater light-curing tip distance. The degree of conversion decreased with increased light-curing tip distances. A negative correlation was found between BPA release and degree of conversion.

Watanabe (2004) analyzed the leaching of BPA in water at $37^{\circ} \mathrm{C}$ for $12,16,25$, and 34 months from brackets, denture base and temporary crown PC materials. The total BPA released was found to be $37.4( \pm 3.56), 2.2( \pm 0.03)$, and $2.8( \pm 0.32) \mu \mathrm{g} / \mathrm{g}$, respectively. The BPA content in the materials was shown to increase after 34 months (max $472 \mu \mathrm{g} / \mathrm{g}$ for brackets), and the MW of the PC to decrease. The BPA content in retrieved PC brackets was in the range $38 \mu \mathrm{g} / \mathrm{g}$ (18 months) to $697 \mu \mathrm{g} / \mathrm{g}$ (40 months).

Watanabe et al. (2001) analyzed (HPLC/UV-DAD or fluorescence) five retrieved PC brackets from five patients for their content of BPA. At retrieval points ranging from 5-15 months, the BPA content in the brackets were found in the range $56-102 \mu \mathrm{g} / \mathrm{g}$ bracket. (One bracket weighs approximately $22 \mathrm{mg}$ ). In addition, they measured the in vitro leaching of BPA from the brackets in water at $37^{\circ} \mathrm{C}$ and $60^{\circ} \mathrm{C}$ for $3,6,9,12$ months and $1,2,4,6,10,14$ weeks, respectively. The release after 12 months reached $3.8 \mu \mathrm{g} / \mathrm{g}$. The release after 14 weeks reached $35 \mu \mathrm{g} / \mathrm{g}$. The BPA content in PC brackets immersed in water increased to a maximum of $434 \mu \mathrm{g} / \mathrm{g}\left(14 \mathrm{wks}, 60^{\circ} \mathrm{C}\right)$. Calculations of exposure ( $3.8 \mu \mathrm{g} / \mathrm{g}, 5-15$ months, 28 brackets, $50 \mathrm{~kg}$ b.w.): daily BPA intake of $0.1-0.3 \mathrm{ng} / \mathrm{kg} /$ day, as given by the authors.

Recently, Kloukos et al. (2013) reviewed all the publications on BPA leaching from orthodontic adhesive resins and PC brackets. The objective of this systematic review was to assess the short- and long-term release of components of orthodontic adhesives and PC brackets in the oral environment. Eleven studies met the inclusion criteria and all were observational studies conducted in vivo or in vitro. The BPA released from orthodontic bonding resins was found to be between 0.85 and $20.88 \mathrm{ng} / \mathrm{mL}$ in vivo and from traces to $65.67 \mathrm{ppm}(\mathrm{mg} / \mathrm{L})$ in vitro. Polycarbonate brackets released amounts of $22.24 \mu \mathrm{g}$ per gram in ethanol solution and $697 \mu \mathrm{g}$ per gram after 40 months in water. The available evidence on this topic derived from observational in vivo and in vitro studies that represent a moderate level of evidence. The variety of setups and the different units allied to the diversity of reporting among studies did not allow calculation of pooled estimates (Table 1). 
Table 1: BPA released in experimental media (in vitro)

\begin{tabular}{|c|c|c|c|}
\hline Reference & $\begin{array}{l}\text { Orthodontic } \\
\text { material }\end{array}$ & Time at sampling & BPA concentration \\
\hline $\begin{array}{l}\text { Sunitha et al., } \\
2011\end{array}$ & $\begin{array}{l}\text { Light-cured } \\
\text { adhesive resin }\end{array}$ & Day 7 & $65.67 \mathrm{ppm}(\mathrm{mg} / \mathrm{L})$ \\
\hline $\begin{array}{l}\text { Eliades et al., } \\
2011\end{array}$ & $\begin{array}{l}\text { Light-cured } \\
\text { adhesive }\end{array}$ & 30-days exposure & $\begin{array}{l}2.9 \mu \mathrm{g} / \mathrm{L} \\
\text { (control group: } 0.16 \mu \mathrm{g} / \mathrm{L} \text { ) }\end{array}$ \\
\hline Watanabe 2004 & PC brackets & $\begin{array}{l}34 \\
(\text { immersion in water, } \\
37^{\circ} \mathrm{C} \text { ) }\end{array}$ & $37.4 \mu \mathrm{g} / \mathrm{g}$ \\
\hline $\begin{array}{l}\text { Watanabe et al., } \\
2001\end{array}$ & PC brackets & $\begin{array}{l}12 \text { mo. } 37{ }^{\circ} \mathrm{C} \\
\text { Immersion in water } \\
14 \text { weeks } 60 \quad{ }^{\circ} \mathrm{C} \\
\text { Immersion in water }\end{array}$ & $\begin{array}{l}3.8 \mu \mathrm{g} / \mathrm{g} \\
35.0 \mu \mathrm{g} / \mathrm{g}\end{array}$ \\
\hline $\begin{array}{l}\text { Suzuki et al., } \\
2000\end{array}$ & $\begin{array}{l}\text { PC } \\
(n=4)\end{array}$ & $\begin{array}{l}\text { Crushed brackets in } \\
\text { ethanol solution }\end{array}$ & $22.24 \mu \mathrm{g} / \mathrm{g}$ \\
\hline
\end{tabular}

Notably, with a mean weight of approximately $40 \mathrm{mg}$ and a maximum number of 24 applications, the total weight of brackets potentially applied is approximately $1 \mathrm{~g}$ material.

\section{Bone cements}

Bi-functional methacrylates based on bisphenol-A-glycidyldimethacrylate (BisGMA) or triethylene glycol dimethacrylate (TEGDMA) may also be applied in bone cements (Vallo and Schroeder, 2005). There are commercially available cements that comprise three main resins, BisGMA and ethoxylated BPA dimethacrylate (BisEMA) and TEGDMA as a viscosity modifier and reinforcing particles. As BPA may be present as potential impurity/residue, and tens to hundreds of grams of these bone cements are used per treatment, this may result in considerable BPA release. However, no data exist to date on BPA release from these new bone cement materials.

\subsubsection{Conclusions}

BPA can be present in medical devices as residue from an (incomplete) polymerization process or result from the hydrolysis of the polymer. BPA can be extracted from medical devices consisting of PC and/or polysulfones, the latter mostly being used in the form of membranes. BPA extraction can be performed in vitro with water, methanol or organic solvents resulting in dissolution of the product. Extraction in methanol results in higher release of BPA compared to water extraction. For PC casings BPA release in water was between 11 and $14 \mathrm{ng} /$ casing, while in methanol the release was between 296 and 345 $\mathrm{ng} /$ casing. Results for PC pellets used for the production of medical devices were 4 to 7 $\mathrm{mg} / \mathrm{kg}$ after dissolution of the pellets. In the Call for Information, for PC drinking cups values of $4-6 \mathrm{mg} / \mathrm{kg}$ were submitted. So, PC used for the production of medical devices seems to have BPA levels similar to those of PC commonly used as food contact materials.

In hemodialyzers, water and bovine serum circulation resulted in a BPA recovery of 4 to $142 \mathrm{ng} /$ module for water and 141 to $2090 \mathrm{ng} /$ module for bovine serum, again indicating that water is not the best medium for BPA extraction. This was confirmed by other data showing BPA release of 6 to $71 \mathrm{ng} /$ dialyzer in water and 55 to $4300 \mathrm{ng} /$ dialyzer in $17.2 \%$ 
ethanol. Low water extraction was observed for three different dialyzers was 141, 48 and $6 \mathrm{ng} /$ dialyzer, respectively. In hollow fibers isolated from individual dialyzers and dissolved in hexane, BPA content was 8.3 to $12.2 \mu \mathrm{g} / \mathrm{g}$ material. The highest values of BPA released corresponded to the 2 hemodialyzers tested that consisted of PC casings and PSU fibers were 1 and $2 \mu \mathrm{g} /$ module. After sterilization procedures, some BPA may have already been released from the dialyzers.

The highest quantity of BPA for dental materials measured was $67 \mathrm{nmol} / \mathrm{mm}^{2}$, which amounts to $15 \mu \mathrm{g} / \mathrm{mm}^{2}$ for a resin bonding material that is commonly not exposed to saliva. For PC orthodontic brackets, the BPA release varied between $22 \mu \mathrm{g} / \mathrm{g}$ (crushed brackets) to $697 \mu \mathrm{g} / \mathrm{g}$ retrieved after 40 months of use by patients. In general, there was limited information provided to assess the reliability of available data.

\subsection{Exposure scenarios}

\subsubsection{Knowledge on BPA exposure}

Most people are exposed to BPA through the diet. BPA in food and beverages accounts for the majority of daily human exposure (Wilson et al., 2007; Chapin et al., 2007; Vandenberg et al., 2007; EFSA 2013). BPA may migrate into food from food and beverage containers with internal epoxy resin coatings and from consumer products made of PC plastic such as tableware, food containers, and water bottles. Polycarbonate was used in the production of baby bottles, but it was discontinued subsequent to European regulation. BPA exposure results from either the release of un-polymerized monomers or the slow decay of polymer bonds in PC leading to monomer release into proximal foods and liquids. Air, dust, and skin contact with thermal paper, are other possible sources of BPA exposure. More recently, EFSA estimated oral equivalent BPA doses due to contact with thermal paper that was deemed to be two to three times higher than the dietary exposure for adolescents and adults (EFSA 2015). Measured concentrations of BPA in human blood, urine and other tissues have indicated that the majority of the population (91-99\%) has detectable levels of BPA-conjugates in their urine, confirming that exposure is widespread in the human population (Vandenberg et al., 2007; Calafat et al., 2008). Some studies indicate the presence of free BPA in blood; however, their reliability and the toxicological relevance is a subject of intense academic and public debate (Calafat et al., 2005; Kang et al., 2006; Vandenberg et al., 2007; Dekant and Völkel, 2008; Calafat et al., 2008; Becker et al., 2009; Bushnik et al., 2010).

To date, there are limited data available on potential exposure to BPA from the use of medical devices (Beronius and Hanberg, 2011).

\subsubsection{Methods for measurement of internal exposure in humans}

Biomonitoring directly measures human daily or cumulative exposures to xenobiotics from all sources by the determination of biological fluids (blood, urine, breast milk, saliva) or tissue concentrations of the chemical or its metabolites.

Regarding BPA, the analytical method used to detect both the parent compound and its metabolites is crucial, especially at the low levels expected in biological samples, and may represent a relevant source of differences among available studies. For biomonitoring studies, independently of the detection method applied, the use of a stable isotope-labeled BPA as an internal standard was suggested (WHO 2010) as the best means to determine the effect of complex matrices (i.e. biological samples) and the stability of BPA during analysis. The use of stable isotope-labeled BPA prevents 
discrepancies due to contamination from external sources during sample collection and processing (see below).

The features of different methods have been summarized by the FDA (US FDA, 2010b): it appeared that 1) among the methods for BPA analysis, the Mass Spectrometry (MS)based methods are considered the best and 2) data obtained by using immunochemical methods (ELISA, RIA) are the least reliable because of low sensitivity, poor selectivity due to cross reactivity with other phenols, and strong matrix effects (especially for urine samples). Even when cross-reactivity with endogenous steroids and most analogous BPA phenolic structures were reduced (Kaddar et al., 2009), immunoassays tend to overestimate serum BPA concentrations when compared to LC-MS/MS data.

A recent development is a new specific radioimmunoassay for the direct measurement of BPA-glucuronide in urine without hydrolysis that requires only the elimination of unconjugated BPA by one solvent extraction step (Harthé et al., 2012). A polyclonal antiBPA antibody showing a $95 \%$ cross-reactivity with BPA-glucuronide and insignificant cross-reactivity with most phenolic structures analogous to BPA was used. The sample preparation phase can significantly reduce external contamination. The method is reported to be valid, precise and accurate in the range of $0.05 \mathrm{mg} / \mathrm{L}$ to $5 \mathrm{mg} / \mathrm{L}$, with a LOD comparable to GC and LC methods. It was reported that BPA-conjugate concentrations measured with the radioimmunoassay method correlated with total BPA concentrations measured by GC/MS in 32 urine samples ( $r=0.86$ ) (Harthé et al., 2012).

GC/MS methods for BPA analysis in blood and urine have been widely used (US FDA, $2010 \mathrm{~b}$ ), although they require derivatization or esterification of BPA (which is a nonvolatile chemical). There is a time-consuming sample preparation phase with different extraction and pre-concentration procedures that may increase the possibility of external contamination of the sample itself (e.g. by plastic disposable lab devices or solvents). Recently, classic GC-MS method based on solvent and solid phase extraction followed by derivatization was adapted and validated for determination of BPA also in tissue samples, such as human placental and fetal liver tissue (Zhang et al., 2011) and in the human maternal and umbilical cord blood serum (Kosarac et al., 2012).

In contrast, HPLC methods can be used to analyse BPA without any pre-derivatization step (Inoue et al., 2001; Inoue et al., 2003).

An LC/MS-MS method was developed to quantify both free and conjugated BPA in blood and urine samples against an internal standard ( $d^{14}$-bisphenol A-glucuronide) (Völkel et al., 2005) and further modified to analyse different matrices (i.e. breast milk) (Ye et al., 2006). $D^{16}$-BPA or ${ }^{13} C_{12}$-BPA can be used as an internal standard. The LOD of LC-based method is in the range of 0.1-0.4 ppb $(\mathrm{ng} / \mathrm{g})$ in blood or urine samples.

With both GC and LC-based methods, an enzymatic hydrolysis step with betaglucuronidase is usually carried out, as BPA-glucuronide is not generally available as an analytical standard. The requirement for two separate assays may lead to critical pitfalls in terms of accuracy and external contamination (see below). To overcome these problems, BPA-glucuronide may be isolated from urine samples, purified by flash chromatography and characterized by mass spectrometry and NMR. The isolated BPAglucuronide was found to be suitable as analytical standard for the simultaneous quantification of BPA and BPA-glucuronide in biological matrices by UPLC/MS/MS (Lacroix et al., 2011).

A round robin study was performed with four laboratories each having their own extraction, detection and analytical methodsfor the presence of unconjugated BPA and conjugated BPA-glucuronide in human blood (Vandenberg et al., 2014). In the blinded spiked sample analysis, all laboratories were able to distinguish low from high values of unconjugated BPA and BPA-glucuronide. Some differences in performance were noted between the laboratories, while at the end of the study three laboratories had verified methods for the analysis of unconjugated BPA, while two laboratories verified for the analysis of conjugated BPA-glucuronide. A laboratory was considered to be verified for a method when the data obtained were within an acceptable rate of error of $20 \%$ in the 
spiked to reported concentration. Collection materials, reagents, and collection and mass spectrometry procedures were evaluated for the presence of BPA contamination. BPA contamination was only detected in some vacutainer butterfly needles. Samples spiked with BPA-glucuronide were evaluated for the possibility of hydrolysis resulting in unconjugated BPA. All laboratories showed that mainly BPA-glucuronide $(92.2 \%-100 \%)$ was present in the BPA-glucuronide spiked samples. All four laboratories used methods that were able to identify both unconjugated BPA and BPA-glucuronide simultaneously (Vandenberg et al., 2014). Investigation resulted in the direct methods being verified, whereas the indirect methods using enzymatic hydrolysis were not. The authors conclude that the round robin study showed that it is possible to use sensitive and accurate methods for the determination of unconjugated BPA and conjugated BPA-glucuronide in human serum samples. Potential BPA contamination needs to be controlled during sample collection. Hydrolysis of BPA conjugates was not observed in the methods used.

The possibility for BPA contamination during the blood sampling, handling and by equipment used was also demonstrated in a study to the BPA levels in Swedish nursing women (Gyllenhammar et al., 2014).

\subsubsection{Internal exposure to BPA in humans from all routes}

A large number of biomonitoring studies to measure BPA in urine are available from North America, Europe and South-east Asia (for overviews see Dekant and Völkel, 2008; Vandenberg et al., 2007, 2010a, 2010b; US FDA, 2010b; Geens et al., 2012). Importantly, BPA in urine is present in its conjugated form. Urinary biomonitoring data provide information on the internal dose, which is the result of total BPA exposure, independently from the sources: therefore, biomonitoring data in urine accounts not only for dietary exposure, but also for non-food sources (e.g. medical devices, dust, thermal and other kinds of paper).

\section{Methodological issues}

The appropriateness of the study design (i.e. sampling time, most adequate fluid/tissue, appropriate sampling and storage procedures) requires a detailed knowledge of the biotransformation and toxicokinetics of any xenobiotic and/or its metabolites, as well as a suitable analytical method. This is particularly relevant for BPA, with its rapid conjugation and an elimination half-life time of only a few $\mathrm{h}$ in blood. BPA concentrations in blood decrease quickly after exposure (WHO, 2010).

In addition, blood concentrations of total BPA (free plus conjugates) determined at one time point are not representative of an average exposure, because they are strongly dependent on the time of blood sampling with respect to the exposure time. Since BPA urinary excretion is almost complete within $24 \mathrm{~h}$ after exposure and due to less invasive sampling, urine is the matrix of choice for assessing daily exposure to BPA in humans. Similar to blood, single spot urine samples serve as a measure of very recent BPA exposures. Urine BPA levels depend on frequency of food intake, time of sampling after food consumption, the last urination and urine production rate.

A 24-hour pooled urine collection is the most appropriate sample to determine average daily exposure to BPA. However, in large cohorts, the high number of spot urine samples collected will average out variations in urinary concentrations of total BPA among individuals arising from temporal factors within a day. It has been reported that mean values from studies reporting BPA concentrations in spot urine samples with a large number of participants correlate well with those using cumulative excretion over $24 \mathrm{~h}$ (Völkel et al, 2008; WHO, 2010). Recently, Christensen et al. (2012) specifically investigated whether spot sample concentrations of BPA were comparable to daily average concentrations. Overall, spot urinary concentrations of BPA have variability roughly similar with corresponding 24-hour average concentrations obtained from a comparable population, suggesting that spot samples can also be used to characterize 
population distributions of intakes, although caution should be applied in interpreting the high end of spot sample data sets.

To estimate the daily BPA intake, the urinary concentrations of total BPA (free and conjugated form) should be multiplied with the 24-hour urinary output $(\mathrm{mL})$ to get the daily excretion of BPA in $\mathrm{ng} /$ day, considering that excretion is almost complete in $24 \mathrm{~h}$ (Völkel et al., 2002, 2005). In addition to the urinary output, BPA concentration can also be adjusted for daily creatinine excretion or for body weight. The correction for urinary output is generally preferred over creatinine excretion (Lakind and Naiman, 2008), due to its high interindividual variation (over $1000 \%$ ) (Geens et al., 2012). The correction is quite relevant when comparing data obtained in different studies, although the corrections are not always used or reported. However, the urine volume is also related to several other factors such as liquid intake, physical exercise, and individual health and lifestyle factors (WHO, 2010).

No study has investigated whether urinary excretion of BPA and other environmental phenols differed by renal function. However, You et al. (2011) estimated the association between renal function and urinary excretion of BPA and they suggested that urinary excretion of BPA decreased with decreasing renal function. Because the associations might differ by age or sex, further studies are necessary to replicate these results and understand the mechanism.

For risk assessment purposes, both free BPA and its metabolites should be determined, especially because most BPA in human is present as conjugates. Koch et al. (2012) investigated the extent of BPA body burden in the German population from 1995 to 2009 based on 600 24-hour urine samples and corresponding plasma samples from the Environmental Specimen Bank. They concluded that the total BPA in urine is the most appropriate and robust marker for BPA exposure assessment (if controlled for BPA contamination). Data on unconjugated BPA in urine and unconjugated or total BPA in plasma, where contamination or breakdown of the glucuronide cannot be ruled out, are of no value for human exposure assessment.

Another potentially confounding effect in determining free BPA is the deconjugation of BPA due to spontaneous hydrolysis and/or to bacterial contamination of the urine sample which may cause hydrolysis (Schöringhumer and Cichna-Markl, 2007; Ye et al., 2007; Helander and Dahl, 2005).

As far as levels in urine sample are concerned, the reporting of data is a crucial issue for comparing data, which are either reported with or without creatinine-adjustment to correct for urine dilution, or adjusted by using 24-hour urine volume which is not specified. However, a common result is that BPA conjugates (mainly glucuronides) are by far the major BPA form present in human urine.

\section{Possible artifacts in measurements of population exposure to BPA}

The leaching of BPA from PC containers for sampling and storage, as well as plastic disposable lab devices, sample workups and analysis (including HPLC capillary systems and solvents) has been identified as a source of free BPA in biological samples. This kind of contamination, possibly contributing to the concentration of free BPA, has been reported in some studies (Markham et al., 2010; Twaddle et al., 2010; Ye et al., 2011).

A recent survey conducted in France on pregnant women after delivery supported the importance of urine sample collection methodology, and the relevance of possibile sample contamination (Vandentorren et al., 2011). Women who had caesarean sections had much higher levels of free and total urinary BPA than women giving birth naturally, the $95^{\text {th }}$ percentile being $273.9 \mu \mathrm{g} / \mathrm{L}$ vs. $4.2 \mu \mathrm{g} / \mathrm{L}$, with data adjusted for creatinine levels (Vandentorren et al., 2011). Since the analytical methodology was correct and urine samples were stored in polypropylene tubes at $-4^{\circ} \mathrm{C}$ first and then at $-80^{\circ} \mathrm{C}$, prior to be analysed, contamination with BPA from exogenous sources during storage can be excluded. The authors did not clearly describe the way urine was collected. However. it is plausible that for women having caesarean section, urinary collection devices were used, 
suggesting that the source of higher BPA levels could be hospital-based. Indeed, supplemental studies on the release of BPA from catheterization probes of urine at room temperature for 12 and $24 \mathrm{~h}$ clearly indicate the time dependence of free BPA extraction from the probes up to approximately $300 \mu \mathrm{g} / \mathrm{L}$. In this case, since the major source of BPA is external to the body of patients, it can be concluded that measured free BPA was due to contamination rather than to real exposure of patients via medical devices.

The presence of analytical artefacts cannot be excluded in many of the available studies because the appropriate internal controls were not carried out (Twaddle et al., 2010). Background contamination was quantified by Doerge et al. (2010a) and found to be as high as $2 \mathrm{ng} / \mathrm{mL}$ in buffer blanks, which may be responsible for the differences in free BPA levels reported in many studies. This highlights the importance of sample preparation and clean-up in the determination of traces of BPA in complex matrices such as biological samples to remove interfering matrix compounds and to increase selectivity by using solvent extraction or SPE clean up or a combination of sol-gel immunoaffinity columns containing anti-BPA antibodies (Cichna-Markl, 2012). Results obtained with the administration to animals or volunteers of ${ }^{14} \mathrm{C}$-labelled or deuterated BPA are not affected by background contamination and are the most reliable methods to perform the BPA toxicokinetics studies and as input for the risk assessment. For measurements not using isotope labelled BPA, a quality control on possible BPA contamination due to sampling equipment and consumables used within the assay is necessary.

In addition, the variability of results obtained in biomonitoring studies to population exposure of BPA depends also on human inter-individual differences, which include age, diet, presence of pathologies (e.g. renal failure, hepatic dysfunction), genetic factors (e.g. polymorphisms of UDPGT enzymes) as well as the pattern of exposure. Especially during medical treatment, additional exposure to BPA may occur when using medical devices consisting of PC components.

\section{Human data on BPA exposure}

Much of the concern for BPA exposure has come from studies reporting relatively high levels of free BPA in human body fluids/tissues (Schönfelder et al., 2002; Fernandez et al., 2007; Lee et al., 2008; Jimenez-Diaz et al., 2010; Zhang et al., 2011; Geens et al., 2012). The presence of high levels of free BPA in human specimens in those studies were questioned on the basis of 1 ) the kinetics of BPA in humans (supported by data in animals, including non human primates) showing $<1 \%$ of total free BPA in serum at peak levels and an almost complete and rapid excretion in human volunteers and 2) the low aggregate intake estimates (mean $<1 \mathrm{microg} / \mathrm{kg}$ b.w./day) made from urinary conjugated BPA measurements (Lakind and Naiman, 2010). This raises questions in data generation and evaluation that should be addressed, underlining the need for a careful quality control to be included in the study design to avoid/minimize any possible source of artefact or external contamination, and confounding factors (Calafat and Needham, 2009; Dekant and Völkel, 2008; Markham et al., 2010; Ye et al., 2007). The major ones are related to i) stability of BPA and BPA conjugates in the sample matrix and during sample processing, ii) BPA background levels due to sample handling and work up, iii) use of appropriate internal standards and analytical methods, including adequate reporting of data.

Most biomonitoring data obtained in different biological samples available up to 2010 have been tabled in a FDA report (US FDA, 2010b) and recently reviewed (Geens et al., 2012). Comparison among results from different studies is not always simple, due to reporting difference. As an example, for statistical analysis non-detectable samples are assigned a value, which is generally, but not always the LOD value, differently determined by the various authors. For this reason all data below LOD and LOQ, as well as some average (median) values should be interpreted with caution.

From blood measurements in a limited number of enrolled individuals, it appeared that free and conjugated BPA levels are typically $\leq 1 \mu \mathrm{g} / \mathrm{L}$ (US FDA 2010b), which is consistent with the known rapid plasma clearance of BPA and its metabolites and kinetic 
studies conducted in humans. Only two studies (Schönfelder et al., 2002; Padmanabhan et al., 2008) reported much higher free BPA levels in maternal and fetal plasma as well as placenta tissue. Because they did not measure conjugates, the reported levels have a limited value and should be interpreted with caution. In a recently performed controlled human study at environmentally relevant BPA doses, where potential technical factors as sources for BPA variability in biological tissues were adequately reduced, serum concentrations of parent BPA were consistently below LOD (1.3 nM) (Teeguarden et al., 2011). The residual observed variability in free BPA levels, approximately by a factor of 4, could be attributed to interindividual variability in BPA metabolic disposition.

Liao and Kannan (2012) determined free and conjugated BPA (glucuronide and disulfate) in human urine and serum samples of 32 healthy volunteers, using solid-phase extraction (SPE) and liquid chromatography-tandem mass spectrometry (LC-MS/MS) techniques. They found $32 \%$ and $19 \%$ of free BPA with respect to the total BPA measured in urine and serum, respectively. Glucuronated BPA, the dominant metabolite, was present in this form in $57 \%$ and $43 \%$ of the total BPA in urine and serum, respectively.

The influence of functionally relevant polymorphic UGT2B15, the major UGT isoform responsible for BPA metabolism, on the blood concentration time profile has been recently investigated by using a validated physiologically based kinetic human model (Partosch et al, 2013). Maximum concentrations (Cmax) and AUCs in blood varied for a factor of 4.7 and 4.6 in high and low metabolisers (dose: $1 \mu \mathrm{g} / \mathrm{kg} / \mathrm{day}$ ) in accordance with biomonitoring data reported by Teeguarden et al. (2011). The highest Cmaxvaluecalculated in the subject with the lowest metabolic clearance is roughly $40 \mathrm{pg} / \mathrm{mL}$, far lowerthan the reported high blood concentrations that cannot be explained by a genetically impaired UGT2B15 activity (Partosch et al, 2013).

The largest-scale studies with a consistently high number of enrolled participants $(n=$ 2517 and 5476 individuals) spread over a broad range of age, were carried out in the USA and Canada, respectively (Calafat et al., 2008; Bushnik et al., 2010).

In both studies, after adjusting BPA levels for creatinine, the youngest age category (611 years) showed the highest urinary concentrations $(3.6 \mathrm{ng} / \mathrm{mL}$ in the US and 1.30 $\mathrm{ng} / \mathrm{mL}$ in Canada), when compared to the average values of the general population (2.6 $\mathrm{ng} / \mathrm{mL}$ and $1.16 \mathrm{ng} / \mathrm{mL}$, respectively). Recent biomonitoring studies in Asian countries produced similar results (Zhang et al., 2011).

According to the WHO (WHO, 2010), the available urinary data allow to estimate the median exposures for adults and for children in the range of $0.01-0.05 \mu \mathrm{g} / \mathrm{kg}$ body weight (b.w.) per day and $0.02-0.12 \mu \mathrm{g} / \mathrm{kg}$ b.w. per day, respectively. Similarly, US FDA derived mean daily intake of BPA is $<0.03-0.13 \mu \mathrm{g} / \mathrm{kg} \mathrm{b.w./day} \mathrm{for} \mathrm{a} 60-\mathrm{kg}$ adult, and 0.07 to $0.12 \mu \mathrm{g} / \mathrm{kg}$ b.w./day for a $36.1-\mathrm{kg}$ child (age 3-14), by using FDAs standard default assumptions (US FDA, 2010a). The earlier evaluation of BPA exposure by EFSA (EFSA, 2013) indicated diet to be the main source of exposure to BPA in all population groups. The more recent evaluation by EFSA also implicated thermal paper as an important source for BPA exposure that could be two to three times higher than the dietary exposure in adolescents and adults (EFSA 2015). In the current EFSA exposure assessment, dietary exposure was up to $857 \mathrm{ng} / \mathrm{kg} \mathrm{b.w./day} \mathrm{for} \mathrm{toddlers} \mathrm{and} \mathrm{up} \mathrm{to} 435$ $\mathrm{ng} / \mathrm{kg}$ b.w./day for infants of 1-5 days. For adults (including woman of childbearing age) the dietary exposure was up to $388 \mathrm{ng} / \mathrm{kg}$ b.w./day. Non-dietary (non-oral) sources contributed with an exposure of up to $1068 \mathrm{ng} / \mathrm{kg} \mathrm{b.w./day} \mathrm{(expressed} \mathrm{as} \mathrm{an} \mathrm{oral} \mathrm{dose).}$

Mose et al. (2012) studied ex vivo the PBA transplacental transfer rate in human placentas. Results lead the authors to conclude that free BPA can cross the placenta by passive diffusion with a transplacental transfer rate of 1 (i.e. the concentration in the fetal blood was equal to the concentration in the blood of the mother), similar to a previous study (Balakrishnan et al., 2010).

Two recent studies reported BPA concentrations (both free and conjugated forms) in amniotic fluid and fetal liver samples (Edlow et al., 2012; Nahar et al., 2012). However, the procedures used to avoid contamination and deconjugation of BPA during sample 
handling were not used, which does not allow any conclusions to be drawn from the study results.

The occurrence of BPA in breast milk was analysed in some small-scaled studies (Cariot et al., 2012; Ye et al., 2006; Ye et al., 2008; Otaka et al., 2003; Sun et al., 2004; Kuruto-Niwa et al., 2007; Yi et al., 2010). This information can be relevant to estimate exposure of breast-fed infants, as a consequence of exposure of the lactating mother. The lactational transfer from the maternal plasma compartment to the maternal milk compartment, both as free and conjugated BPA, has been demonstrated in rat studies (Snyder et al., 2000; Doerge et al., 2010c), where dams were administered a daily oral dose of $100 \mu \mathrm{g} / \mathrm{kg}$ b.w. of ${ }^{14} \mathrm{C}$ - or deuterium-labelled BPA, respectively. The older study indicated that total exposure of the lactating pups was estimated to be approximately $0.3 \%$ of the dose $(\mathrm{mg} / \mathrm{dam}) / \mathrm{kg}$ pup weight, with about $80 \%$ of the total BPA concentration consisting of BPA-glucuronide (EFSA, 2010; Doerge et al., 2010c). The more recent study reported that on day 7 postpartum at 1 hour after dosing, when BPA serum levels are maximal (Doerge et al., 2010a), mean concentrations of $0.2 \mathrm{ng} / \mathrm{mL}$ and $1.7 \mathrm{ng} / \mathrm{mL}$ for free an total BPA, respectively, with a proportion of only $13 \%$ free BPA, were observed. Consequently, the concentrations in pup serum are estimated below 0.2 $\mathrm{nM}(45.6 \mathrm{pg} / \mathrm{ml})$. Therefore, pup exposure via lactation is extremely low $(1 / 300$ of the maternal dose).

BPA has also been reported in human milk. In the most recent study (Cariot et al., 2012), appropriate measures to avoid contamination by environmental BPA were taken, including milk collection (drawn manually without breast milk pumps or gloves), free BPA was absent in solvent blanks. BPA was detected only at low concentrations ( $\leq 0.12 \mathrm{ng} / \mathrm{mL}$; lower than the LOQ) in some of the pooled, mature breast milk samples taken from donors breast-feeding for over 1 month, used as quality-control materials. In the test samples collected from additional donors $(n=3)$ within a few days after delivery, higher levels of free BPA $(0.80,3.07$, and $3.29 \mathrm{ng} / \mathrm{mL})$ were detectedcompared to the donors breast feeding for over 1 month. The very small number of samples limits the possibility of generalising the obtained results and the difference in the protein/fat composition between colostrum and mature breast milk limit the possibility of comparing the two groups. In addition, since no information is available on possible treatment with medical devices during the hospitalization period of the three mothers, a parenteral exposure to BPA cannot be excluded. This information could be useful for understanding the influence of medical devices on the exposure of other groups at risk, like the infants.

In two studies, ELISA (Kuruto-Niwa, 2007) or HPLC-FLD (Sun et al., 2004) methods were used to detect total BPA in breast milk: $1.4-7.1 \mathrm{ng} / \mathrm{mL}$; median $=3.0 \mathrm{ng} / \mathrm{mL}$ in samples from 101 healthy mothers in the former and $0.28-0.97 \mathrm{ng} / \mathrm{mL}$ in 23 samples in the latter. However, the reliability of the methods is unknown and therefore, limits the relevance of these data. In two studies in the USA, free and total BPA in breast-milk samples were quantified by isotope-dilution HPLC-MS/MS (Ye et al, 2006; 2008). Free BPA was detected in $60 \%$ of the 20 analysed samples with a median of $0.4 \mathrm{ng} / \mathrm{mL}$ (below the LOQ) and a maximum of $6.3 \mathrm{ng} / \mathrm{mL}$ (Ye et al, 2006), with free BPA being around $36 \%$ of total BPA. In their later study, Ye et al. (2008) additionally analysed 4 milk samples. The free and total BPA concentrations were $0.41-1.54 \mathrm{ng} / \mathrm{mL}$ and $0.73-1.62$ $\mathrm{ng} / \mathrm{mL}$, respectively, with free BPA in the individual samples accounting for $50-99 \%$ of the total. The presence of $\beta$-glucuronidase in human milk (Gaffney et al., 1986, Grazioso and Buescher, 1996) could be the cause for high free BPA. However, importantly, the glucuronide could be deconjugated in infants' guts by intestinal $\beta$-glucuronidases of bacterial origin, but not in neonates because of the low levels of intestinal flora, which usually develop at the $8^{\text {th }}$ postnatal month.

Otaka et al. (2003) reported free BPA content of 0.65 and $0.70 \mathrm{ng} / \mathrm{mL}$ in 2 out of 3 human milk samples by using a GC-MS method, whereas in a more recent study (Yi et al, 2010), free and total BPA in milk samples from 100 mothers (within two weeks from delivery) were detected by LC-MS/MS at values higher than $10 \mathrm{ng} / \mathrm{mL}$. 
On the basis of available biomonitoring and exposure data, exposure to BPA from nonfood sources was hypothesised to be potentially relevant (Calafat et al., 2009; Stahlhut et al., 2009; Taylor et al., 2011), but is generally lower than exposure from food by at least one order of magnitude for most studied subgroups (Geens et al, 2012). Dietary exposure was estimated to contribute for more than $90 \%$ of the overall BPA-exposure for non-occupationally exposed individuals (Geens et al., 2012) and exposure through dust ingestion, dental surgery and dermal absorption from thermal paper accounted for less than $5 \%$. As a consequence, total BPA blood concentrations should be directly related to contaminated food consumption, and individuals with long fasting times before urine collection should have substantially lower BPA urine levels than those with shorter fasting times. The study design and sample collection are again highlighted as a crucial issue. A specific case remains exposure to BPA through release from medical devices, especially in some subgroups as dialysis patients and infants in Intensive Care Units.

However, considering the toxicokinetics profile of BPA, any significant degree of bioaccumulation is not expected. If, following repeated exposure, BPA accumulation occurs in slowly releasing tissues, such as fat, then BPA concentrations in urine should increase with age. This is not the case in humans: individuals in the range 60-85 years old had lower levels than younger people enrolled in the study of Stahlhut et al. (2009).

Recently, the French Agency for Food, Environmental and Occupational Health \& Safety (ANSES, Paris, France) published their report on the evaluation of the risk of BPA for human health (ANSES, 2013). The report provides information on the aggregated exposure of pregnant women and unborn children due to air, dust and food in France. The median internal exposure was $1.68 \mathrm{ng} / \mathrm{kg}$ per day, whereas the maximum internal exposure of all three exposure routes ( $95^{\text {th }}$ percentile) was estimated at $4.18 \mathrm{ng} / \mathrm{kg}$ per day of which $84 \%$ was due to food, $4 \%$ due to dust and $12 \%$ due to air exposure (ANSES, 2013). The conversion factor used from external to internal exposure was $0.03(3 \%)$. In this evaluation, ANSES excluded possible exposure to medical devices including dental materials, and cosmetics. Similar to EFSA, ANSES has also concluded that the diet is the main source of BPA exposure for the general population.

EFSA (2013) estimated the BPA exposure due to dietary uptake of BPA. The highest exposure for children older than 6 months and up to 10 years of age was $857 \mathrm{ng} / \mathrm{kg}$ b.w./day and for infants days 1-5 after birth $495 \mathrm{ng} / \mathrm{kg}$ b.w/day. For adults, the highest exposure of $388 \mathrm{ng} / \mathrm{kg}$ b.w./day was estimated.

In a recent overview, the release of BPA from PC baby bottles into food and food simulants was evaluated (Hoekstra and Simoneau 2013). For the various foods and food simulants including oil simulants, ethanol, $3 \%$ acetic acid and water, the release varied between $<0.00005 \mathrm{mg} / \mathrm{L}$ to $5.9 \mathrm{mg} / \mathrm{L}$. In assays performed according to EU regulations, considering the temperature of food in baby bottles, all values observed were below the regulatory limit (Hoekstra and Simoneau 2013).

\subsubsection{Non-oral exposure routes}

Most studies and evaluations have focused on the potential for BPA exposure from dietary sources. BPA exposure from other routes, namely through dermal and inhalation routes, have received far less attention. Few studies have estimated total BPA exposure from multiple sources. Data from available studies on BPA exposure from dermal and inhalation exposure routes are described below.

EFSA estimated the contribution of non-oral exposure to the total exposure to BPA (EFSA 2015). For adolescents and adults, the exposure to thermal paper was estimated to be two to three times higher than the exposure to oral dietary BPA when the dermal exposure to thermal paper was converted to an equivalent oral dose. Although dust and cosmetics were also considered, their contribution to the BPA exposure was limited (up to 
$1.5-2 \%$ of the oral exposure for dust, and $2-9 \%$ of the oral exposure for cosmetics). For the total exposure to non-dietary sources cosmetics had the highest contribution being approximately $3-4 \%$ of the total non-dietary exposure as estimated by EFSA.

\section{BPA in Cosmetics and other consumer products}

Zhu et al. (2010) employed molecularly imprinted layer-coated silica nanoparticles for extraction of BPA from 3 cosmetic samples followed by determination by HPLCfluorescence detection: Shampoo $1.71 \mathrm{nmol} / \mathrm{g}(398 \mathrm{ng} / \mathrm{g})$; bath lotion $2.69 \mathrm{nmol} / \mathrm{g}(614$ $\mathrm{ng} / \mathrm{g})$; cosmetic cream $1.46 \mathrm{nmol} / \mathrm{g}(333 \mathrm{ng} / \mathrm{g})$. Cacho et al. (2013) determined BPA in 30 personal care products by extraction using ethylene glycol-silicone coated stir bars followed by thermal desorption-gas chromatography-mass spectrometry. BPA was found in six of the thirty analyzed samples (shower gel, hair gel, face cream, make-up remover and mouthwash) at concentrations ranging from $30.9 \mathrm{ng} / \mathrm{g}$ to $88.3 \mathrm{ng} / \mathrm{g}$. Employing GCMS in full scan mode, Dodson et al. (2012) determined up to 55 substances, including BPA, in 298 consumer products. BPA at concentrations $1-100 \mu \mathrm{g} / \mathrm{g}$ was found in 15 samples, including a vinyl shower curtain and pillow protector, dish and laundry detergent, tub and tile cleaner, soaps, lotions, shampoo, conditioner, shaving cream, nail polish, and sunscreen. However, uncertainty in BPA determination in consumer products performed by GC-MS in full scan mode may be very high. A large variation in BPA levels found in cosmetic products in the three studies may be associated with the uncertainty of measurements by three different methods. It may not be relevant to evaluate BPA exposure from cosmetics at present due to few and non-comparable data. However, in exaggerated exposure estimation, EFSA (2013) concluded that the contribution for BPA exposure due to cosmetics only contributes less than $3 \%$ of the total BPA exposure.

\section{Dermal exposure}

A common source of BPA exposure to consumers may be due to its presence in various types of papers. The amount of BPA used in thermal paper in the EU is 1,700 tons (EC, 2008). Of 13 thermal printing papers analysed, 11 were found to contain 8-17 g BPA $/ \mathrm{kg}$ paper (Biedermann et al., 2010). Vinggaard et al. (2000) reported BPA concentration 0.6 - $24 \mathrm{mg} / \mathrm{kg}$ (mean $6.21 \mathrm{mg} / \mathrm{kg}$ ) in 9 kitchen paper towels/kitchen rolls made of 80 $100 \%$ recycled paper. In toilet papers, BPA was found to be $46.1 \mathrm{mg} / \mathrm{kg}$ (Gehring, 2004). Lopez-Espinosa (2007) found BPA (geometric mean $2.38 \mathrm{ng} / \mathrm{g}$ of material) in $47.50 \%$ of samples of paper and cardboard investigated independent of the percentage of recycled material and the composition of the paper.

Biedermann et al. (2010) found that when taking hold of a receipt consisting of thermal printing paper for $5 \mathrm{~s}$, roughly $1 \mu \mathrm{g}$ BPA $(0.2-6 \mu \mathrm{g})$ was transferred to the forefinger and the middle finger if the skin was rather dry and about ten times more if these fingers were wet or very greasy. Seventy-three percent of the BPA transferred to dry skin by holding thermal printer paper was extractable after $2 \mathrm{~h}$. The authors speculated that 27 $\%$ of the BPA transferred from the thermal printing paper to the finger was penetrated into the skin. From these data, exposure of a person repeatedly touching thermal printer paper for $8 \mathrm{~h} /$ day, such as at a cash register, could reach approximately $57 \mu \mathrm{g} / \mathrm{day}$. Sun et al. (2001) reported that BPA content in commercially available samples of PVC wrap film, PVC gloves and PVC hose to be $68 \pm 3.5 \mu \mathrm{g} / \mathrm{g}, 60.5 \pm 2.8 \mu \mathrm{g} / \mathrm{g}$ and $290.1 \mu \mathrm{g} / \mathrm{g}$, respectively. No data is available on BPA exposure from PVC gloves, but it cannot be ruled out that small amounts of BPA may be transferred to the skin when these gloves are used. BPA was reported in five types of plasticised PVC food wrapping films at 0,43 , 96, 98 and $483 \mathrm{mg} / \mathrm{kg}$ (Lopez-Cervantes and Paseiro-Losada, 2003). It is not known if these samples, purchased in Spain, were made in the EU or were imported or if they were produced before or after the December 2001 cessation date given by The European Council of Vinyl Manufacturers (KEMI, 2011). No more recent survey data on testing PVC for BPA could be found in the literature.

In the EU risk assessment report on BPA (ECB 2003), some worst-case scenarios for dermal exposure of BPA were described. For epoxy-based surface coatings and 
adhesives, the main route of exposure is dermal. Use of BPA in printing inks is considered to result in negligible exposure.

Exposure from brush application of antifouling paint (without protective clothing), based on a paint containing $40 \%$ epoxy-resin and a residual level of $10 \mathrm{ppm}$ BPA in the resin, will result in a dermal exposure up to $29 \mu \mathrm{g}$ BPA. The use of wood fillers without gloves will result in $9 \mu \mathrm{g}$ dermal exposure of BPA per event. Application of wood varnish without use of gloves will result in $3.6 \mathrm{\mu g}$ dermal exposure of BPA. Based on residual level of 1 ppm BPA in an adhesive, dermal exposure to BPA arising from the use of adhesives was calculated to be $0.014 \mathrm{mg}$ per event.

\section{Air and Dust}

Air and dust levels of BPA serve as another potential source for human BPA exposure. Staples et al. (1998) estimated $1000 \mathrm{t} /$ year release of BPA from industrial production. Fu and Kawamura (2010) found that the concentrations of BPA $\left(1-17,400 \mathrm{pg} / \mathrm{m}^{3}\right)$ ranged over 4 orders of magnitude in the world with a declining trend from the continent (except for the Antarctica) to remote sites. Matsumoto et al. (2005) found that BPA concentrations in urban ambient outdoor air in Osaka during six months ranged from 0.02 to $1.92 \mathrm{ng} / \mathrm{m}^{3}$, with increasing levels from autumn to winter and decreasing levels from winter to spring.

Wilson et al. (2007) found that potential sources of BPA exposure in preschool children included outdoor and indoor air and house dust, as well as soil from homes and daycare centers. Concentrations in indoor air from homes and daycare centers ranged from <LOD to 193 and $8.99 \mathrm{ng} / \mathrm{m}^{3}$, respectively. Concentrations in outdoor air ranged from <LOD to 44.6 and $51.5 \mathrm{ng} / \mathrm{m}^{3}$ in homes and daycare centers, respectively. Concentrations in dust ranged from <LOD to 707 and $156 \mathrm{ng} / \mathrm{g}$ in homes and daycare centers, respectively. Rudel et al. (2003) found BPA present in $86 \%$ of house dust samples at concentrations ranging from 0.2 to $17.6 \mu \mathrm{g} / \mathrm{g}$. Völkel et al. (2008) reported median BPA concentration in dust from 12 private houses in Germany to be $553 \mathrm{ng} / \mathrm{g}$ (range 117 to $1486 \mathrm{ng} / \mathrm{kg}$ ).

Geens et al. (2009) found that BPA concentration in indoor dust samples from 18 Belgian homes was 535-9730 ng/g; and dust samples from 2 offices contained 4685 and $8380 \mathrm{ng}$ BPA/g dust. Geens et al. (2009) calculated $29 \mathrm{ng} / \mathrm{d}$ (or $0.4 \mathrm{ng} / \mathrm{kg}$ b.w./day) BPA intake through dust by an adult with an average dust intake of $20 \mathrm{mg} / \mathrm{d}$ and a median BPA content of $1460 \mathrm{ng} / \mathrm{g}$. Exposure assessments, based on the different sources of BPA exposure from the National Toxicology Program, gave a range of $0.043-14.7 \mu \mathrm{g} / \mathrm{kg}$ b.w./day BPA for 1.5 and 6 year-old children and between 0.008 and $1.5 \mu \mathrm{g} / \mathrm{kg} \mathrm{b.w./day}$ for adults (US National Toxicology Program, 2008). Loganathan and Kannan (2011) measured BPA in dust from 50 homes and 6 laboratories in the Eastern USA. Mean and median concentrations of BPA in dust were 843 and $422 \mathrm{ng} / \mathrm{g}$, respectively. The authors calculated that the median daily intake of BPA via dust for adults and toddlers were 0.35 and $5.63 \mathrm{ng} / \mathrm{kg}$ b.w./day, respectively. The contribution of dust to total human intake of BPA was estimated to be $<1 \%$. Thus, despite the high concentrations of BPA measured in dust, this route of exposure seems to be a minor contributor to the total exposure.

According to EU risk assessment, BPA exposure as a result of brush application of antifouling paints (containing $40 \%$ epoxy-resin and a residual level of $10 \mathrm{ppm}$ BPA in the resin), in a worst-case scenario, resulted in inhalation exposure of $3 \times 10^{-4} \mu \mathrm{g}$ (ECB 2003). For wood varnish, a value of $0.02 \mu \mathrm{g}$ for inhalation exposure to BPA per event was calculated, in a worst-case scenario (ECB 2003).

Recently ANSES estimated that dust represented $4 \%$ of the total BPA exposure for pregnant women (ANSES, 2013). 


\subsubsection{Exposure to BPA from medical devices}

The major source of BPA exposure by medical devices is due to medical devices made from PC and PSU. With regard to the possible presence of BPA in medical devices made of PVC, notwithstanding the statement that the use of BPA for polymerization and as a stabiliser for storage of vinyl chloride monomer was discontinued in Europe from December 2001 (KEMI 2011). While European PVC manufacturers informed the SCENIHR that they no longer use BPA in PVC production, BPA-containing medical devices from extra-EU manufacturers may be available in Europe.

Only limited information on BPA exposure from medical devices was found in the scientific literature. Elevated blood levels of BPA in dialysis patients have been reported (Murakami et al., 2007; Krieter et al., 2009; Shintani and Hayashi, 2011). Calafat et al. (2009) found that total BPA (including BPA metabolites) geometric mean urinary concentration $(30.3 \mathrm{\mu g} / \mathrm{L})$ among prematurely born infants undergoing intensive therapeutic medical interventions was about ten times higher than that among the general population.

\section{Dialyzers}

Shintani (2001) reported average levels of BPA $(n=4)$ of 0.2 and $0.7 \mathrm{ppb}(\mathrm{ng} / \mathrm{mL})$ in the blood of patients exposed for $4 \mathrm{~h}$ to dialyzers (PSU fibers, PC housing) sterilized by autoclaving. The average concentrations in the blood of patients treated with two other dialyzers (both with PSU fiber, one with PC housing and other with polystyrene-butadiene copolymer housing) sterilized with gamma radiation, were below limit of detection $(0.02$ $\mathrm{ppb}$ or $\mathrm{ng} / \mathrm{mL}$ ). HPLC-MS was used for BPA detection. Samples (ca. $10 \mathrm{~mL}$ ) were collected before dialysis and after $4 \mathrm{~h}$ of dialysis treatment. Tests were also performed using saline solutions (according to ISO 10993-7) to compare with migration into blood, using the same dialyzer sterilized by the same procedure. BPA levels found were $0.1-0.2$ $\mathrm{ppb}(\mathrm{ng} / \mathrm{mL})$ in the saline solution and 0.2 to $0.7 \mathrm{ppb}(\mathrm{ng} / \mathrm{mL})$ for blood. Pre-treatment BPA blood levels were below the detection limit ( $0.02 \mathrm{ppb}$ in plasma). It was not stated in the publication if total or free BPA was measured and whether the blood was drawn from the patient or directly from the equipment.

Sajiki et al. (2008) reported levels of BPA in plasma of 53 hemodialysis patients, before and after hemodialysis, and in plasma of five healthy volunteers. The BPA measurements were performed by three different methods: LC/ECD, LC/MS and ELISA. The plasma BPA levels in 5 healthy persons were $0.033 \pm 0.075 \mathrm{ng} / \mathrm{ml}(L C / E C D)$ or $0.284 \pm 0.146 \mathrm{ng} / \mathrm{mL}$ (ELISA). Plasma BPA levels in patients before and after hemodialysis were $0.284 \pm 0.748$ $\mathrm{ng} / \mathrm{mL}$ and $0.642 \pm 1.443 \mathrm{ng} / \mathrm{mL}$ respectively when measured by $L C / E C D$, or 0.505 $\pm 2.125 \mathrm{ng} / \mathrm{mL}$ and $0.197 \pm 0.248 \mathrm{ng} / \mathrm{mL}$ respectively when measured by ELISA, or $0.310 \pm 0.840$ and $0.179 \pm 0.263 \mathrm{ng} / \mathrm{mL}$ respectively when measured by LC/MS. Individual values or range of BPA in plasma are not reported. The results of the study cannot be considered reliable because the recovery of $100 \mathrm{ng} / \mathrm{ml}$ BPA in plasma or water was only $61-72 \%$ by LC/MS and ELISA methods and the selectivity of LC/ECD method was very poor. In addition, the hemodialysis set-up used for any of the patientswas not described.

Murakami et al. (2007) determined the amount of BPA leaching from dialyzers with PSU membranes in the blood of 15 patients. An indication for an increase in BPA was found from $4.83 \pm 1.94 \mathrm{ng} / \mathrm{mL}$ blood prior to dialysis to $6.62 \pm 3.09 \mathrm{ng} / \mathrm{mL}$ thereafter (increase of $1.79 \mathrm{ng} / \mathrm{mL}$ ); however, a later second test with the same patients and the same dialyzers resulted in a much lower increase in BPA serum levels, from $3.78 \pm 2.57 \mathrm{ng} / \mathrm{ml}$ to $4.27 \pm 2.98(0.49 \mathrm{ng} / \mathrm{ml}$ increase). Both the quoted differences are not statistically significant; in addition, BPA concentration was measured by ELISA, which may be prone to cross reactivity with other phenols, leading to overestimation of BPA content.

Krieter et al. (2013) found that despite differences in BPA elution into water from 3 different types of dialyzers with high and low-flux PSU and polyethersulfone membranes, the plasma levels of 18 patients with chronic kidney disease treated with any of the 
dialyzers did not significantly change after a 4-week treatment period. On the other hand, BPA pre-dialysis plasma concentrations in patients were significantly higher (range $9.1 \pm 4.5-12.0 \pm 6.0 \mathrm{ng} / \mathrm{mL})$ than those found in the healthy control group $(n=24, \leq$ $0.2 \pm 0.1 \mathrm{ng} / \mathrm{mL}$ ). Fractions of protein-bound and free plasma BPA were found to have similar values in dialysis patients $(74 \pm 5 \%)$ and in the control group (70 $\pm 3 \%)$. ELISA method was used for BPA measurements.

\section{Dental materials}

Recent reports from different authorities addressing the risk assessment of BPA from various sources, especially food contact materials, have to some extent also addressed dental materials. The general conclusion is that the contribution from dental materials to the total exposure is low (Beronius and Hanberg, 2011; FAO/WHO, 2011; Environment Canada/Health Canada 2008;EC 2010b; US NTP-CERHR, 2008; EFSA, 2013). A report recently published by the Swedish National Board of Health and Welfare (Socialstyrelsen 2012) addressed "Bisphenol A in dental materials". The report summarizes research on in vitro and in vivo studies related to BPA from dental materials, and concludes that there is a possibility of low-dose exposure to BPA from dental materials, either as a contaminant (low amounts) or from degradation of Bis-DMA. In the report, calculations based on the maximum values of BPA found in fissure sealants and in composite materials, in combination with the actual amount of material used in clinical practice and a median 4year life-time of a composite restoration, suggest a maximum exposure of $0.06 \mu \mathrm{g}$ $\mathrm{BPA} /$ day from fissure sealants, and a maximum exposure of $0.36 \mu \mathrm{g}$ BPA/day from composite restorations.

Kang et al. (2011) assessed the changes in BPA levels in saliva and urine after placing lingual bonded retainers. Liquid chromatography/mass spectrometry was used to examine the BPA levels in the saliva and urine samples collected from 22 volunteers who received a lingual bonded retainer on their mandibular dentition. Samples were collected immediately before placement and 30 minutes, 1 day, 1 week, and 1 month after placement. The salivary BPA level was detected in the samples collected just after placement with a mean concentration of $5 \mathrm{ng} / \mathrm{mL}$ and a maximum of $21 \mathrm{ng} / \mathrm{mL}$.

Olea and co-workers (1996) recruited 18 healthy men and women who were treated with one molar sealant, and found no composite components before treatment (except in one subject who was excluded), whereas after treatment all saliva samples contained variable amounts of BPA ranging from 90 to $931 \mu \mathrm{g}$ in the total saliva produced by the volunteers, who spit and collected their saliva in glass vials throughout the whole 60-minute period.

Arenholt-Bindslev and colleagues (1999) enrolled eight adult male volunteers who were treated with four molar sealants of two different brands. BPA assessment was done before, immediately after, 1 hour after and $24 \mathrm{~h}$ after sealant placement. Before treatment, there was no detectable BPA, but after sealant placement BPA levels ranged between $0.3-2.8 \mathrm{ppm}(\mu \mathrm{g} / \mathrm{mL})$. For materials containing BisDMA, the BPA was found to be released immediately into the saliva after application, while at $24 \mathrm{~h}$ after the placement BPA levels in saliva were back to the pretreatment levels (Schmalz et al., 1999; Arenholt-Bindslev et al., 1999).

Fung et al. (2000) recruited 40 adults and found BPA in some saliva specimens collected at 1 hour and $3 \mathrm{~h}$ after treatment, ranging from 5.8 to $105.6 \mathrm{ppb}(\mathrm{ng} / \mathrm{mL})$. In addition, Joskow and co-workers (2006), using two brands of sealants, assessed saliva samples in 14 patients who received a mean number of six sealants. Saliva samples were collected pretreatment, immediately after, and 1-hour post treatment. Mean BPA before treatment that was $0.30 \mathrm{ng} / \mathrm{mL}$ reached $42.8 \mathrm{ng} / \mathrm{mL}$, immediately after placement.

Zimmerman-Downs (2010) enrolled 30 adults who were treated with one or four occlusal sealants. BPA assessment was done 1 hour prior and 1,3 , and $24 \mathrm{~h}$ post sealant placement. BPA was elevated significantly from baseline measurements before sealant (range 0.07 to $6.00 \mathrm{ng} / \mathrm{mL}$ ) at all post-treatment time points for both groups, with main peak amounts of 3.98 and $9.08 \mathrm{ng} / \mathrm{mL}$ for the one-occlusal sealant group and the four occlusal-sealants group, respectively. 
More recently, Han et al. (2012) made a survey including 62 children serving as control, without restorations in the oral cavities, and 62 children treated with more than 4 sealants. A possible relationship was found: BPA level of control was $0.40 \mu \mathrm{g} / \mathrm{L}$, whereas BPA levels in saliva of treated children was $0.92 \mu \mathrm{g} / \mathrm{L}$ after controlling for confounders.

Kingman and co-workers (2012) collected saliva before and after ( $<1,1-8$ and 9-30 h) from 172 participants receiving composite restorations. BPA concentrations in saliva significantly increased within an hour after treatment from 0.43 to $0.64 \mathrm{ng} / \mathrm{mL}$ (Geometric Mean), together with other restorations materials. For the period between 9$30 \mathrm{~h}$, an increase was observed in the urine (Kingman et al., 2012). The increase was from $1.77 \mathrm{ng} / \mathrm{mL}$ to $2.59 \mathrm{ng} / \mathrm{mL}$ in females $(p=0.005)$, and from $1.55 \mathrm{ng} / \mathrm{mL}$ to 2.16 $\mathrm{ng} / \mathrm{mL}$ in males $(\mathrm{p}=0.25)$. Later time points were not investigated.

In summary, the release of BPA into the saliva occurs in patients receiving dental restorations. Placement of resin-based composite restorations polymerised in situ was associated with detectable increases in saliva of BPA. The release mainly occurs during the few $h$ directly after application and contributes to the oral exposure to BPA. An overview of the BPA releases, as discussed above for medical devices used in dentistry, are presented in Table 2 below.

Recently, Kloukas et al. (2013) made a systematic review of in vivo BPA release from dental pit and fissure sealants. Six interventional and two observational studies, examining in vivo BPA release in human salivary, blood and urinary samples, were included. BPA levels identified in saliva ranged from traces below the method's detection limit to $30 \mu \mathrm{g} / \mathrm{mL}$. In urine, BPA quantities spanned from $0.17 \mathrm{mg} / \mathrm{g}$ to $45.4 \mathrm{mg} / \mathrm{g}$. The quantitative analysis showed evidence of BPA release one hour after sealant placement compared to the amount traced before restoration (Stouffer's $z$ trend: $<0.001$ ).

From the qualititative and quantitative synthesis of available studies, it is reasonable to conclude that some BPA is released after placement of dental pit and fissure sealants in the oral cavity. The largest quantities are detected in saliva immediately after or one hour after their placement.

Table 2: BPA in saliva after application of a dental sealant

\begin{tabular}{|c|c|c|c|c|}
\hline Reference & $\begin{array}{l}\text { Number/ } \\
\text { I ndividuals }\end{array}$ & Time & $\begin{array}{c}\text { BPA } \\
\text { concentration }\end{array}$ & $\begin{array}{l}\text { Maximum } \\
\text { BPA reported }\end{array}$ \\
\hline Kingman et al., 2012 & 150/adults & At $<1 \mathrm{~h}$ & $0.64 \mathrm{ng} / \mathrm{mL}$ & \\
\hline Han et al., 2012 & $\begin{array}{l}\text { 124/ } \\
\text { Children }\end{array}$ & & $0.92 \mathrm{ng} / \mathrm{mL}$ & $8.305 \mu \mathrm{g} / \mathrm{L}$ \\
\hline $\begin{array}{l}\text { Zimmerman-Dawns et } \\
\text { al., } 2010\end{array}$ & 30/adults & At $1 \mathrm{~h}$ & $9.08 \mathrm{ng} / \mathrm{mL}$ & \\
\hline Joskow et al., 2006 & 14/adults & At $1 \mathrm{~h}$ & $42.8 \mathrm{ng} / \mathrm{mL}$ & $96.2 \mathrm{ng} / \mathrm{ml}$ \\
\hline Sasaki et al., 2005 & 21/adults & & & $100 \mathrm{ng} / \mathrm{ml}$ \\
\hline Fung et al., 2000 & 40 adults & At $1 \mathrm{~h}$ & & $\begin{array}{ll}105.6 & \mathrm{ppb} \\
(\mathrm{ng} / \mathrm{mL})\end{array}$ \\
\hline $\begin{array}{l}\text { Arenholt-Bindslev et al., } \\
1999\end{array}$ & $\begin{array}{l}8 \\
\text { volunteers }\end{array}$ & $\begin{array}{l}\text { Immediately } \\
\text { after }\end{array}$ & & $\begin{array}{ll}2.8 & \mathrm{ppm} \\
(\mu \mathrm{g} / \mathrm{mL}) & \end{array}$ \\
\hline Olea et al., 1996 & 28/adults & $\begin{array}{l}\text { All the saliva } \\
\text { produced in } \\
1 \mathrm{~h}\end{array}$ & & $931 \mu \mathrm{g}$ \\
\hline
\end{tabular}

Note to the table: The studies are listed in the chronological order (most recent first). 
Only one study was identified in the category of orthodontic adhesives releasing BPA in saliva. Kang and coworkers (2011) evaluated the changes of BPA levels in saliva before and after placing a lingual bonded retainer on the mandibular dentitions of 2 volunteers. Samples were obtained immediately before placement of the retainer and $30 \mathrm{~min}, 1$ week and 1 month after placement. Mean salivary levels of BPA were $5.04 \mathrm{ng} / \mathrm{mL}$ (range 0.85 $20.88 \mathrm{ng} / \mathrm{mL}$ ) in the immediately collected samples.

\section{Medical procedures}

A study by Sakurai (2002, only abstract available in English) indicates migration of BPA from cardiopulmonary bypass (CPB) circuits during open=heart surgery and when using a saline priming solution. Blood samples were obtained from six patients who underwent open-heart surgery after the CPB process was initiated and at the termination. For the priming solution study, eight circuits were used and as control three samples were collected directly from the saline in a polyethylene container. BPA levels measured in the blood were $0.3 \pm 0.2 \mu \mathrm{g} / \mathrm{L}$, after the commencement of CBP and $0.4 \pm 0.3 \mu \mathrm{g} / \mathrm{L}$ when it was finished. In the priming solution from the circuits, higher levels were found: $0.9 \pm 1.1$ $\mu \mathrm{g} / \mathrm{L}$. No BPA was detected in the control samples. BPA was thought to be leached from the circuit because parts of the reservoir and of the oxygenator were made of PC containing BPA.

Calafat et al. (2009) found that among prematurely born infants undergoing intensive therapeutic medical interventions, the total BPA (including BPA metabolites) geometric mean urinary concentration was $30.3 \mu \mathrm{g} / \mathrm{L}$ with $946 \mu \mathrm{g} / \mathrm{L}$ as the highest value measured that was about ten times higher than that among children 6-11 year old (Calafat et al., 2008). More than $90 \%$ of the BPA detected in the urine of the prematurely born infants was in its conjugated (e.g. glucuronide, sulfate) form. The authors attributed the high BPA levels to the recent treatment given to the infants after birth rather than to the in utero exposure. Notably, BPA total concentrations among infants in one health care unit were about 17 times higher than those among the infants hospitalized in the second one. The authors suggested that this difference may be due to parenteral exposure via medical devices used, which is supported for the same neonates by a strong association between di(2-ethylhexyl) phthalate (DEHP) and BPA total concentrations.

Based on the iso-propanol extraction of BPA from PC pellets $(0.2-0.3 \mathrm{mg} / \mathrm{kg}$ ) and assuming that $100 \%$ of this BPA would also leach from the material during clinical use within a single day, the patient exposure could be estimated as high as $0.0386 \mu \mathrm{g} / \mathrm{kg}$ b.w./day, considering a body weight of $58 \mathrm{~kg}$. This estimation was presented in the data submitted through the Call for Information.

Women who had caesarean sections showed much higher levels of free and total urinary BPA than women giving birth naturally, the $95^{\text {th }}$ percentile being $273.9 \mu \mathrm{g} / \mathrm{L}$ vs. $4.2 \mu \mathrm{g} / \mathrm{L}$, with data adjusted for creatinine levels (Vandentorren et al., 2011). However, as contamination with BPA from exogenous sources during sample storage can be excluded, the high level of BPA was attributed to the release of BPA from the urinary catheter used for collection. Indeed, supplemental studies on the release of BPA from catheterization probes into urine at room temperature for 12 and $24 \mathrm{~h}$ clearly indicate the time dependence of free BPA extraction from the probes up to approximately $300 \mu \mathrm{g} / \mathrm{L}$.

\section{Conclusion}

BPA release was demonstrated from dialyzers, dental materials, circulation equipment, neonatal care medical devices, and urinary catheters. Some clinical studies involving hemodialyzers indicated that there were no significant BPA plasma level changes after dialysis treatment. However, one study reported a difference in BPA plasma levels between dialysis patients and controls. The release of BPA from other medical device is generally very poorly characterised in human studies.

Dental materials release BPA, especially shortly $(0-1 \mathrm{~h})$ after placement. The levels of BPA detected in saliva ranged from $0.64 \mathrm{ng} / \mathrm{mL}$ to $30 \mu \mathrm{g} / \mathrm{mL}$. This wide range in BPA measurements may indicate a continuous reduced leaching of BPA from dental materials, 
probably due to the reduced use of bis-DMA, as well as reflect variations in the analytical methodology used.

\subsubsection{Exposure to BPA from medical devices under different scenarios}

Scenarios in hospitals

Taking into account the many possible sources of exposure of patients during hospital care and the scarcity of information related to release of BPA from medical devices, six scenarios were considered to be representative situations.

(i) External contact with a medical device containing BPA;

(ii) Contact with oral/dental material and / or orthodontic equipment;

(iii) Contact with implants such as valve, pacemaker, insulin dispenser made in PC;

(iv) Hemodialysis;

(v) Prolonged surgical procedures such as bypass operations and transplantations;

(vi) Prolonged exposure to different sources of BPA in intensive care units.

The specific case of health care workers using PVC gloves which may contain traces of BPA (around $0.05 \%$ ) was not considered even through a few cases were reported describing contact dermatitis against BPA (Aalto-Korte et al., 2003). European PVC manufacturers have stopped using BPA in PVC products (KEMI, 2011). However, PVC as a possible source of BPA exposure cannot be completely excluded because BPAcontaining PVC may still be used in the EU due to medical devices, including gloves, coming from outside of the EU because of the global market for medical devices.

\section{(i) External contact with a medical device-containing BPA.}

This situation occurs very frequently because many medical devices such as catheters, trocars, laparoscopic and endoscopic instruments, breast pumps, prescription spectacles and lenses, etc., may contain residues of BPA (see also Annex I). In this scenario, exposure may be repeated but, taking into account the small surface area of the medical device in contact with skin or mucosa, the amount of released BPA is low. Taking the example of a disposable laryngoscope blade made of PC, the surface area in contact depends on the size and the shape. Sizes range from 00 (premature) up to 3 (large, adult). The surface area is estimated to be in the range ca. 5 to $25 \mathrm{~cm}^{2}$. For a $1 \mathrm{~kg}$ premature infant and a $60 \mathrm{~kg}$ adult this is $5 / 1=5$ and $25 / 60=0.4 \mathrm{~cm}^{2} / \mathrm{kg} \mathrm{b} . \mathrm{w}$. The application is single use. The contact medium is mucosa, the temperature is $37^{\circ} \mathrm{C}$ and the contact duration is a few minutes at most.

Calculation: For the release of BPA from PC, EFSA (2013) considered tableware (plates, cups, etc.) made of PC and using from the literature the migration data into water, $3 \%$ acetic acid and $50 \%$ ethanol under testing conditions of $2 \mathrm{~h}$ at $70^{\circ} \mathrm{C}$. These data were combined with data from the EFSA call for data obtained under the same testing conditions. An upper-bound value of $0.0013 \mathrm{mg} / \mathrm{L}$ was derived from the 2 hour contact times. Rounding this value up to $0.002 \mathrm{mg} / \mathrm{kg}$ and assuming a conservative surface area: volume ratio of $1000 \mathrm{~cm}^{2} / \mathrm{L}$, migration of BPA from PC articles equates to $2 \mathrm{ng} / \mathrm{cm}^{2}$. Assuming (conservatively) that each contact use of a laryngoscope releases the same amount (on an area-related basis) as PC for $2 \mathrm{~h}$ of contact at $70^{\circ} \mathrm{C}$, exposure from the 
laryngoscope would be $1 \mathrm{ng} / \mathrm{kg}$ b.w./use for premature infants and $0.08 \mathrm{ng} / \mathrm{kg} \mathrm{b.w./use}$ for adults.

\section{(ii) Contact with dental material and orthodontic equipment}

Dental materials may be divided into two scenarios

- Short-term exposure in conjunction with dental treatment: one full crown restoration of a molar may release after $24 \mathrm{~h}$ on average $57.38 \mathrm{nmol}(13 \mu \mathrm{g})($ Van Landuyt, 2011)

- Long-term exposure from the use of dental materials: After $24 \mathrm{~h}$, no elevation in BPA-level in saliva is found (Kingman et al., 2012; Kang et al., 2011). For the period between 9-30 $\mathrm{h}$ an increase was observed in the urine (Kingman et al., 2012). The increase was from $1.77 \mathrm{ng} / \mathrm{mL}$ to $2.59 \mathrm{ng} / \mathrm{mL}$ in females $(p=0.005)$, and from $1.55 \mathrm{ng} / \mathrm{mL}$ to $2.16 \mathrm{ng} / \mathrm{mL}$ in males $(\mathrm{p}=0.25)$.

Additionally, there may be a possible exposure from the procedure of removing a restoration. This situation is not well covered in the scientific literature and may need further investigation.

Calculation: Given the considerable uncertainty with respect to the chemical composition of dental materials used, both now and in the past, the value of $13 \mu \mathrm{g}$ released in the one day following treatment (Van Landuyt, 2011) is considered for short-term (acute) exposure and this would be $200 \mathrm{ng} / \mathrm{kg} \mathrm{b.w./day} \mathrm{for} \mathrm{a} 60 \mathrm{~kg} \mathrm{b.w}$. adult. The value of 0.36 $\mu \mathrm{g} /$ day $(6 \mathrm{ng} / \mathrm{kg} / \mathrm{b} . \mathrm{w} . /$ day) from the Swedish review is considered for the long-term exposure scenario for adults undergoing composite restorations and $0.06 \mu \mathrm{g} / \mathrm{day}$ (2 $\mathrm{ng} / \mathrm{kw}$ b.w./day) for a $30 \mathrm{~kg}$ child treated with fissure sealants.

For orthodontic equipment, the case of PC brackets secured using adhesives is considered. Release of BPA from adhesives and PC brackets under experimental conditions is presented in table 1 above.

Calculation 1: For short-term exposure, there was BPA release of $66 \mu \mathrm{g} / \mathrm{L}$ from a lightcured adhesive resin analysed at day-7 (Sunitha et al., 2011). With a typical saliva production of $15 \mathrm{~mL} / \mathrm{kg}$ b.w./day, the short-term exposure would be $140 \mathrm{ng} / \mathrm{kg} \mathrm{b.w./day.}$

Calculation 2: For medium-term exposure, there was a BPA release of $2.9 \mu \mathrm{g} / \mathrm{L}$ from a light-cured adhesive analysed after 30 days immersion in simulant (Eliades et al., 2011). With a typical saliva production of $15 \mathrm{~mL} / \mathrm{kg} \mathrm{b.w./day,} \mathrm{medium-term} \mathrm{exposure} \mathrm{would} \mathrm{be}$ $1.5 \mathrm{ng} / \mathrm{kg} \mathrm{b.w./day.} \mathrm{Adding} \mathrm{this} \mathrm{to} \mathrm{the} \mathrm{daily} \mathrm{exposure} \mathrm{possible} \mathrm{from} \mathrm{the} \mathrm{PC} \mathrm{brackets} \mathrm{(see}$ below) gives exposure of 13.5 and $7.5 \mathrm{ng} / \mathrm{kg} \mathrm{b.w./day} \mathrm{for} \mathrm{a} 30 \mathrm{~kg} \mathrm{b.w}$. child and a $60 \mathrm{~kg}$ b.w. adult, respectively.

Calculation 3: For long-term exposure, Watanabe et al. (2001) found that PC brackets released BPA when immersed in water. The BPA increase in the water was 3.8-fold after 12 months immersion in water at $37^{\circ} \mathrm{C}$, and 14 -fold after 14 weeks immersion in water at $60^{\circ} \mathrm{C}$, when compared to virgin water values. BPA release started slow at $0.4 \mu \mathrm{g} / \mathrm{g}$ of PC until 6 months and increased to $3.8 \mu \mathrm{g} / \mathrm{g}$ of PC at 12 months immersion in water at $37^{\circ} \mathrm{C}$. With a typical weight of approximately $40 \mathrm{mg}$ and a maximum number of 24 applications, the total weight of brackets would be ca. $1 \mathrm{~g}$ of PC. The release would be 10 $\mathrm{ng} /$ day or an exposure of 0.33 and $0.17 \mathrm{ng} / \mathrm{kg} \mathrm{b.w./day} \mathrm{for} \mathrm{a} 30 \mathrm{~kg} \mathrm{b.w}$. child and a 60 $\mathrm{kg}$ b.w. adult, respectively. In this long-term scenario, it is assumed that any release of BPA from the adhesive used to secure the brackets has declined to a not detectable level.

\section{(iii) Contact with an implant}

According to the implanted device such as a valve, pacemaker or a dispenser made in PC, the surface in contact with tissues and body fluids may be estimated (worst case scenario) to be $50 \mathrm{~cm}^{2}$. EFSA (2013) considered the migration from PC baby bottle into food simulants and they derived an average migration of $0.89 \mu \mathrm{g} / \mathrm{L}$ and a high migration of $4.56 \mu \mathrm{g} / / \mathrm{L}$. The most usual test conditions employed with food simulants was 10 days 
contact at $40^{\circ} \mathrm{C}$. By rounding-up, taking the high migration of $0.5 \mu \mathrm{g} / \mathrm{L}$ per day and the conservative area volume ratio (see above) of $1000 \mathrm{~cm}^{2} / \mathrm{L}$, a release value of 0.5 $\mathrm{ng} / \mathrm{cm}^{2} /$ day can be derived.

Calculation 1: $50 \mathrm{~cm}^{2}$ releasing $0.5 \mathrm{ng} / \mathrm{cm}^{2} /$ day would give exposure of $25 \mathrm{ng}$ per day, this being $0.4 \mathrm{ng} / \mathrm{kg}$ b.w./day for a $60 \mathrm{~kg} \mathrm{b.w.} \mathrm{adult} \mathrm{or} 0.8 \mathrm{ng} / \mathrm{kg} \mathrm{b.w./day} \mathrm{for} \mathrm{a} 30 \mathrm{~kg}$ b.w. child. This release rate includes the possibility of hydrolysis of the PC surface rather than only classical diffusion-migration.

Calculation 2: Mass of $10 \mathrm{~g} \mathrm{PC}$ in the medical device and with up to $10 \mathrm{mg} / \mathrm{kg}$ residual BPA gives a residual content of $100 \mu \mathrm{g}$. If $10 \%$ were released in the first 30 days of use of the implant (highly unlikely) this would give $333 \mathrm{ng} /$ day or 6 and $11 \mathrm{ng} / \mathrm{kg} \mathrm{b.w./day}$ for a $60 \mathrm{~kg}$ adult or $30 \mathrm{~kg}$ child respectively. This calculation assumes that the PC does not hydrolyse in situ.

\section{(iv) Haemodialysis}

Patients experiencing renal failure need, before eventual kidney transplantation, regular blood separation performed by exchange between blood and osmolar dialysis fluid through a dialyser comprising a membrane and a support unit. The carter is often made by PC and hold PSU membranes (often hollow filters) which may release BPA as explained in the paragraphs 3.6.2 and 3.7.2.

The highest values of release of BPA by such dialysers, as found in the literature, are comprised between $2 \mu \mathrm{g} /$ module (Haishima et al., 2001) and $4 \mu \mathrm{g} / \mathrm{session}$ (Fink, 2008). According to their medical problems, weight, age and activity, the patients undergo 3 to 6 dialysis sessions each week (mean duration 3-4 h) for the most active patients (>70 kg + sport practice), down to 2 sessions a week for the aged inactive and $<50 \mathrm{~kg}$ patients.

Calculation: In the worst care scenario, $4 \mu \mathrm{g}$ of BPA per session, 6 times a week, for a 60 $\mathrm{kg} \mathrm{b.w.} \mathrm{adult} \mathrm{the} \mathrm{exposure} \mathrm{would} \mathrm{be} 57 \mathrm{ng} / \mathrm{kg} \mathrm{b.w./day.}$

\section{(v) Prolonged surgical procedures}

In this case of extracorporeal circulation (by-pass) or transplantation / implantation of an artificial organ such as heart, according to the size and the nature of catheters and artificial organ, the release of BPA may be estimated in the same range as in the care of hemodialysis. For infants and children, although the use of medical devices may be scaled-down, it is assumed that there is the same release of BPA but with a smaller body weight. Consequently the short-term exposure would be 685,114 , and $57 \mathrm{ng} / \mathrm{kg} / \mathrm{b}$.w. for an infant, child and adult of 5, 30 and $60 \mathrm{~kg}$ body weight, respectively.

\section{(vi) Prolonged exposure to different sources of BPA in intensive care units}

a) in adult intensive care units

Adult patients hospitalized in intensive care units (ICU) are treated with the use of:

- Venous or arterial catheters (inserted by peripheral way, PICC line), with a classical duration comprised between 2 - 3 weeks to some months oncohematology (Kabsy et al., 2010)

- Respiratory assistance using tubing (some hundred grams)

b) in neonatal intensive care units

The neonates' BPA exposure can be via the respiratory tract (incubator walls and respiratory tubing), the blood (catheters) and via the oral route (tubing, mother's milk collected via breast pump in PC). 
Neonates hospitalized in intensive care units (NICU) are treated with the same medical devices as adults, often for several months, like:

- Venous or arterial catheters

- Respiratory assistance

- Incubator (the walls are made in polycarbonate)

For an incubator hood of dimensions $80 \times 60 \times 70 \mathrm{~cm}(\mathrm{~W} \times \mathrm{D} \times \mathrm{H})$, the surface area of PC is $24400 \mathrm{~cm}^{2}$. In fact these dimensions are not used in the calculations, just the vapour pressure.

Calculation 1: "In hoods of neonatal incubators and neonatal intensive care units, BPA could not be detected (LOD approximately $50 \mu \mathrm{g} / \mathrm{m}^{3}$ )" (see paragraph 3.6.2, data submited via the call for information).

Breathing volume of a prematurely born infant ca. $100 \mathrm{~mL} / \mathrm{kg} / \mathrm{min}$ gives $0.144 \mathrm{~m}^{3} / \mathrm{kg}$ b.w./day. If the air contained BPA at the detection limit and assuming complete uptake out of the air, the exposure would be $\leq 7 \mu \mathrm{g} \mathrm{BPA} / \mathrm{kg} \mathrm{b.w./day.} \mathrm{Due} \mathrm{to} \mathrm{the} \mathrm{high} \mathrm{detection}$ limit, this calculation is likely to be unrealistic; therefore, a second calculation was performed.

Calculation 2: The vapour pressure of BPA is $5.3 \times 10^{-6} \mathrm{~Pa}$ at $25^{\circ} \mathrm{C}$. Assuming gas ideality, since 1 mole of a gas occupies $25 \mathrm{~L}$ at STP at this vapour pressure, then $25 \mathrm{~L}$ of air above pure BPA solid would contain $5.3 \times 10^{-11}$ moles or $1.2 \times 10^{-8} \mathrm{~g}$ of BPA.Correspondingly, one $\mathrm{m}^{3}$ would contain $4.8 \times 10^{-7} \mathrm{~g}$. Residual BPA in PC is up to 10 $\mathrm{mg} / \mathrm{kg}$ or a $10^{-5}$ mass fraction. Assuming for simplicity a linear concentration-vapour pressure relationship, BPA in air at equilibrium with PC would be ca. $4.8 \times 10^{-12} \mathrm{~g} / \mathrm{m}^{3}$. Exposure $=7 \times 10^{-13} \mathrm{~g} / \mathrm{kg}$ b.w. $/$ day (but higher at $37^{\circ} \mathrm{C}$ ). Although this simple calculation uses many assumptions and is subject to considerable uncertainty, it illustrates that the result of calculation 1 is likely to be an over-estimation by several orders of magnitude.

NOTE. For the vapour pressure of BPA, various numbers were reported $1.6 \times 10^{-9} \mathrm{hPa}$ at $20^{\circ} \mathrm{C}, 4.12 \times 10^{-9} \mathrm{hPa}$ at $25^{\circ} \mathrm{C}$, and $3.91 \times 10^{-7} \mathrm{~mm} \mathrm{Hg}$ at $25^{\circ} \mathrm{C}$. ANSES and EFSA indicate a value of $5.3 \times 10^{-6} \mathrm{~Pa}$ at $25^{\circ} \mathrm{C}$ (ANSES, 2013; EFSA, 2013).

Calculation 3: Calafat et al. (2009) found that total BPA (including BPA metabolites) geometric mean urinary concentration $(30.3 \mu \mathrm{g} / \mathrm{L}$ ) among prematurely born infants undergoing intensive therapeutic medical interventions was about ten times higher than that among the general population. The medical care given is not itemised and may or may not include PC incubators along with other medical devices. Fluid intake for prematurely born infants is unlikely to exceed ca. $150 \mathrm{~mL} / \mathrm{kg} \mathrm{b.w./day.} \mathrm{Insensible} \mathrm{water}$ loss in a heated incubator can be $0.6-0.7 \mathrm{~mL} / \mathrm{kg}$ b.w./hour for a larger prematurely born infant up to $2-3 \mathrm{~mL} / \mathrm{kg}$ b.w./hr for a very immature infant with immature skin and a large exposed skin area (Kliegman et al, 2011). Taking urine output as fluid input (150) minus insensible loss $(2 \times 24)$ gives ca. $100 \mathrm{~mL}$ urine/kg b.w./day. Therefore, a concentration of $30.3 \mu \mathrm{g} / \mathrm{L}$ urine would equate to approximately $3 \mu \mathrm{g} / \mathrm{kg} \mathrm{b.w./day.}$

\section{(vii) Breast pump and collection vessel made of PC}

Calculation: An analogy is made here with migration levels from PC baby bottles. EFSA (2013) considered the migration from PC baby bottle into food simulants and they derived an average migration of $0.89 \mu \mathrm{g} / \mathrm{L}$ and a high migration of $4.56 \mu \mathrm{g} / / \mathrm{L}$. Taking the average migration value along with a high level of consumption of milk by infants of 150 $\mathrm{g} / \mathrm{kg}$ b.w./day (EFSA, 2011) would give exposure of $134 \mathrm{ng} / \mathrm{kg}$ b.w./day. 


\subsubsection{BPA exposure from uses of BPA containing PVC}

Since no information on BPA release from PVC-made medical devices is available to evaluate the possible exposure to BPA originating from PVC, a parallel was drawn and information was used from the evaluation of exposure to DEHP from medical devices made of PVC, performed by the SCENIHR (SCENIHR, 2008).

Plasticised PVC is used in certain parts of medical devices for procedures such as blood transfusion, haemodialysis, parenteral nutrition or endotracheal tubing. Di(2-ethylhexyl) phthalate (DEHP) is the main plasticiser used in PVC-based medical devices. The typical concentration of DEHP in plasticized PVC is $30 \%$ (ECB, 2004).

The five DEHP-plasticised PVC food-wrap films tested by Lopez-Cervantes and PaseiroLosada (2003), contained BPA at 0,43, 96, 98 and $483 \mathrm{mg} / \mathrm{kg}$. The samples of plasticised PVC wrapping film, gloves and hose analysed by Sun et al. (2001) contained BPA at 68,61 and $290 \mathrm{mg} / \mathrm{kg}$ respectively. From these eight results, a crude average of approximately $150 \mathrm{mg}$ BPA / kg PVC was derived.

Comparing the two concentrations, with DEHP at approximately $300,000 \mathrm{mg} / \mathrm{kg}$ (i.e. $30 \%$ by weight) and BPA at ca. $150 \mathrm{mg} / \mathrm{kg}$, the concentration ratio is 2000:1. The estimation of exposure is based on the assumption that DEHP and BPA leach from plasticised PVC used in medical device, in this same ratio of 2000:1.

For adults, the SCENIHR 2008 opinion used data based on measurements of DEHP blood levels in patients before and after specific medical procedures and concluded that blood transfusions to trauma patients or during ECMO (extracorporeal membrane oxygenation) may be the short-term procedures that result in the highest acute DEHP exposure in adults, up to $10 \mathrm{mg} / \mathrm{kg} /$ day. Long-term haemodialysis was the continuously repeated procedure which may result in the highest cumulative dose of DEHP and this could be up to $2.2 \mathrm{mg} / \mathrm{kg} /$ day.

For neonates, the SCENIHR concluded that the highest short-term exposure may occur due to double volume exchange transfusion (up to $23 \mathrm{mg} / \mathrm{kg} /$ day) while ECMO is the medical treatment which may give the highest daily exposure over a prolonged period of time, at up to $14 \mathrm{mg} / \mathrm{kg} /$ day. Similarly, the SCENIHR noted that the US-FDA (2002) had estimated an upper-bound daily DEHP dose to be around $3 \mathrm{mg} / \mathrm{kg} /$ day for a newborn in the neonate intensive care unit setting, considering exposure from multiple devices. Such exposures may occur for a period of weeks or even months (SCENIHR, 2008).

The estimates of possible exposure to BPA from plasticised PVC medical devices that are calculated by a 2000 -fold down-scaling of these the SCENIHR estimates for DEHP are shown in Table 3.

Table 3: Derivation of estimates of possible exposure to BPA from medical devices made of plasticised PVC.

\begin{tabular}{|l|c|c|c|c|}
\hline & $\begin{array}{c}\text { Adults short- } \\
\text { term }\end{array}$ & $\begin{array}{c}\text { Adults longer- } \\
\text { term }\end{array}$ & $\begin{array}{c}\text { Neonates short- } \\
\text { term }\end{array}$ & $\begin{array}{c}\text { Neonates } \\
\text { longer term }\end{array}$ \\
\hline $\begin{array}{l}\text { DEHP estimate, } \\
\mu \mathrm{g} / \mathrm{kg} \text { b.w./day }{ }^{\mathrm{a}}\end{array}$ & 10,000 & 2,200 & 23,000 & 14,000 \\
\hline $\begin{array}{l}\mathrm{BPA} \quad \text { estimate, } \\
\mu \mathrm{g} / \mathrm{kg} \text { b.w./day }{ }^{\mathrm{b}}\end{array}$ & 5 & 1 & 12 & 7 \\
\hline
\end{tabular}

a) SCENIHR (2008).

b) Taking the estimates for DEHP and reducing by a factor of 2000 to account for the ratio of the concentrations of DEHP and BPA possible in plasticised PVC (300,000 and 150 $\mathrm{mg} / \mathrm{kg}$ respectively, see text) 
As stated, these estimates in Table 3 are based on the assumption that all the PVC used in the medical devices contain BPA and that the leaching propensity of BPA and DEHP from plasticised PVC are about the same. Considering the two molecules, BPA has a lower molecular weight than DEHP (228 versus 390 Daltons), but perhaps more significantly, BPA is only moderately lipophilic, whereas DEHP is strongly lipophillic (log octanol-water partition coefficients of 3.3-3.5 versus 7.5). The exposure scenarios are for leaching into blood which although largely aqueous, does have an important lipid fraction too. Therefore, in the absence of experimental data, this assumption on relative leaching rates is reasonable.

Considering the case of non-plasticised PVC, if BPA were to be present in the plastic, it is assumed that rigid PVC has only minimal uses in medical devices and also that leaching rates from the plastic would be much lower than from plasticised PVC. Thus, this potential source of exposure is not being considered further.

\subsubsection{Conclusions}

Table 4 summarises the outcome of the different exposure scenario calculations. There is considerable uncertainty with respect to the chemical composition of medical devices and their release properties with respect to BPA. For each scenario considered, there are relatively few studies reported and in most cases the studies do not have much information on how the material under study is representative of the European situation. For these reasons, the estimates of exposure have made conservative assumptions and extrapolations. Nevertheless, these figures should be viewed and used with some caution because they could underestimate or overestimate the true exposure of some patients.

Some of the estimated BPA exposures due to medical devices are in the same range as exposure via the food (EFSA, 2015). EFSA estimated the exposure to be highest for infants and toddlers among the population older than 6 months, with the highest estimated average of $375 \mathrm{ng} / \mathrm{kg}$ b.w./day and a high dietary exposure of $857 \mathrm{ng} / \mathrm{kg}$ b.w./day. The modeled dietary exposure for teenagers, adults and elderly ranged from 116 to $159 \mathrm{ng} / \mathrm{kg} \mathrm{b.w./day} \mathrm{for} \mathrm{average} \mathrm{exposure,} \mathrm{with} \mathrm{a} \mathrm{high} \mathrm{exposure} \mathrm{ranging} \mathrm{from} 335$ to $388 \mathrm{ng} / \mathrm{kg} \mathrm{b.w./day.}$

Table 4: BPA exposure from medical devices as estimated for various use scenarios.

\begin{tabular}{|l|c|c|c|c|}
\hline & \multicolumn{3}{|c|}{ BPA exposure estimation in ng/ kg b.w./ day } \\
\hline Exposure scenario & $\begin{array}{l}\text { Prematurely } \\
\text { born infant }\end{array}$ & infant & child & adult \\
\hline $\begin{array}{l}\text { External contact with a medical device } \\
\text { containing BPA } \\
\text { (short-term) }\end{array}$ & 1 & & & 0.08 \\
\hline $\begin{array}{l}\text { Contact with dental material } \\
\text { (acute exposure <24 h) }\end{array}$ & na & na & & \\
(long term) & & & 2 & 6 \\
Contact with orthodontic equipment & & & 140 & 140 \\
(acute exposure <24 h) & & & 12 & 7.5 \\
(short-term) & & & 6 \\
(long-term) & & & \\
\hline
\end{tabular}




\begin{tabular}{|l|c|c|c|c|}
\hline $\begin{array}{l}\text { Contact with an implant } \\
\text { (short-term) } \\
\text { (long-term) }\end{array}$ & & & 11 & 6 \\
\hline Hemodialysis (long-term) & & 0.8 & 0.4 \\
\hline $\begin{array}{l}\text { Prolonged surgical procedures } \\
\text { (short-term) }\end{array}$ & & 685 & 114 & 57 \\
\hline $\begin{array}{l}\text { Prolonged exposure to different sources of } \\
\text { BPA in intensive care units } \\
\text { (short-term) }\end{array}$ & 3000 & & & \\
\hline $\begin{array}{l}\text { Breast pump and collection vessel made of } \\
\text { PC } \\
\text { (short-term) }\end{array}$ & & 134 & & \\
\hline $\begin{array}{l}\text { Uses of PVC } \\
\text { (short-term) } \\
\text { (long-term) }\end{array}$ & $12000^{\mathrm{a}}$ & & & \\
\hline
\end{tabular}

a) In the absence of data on BPA, leaching properties of DEHP have been considered for the estimation.

\subsection{Toxicokinetics of bisphenol A}

\subsubsection{BPA biotransformation}

The major BPA metabolite in human is BPA-glucuronide, which is quantified in plasma and rapidly excreted in the urine; BPA-sulphate has also been detected after oral exposure as a minor urinary metabolite (Hanioka et al., 2008; Kim et al., 2003; Ye et al., 2005). In both monkeys and rats, BPA is biotransformed by the same reactions (EFSA, 2010): the predominant pathway is glucuronidation, with the sulfation reaction representing $<20 \%$ for monkeys and $<5 \%$ for rat.

The efficiency of the first pass is demonstrated by the very small amounts of unchanged parent BPA, up to $9.5 \%$ of the oral administered dose is recovered in human urine (Dekant and Völkel, 2008; Völkel et al., 2008; Ye et al., 2005), whereas a high \% of the total amount was detected as PBA-conjugates. This is especially relevant for risk assessment, since the conjugates do not retain the biological activity of the parent BPA which is the toxicologically relevant compound (Snyder et al., 2000; Shimizu et al., 2002, Willhite et al., 2008).

From in vitro data, Kurebayashi et al. (2010) calculated that $91 \%$ of the hepatic clearance is due to glucuronidation and $9 \%$ due to sulfation in human hepatocytes; a similar percentage was observed also for clearance in monkey and rat hepatocytes. In vivo a variable ratio between the two reactions was determined: indeed, glucuronides account for $80-100 \%$ and sulfates for $0-15 \%$ of the oral BPA dose measured in urine of human volunteers and in individuals unintentionally exposed to BPA (Völkel et al., 2002; Ye et al., 2005). According to a study in a Korean population, the ratio glucuronidation/sulfation is higher in men than in women (4 vs 1.5, respectively) (Kim et al., 2003). The glucuronide/sulphate ratio was not age-dependent in either species (Doerge et al., 2010b). 
Sulfation of BPA is mediated by sulfotransferases (SULT); SULT1A1, which is involved in the conjugation reaction of other phenols (Campbell et al., 1987), has been identified as the major isoform mediating BPA sulfation in the human liver, although recombinant SULT2A1 and 1E1 showed also some activity (Nishiyama et al., 2002). Among some recombinant human (UDP)-glucuronosyltransferase isoforms, UGT2B15 showed the highest activity over the range of BPA concentrations $(1-20 \mu \mathrm{M})$ tested (Hanioka et al., 2008). Other authors, by using a complementary panel of recombinant enzymes, identified a relevant role for UGT1A9 (which is expressed both in the liver and in the gastrointestinal tract) (Doerge et al., 2010b) and for UGT2B7 (Mazur et al., 2010).

It has been suggested that hepatic metabolism plays a more relevant role in humans than the intestinal one, as confirmed by recent studies carried out with human microsomes pooled from different donors and from different organs (intestine, kidney, liver, and lung), reporting that the tissue intrinsic clearances for the kidney and intestine were less than $1 \%$ of liver intrinsic clearance, whereas human lung microsomes did not show glucuronidation activity towards BPA (Mazur et al., 2010; Trdan Luŝin et al., 2012). To evaluate the possible impact of the polymorphic allelic variants genetic polymorphism in BPA conjugation, UGT2B15 variants and the $* 28$ polymorphism in the UGT1A1 gene were studied in vitro (Hanioka et al., 2011;Trdan Luŝin et al., 2012). Among the six recombinant UGT2B15 allelic variants, no significant difference in the $\mathrm{Km}$ value between wild-type and any variant UGT2B15 variant was evidenced; on the contrary UGT2B15 variants having D85Y substitution showed markedly reduced Vmax and intrinsic clearance (around $10 \%$ of the wild type enzyme) (Hanioka et al., 2011). When human liver microsomes genotyped for UGT1AS1*28 polymorphisms were tested, wild-type homozygotes and heterozygotes did not significantly differ, while polymorphic homozygotes significantly differed from both, showing $25 \%$ residual activity. The lower catalytic efficiency for glucuronidation is due to decreases in Vmax with negligible changes in $\mathrm{Km}$, consistently with the lower expression of UGT1A1 in microsomes with a *28 promoter polymorphism.

However, due to the redundancy in UGTs for conjugation, the possible compensatory activity of SULT enzymes and the overlapping substrate specificity, it is expected that a single polymorphism would not significantly affect the total BPA glucuronidation capacity of individuals (EFSA, 2010). To support this conclusion, a recent study using a human based PBPK modelling estimated a factor of 4.7 for the maximum blood concentration (Cmax) and of 4.6 for the Area Under the Curve (AUC) between human hepatic cells from 15 different donors showing low and high intrinsic clearance for BPA (Partosch et al, 2013).

In addition, starting from data on UGT variants by Hanioka et al. (2011) for an oral dose of $1 \mathrm{\mu g} / \mathrm{kg} / \mathrm{day}$, the Cmax for the UGT2B15.2 and the UGT2B15.5 variant was 3.9foldand 4.9-fold higher than that of the wild-type, respectively with AUC values 4.9 and 5.5-fold higher with respect to the wild type (Partosch et al., 2013).

The glucuronidation and the sulfate pathways are negatively correlated: by using a PBPK model it has been evidenced that in subjects with low glucuronidation capacity the fraction of dose which is metabolized to the sulfate conjugate is higher than in subjects with high glucuronidation capacity (Partosch et al., 2013).

This limited level of variability is further confirmed by the results of a recently performed biomonitoring study showing variability in BPA levels in human tissue approximately by a factor of 4, attributed to inter-individual variability in BPA metabolic disposition (Teeguarden et al., 2011).

Therefore, it can be considered that the default value of 10 used to account for kinetic interindividual variability within the general population (IPCS, 2005) can cover differences due to polymorphically expressed enzyme activity involved in BPA metabolism.

More recently, additional metabolic pathways have been reported in in vitro studies, but their relevance in vivo has not been demonstrated to date. 
Rat liver microsomes and recombinant human CYPs have been shown to biotransform BPA into hydroquinone via an ipso-substitution reaction and isopropenylphenol and hydroxycumyl alcohol (HCA) were also produced as further metabolites (due to a C-C bond scission). Formation of novel metabolites via ipso-substitution pathway was about $20 \%$ of o-OH-BPA formation, via traditional oxidative pathway of P450 (Nakamura et al., 2011). Among the panel of 12 recombinant CYP tested, only CYP3A4 and 3A5 catalysed the ipso-substitution of BPA, whereas the hydroxylation of aromatic or hydrocarbon of BPA is catalysed by CYPS of the 2C family (CYP2C18> CYP2C19> CYP2C9) (Niwa et al., 2001). Interestingly, HCA had higher ER-binding activity than BPA; for this reason, it has been claimed that this metabolic pathway plays a role in the estrogenic activity of BPA.

The formation of another active BPA metabolite, namely 4-methyl-2,4-bis(4-hydroxylphenyl) pent-1-ene (MBP), was demonstrated in incubation with rat liver S9 fraction in the presence of a NADPH-generating system (Yoshihara et al., 2001, 2004). MBP showed estrogenic activity severalfold higher than BPA in the yeast estrogen screening (YES) and in rat in vivo uterotrophic assay (Okuda et al., 2010). MBP is formed by recombination of the radical fragment of BPA, which is the one-electron oxidation product of carbon-phenyl bond cleavage, and its formation required both microsomal and cytosolic fractions (Okuda et al., 2011). Anti-CYP3A2 and anti-CYP2C11 antibodies strongly inhibited the formation of MBP, suggesting the involvement of these two isoforms in generating dimertype metabolite, whereas the exact function of cytosol is still unclear. Similar metabolic activation was also demonstrated for various other BPA-related compounds, including BPB and BPF (Okuda et al., 2011).

However, these in vitro studies have been carried out with recombinant enzymes or subcellular fractions in experimental conditions avoiding any competition with the predominant and more efficient metabolic pathways, involving direct conjugation of the parent compound. The relative importance of these reactions in actual in vivo conditions, when the other pathways are simultaneously active, is not known. Although, it is expected to be relevant under circumstances where glucuronidation is unable to work efficiently as a detoxification pathway of BPA.

\subsubsection{Toxicokinetics after oral uptake}

In humans, BPA is readily and almost completely absorbed by the oral route, as demonstrated by the high recovery in the urine of human volunteers ( $97 \%$ and $84 \%$ of the ingested dose in males and females, respectively) (Völkel et al., 2002, 2005). Since human volunteers were dosed with deuterated BPA, it was possible to differentiate between administered BPA and BPA coming from other sources. Results were consistent with studies carried out dosing nonhuman primates (Doerge et al., 2010a and b) and with some modeled data (Mielke and Gundert-Remy, 2009). Comments on these data (Vandenberg et al., 2010a, 2010b) were evaluated and the conclusion was that these comments were not scientifically justified (Hengstler et al., 2011).

In humans, both low and high single oral doses of BPA are well absorbed ( $>90 \%)$. This is a conservative estimate for neonates, since the immature pancreatic and biliary secretion and the scant presence of intestinal flora up to the $8^{\text {th }}$ month can limit the absorption of BPA in infants (Ginsberg et al., 2002). Because of high first-pass metabolism in the liver, the systemic availability of free BPA is low and varies between species. As a consequence, the half-life is short, ranging from 1 to $3.5 \mathrm{~h}$ (Völkel et al., 2002, 2008; Tsukioka et al., 2004; Shin et al., 2004), as estimated by considering the excretion data and assuming that the rate-limiting step is BPA detoxication.

Oral doses of labelled BPA (methyl-d6-BPA, $100 \mu \mathrm{g} / \mathrm{kg}$ b.w.) given to adult non human primates are nearly completely absorbed, with very low $(<1 \mathrm{nM})$ serum levels of free BPA (Doerge et al., 2010b), indicating a limited systemic availability of the parent compound and a concentration-time profile for total BPA similar to that of human volunteers 
administered a dose of 50-90 $\mu \mathrm{g} / \mathrm{kg}$ b.w. BPA (Völkel et al., 2002). The estimated halflife of $3.5 \mathrm{~h}$ for total BPA in rhesus monkey (Doerge et al., 2010b) was similar to those reported in cynomologous monkeys (4.2h; Tominaga et al., 2006), and humans (3.4h, Völkel et al., 2002). Similarly, agreement was found for other pharmacokinetic parameters obtained in non-human primates (Kurebayashi et al., 2002; Tominaga et al., 2006; Taylor et al., 2011; Tharp et al., 2012; Patterson et al., 2013). The comparison of results obtained after oral dosing and i.v. injection, expressed as the ratio $A \cup C_{\text {oral }} / A \cup C_{i} . V$. indicated also the relevance of the first-pass effect, supporting the conclusion that presystemic conjugation mainly occurring in the liver after oral administration is a crucial factor in determining the internal dose of free BPA after oral administration.

When $400 \mu \mathrm{g} / \mathrm{kg}$ bw per day of deuterated BPA was administered daily via the oral route (in fruits) to pregnant rhesus monkeys (Tharp et al. 2012) during gestational day 100 to term, the maternal serum concentrations $(\approx 4 \mathrm{~h}$ after dosing) were $0.68 \pm 0.312 \mathrm{ng} / \mathrm{ml}$ (mean \pm SEM, $n=3$ animals) for unconjugated BPA. Both methods used for BPA detection and the levels were comparable to Taylor et al. (2011).

A marked species difference exists in BPA disposition when data obtained from human and monkeys are compared to rodents, where BPA undergoes enterohepatic recirculation (Kurebayashi et al., 2003; Upmeier et al., 2000; Pottenger et al., 2000). After an oral dose, BPA is readily and completely absorbed also in rats, metabolized in the liver with a high degree of conjugation similar in the three species (>99\%): the comparison with data on free BPA in serum obtained after oral dosing and i.v. injection led to the conclusion that a high first-pass effect take place also in rats, and indeed, the total hepatic clearance for BPA conjugation as measured in vitro with cryopreserved hepatocytes is higher in rats then in monkey and human cells (Kurebayashi et al., 2010). Then, at variance with human and monkeys, BPA metabolites in rats are excreted from the liver via the bile into the gastrointestinal tract, and not in the urine. The BPA conjugates are cleaved back to BPA and the free BPA is reabsorbed (enterohepatic recirculation). As a result, BPA clearance takes longer in rats than in humans, the half-life in rodents being 19 - $78 \mathrm{~h}$ (EFSA, 2006). The occurrence of a high metabolic turnover and the occurrence of the enterohepatic recirculation was also demonstrated by a recent study using stable isotope-labeled BPA, showing a second peak in the concentration of total BPA in the plasma concentration-time profiles (Doerge et al., 2010a): this second peak was totally absent in primates kinetics.

As in rats, the plasma concentration-time profiles after an oral dose of BPA to female CD-1 mice exhibited a second peak in the concentration of total BPA, indicating the presence of the enterohepatic recirculation (Taylor et al., 2011), although the half-life ( $4 \mathrm{~h}$ ) was lower than the one exhibited by the rats. The efficiency of the pre-systemic conjugation reaction was high: only $\sim 1 \%$ of the administered dose was found as free BPA 30 minutes after dosing.

The availability of studies in mice, rats and monkeys with a common study design (same dose and vehicle, analytical methodology, model-independent pharmacokinetic analysis, age ranges), allowed reliable interspecies comparisons. Internal exposures to free BPA following oral administration are similarly low (Cmax $<10 \mathrm{pM}$ per $\mathrm{g} / \mathrm{kg} \mathrm{b.w.)}$ ) for adults of all three species, again supporting the dominant role of presystemic Phase II metabolism. Although there are major differences in BPA metabolism and disposition between rodents (enterohepatic recirculation and extensive fecal excretion of unconjugated BPA) and primates (extensive urinary excretion of conjugated BPA) that directly affects the BPA half-life, which, again, is longer in rats than in primates. Based on the analysis of oral versus intravenous toxicokinetic data (Doerge et al., 2010a, $2010 \mathrm{~b} ; 2011 ; 2012$ ), the oral systemic bioavailability of unconjugated BPA is $2.8 \%, 0.2$ $\%$ and $0.9 \%$ in rats, mice and monkeys, respectively. Gayrard et al. (2013) also reported that the absolute bioavailability for unconjugated BPA in blood was below $1 \%$ after orogastric dosing in dogs. However, after sublingual exposure systemic bioavailability was found to be much higher (70-90\% of the dose) probably due to the absence of a first pass effect (Gayrard et al., 2013). The systemic availability of 
unconjugated BPA in humans has not been evaluated experimentally, however, modelled data and controlled biomonitoring studies indicated that internal exposure in humans to unconjugated BPA is very low (1-10\%) (Mielke and Gundert-Remy, 2012; ANSES, 2013).

The comparison of BPA kinetics in adult vs. neonatal animals in the three species was also performed, highlighting another major interspecies difference related to neonatal development in the Phase II metabolism of BPA.

Oral administration of the same BPA dose $(100 \mu \mathrm{g} / \mathrm{kg} \mathrm{b.w.)}$ to PND3 (Post Natal Day 3) in rat pups produced higher Cmax in serum of total and free BPA (6- and 74-fold, respectively) when compared to adults. The fraction present as conjugates increased with age time $(93.4,96.9$ and $98.9 \%$ at PND 3, 10 and 21 , respectively), indicating a progressive development of metabolic and excretory functions toward the adult situation (when $99.5 \%$ BPA is in conjugated form). Similar results supporting the presence of first-pass metabolism, albeit at levels markedly lower than in the adult rat, were reported in neonatal rats following oral delivery of $10 \mu \mathrm{g} \mathrm{BPA} / \mathrm{kg} \mathrm{b.w}$. (Prins et al., 2011): free BPA constituted $29 \%, 21 \%$ and $31 \%$ of total BPA levels at $0.5,1$ and $2 \mathrm{~h}$, respectively. The relative deficiency in Phase II metabolism in newborn versus adults is even more pronounced in the mouse (Doerge et al., 2011b). The similarity in the toxicokinetics in newborn mice following subcutaneous (s.c.) or oral administration (AUC ratio $=1.0$ ) appeared to be mouse-specific and was explained by the metabolic immaturity, rapid oral absorption, and rapid distribution of unconjugated BPA (Doerge et al., 2011b). With advancing postnatal age, due to the increasing maturation of metabolic and elimination processes, the typical differences in toxicokinetics between the parenteral and the oral

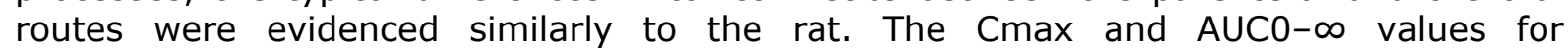
unconjugated BPA in PND 3 pups were 189- and 260-fold greater than in adults, respectively (Doerge et a.I, 2011b). However, at PND3, the internal exposure metrics (Cmax and AUC) following oral administration are similar for rats and mice, within a factor of 2 .

The pharmacokinetics of BPA in neonatal non-human primates are clearly different from neonatal rodents mainly because the degree of conjugation was not affected by developmental age and consequently there was no significant age related change in internal exposure metrics for free BPA in non-human primates.

The glucuronide/sulfate ratio was not significantly affected by age from early perinatal period to adulthood in the three species.

The comparison of age-related BPA kinetics evidenced that newborn rodents have approximately 10 times higher plasma levels of free BPA than PND5 monkeys, when treated with the same oral BPA dose (Doerge, 2010a and b; Doerge et al., 2011a). These data provide evidence for a different developmental profile of hepatic and intestinal conjugation of BPA in rodents and monkeys, consistent with literature data describing a higher degree of immaturity of rats at birth as compared to primates, in relation to UGT activity (Coughtrie et al., 1988; Matsumoto et al., 2002).

Many of the human UGT1A and 2B isoforms catalyzing BPA glucuronidation are homologous with those in monkeys and share the same tissue distribution and substrate specificity towards steroid hormones (Doerge et al., 2010b). For SULT enzymes, no agedependency has been described (Pacifici et al., 1993: Duanmu et al., 2006) and consequently, in humans the sulfation activity is comparable at birth and in the adult. These considerations are supported by the results from the study of Calafat (Calafat et al., 2009), showing that $>90 \%$ of the BPA excreted in the urine by prematurely born infants was in its conjugated (e.g. glucuronide, sulfate) form, clearly indicating that prematurely born infants are able to metabolize BPA. In addition, the concentrations of free and total BPA were linear over the range of detected BPA levels, suggesting that the enzyme(s) responsible for the conjugation of BPA were not saturated in the tested prematurely born infants. More recently, Nachman et al. (2013) measured the content of unconjugated and BPA-glucuronide in the urine of 11 healthy neonates plus 1 young infant, all but one receiving infant formula. The average concentration of BPA 
glucuronide, as measured in all of the duplicate urine samples, was $0.87 \pm 0.51 \mathrm{ng} / \mathrm{mL}$ (median: $0.66 \mathrm{ng} / \mathrm{ml}$ ). Unconjugated BPA was not found in any of the urine samples, further demonstrating that neonates and infants are capable of conjugating BPA to the BPA-glucuronide.

The large inter-species differences in internal free BPA dosimetry emphasize the importance of using physiologically based pharmacokinetic (PBPK) modelling to estimate internal exposure in adults, children and infants following different exposure scenarios. The input of enzyme kinetics parameters obtained in vitro with human samples in the PBPK models could be an important improvement in BPA risk assessments.

Although dietary BPA exposure would be a more appropriate and convenient route than bolus exposure, most of the studies have been carried out to date with oral administration of single or repeated bolus dose. Even when animals were fed a diet containing BPA (Dolinoy et al., 2007; Cox et al., 2010) serum, concentrations of BPA were not measured and it remains controversial whether the quantities of BPA supplied to mice are representative of actual exposure conditions. Only one study (Sieli et al., 2011) measured serum concentrations of isotopically tagged dimethyl-d6-BPA and its conjugates resulting from dietary exposure in female mice and compared concentrations with those in mice exposed through single oral bolus exposure, as in previous studies (Doerge et al., 2010a, 2010b; Taylor et al., 2011).

For mice receiving the oral bolus ( $20 \mathrm{mg} / \mathrm{kg}$ b.w.), maximum concentration (Cmax) of unconjugated BPA-d6 $(21.0 \pm 3.9 \mathrm{ng} / \mathrm{mL}$, mean $\pm \mathrm{SE})$ occurred within 1 hour of treatment and declined slowly thereafter, reaching barely detectable concentrations after $24 \mathrm{~h}$. The estimated dietary exposure dose was $13 \mathrm{mg} / \mathrm{kg} \mathrm{b.w}$. over the first $24 \mathrm{~h}$, and peak BPA-d6 concentration $(18.8 \pm 4.4 \mathrm{ng} / \mathrm{mL})$ was observed at $6 \mathrm{~h}$ after the initiation of the BPA-d6-supplemented diet then declining significantly by $11 \mathrm{~h}$. A similar trend was evidenced for total serum BPA, where concentrations of the conjugated form were up to 70-100 times higher than those of unconjugated BPA (Sieli et al., 2011). To compare the oral bolus and diet groups at the same external dose, the dose of the diet-exposed group was scaled to $20 \mathrm{mg} / \mathrm{kg}$ b.w., and the $A U C(0-24$ hour) was not significantly different in the dietary exposed group (227.4 \pm 41.1 and $201.0 \pm 20.6 \mathrm{ng}-\mathrm{h} / \mathrm{mL}$ in the diet and oral bolus group, respectively). A lower total BPA absorption (81\%) was estimated for the diet group: the slightly higher bioavailability associated with diet exposure $(113 \%)$ was tentatively explained by a "food-effect" (Sieli et al., 2011).

These findings and the hypothesis on the effect of food on BPA bioavailability might account for the considerable inter- and intrameal variability in BPA urinary excretion, consistent with an estimated range of exposure from 3.29 to $73.29 \mu \mathrm{g}$ found in biomonitoring studies (Teeguarden et al. 2011). An additional finding, although not explained yet, is related to concentrations of unconjugated or active BPA-d6 in the diet group which were found to be higher after 7 days of dietary exposure than at $24 \mathrm{~h}$ after exposure, an effect which has not been observed when BPA is administered as a single bolus (Doerge et al., 2010a; Taylor et al., 2011).

Different PBPK models have been developed: some are extrapolated from animal models (rat: Teeguarden et al., 2005 and Shin et al., 2010; monkey: Fisher et al., 2011) and others that are human-based models (Edginton and Ritter, 2009; Mielke and GundertRemy, 2009). Yang et al. (2013) developed a PBPK model for neonatal and adult rats with implications for the extrapolation of toxicity studies from neonatal rats to neonatal monkeys or infant humans. In view of the kinetic differences among rodents and primates, those based on primates are considered more appropriate for the extrapolation of data to the human situation, unless the rat based model is refined to account for the entrohepatic re-circulation and features typical of rodent kinetics (Shin et al., 2004) used to describe and predict the blood and tissue concentration time profiles after oral and iv doses in rats and in humans. This PBPK model was employed as an example to estimate the oral dose required to achieve the actual total BPA concentrations in human blood as reported in Korean pregnant women (Shin et al. 2010). Additionally, the Teeguarden et al. model (2005) was later extended to humans. 
The study of Churchwell et al. (2014) reports on the systemic exposures to aglycone (bioactive) and conjugated BPA in the low and high dose BPA study reported by Delclos et al. (2014). BPA was administered in a low dose range $(2.5-2700 \mu \mathrm{g} / \mathrm{kg} \mathrm{b.w./day,}$ with a total of 7 doses within this range) below the current NOAEL of $5,000 \mu \mathrm{g} / \mathrm{kg}$ b.w./day and two very high $(100,000-300,0000 \mu \mathrm{g} / \mathrm{kg}$ b.w./day) doses of BPA. Aglycone (i.e., unconjugated and active) and conjugated (i.e., inactive) BPA were evaluated by liquid chromatography electrospray tandem mass spectrometry (LCES/MS/MS). Method validation and quality control measures were applied for the BPA and other measurements. Unintentional potential BPA inputs (e.g. via diet, drinking water, vehicle, cages, bedding, and dust) were controlled and avoided by rigorous dosing solution certification and delivery. External potential sources of BPA exposure (e.g. animal bedding, polysulfone cages, silicone water bottle stoppers and drinking water) were evaluated for BPA leakage of which none was detectable above analytical blanks. Also phytoestrogen presence in feed was monitored and limited by using a soy- and alfafa-free diet. Laboratory materials used for collection and storage of samples were evaluated for possible BPA contamination. The syringes, needles, glass, and polypropylene tubes selected for use in blood collection and processing were found to contain levels of BPA at or below the daily Limit Of Blank. Some consistent material contamination was observed and these materials (e.g certain screwcap cryo-vials) were rejected. Despite these efforts to minimize the unintentional exposure of animals to BPA and the precautions taken to physically separate vehicle dosing from the BPA and EE2 doses BPA-glucuronide was detected in the serum of vehicle and naïve control animals at levels similar to those detected in animals dosed with 2.5 and $8 \mu \mathrm{g} \mathrm{BPA} / \mathrm{kg} \mathrm{b.w./day.} \mathrm{The}$ source of this of BPA exposure in these control animals was not identified. The dosing was done by gavage and started at gestational day 6 and lasted until postnatal day 90 . Blood samples were collected from study animals at various time points to evaluate systemic exposures to aglycone (bioactive) and conjugated BPA. Both the nonconjugated BPA and total BPA were measured above the levels in controls after administration of BPA in rats of approximately $80 \mu \mathrm{g} / \mathrm{kg} \mathrm{b.w./day} \mathrm{and} \mathrm{higher.} \mathrm{For} \mathrm{young}$ rats the internal exposures to the non-glucuronide BPA conjugates of BPA and EE2 (i.e. the biologically active BPA and ethinyl estradiol) was highest. Maximal serum concentrations from the two highest BPA doses and both EE2 doses were compared with concurrent levels of endogenous E2. Only the two highest doses of BPA produced a similar pattern of internal dosimetry and ER-alpha occupancy, compared with 17 $\beta$ estradiol and ethinyl estradiol, and a similar pattern of estrogenic effects in vivo as shown in the companion paper (Delclos et al., 2014). The study clearly showed that contamination with BPA can easily occur, as well as in the animals within an experiment but also in the serum samples due to collection and storage. These potential and sometimes unknown sources of BPA contamination make measurements of the nonconjugated (aglycone) BPA in blood difficult to interpret. This also may apply to studies with low dose BPA effects that did not include measurements of internal BPA exposures.

Two studies showed that contamination of plasma samples may result in a misleading value for the BPA content (Vandenberg et al., 2014, Gyllenhammar et al., 2014). The blood sampling equipment demonstrated to be a source of possible contamination (Gyllenhammar et al., 2014).

\section{Conclusion}

Major differences exist in BPA metabolism and disposition between rodents (enterohepatic recirculation and extensive fecal excretion of unconjugated BPA) and primates (extensive urinary excretion of conjugated BPA) that directly affect BPA halflife, which is longer in rats than in primates. Moreover, at the neonatal stage there is a major difference between rodents (rats and mice) and non-human primates. For neonatal rodents, the conjugation of BPA develops with increasing age being very low (especially in mice) at birth. In neonatal non-human primates (NHP), the degree of conjugation was not affected by developmental age. Even in prematurely born infants, $>90 \%$ of the BPA excreted in the urine was conjugated indicating that prematurely born infants are able to conjugate BPA. Pharmacokinetics in rats and mice with an enterohepatic recirculation 
may result in the exposure to free BPA, especially in neonatal rats and mice, being higher than in NHP and humans. Therefore, studies in postnatal rats or mice may over-predict adverse outcomes for humans (Shelnutt et al., 2013).

The large inter-species differences in internal free BPA dosimetry emphasize the importance of using physiologically based pharmacokinetic (PBPK) modelingto estimate internal exposure in adults, children and infants following different exposure scenarios. The input of enzyme kinetics parameters obtained in vitro with human samples in the PBPK models could be an important improvement in BPA risk assessments.

Based on recent new toxicokinetic data on different animal species and BPA PBPK models that have become available, EFSA has recently derived a so-called human equivalent dose (HED) for oral BPA uptake for extrapolating animal internal BPA exposure data into human internal BPA exposure data (EFSA, 2015). The HED is an accepted method for replacing the default interspecies kinetic subfactor 4 by a chemical specific factor when predicting a level without harmful effects in humans. For the HED approach, EFSA decided to use the above mentioned PBPK model of Fisher et al. (2011) to derive internal dosimetrics for oral BPA, as done by Yang et al. (2013). These models make it possible to predict the internal BPA exposures in humans for the oral route.

Churchwell et al. 2014, Vandenberg et al. 2014, Gyllenhammar et al. 2014 indicated that careful consideration is needed in BPA measurments regarding possible unintentional exposure and sample contamination during collection, storage and measurements.

\subsubsection{Toxicokinetics after uptake by other routes}

Studies on toxicokinetics of BPA available to date have demonstrated a significantly lower internal exposure to free BPA after oral intake as compared to parenteral exposure, essentially due to the highly efficient pre-systemic conjugation to glucuronides and sulfate occurring in the liver and partially in the gut after oral administration independently on the species.

Many toxicological studies showing adverse effects used s.c. injections of BPA or alternatively BPA was injected into discrete regions or delivered by osmotic pumpsto ensure reproducible dosing. There are differences due to pre-systemic clearance occurring following oral exposure and the slow release of BPA from the oil suspensions during injection/infusion, makingadirect comparison inappropriate. For route-to-route extrapolation, s.c. studies may only be useful if tissue and/or plasma values are used. However, the results of s.c. studies are appropriate for hazard identification or for risk assessment purposes for specific exposure scenarios, when BPA exposure occurs via transcutaneous or parenteral route, as it could be with some medical devices.

\subsubsection{Toxicokinetics after dermal and transcutaneous uptake}

\section{Dermal absorption}

Toxicokinetic studies in humans involving dermal exposure, indicating the extent of BPA dermal absorption and the internal dose metrics for free and conjugated BPA, are not available. However, in vivo study in rats as well as in vitro studies on cutaneous penetration using pig skin and human skin samples have been carried out.

The European Union in the RAR (ECB, 2003) estimated that the bioavailability of BPA applied on skin was around $10 \%$, but considering the physico-chemical properties of BPA, skin penetration could be expected to be higher: indeed, BPA has a moderate water solubility ( $K_{o w}$ of 3.2$), \log \mathrm{P}_{\mathrm{o} / \mathrm{w}}$ of BPA is 2.2 and a relatively low molecular weight. 
The fate of BPA after topical application or skin contact was examined in only a few studies. The oldest one was carried out using deuterated-BPA on isolated perfused bovine udders (Kietzmann et al., 1999), a model that is poorly representative of human skin andnot recommended by current guidelines for dermal absorption studies. A studywas carried out with ${ }^{14} \mathrm{C}-\mathrm{BPA}$, using full thickness human skin in vitro (Mørck et al., 2010), following OECD guideline 428 on skin penetration studies. However, in addition to poor data reporting, results were apparently obtained with an extremely high dose of BPA (17.5 mM corresponding to $3.99 \mathrm{~g} / \mathrm{L}$, inconsistent with BPA solubility of $120-300 \mathrm{mg} / \mathrm{L}$ water at $25^{\circ} \mathrm{C}$ (EFSA 2010)): the exaggerated BPA application does not allow any conclusion to be drawn from this study.

The percutaneous absorption of ${ }^{14} \mathrm{C}$-BPA was measured with porcine skin after $10 \mathrm{~h}$ of exposure (Kaddar et al., 2008). The histological and biochemical properties of porcine skin have been repeatedly shown to be close to that of human skin (Jacobi et al., 2007) as well as the thickness of both the stratum corneum and the epidermis. The proportion of radioactivity found in the receptor fluid varied from 0 to $5 \%$ of the applied dose over time (0-24 hour), with around $15 \%$ recovered in the treated skin (dermis +epidermis) at $10 \mathrm{~h}$, to give an amount of absorbed and potentially 'absorbable' material of $15-20$ $\%$. Data do not allow an absorption flux for BPA to be calculated. The apparent sequestration by the fatty compartment of the dermis could be explained with the log $\mathrm{P}_{\mathrm{o} / \mathrm{w}}>2$; on the other hand the possible biotransformation to more water-soluble metabolites through conjugation is expected to progressively increase the transfer in the receptor fluid at longer time. Unfortunately, the study did not report any measurement of possible metabolite formation and did not report on exposure times longer than $10 \mathrm{~h}$ (Kaddar et al., 2008).

BPA metabolites were measured in a recent dermal absorption study with ${ }^{14} \mathrm{C}-\mathrm{BPA}$ (50$800 \mathrm{nmol}$ ) for $72 \mathrm{~h}$, using both human skin explants and short-term cultures of pig ear skin (Zalko et al., 2011). In short-term cultures (72 h), the proportion of radioactivity diffused into culture media (trans-dermal passage in the receptor fluid) was $\approx 50-60 \%$, most of which was associated with BPA-conjugates (ca. $90 \%$ of radioactivity in the receptor fluid). However, skin viability (related to metabolic competence) did not significantly modify the absorption rate of BPAthus, questioning the results obtained on metabolites. In human skin explants, the percutaneous absorption measured at $72 \mathrm{~h}$ was $46-58 \%$ (of which ca. $10 \%$ associated to metabolites); $20-30 \%$ of the radioactivity applied on explants was retained at the application site (Zalko et al., 2011). The different results with respect to the Kaddar study could be attributed to longer exposure times (72 h).

Marquet et al. (2011) performed an in vivo and ex vivo adsorption study through rat and human skin. Rats were treated with different doses of $\left[{ }^{14} \mathrm{C}\right]$-BPA dissolved in acetone (4 $\mathrm{mg} \mathrm{BPA} / \mathrm{mL}, 50 \mu \mathrm{l} / \mathrm{cm}^{2}$ ). BPA penetrated rapidly into the skin: indeed $1 \mathrm{~h}$ post exposure the maximal penetration flux was obtained and more than $10 \%$ of the applied dose was recovered in the treated skin (with a maximum value of $19 \%$ measured after a 4 hour exposure). Moreover, a mean value of $31 \pm 10 \mu \mathrm{g} \mathrm{BPA} / \mathrm{cm}^{2}$ for the skin content did not change significantly at any time during exposure, indicating that there was no accumulation of BPA in the skin. The percentage of dermal absorption was obtained by summing up the excreted radioactivity and the one recovered in the carcass and in the skin at the administration site. Recovery was $\geq 94 \%$ in all the different experimental conditions. After an 8 hour exposure, the dermal absorption was approximately $26-29$ $\%$ (at both 8 and $72 \mathrm{~h}$ post exposure time). The amount of radioactivity in the skin decreased progressively during post exposure time (paralleled by increase in the excreted radioactivity), indicating that BPA penetrated in the skin is only partially associated to the stratum corneum (no tape stripping was applied to the treated skin, so that the stratum corneum content could not be quantitated). Dermal absorption increased linearly, with exposure time around 46 - $51 \%$ measured after 24 and 30 hour exposure time. 
The half-life of BPA after dermal absorption was estimated to be $28 \mathrm{~h}$ (compared with the half-life of $10 \mathrm{~h}$ measured after i.v. administration in the same study) (Marquet et al., 2011). When dermal absorption was measured ex vivo in human and rat skin at the studied dose, BPA was not cytotoxic for the skin and did not affect the skin's integrity.

Ex vivo and in vivo percutaneous absorption fluxes of BPA after 24 hour exposure in the rat were in the same range $\left(1.48\right.$ and $\left.2.2 \mu \mathrm{g} / \mathrm{cm}^{2} / \mathrm{h}\right)$ (Marquet et al., 2011). They found approximately 12 -fold lower flux $\left(0.12 \pm 0.09 \mu \mathrm{g} / \mathrm{cm}^{2} / \mathrm{h}\right)$ in human skin samples treated in vitro in the same conditions $\left({ }^{14} \mathrm{C}-\mathrm{BPA}\right.$ in acetone: $4 \mathrm{mg} \mathrm{BPA} / \mathrm{mL} ; 50$ and $200 \mu \mathrm{l} / \mathrm{cm}^{2}$ for human), however, inter- and intra-individual variability of up to tenfold was observed. The extent of BPA metabolism was estimated by measuring BPA metabolites in the receptor fluid after a 24 hour exposure to BPA on fresh dermatomed rat and human skin samples. For both human and rat skin, unmodified BPA accounted for more than $97 \%$ of the radioactivity detected in the receptor fluid, in contrast to results obtained by Zalko et al. (2011). However, the study's design shows weaknesses by using acetone for BPA dissolution that induces skin damage thus, promoting BPA penetration even though the viability of the skin was demonstrated.

Recently, the dermal penetration rate of ${ }^{14} \mathrm{C}$-BPA was determined in human skin in an in vitro test method performed according to the OECD Test Guideline 428 and in compliance with good laboratory practices (GLP) (Demierre et al., 2012). Results indicated a recovery in the receptor fluid of $8.6 \%$ of the applied dose $\left(1.82 \mu \mathrm{g} / \mathrm{cm}^{2}\right)$ and a total amount of bio-available BPA of $9.3 \%$ after $24 \mathrm{~h}$ incubation, thus, summing up the amount detected in the treated skin after tape stripping. However, the authors excluded the radioactivity recovered in the 15-tape stripping (34.9 \% of the applied dose), considering it entirely associated to the stratum corneum. According to the EFSA Guidance Document on dermal absorption (EFSA 2012b), only the first two tapes can be strictly considered equivalent to the stratum corneum; therefore, radioactivity recovered in tapes $3-15$ (approximately $15-20 \%$ ) should be added as potentially bioavailable. On this basis, the dermal absorption could be conservatively expected in the range of 25 - $30 \%$. EFSA recently assumed that the amount present in the stratum corneum will remain deposited on the skin surface, for reasons emerging from the PBPK modelling of dermal exposure, thus considering a dermal absorptiion of $10 \%$ (EFSA, 2015).

The differences of the Demierre et al. (2012) results with respect to previous studies have been related to the experimental model and the more realistic exposure conditions in terms of time $(24 \mathrm{~h})$ and BPA concentrations $\left(1.82 \mathrm{BPA} \mu \mathrm{g} / \mathrm{cm}^{2}\right)$. Indeed, the applied dose was slightly higher than the one estimated to be transferred from thermal paper to a single finger (1.13BPA $\mu \mathrm{g} / \mathrm{cm}^{2}$ ) and not expected to increase even in case of prolonged or repeated contacts (Biedermann et al., 2010). In addition, to be closer to sweat composition, ${ }^{14} \mathrm{C}$-BPA was dissolved in pure water and not in organic solvents (alcohol or acetone) as in the previous studies (Mørck et al., 2010; Zalko et al., 2011; Marquet et al., 2011). The application of high BPA doses can greatly influence BPA dermal penetration. Indeed, skin penetration results from passive transfer, and therefore, it is strictly dependent on the gradient between the applied concentration and the concentration in the receptor fluid. The 100-fold higher dose used in the study of Marquet et al. (2011) can thus, explain the higher flux in human skin $\left(0.12\right.$ and $0.022 \mu \mathrm{g} / \mathrm{cm}^{2} / \mathrm{h}$, respectively).

By using their previously published and validated PBPK-model (Mielke and GundertRemy, 2009), modified to include absorption through skin, Mielke et al. (2011) simulated concentrations in blood, liver and kidney after dermal intake of a daily dose of $71 \mu \mathrm{g} /$ day $(0.97 \mu \mathrm{g} / \mathrm{kg} /$ day $)$ as estimated by Biedermann et al. (2010) as the worst case exposure scenario for the dermal route with varying extent of absorption (10\%, 13\%, $46 \%$ and $60 \%)$ to account for the various data available in the literature. They compared concentrations which would result from the intake of BPA on the dermal route with those which would result from the oral administration at the TDI. Results indicated that after dermal exposur,e concentrations in blood, kidney were higher and in the liver were lower as compared to the oral route due to the high first pass in the liver. Whatever the extent 
of dermal absorption used, the dermal dose of $0.97 \mu \mathrm{g} / \mathrm{kg} /$ day gives rise to an AUC and Cmax lower (up tp 10- and even 70-fold for the highest absorption value used) than 50 $\mu \mathrm{g} / \mathrm{kg} /$ day (corresponding to the t-TDI) by the oral route.

Summarizing, the available data indicate that at the estimated exposures, BPA penetrated rapidly into the skin by passive transfer at a percentage up to $25-30 \%$ of the applied dose. The possible skin metabolism is controversial, since contrasting results were reported. However, not considering metabolism in the skin prior to systemic distribution will represent a conservative approach. Therefore, as a worst-case, a systemic bioavailability can range from $10-30 \%$ of the applied dermal dose.

\section{Subcutaneous injection}

Toxicokinetics studies were performed on rats and mice after oral and s.c. administration of $100 \mu \mathrm{g} / \mathrm{kg}$ b.w. BPA using the same experimental design (Doerge et al., 2010a, 2011a). Levels of free and conjugated deuterated BPA were measured in neonatal, immature and adult animals (post-natal day, PND 3, 10 and 21). Animals were given a single dose, which was demonstrated to be within the linear range of pharmacokinetics, so that extrapolation to lower doses is feasible.

Administration of $100 \mu \mathrm{g} / \mathrm{kg}$ b.w. BPA by s.c. injection to PND 3 rats produced 34-fold higher Cmax and 17-fold higher AUCs for free BPA compared to oral administration. The age-related changes in serum levels of free BPA observed after oral administration were not evidenced after s.c. injection. This indicates that first pass effect is not relevant after s.c. treatment and confirmed that on the contrary the first pass effect is relevant after the oral exposure even in early postnatal pups, although characterised by a lower conjugation activity.

In mice, administration of the same dose of BPA by either gavage or s.c. injection indicated that, unlike adult mice, serum levels of free BPA were consistently detected in pups of all ages at early post-dosing time points (Doerge et al., 2011a). These results are in line with previously reported data by Taylor et al. (2008), although the differences in the experimental design between the two studies make quantitative comparison quite difficult. However, this information may be relevant for the interpretation of some toxicity studies carried out by administering BPA by s.c. injection. This feature can be explained by considering that the particularly pronounced metabolic immaturity of PND 3 mice abrogates the route of administration effect observed for injection of BPA into neonatal rats (Doerge et al., 2010a) and monkeys (Doerge et al., 2010b); this difference is no longer present at PND 10 and 21 pups which have developed their conjugation activities, so that s.c. injection results as expected in higher levels of free BPA, having by-passed the pre-systemic BPA clearance in the GI tract and liver, typical of the oral route.

Similar results were obtained in another study (Prins et al., 2011), comparing BPA pharmacokinetics in neonatal rats following s.c. injection or oral delivery of $10 \mu \mathrm{g} \mathrm{BPA} / \mathrm{kg}$ b.w. Free and total BPA at max were 1.77 and $2.0 \mathrm{ng} / \mathrm{mL}$, respectively following s.c. injection with an initial peak of free BPA in blood that was not seen after oral exposure (after which max values for free and total BPA were 0.26 and $1.02 \mathrm{ng} / \mathrm{mL}$, respectively). After s.c. injection, $88 \%, 52 \%$ and $31 \%$ of BPA was in the free form at 30 min, 1 hour and $2 \mathrm{~h}$, respectively. After $2 \mathrm{~h}$, the differences in serum free BPA levels were no longer statistically different between the oral or s.c.-treated groups (Prins et al., 2011).

Pregnant adult BALB/c mice were daily injected s.c. BPA (0.1 and $1 \mathrm{mg} / \mathrm{kg}$ bw per day) on GD 1 up to 7 days after delivery (Mita et al., 2012). The dams were sacrificed 14 days after the last dose and the offspring at 3 months after delivery. The authors reported measurable unconjugated BPA levels in the tissues (liver, muscles, hindbrain and forebrain) of all animals exposed to BPA using HPLC with UV and fluorescence detection to measure BPA. Due to the analytical limitation of BPA UV detection (as discussed abobve) the results are considered of limited relevance. 


\subsubsection{Toxicokinetics after intravenous administration}

In rhesus monkeys administered with stable isotope-labeled ${ }^{13} \mathrm{C}_{12}$-BPA (100 $\mu \mathrm{g} / \mathrm{kg} \mathrm{b.w.)}$ to avoid background contamination, the total BPA levels were higher following i.v administration ( $29 \pm 19 \%$ of the administered dose at 5 min post-injection) than after oral administration of the same dose $(0.21 \pm 0.14 \%$ of total BPA at $30 \mathrm{~min}$ post-gavage), confirming that the systemic availability of free BPA in monkeys is much lower after oral exposure than after parenteral exposure (Doerge et al., 2010b).

Overall, these findings are in line with those obtained in the rat with a similar experimental design (Doerge et al., 2010a). Free BPA was rapidly eliminated from the circulation ( $>50 \%$ of circulating BPA was conjugated 5 min after i.v. injection), with a half-life of $0.66 \mathrm{~h}$ following i.v. administration; however, the fraction present as conjugated BPA was substantially lower following i.v. administration compared to oral (55 $\%$ vs. $99.5 \%$ ) due to the absence of first-pass conjugation.After i.v. exposure the percentage of free BPA was higher in adult rats than in monkeys ( $52 \pm 10 \%$ vs. $29 \pm 19 \%$ at 5 min post-injection) (Doerge et al., 2010a and 2010b).

When $\left[{ }^{14} \mathrm{C}\right]$-BPA $(10,50,100,500$, and $1,000 \mu \mathrm{g} / \mathrm{kg}$ b.w.) was administered i.v. to rats $(n=3-6)$ regardless of the dose administered, the percentage of $\left[{ }^{14} \mathrm{C}\right]$ excreted in faeces and urine were not significantly different, confirming no saturation of the excretion pathway (Marquet et al., 2011). The total recovery was in the range of $90-101 \%$ of the administered dose. Radioactivity was predominantly excreted in faeces (63-75\% of the excretion over the $72 \mathrm{~h}$ ), whereas within this period, urinary excretion accounted for about $12-22 \%$ of the total excreted $\left[{ }^{14} \mathrm{C}\right]$ and occurred essentially in the first $24 \mathrm{~h}$ after administration (Marquet et al., 2011).

The i.v. administration to female CD-1 mice of stable isotope-labeled ${ }^{13} \mathrm{C}_{12}$-BPA (100 $\mu \mathrm{g} / \mathrm{kg}$ b.w.) indicated a rapid distribution of free BPA into the tissues ( $t_{1 / 2}=0.2 \mathrm{~h}$ ) with a nearly as rapid terminal elimination phase $\left(t_{1 / 2}=0.8 \mathrm{~h}\right)$. Clearance of the parent compound was mainly due to the rapid formation of Phase II conjugates: unconjugated d6-BPA was undetectable in serum by $8 \mathrm{~h}(<0.2 \mathrm{nM})$. However, the total BPA (mostly accounting for conjugated forms) was eliminated more slowly from the circulation $\left(t_{1 / 2}=\right.$ $6.6 \mathrm{~h}$ ), due to enterohepatic recirculation, suggested by the presence of an apparent "reentry peak" at $2 \mathrm{~h}$ for total, but not for free BPA (Doerge at al, 2012). Therefore, enterohepatic re-circulation does not appear to significantly affect the serum/tissue levels of free BPA, but prolongs the elimination of BPA conjugates, as after "re-entry", a firstpass effect occurs again.

In the study of Patterson et al. (2013), $100 \mu \mathrm{g} / \mathrm{kg}$ deuterated BPA was i.v. injected in monkeys (also administered via the oral route for comparison) during late pregnancy (days 121-139 of gestation). Concentrations of unconjugated and conjugated BPA were measured at pre-dose and after approximately 5, 15, 30, 60, 120, 180, 240, 480 and 1440 minutes in the plasma of rhesus monkey dams, in the amniotic fluid and in the placenta. Concentrations were similarly measured in the plasma of the fetus (at 5, 15, $30,60,120,180,240$, and 480 minutes) by using validated LC/MS/MS methods. The kinetics in the dams treated i.v. were similar to findings in non-pregnant monkeys from a previous study (Doerge et al., 2010b). In the foetuses, plasma concentrations of unconjugated BPA were several fold lower than in the dams. The AUC measure after the oral route was 0.43 -fold of the exposure in dams treated i.v. The concentrations of both conjugated and unconjugated BPA were two orders of magnitude lower in the amniotic fluid compared to the fetal plasma. In the placenta, unconjugated BPA concentration was 2.7 fold higher than in the serum of the dams and the conjugated BPA tissue to serum ratio was 4.5. The data show that the fetus is exposed to unconjugated BPA, but to a lower extent than the dams.

When the possible sequestration by the adipose tissue was investigated following i.v. administration of deuterated BPA (100 ug/kg b.w.), limiting interference by background BPA levels, it showed that free BPA was rapidly taken up into adipose tissues $\left(t_{1 / 2}=0.07\right.$ 
hour), with maximal levels observed at 0.25 hour, followed by a slower terminal elimination phase similar to that for conjugated BPA ( $t_{1 / 2}=7.0$ hour) (Doerge at al., 2012). The maximal level of free BPA in adipose tissue did not exceed the initial measured serum level. These data support a rapid equilibrium of BPA in the blood with tissues, including fat, such that the redistribution of BPA from adipose tissue follows in parallel.

About 2.5-4 $\mathrm{ng}$ deuterated BPA/g for $100 \mathrm{mg}$ tissue were detected (Doerge et al., 2012), These values are within the same concentration range previously reported for free BPA in human adipose tissue even though different methodologies were used (Fernandez et al., 2007; Geens et al., 2012).

Despite a high adipose tissue/serum partition ratio (6.9) and minimal competence in conjugation ( $\%$ unconjugated BPA $=90 \%$ ), BPA was eliminated from adipose tissue at a rate similar to that for BPA conjugates in the whole organism. These observations confirm the non-persistent nature of BPA in vivo, mainly due to its extremely efficient conjugation and elimination in urine (primates and partially rodents) and bile (rodents only) and are in line with its aqueous solubility $(100-300 \mathrm{mg} / \mathrm{l})$, moderate lipid partitioning $(\log \mathrm{Ko} / \mathrm{w}=3.3)$, and the fat/serum distribution ratio in female rats and mice (5 and 7, respectively) (Doerge et al., 2011b; 2012). No significant bioaccumulation was reported when 11 adult female rhesus macaques were fed $400 \mu \mathrm{g} / \mathrm{kg}$ deuterated BPA (dBPA) daily for 7 days (Taylor et al., 2011).

The possible accumulation of BPA in adipose and other tissues, due to chronic exposure to low levels hypothesised by some authors (Stahlhut et al., 2009; Hugo et al., 2008; Nunez et al., 2001), is, therefore, not supported by experimental data.

\subsubsection{Toxicokinetics after inhalation}

No data are available on kinetics following inhalation exposure, which on the other hand seems not to be a relevant route of exposure for the general population (Wilson et al., 2007; Geens et al., 2009; von Goetz et al., 2010). However, this route of exposure may be relevant for medical devices such as those used for tracheal intubations. The only information available is the lack of BPA-glucuronidation shown by human lung microsomes (Trdan Luŝin et al., 2012), suggesting the absence of relevant pre-systemic inactivation after absorption through the lung epilthelium.

\subsubsection{Special considerations on susceptible populations}

Different susceptibilities for BPA have been postulated for some specific subgroups, including foetuses, infants, and older people. The age dependence of the toxicokinetics of BPA and its conjugated metabolites was studied by applying PBPK modelling to estimate levels of BPA in the blood in young children after oral exposure (Edginton and Ritter, 2009; Mielke and Gundert-Remy, 2009). The oral absorption can be considered complete, that is around $90 \%$ (although this percentage is a conservative estimate for neonates, since the immature pancreatic and biliary secretion and the scant presence of intestinal flora up to the $8^{\text {th }}$ month can limit the absorption of BPA in infants (Ginsberg et al., 2002).

Edginton and Ritter (2009) built their PBPK model using information from toxicokinetic studies in adults, and scaled to children $<2$ years of age, by replacing the age-dependent physiologic parameters relevant for kinetics in newborn. The average free BPA modelled plasma concentrations at steady state in newborns and 3 month-old infants were 11 and 2 times greater than that in adults (after a dose of $1 \mu \mathrm{g} / \mathrm{kg} \mathrm{b.w./day).}$ 
The model by Mielke and Gundert-Remy (2009) included not only the age-dependent UDPGT-mediated BPA conjugation but also took into account the sulfation pathway, assuming SULT activity toward BPA to be about $15 \%$ of that of glucuronidation, independently of the age (being already expressed at high levels starting fromintrauterine life). They reported a children/adult ratio in free BPA in blood of about 3 $(0.44 \mu \mathrm{g} / \mathrm{L}$ versus $0.13 \mu \mathrm{g} / \mathrm{L})$ : the difference between the two studies may be explained with both the pattern of exposure and the consideration of sulfation in BPA metabolism. The simulation by Mielke and Gundert-Remy underlines the importance of taking both pathways (i.e. glucuronidation and sulfation) into account and suggests that the wellexpressed sulfation activity in the newborn can compensate at least partly for the lower glucuronidation activity in neonates.

In order to perform a comparison of BPA dosimetry across species including humans, Yang et al. (2013) applied the monkey-based PBPK model of Fisher et al. (2011) for the prediction of internal dosimetrics in human newborns and adults. After a simulated daily oral administration repeated up to 14 days, the Cmax was $0.23 \mathrm{nM}$ and $0.51 \mathrm{nM}$ and the AUC was 1.53 and $1.80 \mathrm{nM}$ per hour for human newborns and adults, respectively, with a ratio lower than the ones previously predicted by Edginton and Ritter (2009) and Mielke and Gundert-Remy (2009).

Pregnant women show slightly elevated glucuronidation activity when compared to nonpregnant women, and therefore, are characterized by a higher efficiency in detoxifying BPA. This is relevant also for in utero exposure for the embryo/foetus, the exposure of which depends on maternal blood concentrations. The issue of in utero exposure has been extensively discussed in the EFSA opinion (EFSA, 2010).

There is no indication that the elderly are at risk, since their metabolic capacity associated to phase II enzymes is not affected. However, chronic diseases such as those characterised by impaired hepatic or renal functionality can be of relevance, especially if associated to the prolonged use of medical devices as for dialysis patients.

\subsubsection{Conclusions}

The available data on BPA in animals and humans indicate that there is a marked difference between the possible routes of exposures: the internal exposure after oral intake being much lower as compared to dermal or parenteral exposure. In addition, after oral administration a species-specific difference exists in BPA kinetic, indicating that BPA is eliminated faster in humans than in rats, resulting in a lower internal exposure to free BPA in humans. The direct consequence of these differences are: i) the limited representativeness of rat toxicity data in risk assessment when not associated to specific route of exposure result in in a relative higher exposure after oral intake ii) the indication that the default assumption that human are more susceptible than rodents is not completely correct at least for the kinetic component.

The oral absorption can be considered complete, that is around $90 \%$ (although this percentage is a conservative estimate for neonates, since the immature pancreatic and biliary secretion and the scant presence of intestinal flora up to the $8^{\text {th }}$ month can limit the absorption of BPA in infants (Ginsberg et al., 2002). However, the systemic bioavailability of free BPA is dramatically reduced by the first pass effect to $2.8 \%, 0.2 \%$, $0.9 \%$ and less than $1 \%$ in rats, mice, monkeys, and dog, respectively. The systemic availability of unconjugated BPA in humans has not been evaluated experimentally, however, modelled data indicated that internal exposure in humans to unconjugated BPA is very low $(1-10 \%)$. This estimate was confirmed by results from controlled biomonitoring studies in humans showing that unconjugated BPA in serum is below the LOD of $0.3 \mathrm{ng} / \mathrm{ml}(=1.3 \mathrm{nM})$, confirming that internal exposure to unconjugated BPA is extremely low. 
After dermal exposure, the absorption fraction can conservatively be considered in the range of $10-30 \%$ of the applied dose, which is directly systemically bioavailable.

For all the parenteral routes of exposure (including i.v., i.p., trandermal or subcutaneous), the chemical is $100 \%$ systemically bioavailable: however, the clearance of free BPA from the circulation appeared to be quite fast, as indicated by controlled studies in non human primates showing a half-life of $0.66 \mathrm{~h}$ with $>50 \%$ of circulating BPA conjugated 5 min after i.v. injection.

The available modelled data, obtained considering after oral exposure, also point out that newborns and babies up to 6 months constitute a potentially susceptible subpopulation due to immature BPA metabolism. However, the default uncertainty factor that is used to account for the toxicokinetic variability in the general population seems to be large enough to cover the variability in the newborn population exposed via the oral route. Analogously, inter-individual differences in the expression of the isoenzyme mainly responsible for BPA glucuronidation are within a factor of 4 , again covered by the usual uncertainty default factor, at the estimated dietary exposures.

Based on animal data and PBPK modelling, it is possible to provide internal dose metrics for neonatal-to-adult stages and for different routes of exposure. More recently EFSA derived a human equivalent dose (HED), a concept that can be used to derive internal human exposure data for BPA, to be applied to Points of Departure derived from animal studies (EFSA 2014, 2015).

Careful consideration is needed in BPA measurments regarding possible unintentional exposure in animal experiments and serum sample contamination during collection and storage.

\subsection{Toxicity}

\subsubsection{General toxicity studies}

\subsubsection{Acute toxicity}

Oral LD50 values above $2,000 \mathrm{mg} / \mathrm{kg} \mathrm{b.w./day} \mathrm{were} \mathrm{reported} \mathrm{in} \mathrm{the} \mathrm{rat} \mathrm{and} \mathrm{mouse,} \mathrm{and}$ dermal LD50 values above $2,000 \mathrm{mg} / \mathrm{kg}$ b.w./day have been reported in the rabbit. For inhalation, a $6 \mathrm{~h}$ exposure to $170 \mathrm{mg} / \mathrm{m}^{3}$ (the highest attainable concentration) produced no deaths in rats; slight and transient slight nasal tract epithelial damage was observed. These data indicate that BPA is of low acute toxicity by all routes of exposure relevant to human health (EC, 2003, 2008, 2010a,b).

The effects of single oral exposure to BPA in humans are not well documented. In a kinetic study in healthy volunteers, a dose of $5 \mathrm{mg} \mathrm{BPA}$ (range 54.3 to $87.7 \mu \mathrm{g} / \mathrm{kg}$ ) was well tolerated (Völkel et al., 2002).

\subsubsection{Chronic toxicity (repeated-dose) studies}

Mice and rats

Oral

Several repeated dose toxicity studies have been performed in rodents which wereextensively revised in the previous evaluations and are, therefore, not all recapitulated here.

Dietary studies in mice indicated that the liver is a target organ in this species, with changes being observed in the size and nucleation state of hepatocytes in 2-year and 90- 
day studies (US NTP, 1982). It was not possible to identify a no effect level for males in the 90 day study as the effect was observed at all dose levels used in males, the lowest doses being $120 \mathrm{mg} / \mathrm{kg} \mathrm{b.w./day.} \mathrm{In} \mathrm{females,} \mathrm{in} \mathrm{the} \mathrm{2-year} \mathrm{study} \mathrm{a} \mathrm{no-adverse-effect}$ level of $650 \mathrm{mg} / \mathrm{kg} \mathrm{b.w./day} \mathrm{was} \mathrm{established} \mathrm{based} \mathrm{on} \mathrm{reduction} \mathrm{of} \mathrm{body} \mathrm{weight} \mathrm{gain.}$ Thus, the LOAEL in males is $120 \mathrm{mg} / \mathrm{kg} \mathrm{b.w./day} \mathrm{and} \mathrm{the} \mathrm{NOAEL} 650 \mathrm{mg} / \mathrm{kg} \mathrm{b.w./day} \mathrm{in}$ females. A NOAEL of $74 \mathrm{mg} / \mathrm{kg}$ b.w./day has been established for rats from a 2-year study based on reduced bodyweight gain at the next dose level of $148 \mathrm{mg} / \mathrm{kg} \mathrm{b} . \mathrm{w} . / \mathrm{day}$.

Tyl et al. (2002, 2008) conducted a dose-range finding study and two large multigenerational studies in rats and mice using dietary administration of BPA with doses ranging from 1 or $3 \mu \mathrm{g} / \mathrm{kg}$ b.w./day up to 500 or $600 \mathrm{mg} / \mathrm{kg}$ b.w./day. These studies demonstrated effects on the liver, kidney and body weight at doses of $50 \mathrm{mg} / \mathrm{kg}$ b.w./day and higher. Chronic inflammation of the liver was seen from $50 \mathrm{mg} / \mathrm{kg} \mathrm{b} . \mathrm{w} . / \mathrm{day}$ in the 3-generation study, but with no convincing dose-response relationship. These liver effects in rats were thus considered to be background variation and not treatmentrelated. Renal tubule degeneration and other effects on the kidney was also seen in this 3 -generation study in females at $500 \mathrm{mg} / \mathrm{kg} \mathrm{b.w./day} \mathrm{but} \mathrm{not} \mathrm{at} 50 \mathrm{mg} / \mathrm{kg} \mathrm{b.w./day.}$ Hence, the NOAEL for kidney effects is $50 \mathrm{mg} / \mathrm{kg} \mathrm{b.w./day.} \mathrm{In} \mathrm{mice,} \mathrm{the} \mathrm{NOAEL} \mathrm{based} \mathrm{on}$ effects on liver was $5 \mathrm{mg} / \mathrm{kg}$ b.w./day. Stump et al. (2010), used a wide dose range in rats, performing a study on neurotoxicity according to OECD 426 and based on reduced body weight or body weight gain respectively identified a lowest no-observed-adverseeffect level (NOAEL) of $5.85 \mathrm{mg} / \mathrm{kg}$ b.w./day.

The Tyl et al., 2008 study was used by EFSA for calculation of the BMDL ${ }_{10}$ that was used for the estimation of the human equivalent dose (HED) and the risk assessment of BPA in their recent evaluation (EFSA 2015).

Inhalation

In an inhalation study in rats, slight inflammation and hyperplasia of the olfactory epithelium were observedat an exposure level of $50 \mathrm{mg} / \mathrm{m}^{3}(6 \mathrm{~h} /$ day, 5 days/week for 13 weeks) the NOAEL being $10 \mathrm{mg} / \mathrm{m}^{3}$ (EC, 2003, 2008).

\section{Dogs}

In a 90-day dietary study in dogs, a no effect level of approximately $80 \mathrm{mg} / \mathrm{kg} \mathrm{b.w./day}$ was identified, with increases in relative liver weight being the only finding observed at approximately $270 \mathrm{mg} / \mathrm{kg}$ b.w./day. In the absence of histopathology, this finding is of uncertain toxicological significance. (EC 2003, 2008).

In conclusion, BPA is of low acute toxicity, and the lowest NOAEL for subchronic exposure currently available is approximately $5 \mathrm{mg} / \mathrm{kg} \mathrm{b.w./day,} \mathrm{based} \mathrm{on} \mathrm{effects} \mathrm{on} \mathrm{the} \mathrm{liver} \mathrm{as}$ target organ, as identified in several studies. The next lowest NOAEL is $50 \mathrm{mg} / \mathrm{kg}$ b.w./day, based on effects on the kidney.

\subsubsection{Genotoxicity}

\section{$\underline{\text { In vitroassays }}$}

Studies of the potential of BPA to induce mutations, chromosomal aberrations, sister chromatid exchange and transformation in a variety of in vitro test systems are largely negative, including studies with Salmonella typhimurium, Chinese hamster V79 cells, Syrian hamster embryo cells and mouse lymphoma cells (NTP, 2008). However, deoxyribonucleic acid (DNA) damage was induced by BPA in MCF-7 and MDA-MB-231 cells (Iso et al., 2006). DNA adduct formation in Syrian hamster ovary cells (Tsutsui et al., 1998, 2000) and a number of positive findings have been reported for the potential for BPA to inhibit purified microtubule polymerization, affect the spindle apparatus and produce aneuploidy in in vitrostudies with Chinese hamster V79 cells or oocytes from BALB/C or MF1 mice (US NTP, 2008). 
BPA appears to have demonstrated aneugenic potential in vitro, positive results being observed without metabolic activation in a micronucleus test in Chinese hamster V79 cells and in a non-conventional aneuploidy assay in cultured Syrian hamster embryo cells. Additionally, in cell-free and cellular systems there is information that shows BPA disrupts microtubule formation. BPA as been shown to produce adduct spots in a postlabelling assay with isolated DNA and a peroxidase activation system, but it does not appear to produce either gene mutations or structural chromosome aberrations in bacteria, fungi or mammalian cells in vitro. (EFSA 2010)

In the study by Tiwari et al. (2012), negative results were obtained at concentrations up to $200 \mu \mathrm{g} /$ plate in an Ames assay using tester strains of S. typhimurium TA 98, TA 100 and TA 102 in the presence and absence of S9 metabolic activation.

In the study by Audebert et al. (2011), BPA was shown to be negative for induction of phosphorylated histone $\gamma-\mathrm{H} 2 \mathrm{AX}$, a marker for induction of DNA double strand breaks, in HepG2and LS174T (human epithelial colorectal adenocarcinoma cells).

Concerning the studies available before 2010, EFSA (2010) noted that the conduct of these studies had some deficiencies and the negative results cannot be taken as conclusive. BPA does not appear to be aneugenic in vivo, because a recently conducted, standard mouse bone marrow micronucleus test has given a negative result (EFSA 2010). Any aneugenic potential of BPA seems to be limited to in vitro test systems and is not of concern when follow up in vivo studies show negative results. The relevance of the finding that BPA can produce rat hepatic DNA adduct spots in a postlabelling assay is not entirely clear. However, given the absence of positive results for gene mutation and clastogenicity in cultured mammalian cell tests, it seems unlikely that these are of concern for human health. The newer studies are not indicative of an in vitro genotoxicity of BPA.

\section{$\underline{\text { In vivo studies }}$}

Hunt et al. (2003) investigated the effects of short-term, low-dose exposure to BPA on the meiotic processes of female mice during the final stages of oocyte growth. Although BPA can affect chromosomal structure during replication in in vitro studies, the outcomes of similar assessments when the chemical is administered to laboratory mice are inconsistent and inconclusive. The striking findings of meiotic aneuploidy in oocytes of mice (Hunt et al., 2003; Susiarjo et al., 2007) have not been independently replicated, and the failure to observe clear effects on fertility or cancer associated with BPA exposures during development suggests that the findings are of limited biological significance.

In the study by Masuda et al. (2005) intended to simulate stomach environment and its influence on genotoxicity by studying the reaction of BPA and nitrite under acidic conditions, BPA did not induce micronuclei in peripheral blood reticulocytes when administered at $228 \mathrm{mg} / \mathrm{kg} \mathrm{b.w}$. by oral gavage to male ICR mice.

Following several treatment modalities (single oral gavage treatment at 0.2 and 20 $\mathrm{mg} / \mathrm{kg}$ b.w., seven daily administrations of $0.04 \mathrm{mg} / \mathrm{kg}$ b.w. by oral gavage or seven weeks drinking water at $0.5 \mathrm{mg} / \mathrm{l}$ ) Pacchierotti et al. (2008), evaluated potential aneugenic effects of BPA on mouse female germ cells. Following six daily administrations of BPA of $0.002,0.02$ and $0.2 \mathrm{mg} / \mathrm{kg}$ b.w. by oral gavage, effects on male germ and induction of micronuclei in bone-marrow cells were investigated (Pacchierotti et al., 2008). No significant induction of hyperploidy or polyploidy in oocytes and zygotes was observed at any dose-level and treatment condition employed. Similarly, no induction of hyperploidy or polyploidy in epydidimal sperms was observed in male mice. Negative results on induction of micronuclei in bone marrow cells of male mice were also obtained.

Izzotti et al. (2009) studied BPA induction of DNA adducts, detected by ${ }^{32} \mathrm{P}$-postlabelling in liver and mammary cells of female CD-1 mice (BPA in drinking water, dose equivalent to $200 \mathrm{mg} / \mathrm{kg}$ b.w./day for 8 days). Treatment related bulky DNA adducts (two major DNA adducts) were observed in liver and in mammary cells. The authors attributed the 
formation of adducts to the reactive metabolite BPA-3,4-quinone (BPAQ). However, as the chemical characterization of DNA adducts was not performed, unspecific covalent binding to DNA cannot be excluded.

The results of Naik et al. (2009) indicate that no significant increases of chromosomal aberrations or micronuclei were induced at 10,50 and $100 \mathrm{mg} / \mathrm{kg} \mathrm{b.w.} \mathrm{or} \mathrm{five} \mathrm{daily}$ administrations at $10 \mathrm{mg} / \mathrm{kg} \mathrm{b.w}$. by oral gavage. It can be concluded that BPA under the reported experimental conditions was not clastogenic and did not elicit micronuclei induction, which would be indicative of a clastogenic and/or aneugenic potential at doselevels employed.

De Flora et al. (2011) did not find induction of micronuclei in bone marrow cells and positive comet assay in peripheral blood cells after a treatment with $200 \mathrm{mg} / \mathrm{kg} \mathrm{b} . \mathrm{w}$. BPA for 10 consecutive days.

Ulutaş et al. (2011) studied the effect of BPA (oral administration of 125 and $250 \mathrm{mg} / \mathrm{kg}$ b.w./day for four weeks) in the alkaline comet assay. No effect was observed at the lower dose-level (125 mg/kg b.w./day), whereas at $250 \mathrm{mg} / \mathrm{kg}$ b.w., the positive results might be explained by cytotoxicity which was not clearly ruled out.

Dobrzyńska and Radzikowska (2013) showed that BPA induced statistically significant increases of DNA breaks (DNA tail moment in the alkaline comet assay) in male germ cells at $24 \mathrm{~h}$ and 5 weeks from last administration of test compound and in bone marrow, spleen, kidney and lung cells at $24 \mathrm{~h}$ from last administration of $5,10,20$ or $40 \mathrm{mg} / \mathrm{kg}$ b.w. per day in drinking water for 2 weeks. The increases observed were not doserelated and were obtained following collection of organs/tissues $24 \mathrm{~h}$ or 5 weeks after last administration. Significant increases observed indiscriminately at $24 \mathrm{~h}$ and at 5 weeks from last administration raise questions about the reliability of the results. In addition, the authors did not evaluate cytotoxicity. Hence, no conclusion could be drawn from this study.

Tiwari et al. (2012) investigated oral exposure of BPA investigated for induction of micronuclei and structural chromosome aberrations in bone marrow cells and primary DNA damage in blood lymphocytes using single cell gel electrophoresis ("Comet assay"). Furthermore, plasma concentrations of 8-hydroxydeoxyguanosine (8-OHdG), lipid peroxidation and glutathione activity were also evaluated to assess potential induction of oxidative DNA damage in rats following the oral administration of test compound once a day for 6 consecutive days at dose-levels of $2.4 \mu \mathrm{g}, 10 \mu \mathrm{g}, 5 \mathrm{mg}$ and $50 \mathrm{mg} / \mathrm{kg} \mathrm{b.w./day}$. The observed increases achieved statistical significance at a dose-level of $10 \mu \mathrm{g} / \mathrm{kg}$ b.w./day and higher. Similarly, the analysis of primary DNA damage evaluated by comet assay, in isolated peripheral blood lymphocytes showed marked and dose-related increases.However, the study of Tiwari et al. (2012) hasseveral shortcomings which include the staining procedure used to detect micronuclei in the bone marrow cells, the micronuclei data reporting, the observation of chromosomal aberration figures which are not generally induced by known chemical clastogens, and the absence of evaluation of cytotoxicity in the comet assay. Hence, the reported dose-related increases of both micronuclei and structural chromosome aberrations in bone marrow cells in a dose range between $10 \mu \mathrm{g} / \mathrm{kg} \mathrm{b.w}$. up to $50 \mathrm{mg} / \mathrm{kg} \mathrm{b.w.} \mathrm{for} \mathrm{six} \mathrm{days} \mathrm{are} \mathrm{difficult} \mathrm{to} \mathrm{interpret} \mathrm{and}$ cannot be considered reliable in view of the methodology used. In a preliminary study with two doses (10 $\mu \mathrm{g} / \mathrm{kg}$ b.w./day and $5 \mathrm{mg} / \mathrm{kg} \mathrm{b.w./day)} \mathrm{in} \mathrm{male} \mathrm{rats,} \mathrm{oral} \mathrm{BPA} \mathrm{by}$ gavage for 6 days induced for both doses a decrease in sperm production (Tiwari and Vanage 2013). In addition, the highest dose of $5 \mathrm{mg} / \mathrm{kg}$ b.w./day induced sperm DNA damage as demonstrated in a comet assay.

\section{Overall Conclusions on genotoxicity of BPA}

The genotoxicity of BPA has been reviewed elsewhere (Haighton et al., 2002; ECB, 2003; EFSA, 2006; US NTP-CERHR, 2008; EFSA, 2010; WHO, 2010). Additional and new publications were reported and evaluated in this part. BPA did not induce in vitro gene mutation in bacteria (Masuda et al., 2005; Tiwari et al., 2012) and in vivo micronuclei in 
rodent bone marrow assays (Masuda et al., 2005; Pacchierotti et al., 2008; Naik et al., 2009; De Flora et al., 2011). BPA is aneugenic in an in vitro study in mammalian cells by Johnson and Parry (2008) due to a spindle disrupting effects of BPA. This effect has also been demonstrated by induction of colchicine-like metaphases (C-metaphases) in mammalian cells in vitro (Tayama et al., 2008) and in vivo by induction of prematurely separated chromatids in metaphase II of mouse oocytes (Pacchierotti et al., 2008) and cmetaphases in mouse bone marrow cells in vivo (Naik et al., 2009). Obviously, BPA does not interact with DNA directly but it acts on the mitotic spindle apparatus, an effect which is thought to be thresholded (COM Guidance on a Strategy for Testing of Chemicals for Mutagenicity, Department of Health, UK, 2000).

The large margin between the dose-levels found negative in vivo for induction of aneuploidy in rodent germ cells (Pacchierotti et al., 2008) and for induction of micronuclei in somatic bone marrow cells (Masuda et al., 2005; Pacchierotti et al., 2008; Naik et al., 2009; De Flora et al., 2011) provides adequate reassurance that aneugenic effects of BPA in vivo is not substantiated. Overall, the results show that a genotoxic effect is unlikely.

In conclusion, BPA is not likely to pose a genotoxic hazard to humans.

\subsubsection{Carcinogenicity}

\section{BPA studies}

In the traditional rodent cancer bioassay (US NTP, 1982), BPA was tested in F 344 rats at 2 dose levels of approximately 74 and $140 \mathrm{mg} / \mathrm{kg} \mathrm{b.w./day,} \mathrm{and} \mathrm{in} \mathrm{B6C3F1} \mathrm{mice} \mathrm{at} \mathrm{dose}$ levels of 130 and $835 \mathrm{mg} / \mathrm{kg}$ b.w./day in male mice and 835 and $1670 \mathrm{mg} / \mathrm{kg} \mathrm{b.w./day} \mathrm{in}$ female mice. The number of animals was 50 per group. Gross morphological and histopathological investigations were performed, whereas no clinical chemistry or haematological investigations were performed, which was the standard procedure at that time. There was a marginally increased rate of leukemias in male rats, which disappeared after Bonferroni adjustment; leukemias were also seen in female rats and male mice; the increased incidence not being statistically significant. Statistically significant increase interstitial cell carcinomas of the testes were attributed to the ageing of the rats. It should be mentioned that the part of the study done in rats is somewhat compromised by the fact that among male rats in the control group, only about $50 \%$ of the rats survived, whereas in femalesthe survival rate was about $70 \%$. In mice, survival was about $80 \%$.

The authors of the study concluded that under the condition of the bioassay (US NTP, 1982 ) there was no convincing evidence that the substance was carcinogenic to $F 344$ rats or B6C3F1 mice of both sexes. Other assessments (e.g. FAO/WHO, 2011) followed this interpretation of the study results.

No inhalation or dermal carcinogenicity studies are available, although in repeat exposure inhalation toxicity studies, BPA did not exhibit properties that raise concern for potential carcinogenicity. Only minimal inflammation was seen in the upper respiratory tract at 50 $\mathrm{mg} / \mathrm{m}^{3}$ in a 13 -week study and the severity did not increase up to concentrations close to the maximum attainable concentration in the experimental system used, $150 \mathrm{mg} / \mathrm{m}^{3}$ (EC 2003).

The United States National Toxicology Program (NTP) bioassay did not include exposures during the peri-natal period. Later studies addressed this point.

Studies that included perinatal (gestational and/or lactational) exposures to BPA (oral doses to the dam from $\sim 10$ to $250 \mu \mathrm{g} / \mathrm{kg}$ b.w./day) have reported, among other lesions, proliferation of mammary ductal epithelium and squamous metaplasia of prostatic epithelium in offspring, conditions suggested to predispose to neoplasia (Timms et al., 2005; Moral et al., 2008). Additional treatments with initiating or promoting agents have 
led to earlier onset of mammary tumours (Jenkins et al., 2009) or prostatic intraepithelial neoplasia (Prins et al., 2011). Further studies used transgenic animals. An overview of carcinogenic studies including those using a co-treatment with a known carcinogen are presented in table 5. 
Table 5: Pre- and perinatal exposure in carcinogenic studies with and without inducing agents

\begin{tabular}{|c|c|c|c|c|c|}
\hline Author & Species & $\begin{array}{l}\text { Dose ( } \mathrm{mg} / \mathbf{~ k g} \\
\text { b.w./ day) }\end{array}$ & $\begin{array}{l}\text { Treatment } \\
\text { duration }\end{array}$ & Co-treatment & Outcome \\
\hline \multicolumn{6}{|l|}{ All organs } \\
\hline $\begin{array}{l}\text { Takashima et } \\
\text { al., } 2001\end{array}$ & rat & $\begin{array}{l}0,400-600 \text { (one } \\
\text { dose level) in } \\
\text { drinking water }\end{array}$ & $\begin{array}{l}10 \text { week } \\
\text { before mating } \\
\text { until end of } \\
\text { lactation }\end{array}$ & $\begin{array}{l}\text { N-nitrosobis-(2- } \\
\text { hydroxypropyl)- } \\
\text { amine }\end{array}$ & $\begin{array}{l}\text { BPA during development does not exert promoting effects on } \\
\text { BHP-induced thyroid, lung, liver, thymus and esophagus } \\
\text { carcinogenesis }\end{array}$ \\
\hline $\begin{array}{l}\text { Ichihara et al., } \\
2003\end{array}$ & rat & $\begin{array}{l}0,0.05,7.5,30, \\
\text { and } 120 \text { by } \\
\text { gavage }\end{array}$ & $\begin{array}{l}\text { pregnancy } \\
\text { and lactation }\end{array}$ & $\begin{array}{l}\text { 3,2-dimethyl-4- } \\
\text { aminobiphenyl }\end{array}$ & $\begin{array}{l}\text { Without DMAB treatment, incidences of prostatic intraepithelial } \\
\text { neoplasia (PIN), carcinoma, and atypical hyperplasia were not } \\
\text { increased }\end{array}$ \\
\hline \multicolumn{6}{|l|}{ Uterus } \\
\hline $\begin{array}{l}\text { Yoshida et al., } \\
2004\end{array}$ & rat & $\begin{array}{l}0,0.006 \text { and } 6 \\
\text { oral }\end{array}$ & $\begin{array}{l}\text { GD } 2 \text { to PND } \\
21\end{array}$ & $\begin{array}{l}\text { N-ethyl-N'-nitro- } \\
\text { nitrosoguanidine }\end{array}$ & $\begin{array}{l}\text { incidence of uterine preneoplastic or neoplastic lesions induced } \\
\text { by ENNG was not increased by BPA exposure }\end{array}$ \\
\hline \multicolumn{6}{|c|}{ Leydig cell division } \\
\hline $\begin{array}{l}\text { Nanjappa et al., } \\
2012\end{array}$ & rat & $\begin{array}{l}0.0025,0.025 \\
\text { gavage }\end{array}$ & $\begin{array}{l}\text { GD } 12 \text { to PND } \\
21\end{array}$ & & $\begin{array}{l}\text { prepubertal period and increased Leydig cell numbers were } \\
\text { shown in the testes of adult male rats at } 90 \text { days }\end{array}$ \\
\hline \multicolumn{6}{|l|}{ Prostate } \\
\hline Ho et al., 2006 & rat & $\begin{array}{l}0.01 \text { sub- } \\
\text { cutaneous }\end{array}$ & $\begin{array}{l}\text { PND 1,3 and } \\
5\end{array}$ & $\begin{array}{l}17 \beta \text {-estradiol }(E) \\
\text { and testosterone } \\
(\mathrm{T}) \text { by sub- } \\
\text { cutaneous } \\
\text { implantation for } \\
16 \text { weeks }\end{array}$ & $\begin{array}{l}\mathrm{BPA} / \mathrm{E}+\mathrm{T} 10 / 10 \\
\mathrm{E}+\mathrm{T} 4 / 10 \\
\text { prostatic intraepithelial neoplasia (PIN) }\end{array}$ \\
\hline \multirow[t]{2}{*}{$\begin{array}{l}\text { Prins et al., } \\
2011\end{array}$} & rat & $\begin{array}{l}0.01 \text { sub- } \\
\text { cutaneous } \\
0.01 \text { oral }\end{array}$ & $\begin{array}{l}\text { PND 1,3 and } \\
5\end{array}$ & $\begin{array}{l}17 \beta \text {-estradiol }(E) \\
\text { and testosterone } \\
(\mathrm{T}) \text { by sub- } \\
\text { cutaneous } \\
\text { implantation for } \\
16 \text { weeks }\end{array}$ & $\begin{array}{l}\text { PIN } \\
\text { BPA/E+T >E+T; } \\
\text { no difference between oral and sc }\end{array}$ \\
\hline & rat & $\begin{array}{l}0 \text { or } 0.025 \\
\text { subcutaneous }\end{array}$ & GD 8-23 & $\begin{array}{l}\mathrm{N} \text {-nitroso- N- } \\
\text { methylurea }\end{array}$ & $\begin{array}{l}\text { BrdU/apoptosis ratio was significantly increased and apoptosis } \\
\text { was significantly decreased in mammary parenchyma and } \\
\text { stroma }\end{array}$ \\
\hline
\end{tabular}


The safety of the use of bisphenol $A$ in medical devices

\begin{tabular}{|c|c|c|c|c|c|}
\hline $\begin{array}{l}\text { Tang et al., } \\
2012\end{array}$ & rat & $\begin{array}{l}0.01 \text { sub- } \\
\text { cutaneous } \\
\text { admin-istration } \\
\text { in three doses }\end{array}$ & $\begin{array}{l}\text { PND 1, 3, and } \\
5\end{array}$ & $\mathrm{E}+\mathrm{T}$ & $\begin{array}{l}\text { in prostate hypomethylation of the promoter of nucleosom } \\
\text { binding protein-1 persisting until day } 100 \text {; hypermethylation of } \\
\text { hippocalcin-like } 1 \text { which shows changes throughout life; } \\
\text { persistent overexpression of four of eight genes functioning in } \\
\text { methylation/demethylaton of DNA }\end{array}$ \\
\hline \multicolumn{6}{|c|}{ Mammary gland } \\
\hline $\begin{array}{l}\text { Durando et al., } \\
2007\end{array}$ & rat & $\begin{array}{l}25 \mu \mathrm{g} / \mathrm{kg} \text { b.w. } \\
\text { subcutaneous }\end{array}$ & GD8 to GD23 & $\begin{array}{l}\text { additional } \\
\text { groups: NMU ( } 25 \\
\mathrm{mg} / \mathrm{kg})\end{array}$ & $\begin{array}{l}\text { 1. No NMU tretamen: } \\
\text { increased proliferation/apoptosis ratio } \\
\text { 2-NMU treatment: } \\
\text { increased percentage of hyperplastic ducts and induced the } \\
\text { development of neoplastic lesions }\end{array}$ \\
\hline $\begin{array}{l}\text { Murray et al., } \\
2007\end{array}$ & rat & $\begin{array}{l}0,0.0025, \\
0.025,0.250, \text { or } \\
1 \text { subcutaneous }\end{array}$ & $\begin{array}{l}\text { GD } 9 \text { through } \\
\text { PND } 1\end{array}$ & & $\begin{array}{l}\text { 3-4-fold increase mammary hyperplastic ducts in all dose } \\
\text { groups; } \\
\text { increased cribriform structures in the } 0.250 \text { and } 1 \mathrm{mg} / \mathrm{kg} \text { b.w. }\end{array}$ \\
\hline $\begin{array}{l}\text { Vandenberg et } \\
\text { al., } 2008\end{array}$ & mice & $\begin{array}{l}0.25, \quad 2.5, \\
\mu g / k g \\
\text { b.w SC }\end{array}$ & $\begin{array}{l}\text { GD } 8 \text { through } \\
\text { PND } 16\end{array}$ & & intraductal hyperplasias, alveolar buds \\
\hline $\begin{array}{l}\text { Moral et al., } \\
2008\end{array}$ & rat & $\begin{array}{l}25, \quad 250 \mu \mathrm{g} / \mathrm{kg} \\
\text { b.w. orally }\end{array}$ & $\begin{array}{l}\text { GD } 10 \text { to } \\
\text { delivery }\end{array}$ & & increase in mammary hyperplastic ducts \\
\hline $\begin{array}{l}\text { Jenkins et al., } \\
2009\end{array}$ & rat & $\begin{array}{l}0,25, \text { and } 250 \\
\mu \mathrm{gPA} / \mathrm{kg} \text { b.w. } \\
\text { orally dosing to } \\
\text { dams }\end{array}$ & $\begin{array}{l}\text { during } \\
\text { lactation } \\
\text { (PND1- } \\
\text { PND20) }\end{array}$ & $\begin{array}{l}\text { additional groups } \\
\text { received } 30 \mathrm{mg} \\
\text { DMBA/kg body } \\
\text { weight On PND } \\
50\end{array}$ & $\begin{array}{l}\text { 1. no DMBA treatment: } \\
\text { increased cell proliferation and decreased apoptosis at } 50 \text { but } \\
\text { not } 21 \text { days postpartum } \\
\text { 2. DMBA treatment: } \\
\text { dose-dependent increase in mammary tumour multiplicity and } \\
\text { reduced tumour latency compared to controls }\end{array}$ \\
\hline $\begin{array}{l}\text { Betancourt et } \\
\text { al., } 2010\end{array}$ & rat & $\begin{array}{l}25, \quad 250 \mu g / k g \\
\text { b.w. orally }\end{array}$ & $\begin{array}{ll}\text { GD } & 10 \text { to } \\
\text { GD21 }\end{array}$ & $\begin{array}{l}\text { additional groups } \\
\text { received DMBA; } \\
30 \mathrm{mg} / \mathrm{kg} \mathrm{b.w..} \\
\text { on PND 50, or } \\
\text { PND100. }\end{array}$ & $\begin{array}{l}\text { 1. no DMBA treatment: } \\
\text { increased cell proliferation } \\
\text { 2. DMBA treatment only on day } 100 \text { but not on day } 50 \text { : } \\
\text { increased tumor incidence while decreasing tumor latency }\end{array}$ \\
\hline $\begin{array}{l}\text { Jones et al., } \\
2010\end{array}$ & $\begin{array}{l}\text { Brca1 } \\
\text { knockout }\end{array}$ & $\begin{array}{l}0.000250 \\
\text { continously sc }\end{array}$ & 4 weeks & & increased mammary epithelial cell proliferation and hyperplasia \\
\hline
\end{tabular}


The safety of the use of bisphenol A in medical devices

\begin{tabular}{|c|c|c|c|c|c|}
\hline & mouse & & & & \\
\hline $\begin{array}{l}\text { Jenkins et al., } \\
2011\end{array}$ & \begin{tabular}{|l} 
transgenic \\
MMTV-erbB2 \\
mammary \\
tumour \\
mouse
\end{tabular} & $\begin{array}{l}0.0005,0.005 \\
0.050,0.5 \\
\text { orally }\end{array}$ & PND 56-252 & & decreased tumour latency and increased tumour multiplicity \\
\hline $\begin{array}{l}\text { Weber Lozada } \\
\text { and Keri (2011) }\end{array}$ & mouse & $\begin{array}{l}0.025,0.250 \text { by } \\
\text { gavage }\end{array}$ & GD8-21 & DMBA & reduction of tumour latency of mammary tumours \\
\hline $\begin{array}{l}\text { Kass et al., } \\
2012\end{array}$ & rat & $\begin{array}{l}0.7,64 \mu \mathrm{g} / \mathrm{kg} \\
\text { b.w./day }\end{array}$ & $\begin{array}{l}\text { GD9 until } \\
\text { weaning in F0 }\end{array}$ & & $\begin{array}{l}\text { In F1 on GD21 but not on day } 18 \text { delayed histological MG } \\
\text { differentiation }\end{array}$ \\
\hline $\begin{array}{l}\text { Tharp et al., } \\
2012\end{array}$ & $\begin{array}{l}\text { rhesus } \\
\text { monkeys }\end{array}$ & 0.400 orally & GD 100-165 & & $\begin{array}{l}\text { statistically significant difference in the number of mammary } \\
\text { buds/ductal units }\end{array}$ \\
\hline $\begin{array}{l}\text { Vandenberg et } \\
\text { al., } 2013\end{array}$ & mice & $\begin{array}{l}0.25,2.5,25 \text { or } \\
250 \mu \mathrm{g} / \mathrm{b} . \mathrm{w} . / \text { day } \\
\text { via osmotic mini- } \\
\text { pumps }\end{array}$ & GD8-PND16 & & $\begin{array}{l}\text { advanced gland development at } 0.25 \text { and } 2.5 \mu \mathrm{g} / \mathrm{kg} \mathrm{b} . \mathrm{w} \text {. but } \\
\text { not at higher doses }\end{array}$ \\
\hline $\begin{array}{l}\text { US FDA/NCTR, } \\
2013 \text { / Delclos } \\
\text { et al., } 2014\end{array}$ & rats & $\begin{array}{l}2.5,8,25,80, \\
260,840,2700, \\
100000 \\
300000 \mu g \\
\text { BPA/kg b.w./day } \\
\text { by gavage }\end{array}$ & $\begin{array}{l}\text { dams: GD } 6 \\
\text { up to labour } \\
\text { onset } \\
\text { pups: PND } 1 \\
\text { until tissue } \\
\text { harvesting, } \\
\text { up to PND } 90\end{array}$ & & LOAEL for ductal hyperplasia $2,700 \mu \mathrm{g} / \mathrm{kg}$ bw \\
\hline
\end{tabular}




\section{General tumorigenicity}

In a study, carcinogenicity (including thyroid and lungs) was induced by N-nitrosobis-(2hydroxypropyl)-amine (BHP) (Takashima et al., 2001). The effect of BPA exposure during development in this model was investigated giving oral doses of 0 or $10,000 \mathrm{ppm}(\mathrm{mg} / \mathrm{kg}$ in feed) BPA for 10 weeks prior to mating, and through mating, gestation and lactation. Intakes of BPA were reported to be about $400-600 \mathrm{mg} / \mathrm{kg}$ b.w./day. Beginning at 5 weeks of age and continuing for 12 weeks, offspring in each group received either tap water or tap water containing 2000 ppm (mg/L) BHP. Dam body weight was lower in the BPA group compared to the control group during the gestation period and at weaning. Otherwise, BPA had no effect on food intake and maternal serum levels of triiodothyronine, thyroxine, and thyroid-stimulating hormone, weights or histopathological alterations of maternal organs, including uterus and ovary, on mating, fertility, duration of gestation, live-born pups, implantation loss, or offspring viability through PND 21. In pups from dams exposed to BPA compared to pups from control dams, body weights were higher (by $11 \%$ ) in females at 3 days of age and lower in males and females at 10 days and 2 weeks of age (16-22 \% decreases in males and 12$19 \%$ decreases in females). Prenatal and postnatal BPA exposure was not associated with significant differences in the development of BHP-induced neoplasms in the offspring (Takashima et al., 2001). The results of this study indicate that oral exposure to 400-600 $\mathrm{mg} / \mathrm{kg}$ b.w./day BPA during development does not exert promoting effects on BHPinduced thyroid, lung, liver, thymus and esophagus carcinogenesis in rats.

Doses of $0,0.05,7.5,30$, and $120 \mathrm{mg} \mathrm{BPA} / \mathrm{kg} \mathrm{b.w./day} \mathrm{were} \mathrm{administered} \mathrm{by} \mathrm{gavage} \mathrm{to}$ female F344 rats during pregnancy and lactation (Ichihara al., 2003). At 5 weeks of age, 21 male rats/group were injected subcutaneously with $50 \mathrm{mg} / \mathrm{kg} \mathrm{b.w./day} \mathrm{3,2-dimethyl-}$ 4-aminobiphenyl (DMAB) 10 times at 2-week intervals. DMAB is an experimental aromatic amine that serves as an experimental model for arylamine and heterocyclic amine mutagens (Ravoori et al., 2008). There were no consistent or dose-related effects on multiple endpoints. Without DMBA treatment, incidences of prostatic intraepithelial neoplasia (PIN), carcinoma, and atypical hyperplasia were not increased by exposure to BPA, and there were no increases in tumours of the non-reproductive organs. No effect was observed on serum testosterone levels. This screening study showed that exposure of rat dams up to $120 \mathrm{mg} / \mathrm{kg} \mathrm{b.w./day} \mathrm{BPA} \mathrm{during} \mathrm{the} \mathrm{gestation} \mathrm{and} \mathrm{lactation} \mathrm{periods} \mathrm{did}$ not predispose their offspring to prostate cancer development later on in life (65 weeks of age).

Effects of maternal exposure to BPA on uterine carcinogenesis were studied in offspring of Donryu rats (a strain of rats with a high rate of spontaneous and ENNG-induced uterine tumours) administered BPA (0, 0.006 and $6 \mathrm{mg} / \mathrm{kg} \mathrm{b.w./day,} \mathrm{n}=12,15$ and 19/group, respectively) daily by gavage from GD 2 to PND 21 (Yoshida et al., 2004). At 11 weeks of age, 35 - 36 animals/group were injected in the uterine horn with N-ethyl$\mathrm{N}^{\prime}$-nitro-nitrosoguanidine (ENNG) to initiate uterine carcinogenesis. About 24 weeks later, the uteri of the $24-30$ surviving animals/group were examined histologically to detect tumours and other lesions. In dams exposed to BPA, there were no clinical signs of toxicity or effects on body weight, implantation sites, or gestation length and on litter size. BPA exposure had no effect on the pups. The incidence of uterine preneoplastic or neoplastic lesions induced by ENNG was not increased by BPA exposure.

\section{Prostate}

The effect of short-term neonatal exposure to BPA on susceptibility of Sprague Dawley rats to prostate cancer was investigated after s.c. injection of BPA on PND 1, 3 and 5. The dose administered was $10 \mu \mathrm{g} / \mathrm{kg}$ b.w.s.c. corresponding to $41.8 \mu \mathrm{g} / \mathrm{kg}$ b.w.by the oral route that was based on AUCs after oral and s.c. administration allowing the calculation of anoral systemic availability of $23.9 \%$ (Ho et al., 2006). From PND 90, in $50 \%$ of the rats of every group $17 \beta$-estradiol $(E)$ and testosterone $(T)$ were administered by s.c. implantation for 16 weeks in a dose which is reported to induce prostatic intraepithelial neoplasia (PIN) in $33 \%$ of Sprague Dawley rats. The second half of rats served as control. At 28 weeks, BPA exposure alone showed no effects on dorsal prostate 
weight, histopathology alterations, proliferation index, or apoptotic index. In animals that were given $E$ and T simultaneously for 16 weeks from PND 90, the group of rats with BPA exposure showed a statistically significant increased incidence and severity of PIN (100 [10/10] vs. $40 \%$ [4/10] incidence in controls). In the BPA/E+T group compared to the $\mathrm{E}+\mathrm{T}$ group, the proliferation index was increased and the apoptosis index was decreased in regions where PIN was observed. Furthermore, the authors found hypomethylation of the PDE4 gene and increased expression of that gene at 90 and 200 days of age, with or without $\mathrm{E}+\mathrm{T}$ exposure in adulthood.

A study by the same group (Prins et al., 2011) used the identical protocol with the modification that a group with oral dosing was included whereby s.c. and oral dosing was at the same level, namely $10 \mu \mathrm{g} / \mathrm{kg}$ b.w.. Kinetic studies revealed an oral systemic availability of $23.9 \%$ of the dose. The study confirmed the findings of the earlier study. Notably, identical effects were elicited by s.c. and oral dosing, although the internal dose after oral dosing was about $25 \%$ of the s.c. dose. Thus, no dose-response relationship could be demonstrated.

In a mechanistic study, the group of Prins (Tang et al., 2012) evaluated methylation and expression of several genes throughout life in the rat prostate gland by BPA given by s.c. administration in three doses of $10 \mu \mathrm{g} / \mathrm{kg} \mathrm{b.w}$. (corresponding to $36 \mu \mathrm{g} / \mathrm{kg}$ each) each on PND 1, 3, and 5 with a low $(0.1 \mu \mathrm{g} / \mathrm{kg}$ b.w.) and a high $(2500 \mu \mathrm{g} / \mathrm{kg} \mathrm{b.w.)}$ dose of $17 ß-$ estradiol-3-benzoate (EB) as controls. Furthermore, a group of the treated rats received additional treatment, with estradiol (E) plus testosterone $(T)$ released via a s.c. implanted capsule to produce increased intraepithelial neoplasias in the prostate, a model which has been used by the group since 1981 (Lee et al., 1981). Further results were obtained in prostate cell lines. A whole array of changes were observed in vivo: hypomethylation of the promoter of nucleosom binding protein-1 persisting until day 100; hypermethylation of hippocalcin-like1 which shows changes throughout life; persistent overexpression of four of eight genes functioning in methylation/demethylaton of DNA not related to DNA methylation at their promotors. The results are somewhat inconclusive because a) no dose dependency was observed for the treatment with EB the dose differing by a factor of $25,000 \mathrm{~b}$ ) no clear difference existed in the parameters for the group undergoing additional treatment with $\mathrm{E}+\mathrm{T}$ and $\mathrm{c}$ ) more than 50 statistical tests were performed when testing the in vivo results. Although the authors claim that Bonferroni posthoc test was performed to correct for multiple testing, it is uncertain to which data it was applied.

\section{Mammary gland effects}

The effect of BPA was investigated in the N-nitroso- $\mathrm{N}$-methylurea (NMU) model for inducing mammary tumors in Wistar rats (Durando et al., 2007). On gestational day (GD) $8-23$, s.c. (via miniature osmotic pumps) doses of 0 or $0.025 \mathrm{mg} / \mathrm{kg} \mathrm{b.w./day}$ (corresponding to $0.9 \mathrm{mg} / \mathrm{kg} \mathrm{b} . \mathrm{w}$. day on the oral route) BPA were given. Offspring were killed before puberty (PND 30), after puberty (PND 50), or in adulthood (PND 110 and 180). In mammary gland stroma and epithelium proliferation, apoptotic cells were determined and morphometric analyses were performed using adequate methods. Part of the offspring was examined for responsiveness towards the established carcinogen $\mathrm{N}$ nitroso-N-methylurea (NMU). On PND 50, NMU was administered intraperitoneally to 1016 offspring from the vehicle control group at 25 or $50 \mathrm{mg} / \mathrm{kg} \mathrm{b.w}$. and to 21 offspring from the BPA group at $25 \mathrm{mg} / \mathrm{kg}$ b.w./day. Based on findings from a pilot study, 25 $\mathrm{mg} / \mathrm{kg}$ b.w. NMU was considered a sub-carcinogenic dose and $50 \mathrm{mg} / \mathrm{kg} \mathrm{b} . \mathrm{w}$. NMU was considered a positive control.

Anogenital distance on PND 1 or 5 and postnatal body weights were unaffected in pups exposed to BPA. Vaginal opening was 5 days earlier in pups exposed in intra-uterine life to subcutanous BPA (mean PND 34 to PND 39 in controls). On PND 50, the BrdU/apoptosis ratio was significantly increased and apoptosis was significantly decreased in mammary parenchyma and stroma of BPA-exposed animals; the effects were not observed on PND 30 or PND 110. Significantly increased percentages of hyperplastic ducts, density of stromal nuclei, and numbers of mast cells were observed in 
the BPA group on PND 110 and PND 180. In rats exposed to $25 \mathrm{mg} / \mathrm{kg} \mathrm{b.w./day} \mathrm{NMU} \mathrm{on}$ PND 50, incidence of hyperplastic lesions on PND 180 was significantly higher in the group with prenatal BPA exposure compared to controls (mean incidence of $35.5 \%$ compared to $15.7 \%$ in controls). Other results were not statistically significant. As no dose-response relationship was investigated, the results on the mammary gland cell proliferation should be considered as supporting evidence and indicator for a possible concern.

Murray et al. (2007) studied whether prenatal BPA exposure induced mammary tumours in rats. Wistar-Furth rat dams were exposed via subcutaneously implanted osmotic pumps to BPA $0,0.0025,0.025,0.250$, or $1 \mathrm{mg} / \mathrm{kg}$ b.w./day from GD 9 through PND 1. Vehicle control exposure was 50\% DMSO.

The number of hyperplastic ducts was increased in all dose groups on PND 50; the study authors noted that the effect on PND 50 was quantitatively similar in all dose groups (i.e. 3-4-fold increase) lacking a dose-response. Some of the hyperplasias were classified as carcinoma in situ with cribriform structures. These were observed in the 0.25 (corresponding to $9 \mathrm{mg} / \mathrm{kg} / \mathrm{d}$ oral dosing) and 1 (corresponding to $35.7 \mathrm{mg} / \mathrm{kg} / \mathrm{d}$ oral dosing) $\mathrm{mg} / \mathrm{kg} \mathrm{b.w./day} \mathrm{groups.} \mathrm{The} \mathrm{incidence} \mathrm{was} \mathrm{25 \%} \mathrm{(1} \mathrm{out} \mathrm{of} 4$ ) at PND50 and 33\% (2 out of 6 ) for the two highest dosed groups $(0.25$ and $1 \mathrm{mg} / \mathrm{kg})$. The study authors concluded that fetal BPA exposure at dose levels of 0.250 and $1 \mathrm{mg} / \mathrm{kg} \mathrm{b.w./day} \mathrm{via}$ subcutaneously implanted minipumps (corresponding to oral doses of $9 \mathrm{mg} / \mathrm{kg} \mathrm{b} . \mathrm{w}$./day and $36 \mathrm{mg} / \mathrm{kg} \mathrm{b.w./day)} \mathrm{is} \mathrm{able} \mathrm{to} \mathrm{induce} \mathrm{development} \mathrm{of} \mathrm{preneoplastic} \mathrm{and} \mathrm{neoplastic}$ mammary lesions in rats. There were some limitations to this study. Background diet, drinking water, bedding, and cage provided "negligible" estrogenicity. The forming of ductal end buds usually appears at puberty (Lucas et al., 2007). Bud formation and ductal hyperplasia might be seen as an adverse outcome or as part of the normal ductal development depending on the status of the animals in terms of mammary gland develoment. A positive control like estradiol, lacking is this study, is then needed for comparison and identification of the adverse outcome. It is unclear whether these findings are biologically related to carcinogenic hazard. However, the results show a difference clearly induced by BPA and confirm earlier observations of this research group for the possibility for an effect on the developing mammary gland (Durando et al., 2007).

The same research group used the same exposure protocol (s.c. osmotic pumps, dosing $0.25,2.5,25 \mu \mathrm{g} / \mathrm{kg}$ b.w) for long-term evaluation of mammary gland alterations in mice (Vandenberg et al., 2008). Mice were exposed from gestation day 8 until day 16 of lactation. At 3, 9 and $12-15$ months of age female offspring were killed and mammary tissue samples collected and evaluated. An increase in the volume fraction of alveolar buds in the mammary tissue was observed for the $0.25 \mu \mathrm{g} / \mathrm{kg} \mathrm{b.w.} \mathrm{BPA} \mathrm{group} \mathrm{only,} \mathrm{at}$ month 3 and 9. A dose-response relationship was not present. At 9 months, an increase in the incidence of beaded ducts was also noted for all doses investigated, although a dose-response relationship was not present. At month $12-15$, the incidence of beaded ducts was increased only for the lowest dose. Proliferation of the cells was indicated by the Ki-67 antigen staining. Histological analysis of mammary glands at 9 months was unremarkable as to periductal stroma width, periductal collagen density, proliferative index (Ki-67 staining) and \% ER and \% AR positive cells.

BPA exposure during pregnancy and lactation affected the development of the mammary glands in the exposed female offspring. It is unclear whether these findings are biologically related to a carcinogenic hazard. No dose-response was observed and the authors interpreted the results as indicating non-monotonicity.

In a study by Moral et al. (2008), pregnant rats were given $25 \mu \mathrm{g} \mathrm{BPA} / \mathrm{kg} \mathrm{b.w.} \mathrm{or} 250 \mu \mathrm{g}$ $\mathrm{BPA} / \mathrm{kg}$ b.w. from GD 10 to GD21. Female litters were euthanized at $21,35,50$, and 100 days. Analysis of mammary gland morphology was performed from whole-mounted mammary tissue. Proliferative index was determined by quantifying bromodeoxyuridine incorporation in the epithelial cells. BPA exposure induced changes in the mammary gland that were time and dose specific. High-dose exposure resulted in increased number of undifferentiated epithelial structures of the breast tissue. Proliferative index did not 
show an effect of BPA. The study results are well described. However, it remains unclear what the findings indicate.

Neonatal/prepubertal rats were exposed to BPA via lactation from nursing dams treated orally with 0,25 , and $250 \mu \mathrm{g} \mathrm{BPA} / \mathrm{kg}$ body weight/day from PND 1 to PND 21 (Jenkins et al., 2009). In addition, female offspring were exposed to $30 \mathrm{mg} \mathrm{DMBA} / \mathrm{kg}$ body weight at 50 days of age. Lactational BPA exposure resulted in increased cell proliferation and decreased apoptosis at 50 but not 21 days PND. This means that no effect was seen at the end of exposure. When additional DMBA treatment has been performed, lactational exposure to BPA demonstrated a dose-dependent increase in mammary tumor multiplicity and reduced tumor latency. The effect of DMBA is similar as seen in other studies. It is, however, not clear whether the findings indicate a carcinogenic hazard.

A similar protocol was used in the study of Betancourt et al., 2010. Oral treatment with 0,25 or $250 \mu \mathrm{g} \mathrm{BPA} / \mathrm{kg}$ b.w. was given to pregnant rats from GD 10 to GD21. For tumorigenesis experiments, prenatally exposed female offspring received a single dose of $30 \mathrm{mg} / \mathrm{kg}$ b.w. DMBA by gavage on PND 50, or PND 100. Prenatal exposure of the dam to $250 \mu \mathrm{g} \mathrm{BPA} / \mathrm{kg}$ b.w. increased cell proliferation. Prenatal exposure of the dam to 250 $\mu \mathrm{g} \mathrm{BPA} / \mathrm{kg} \mathrm{b.w.} \mathrm{combined} \mathrm{with} \mathrm{a} \mathrm{single} \mathrm{exposure} \mathrm{of} \mathrm{female} \mathrm{offspring} \mathrm{to} \mathrm{DMBA} \mathrm{had} \mathrm{an}$ effect only when dosed on PND 100, but not when dosed on PND 50. In those animals, tumor incidence increased significantly and tumor latency was decreased in comparison to the control group. The effect of DMBA is similar as seen in other studies. It is, however, not clear whether the findings indicate a carcinogenic hazard.

Three studies originating from the research group of Soto et al. demonstrated an effect of prenatal BPA exposure on mammary gland development, i.e. ductal hyperplasia and in one study carcinoma in situ development. These studies were performed with very low background estrogen levels in the feed that may have had an effect on the normal development in the controls. A limitation is the lacking of a positive control like estradiol for comparing the estrogenic effects induced by BPA. However, the differences between the non-treated and prenatal/postnatal BPA exposed animals are clear, so it cannot be excluded that BPA affects early development of mammary tissue. Therefore, these studies should be considered as an indicator for a possible concern.

Recently, Ayyanan et al. (2011) also showed that perinatal exposure of mice to BPA at doses ranging from $1.2 \mu \mathrm{g}$ to $1.2 \mathrm{mg} \mathrm{BPA} / \mathrm{kg} \mathrm{b.w./day} \mathrm{and} \mathrm{DES} \mathrm{at} 0.12$ and $1.2 \mu \mathrm{g} / \mathrm{kg}$ b.w./day via drinking water. Exposure to low doses of oral BPA had no significant effect on litter size, sex ratio, or body weight at weaning. The number of terminal end buds, estrogen-induced proliferative structures, was altered in a dose-dependent fashion, but for only one dose ( $3 \mu \mathrm{g} / \mathrm{kg}$ b.w./day), an increase was suggested. In addition, adult F1 females showed an increase in mammary epithelial cell numbers at three months of age for both BPA (low $6-12 \mu \mathrm{g} / \mathrm{kg}$ and high $600-1200 \mu \mathrm{g} / \mathrm{kg}$ intake) and DES exposed F1 females. However, the study reported effects on increasing adjusted number of buds at a dose of $0.6 \mu \mathrm{g} / \mathrm{kg} \mathrm{b.w./day.} \mathrm{As} \mathrm{the} \mathrm{paper} \mathrm{does} \mathrm{not} \mathrm{clearly} \mathrm{describe} \mathrm{the} \mathrm{procedure} \mathrm{how}$ the number of buds was adjusted and because the dose is really unclear (differences between method section and results section differ up to a factor of 1000), the study cannot be considered valid.

More recently, Tharp et al. (2012) of the group of Soto, investigated the histopathology of mammary gland in the offspring of rhesus monkeys given orally $400 \mu \mathrm{g}$ of BPA per $\mathrm{kg}$ of body weight daily from gestational day 100 to term. This regimen resulted in $0.68 \pm$ $0.312 \mathrm{ng} / \mathrm{mL}$ of free BPA and $39.09 \pm 15.71 \mathrm{ng} / \mathrm{mL}$ of conjugated BPA in serum measured after dosing with deuterated BPA. The serum concentration is about 250 times higher than the predicted concentration in humans at a high realistic dose of $1 \mu \mathrm{g} / \mathrm{kg} \mathrm{b} . \mathrm{w} . / \mathrm{day}$ by the oral route. Morphometric analysis of the mammary glands removed from female offspring at birth showed that only the density of mammary buds was significantly increased in BPA exposed monkeys. Other parameters like total area, ductal area, number of ducts, and terminal ends showed no difference between BPA exposed and 
control animals. In general the development of their mammary gland seemed more advanced when compared to unexposed monkeys.

BPA induced proliferative changes in the mammary gland of male CD-1 mice when BPA was given to pregnant and lactating mice at doses of $0.25,2.5,25$ or $250 \mu \mathrm{g} / \mathrm{b} . \mathrm{w} . /$ day via osmotic mini-pumps (Vandenberg et al., 2013). In this study, mammary glands were examined at $3-4,7-9$ and $12-16$ months in the adult male offspring. The reported changes were seen in the ductal area and at branching points. Animals exposed to 0.25 or $2.5 \mu \mathrm{g} / \mathrm{kg}$ b.w./day showed more advanced gland development than the controls, whereas animals having dosed with 25 or $250 \mu \mathrm{g} / \mathrm{kg}$ b.w./day had no statistically different results compared with controls. These results point at a non- monotonic doseresponse to BPA. At later time periods, effects were similar. However, the dose-response relationship had a different pattern.

Jones et al. (2010) used the Brca1 knockout mouse model of breast cancer susceptibility (gene 1 (BRCA1) related mammary cancer). Continuous exposure to $250 \mathrm{ng} / \mathrm{kg}$ b.w. BPA through an osmotic pump for 4 weeks (corresponding to $125 \mu \mathrm{g} / \mathrm{kg} \mathrm{b.w./day} \mathrm{by} \mathrm{the} \mathrm{oral}$ route) increased mammary epithelial cell proliferation and hyperplasia in adult Brca1 knockout mouse mammary glands compared with wild type mice. The authors also presented in vitro mechanistic investigations in MCF-7 cells supporting the hypothesis that loss of BRCA1 function in mammary cells would enhance BPA-induced cell proliferation via interference with the ER-alpha signalling pathway.

Jenkins et al. (2011) investigated in a transgenic MMTV-erbB2 mammary tumour mouse model, whether BPA increased the susceptibility of females to mammary cancer after chronic oral exposure to BPA at levels of $0,2.5,25,250,2500 \mu \mathrm{g}$ BPA/L in drinking water for the whole adult life (PND 56-252). In this model, BPA decreased tumour latency and increased tumour multiplicity, enhanced tumour volume and higher incidence of lung metastasis in a way that the authors describe a non-monotonic dose-response as the effects were observed at one of the two lower doses $(0.5$ and/or $5 \mu \mathrm{g} B P A / \mathrm{kg}$ b.w./day), but not at 50 or $500 \mu \mathrm{g} \mathrm{BPA} / \mathrm{kg}$ b.w./day. In contrast, the cell proliferation index of mammary epithelial cells (evaluated on PND 112) and the apoptotic index increased in a dose-dependent manner, with statistical significant results at the highest dose (500 $\mu \mathrm{g} \mathrm{BPA} / \mathrm{kg}$ b.w./day).

Similar to the study of Ichihara al., 2003, Weber Lozada and Keri (2011) used the DMBA mammary tumour mouse model to assess the effects of foetal exposure to BPA on mammary tumour development in adults. When mice were exposed in utero to $25 \mu \mathrm{g} / \mathrm{kg}$ b.w. and $250 \mu \mathrm{g} / \mathrm{kg}$ b.w. by oral gavage of the pregnant dams, the offspring showed an increased susceptibility to DMBA mammary gland induction when treated with DMBA postnatally. A dose-response in the reduction of tumour latency of mammary tumours was observed in mice treated with BPA before birth. The foetal exposure to BPA led to early vaginal opening in FVB/N female mice. In these studies, the mouse strain $\mathrm{FVB} / \mathrm{N}$ was used because of its intrinsic propensity to develop mammary tumors with various genetic manipulations. The administration of BPA only had no effect on mammary gland development. BPA was administered from postcoital day 8 until birth, while DMBA was administered twice one dose each at week 5 and 6 after birth. Additionally, tumour growth promotion was observed for both BPA and $17 \beta$-oestradiol treated mice after injection of estrogen dependent MCF-7 human breast cancer cells in ovariectomized nude mice. However, the effect of BPA was reduced when compared to $17 \beta$-oestradiol.

Kass et al. (2012) found in F1 bred female offspring of BPA and DES treated dams a delay in histological mammary gland differentiation and altered milk yield pattern during lactation. BPA with theoretical doses of $0.5 \mu \mathrm{g} \mathrm{BPA} / \mathrm{kg}$ b.w./day and $50 \mu \mathrm{g} B P A / \mathrm{kg}$ b.w./day was administered via the drinking water from GD9 until PND21. Direct exposure to BPA or DES in the drinking water did not produce signs of embryotoxicity (i.e. all pregnant dams successfully delivered their pups, and the number of live-born pups per litter was similar among groups), abnormal maternal or nursing behavior, or changes in body weight gain in the FO dams. The reproductive parameters were not significantly 
changedin the $\mathrm{F} 1$ females, with the exception of the number of resorption sites in BPA 50 $\mu \mathrm{g} / \mathrm{kg}$ b.w. and DES treated dams. The number of pups born of the F1 females was decreased, but did not reach significance.

Acevedo et al. (2013) investigated effects of subcutaneously administered BPA in female rats during gestation and throughout lactation on mammary gland development in female offspring. Atypical ductal hyperplasia $(A D H)$ was reported in a few animals in all treatment groups without reaching statistical significance. In addition, in several treatment groups adenocarcinomas were observed in individual animals. However, the incidence was not increased when compared to control animals.

Delclos et al. (2014) published the results of a toxicity study of BPA using a low dose range $(2.5-2700 \mu \mathrm{g} / \mathrm{kg}$ b.w./day, with a total of 7 doses within this range) below the current NOAEL of $5,000 \mu \mathrm{g} / \mathrm{kg}$ b.w./day and two very high $(100,000-300,0000 \mu \mathrm{g} / \mathrm{kg}$ b.w./day) doses of BPA. The effects of BPA were compared to ethinyl estradiol (EE2) (0.5 and $5.0 \mu \mathrm{g} / \mathrm{kg}$ b.w./day) to evaluate estrogen responsiveness. On PND 21 mammary gland ductal hyperplasia was observed in females in two low dose BPA groups (25 and $2700 \mu \mathrm{g} / \mathrm{kg}$ b.w./day, respectively), and the $300,000 \mu \mathrm{g} / \mathrm{kg} \mathrm{b.w./day} \mathrm{BPA} \mathrm{dose} \mathrm{group.}$ There was no clear dose response effect observed. When severity was also included in the evaluation for 2700 and $100,000 \mu \mathrm{g} / \mathrm{kg}$ b.w. day, an increase of mammary gland hyperplasia was noted. The incidence of hyperplastic lesions was statistically significant by at least one of the three statistical methods used when compared with the vehicle control group in the 2,700 and $100,000 \mu \mathrm{g} / \mathrm{kg}$ bw per day groups, but not in the 300,000 $\mu \mathrm{g} / \mathrm{kg}$ bw per day group. This observation was considered possibly treatment-related by the study authors but not by the original study pathologist.

\section{Leydig cell division}

In a study in which pregnant and lactating Long-Evans rats were given BPA via gavage ( 2.5 and $25 \mu \mathrm{g} / \mathrm{kg}$ b.w./day) from gestational day 12 to postpartum day 21 , Leydig cell division was stimulated in the prepubertal period and increased Leydig cell numbers were shown in the testes of adult male rats at 90 days (Nanjappa et al., 2012).

\section{Conclusion on BPA carcinogenicity}

From all the study results taken together, it can be concluded that in standard carcinogenic testing protocols according to OECD, BPA has no carcinogenic activity. In addition, in multigeneration studies, (Tyl et al., 2002; Tyl et al., 2008) no indication of increased cancerogenicity was observed; in particular, pre-neoplatic lesions of the mammary gland were absent in all offspring. In contrast, several studies in rats, using s.c. exposure via osmotic pumps, demonstrated an effect of prenatal BPA exposure on mammary gland development, i.e. ductal hyperplasia, and in one study carcinoma development in situ. These studies were performed with low background estrogen levels in the feed that may have had an effect on the normal development in the controls. A limitation is the lack of a positive control like estradiol for comparing the estrogenic effects induced by BPA. Similar effects were indicated in studies in mice and rhesus monkeys, supporting the observations in rats. The differences observed between the non-treated and prenatal/postnatal BPA exposed animals is clear, so this effect cannot be excluded. These studies should be considered as an indicator for a possible concern, although the relevance for humans is not clear.

Studies using s.c. administration of BPA indicated that BPA may have the ability to increase the effects of well-known carcinogens even at low BPA levels, acting as promotor. The studies had limitations which render them unsuitable for assessing whether BPA has such an effect following prenatal or peri-natal exposure. The main limitation is that in the studies with positive outcome additional treatment with a strong initiating or additional promoting agent(s) has been performed. Furthermore, in most of the studies multiple statistical testing has been performed without proper adjustment to 
avoid positive results by chance. An additional problem in the statistical analysis is the lack of considering litter effects.

Further studies were performed in transgenic animals, the results of which cannot be extrapolated directly to humans.

In conclusion, the studies indicating effects on mammary gland, i.e mammary gland ductal hyperplasia in female rats raise some concern for a possible effect after prenatal exposure to BPA given similar findings in other studies. EFSA assessed the mammary ductal gland hyperplasia as relevant for the risk assessment of BPA (EFSA 2015). However, EFSA stated that the analysis on the mammary gland data revealed large differences in the benchmark dose estimates obtained with the various models and wide intervals were obtained with some of the models (EFSA 2015). Therefore, the general toxicity, specifically the effects on relative kidney weight in a two-generation study in mice, was used for calculation of the BMDL ${ }_{10}$.

\subsubsection{Neurotoxicity and behavioural toxicity}

Neurological studies in laboratory animals (rat, mouse, sheep and/or non-human primate) assayed pathology, neurochemistry, neuroendocrine system, sensory systems, locomotor and spontaneous activity, social and sexual behaviours, anxiety, and learning and memory at various stages of development. Exposure was primarily during the periods of gestation and lactation.

The experimental evidence does not support brain developmental neuropathological changes (e.g. cortical thickness, cerebellum height, height of hippocampal layers) at rat maternal dietary exposures below $164 \mathrm{mg} / \mathrm{kg}$ b.w./day (Stump et al., 2010). Brain biochemical changes (e.g. monoaminergic, cholinergic, glutamatergic, nuclear receptor expression and signalling) were reported in rodents at dietary exposures below $5 \mathrm{mg} / \mathrm{kg}$ b.w./day. However, no additional functional testing was performed. Thus, the in vivo consequences of the changes are unclear.

Only a few studies have specifically focused on the impact of BPA on morphometric and cellular brain sex differences. Depending on the hypothesized mode of action, not all studies included both sexes. In some cases, only one sex was impacted, whereas in others, the overall differences between the sexes were reduced or eliminated. The controversial results limit their interpretation (reviewed in Wolstenholme et al. 2011,).

BPA does not appear to affect sensory systems, spontaneous activity or female sexual behaviour in rodents. For neonatal reflexes, sensory response, spontaneous motor activity and other open field behaviours, a minimum NOAEL of $164 \mathrm{mg} / \mathrm{kg} \mathrm{b} . \mathrm{w} . /$ day for rat maternal dietary exposure can be identified. Minimum NOAELs (corresponding to the highest dose tested in individual studies) of $200 \mu \mathrm{g} / \mathrm{kg} \mathrm{b.w}$. per day (Ryan et al., 2010) and $320 \mathrm{mg} / \mathrm{kg} \mathrm{b.w./day} \mathrm{(Kwon} \mathrm{et} \mathrm{al.,} \mathrm{2000)} \mathrm{for} \mathrm{rodent} \mathrm{maternal} \mathrm{dietary} \mathrm{exposure} \mathrm{could}$ be identified for lordosis; for other components of sexual/sociosexual behaviours, NOAELs could not be identified. For learning and memory in rodents, conflicting data exist, although the weight of evidence does not suggest these to be a concerning hazard identification end-point (Kwon et al., 2000).

Neuroendocrine data in rodents and sheep suggest effects on female hypothalamicpituary-gonadal (HPG) axis organization ( $\geq 50 \mu \mathrm{g} / \mathrm{kg}$ b.w. per day, non-oral route) and function ( $\geq 5 \mathrm{mg} / \mathrm{kg}$ b.w./day, non-oral route), that is at doses higher than the Point of Departure used for TDI derivation (Katoh et al., 2004). The specific mechanisms by which this occurs remain to be identified, but some data suggest that the pattern of luteinizing hormone release may be altered by exposure, resulting in blunted secretion and resistance to feedback. 
Recent studies (Ishido et al., 2011; Kim et al., 2011; Wolstenholme et al., 2011; EilamStock et al., 2012) have examined the effects of BPA at doses lower than $50 \mu \mathrm{g} / \mathrm{kg}$ b.w.. The endpoints were neuroanatomical/genomic and behavioural.

The study by Wolstenholme et al. in 2011 investigated the effect of dietary exposure towards BPA ( $5 \mu \mathrm{g} / \mathrm{kg}$ b.w./day) on social interactions in the pups and also some gene expressions. The female offspring showed increased social interactions in a free $30-\mathrm{min}$ social interaction test. However, BPA did not affect social preference for the stimulus animal in a social preference test. In the Plus Maze task, anxiety, time spent in the open arms, closed arms and the number of crosses between arms were similar in the two groups. Gene expression analysis revealed that mRNA for the glutamate transporter Slc1a1 was enhanced by exposure to BPA in female brains and that expression of two of the three DNA methyltransferase genes, Dnmt1 and Dnmt3a, was modulated by BPA. Whereas expression of estrogen receptors' genes was not affected by BPA, oxytocin receptor gene was to some extent reduced in males. Although the association of the behavioural results at weaning age with the small changes in gene expression found at the fetal stage is weak and do not fully support novel mechanistic hypotheses, these findings confirm previous data on the sex-dimorphic effects of BPA on social behaviour.

The effects of a single subcutaneous BPA administration ( $40 \mu \mathrm{g} / \mathrm{kg} \mathrm{b.w.)} \mathrm{on} \mathrm{memory} \mathrm{and}$ synaptic plasticity in adult male rats was examined by Eilam-Stock et al. (2012) in adult animals. Single subcutaneous administration of BPA interferes with memory consolidation possibly impairing the formation of dendritic spines by reducing a marker of neural plasticity and synaptic remodeling as PSD95 in the hippocampus. This study is remarkable as a single dose is sufficient to produce marked effects. However, notably, in rats, 40 and $80 \mu \mathrm{g} / \mathrm{kg} \mathrm{b.w.} \mathrm{given} \mathrm{s.c.} \mathrm{corresponds} \mathrm{to} \mathrm{roughly} 1$ and $2 \mathrm{mg} / \mathrm{kg} \mathrm{b.w}$. oral dosing, considering kinetic differences.

The study of Jones and Watson (2012) investigated the behaviour in the Morris Water Maze (MWM), Elevated Plus Maze (EPM) and Forced Swimming Test (FST) after oral administration of BPA in doses between $5 \mu \mathrm{g} / \mathrm{kg} \mathrm{b.w./day} \mathrm{and} 5000 \mu \mathrm{g} / \mathrm{kg} \mathrm{b.w./day}$ during gestation until PND 14 day to investigate their effects after delivery. No effect of BPA was observed in the MWM, but on both the EPM and FST, low and high doses (5 $\mu \mathrm{g} / \mathrm{kg}$ and $5000 \mu \mathrm{g} / \mathrm{kg}$ b.w./day) of BPA eliminated sex differences found between controls. However, interpretation of the result is difficult and might be due to statistical noise.

Three doses of $50 \mu \mathrm{g} / \mathrm{kg} \mathrm{b.w.} \mathrm{and} 50 \mathrm{mg} / \mathrm{kg}$ b.w. BPA each by subcutaneous injection (corresponding to an oral dose higher than $1 \mathrm{mg} / \mathrm{kg}$ b.w.) from PNDO to PND2 showed significant and sex-specific alterations of gene expression of estrogen receptor alpha $(\mathrm{ERa}), \mathrm{ER}$ beta $(\mathrm{ER} \beta)$ and kisspeptin (Kiss1) in the anterior and mediobasal hypothalamus on PND 4 and 10 of rats but not in other regions of the brain (Cao et al., 2012). The authors suggest that effects observed with BPA are very different from those of the positive control (oestradiol) and hence mechanisms were involved which are different from estrogenic action.

BPA induced locomotor hyperactivity when administered intracisternally at PND 5 in the brain of male rats (Ishido et al., 2004). A difference noted was that control animals received the vehicle olive oil only, while BPA treated rats received BPA dissolved in at least $50 \%$ ethanol complemented with olive oil. There was a dose dependent effect on the motor activity with a NOAEL of $0.02 \mu \mathrm{g} / \mathrm{pup}$ and a LOAEL of $0.2 \mu \mathrm{g} / \mathrm{pup}$, both administered as a single dose. The same group reported similar results for BPA in another study (Masuo et al., 2004). In an additional study only one dose was investigated (20 $\mathrm{\mu g} / \mathrm{pup}$ ) and BPA was compared with two of its derivatives (3hydroxybisphenol $A$, bisphenol A 3,4-quinone). With the same treatment modality (ethanol and olive oil), the derivatives did not induce a motor hyper activity whereas BPA did. So, the effects could not be attributed to the ethanol present in the administered solution (Ishido et al., 2011). The same protocol was applied in a study by Kiguchi et al., (2008) and the results the authors report are similar to the previous studies with the exception that the motor hyperactivity was observed in the light phase in contrast to the 
studies of Ishido et al., 2004, and Masuo et al., 2004, in which the hyperactivity was observed in the dark phase of the day. When comparing doses in the study of Kiguchi a higher dose was needed to induce the effect ( $40 \mu \mathrm{g}$ versus $0.2 \mu \mathrm{g}$ and $20 \mu \mathrm{g} / \mathrm{pup}$ ), the $20 \mu \mathrm{g}$ dose being ineffective (Kiguchi et al., 2008). The effect seems to be limited to a period directly after the administration, as at week 8-10 after the treatment no effect of the BPA administration on motor hyperactivity was observed (Kiguchi et al., 2007).

There are several studies published in the last three years investigating the effect of BPA in animal models for anxiety, for learning and memory, and for social behaviour.

\section{Anxiety}

Concerning anxiety, a number of studies have been done on rodents (e.g. Cox et al., 2010; Tian et al., 2010; Zhang et al., 2009; Patisaul and Bateman, 2008; Ryan and Vanderbergh, 2006; Gioiosa et al., 2007; Fujimoto et al., 2006). However, the results of these studies were controversial with either significant or not significant sex differences, which might be partly explained by the testing conditions used which were different in the studies and may have not exposed the animals in the window of susceptibility. The most recent studies (Matsuda et al., 2012; Jones and Watson, 2012; Patisaul et al., 2012; Jasarevic et al., 2012; Xu et al., 2012; Gioiosa et al., 2013; Kundakovic et al., 2013) assessed the effects of BPA on anxiety-like behaviour and brain biochemistry. The studies explored different exposure schedules and different doses.

Matsuda et al., 2012 showed that only in male offspring and not in females of C57BL/6] mice dosed from GD10 to PND20 to BPA $(0.25 \mu \mathrm{g} / \mathrm{kg}$ b.w./day subcutaneously) assessed at 4 weeks and at 8 weeks exhibited increased anxiety and dopamine concentrations and turnover in different brain areas were altered. In the rat study of Jones and Watson (2012) anxiety responses (Elevated Plus Maze (EPM); Forced Swimming Test (FST)) were measured after repeated oral exposure between GD 1 and PND 21 to BPA doses 5, 50, 500 , or $5000 \mu \mathrm{g} / \mathrm{kg} \mathrm{b.w./day.} \mathrm{Males} \mathrm{showed} \mathrm{greater} \mathrm{anxiety-like} \mathrm{behaviour} \mathrm{than} \mathrm{females}$ in the EPM and the lowest and the highest dose of BPA eliminated significant sex differences. In the FST test only for the lowest BPA dose eliminated sex differences. They also assessed spatial learning capacities in the Morris Water Maze and reported no effects of BPA or an interaction of BPA with sex. This study is reported in a way that excludes evaluation of the extent of the overall BPA effect. In contrast, Wolstenhome et al. (2011) did not find effects on anxiety in the Elevated Plus maze following oral administration of BPA.

In outbred deer mice (Peromyscus maniculatus bairdii) (Jasarevic et al. 2012), BPA at doses of $50 \mathrm{mg}$, and $5 \mathrm{mg} \mathrm{kg}$ feed weight showed increased anxiety in the Elevated Plus Maze and reduced exploratory behaviours in male offspring whose dams were fed with a diet supplemented with either ethinyl estradiol or BPA $(50 \mathrm{mg}, 5 \mathrm{mg}$, or $50 \mu \mathrm{g} / \mathrm{kg}$ feed weight) starting from 2 weeks before mating up to the end of the lactation period. The drawback of the study is that food consumption was not measured, and, therefore, the BPA doses were calculated by a formula were imprecise.

Patisaul et al. (2012) exposed Wistar rats via drinking water $(1 \mathrm{mg} / \mathrm{L})$ in the intrauterine and postnatal period (GD 6 through PND 40) to BPA (estimated dose of BPA between 100 and $1000 \mu \mathrm{g} / \mathrm{kg} \mathrm{b.w./day).} \mathrm{BPA-exposed} \mathrm{animals} \mathrm{showed} \mathrm{increased} \mathrm{anxiety} \mathrm{as} \mathrm{juveniles,}$ and as adults displayed a disappearance of the normal sexual dimorphism in exploratory behavior. Down-regulation of expression of ERß in the amygdale was reported, a key area in modulation of affective responses. Administration of a soy-enriched diet appeared to mitigate the BPA effects.

$\mathrm{Xu}$ et al. (2012) explored in mice the effects of different exposure windows (GD 7-20 or PND 1-14) with BPA (0.4 or $4 \mathrm{mg} / \mathrm{kg}$ b.w./day) by oral route. Both exposure periods and both doses increased anxiety- and depression-like behaviours in mice of both sexes measured by EPM, Open Field, dark light transition task and mirrored maze. The gestational exposure exhibited a stronger effect on anxiety-like state only in females. AMPA GluR1 receptor subunit was inhibited inhippocampus and amygdala in both sexes. 
Gioiosa et al. (2013) exposed mouse dams from GD 11 to PND 8 with an oral low-dose of BPA $(10 \mu \mathrm{g} / \mathrm{kg}$ b.w./day). After birth, offspring of exposed mothers were cross-fostered by mothers who were not exposed, whereas offspring from not-exposed mothers were nursed by exposed mothers. The exposed F1 animals were tested in EPM, open-field and novelty test to measure anxiety and emotional response to novelty. There were sex differences in the control group: control females were less anxious, more active and more prone to explore a novel environment than control males. Females exposed to BPA pre- and postnatally showed evidence of increased anxiety and were less prone to explore a novel environment relative to the control females, showing a behavioural profile more similar to control males than females. In this study, the direction of the behavioural changes was consistent and affected similarly by the pre- and postnatal exposures, although with a greater effect associated with postnatal exposure only in females. The study has limitations due to incomplete statistical considerations, as no adjustment of the $p$-value $(p<0.05)$ was made for multiple testing, although 15 endpoints were evaluated with six comparisons per endpoint. In addition, the authors investigated only one dose level of BPA. Thus, the results cannot be used for risk assessment.

In the study of Kundakovic et al. (2013), BALB/c mice were exposed from the day of mating to the end of pregnancy to BPA $(2,20$ or $200 \mu \mathrm{g} / \mathrm{kg} \mathrm{b.w./day).} \mathrm{Whole} \mathrm{arrays} \mathrm{of}$ endpoints were evaluated. Six behavioural endpoints, including anxiety-like behaviour, were assessed and expression of five genes at two locations in the brain as well as DNA methylation at eleven loci and two brain regions were measured. The results showed that maternal exposure during pregnancy induces sex-specific, dose-dependent (linear and curvilinear), and brain region- specific changes in expression of genes encoding estrogen receptors (ERs; ERa, ER $\mathrm{Er} Y$ ) and altered mRNA levels of epigenetic regulators DNA methyltransferase (DNMT) 1 and DNMT3A in the juvenile cortex and hypothalamus, paralleling changes in estrogen-related receptors. At the behavioural level, BPA exposure induced persistent, largely sex-specific effects on social and anxiety-like behaviour, leading to disruption of sexually dimorphic behaviours in adult mice. The results may only be seen as generating a hypothesis, as the statistical model is a not-prespecified exploratory model.

The study by Diaz Weinstein et al. (2013) reported an increased anxiety-like behaviour of adolescent Sprague Dawley rats (7 weeks of age) in the Open field ( $6 \mathrm{~min}$ ) test and EPM ( $5 \mathrm{~min}$ ) tests after twelve days of subcutaneous exposure to one dose level of $40 \mu \mathrm{g} / \mathrm{kg}$ bw BPA. Fujimoto et al. (2013) examined anxiety-like behaviour of Wistar rat offspring after postnatal BPA exposure through dams milk using an Open field test, an Elevated Plus Maze (EPM) and a Forced swim task at the age of six, seven and nine weeks, respectively and found that BPA did not enhance anxiety-like behaviour.

Most of the studies, with the exception of the study of Kundakovic et al.(2013) that found decreased anxiety in males, reported increased anxiety, mainly in males but occasionally also in females following in utero exposure and exposure during lactation. The doses of BPA were lower than $5 \mathrm{mg} / \mathrm{kg} \mathrm{b.w./day.}$

\section{Effects on learning and memory}

In previous studies (Xu et al., 2010; Tian et al., 2010; Carr et al., 2003), developmental exposure to BPA seemed to interfere with learning and memory capacities in different learning tasks in rodents. However, the studies were not considered valid for risk assessment due to methodological shortcomings. In addition, in the study by Stump et al. (2010, see description of tests applied and results above), the authors did not report any effects on learning and memory.

In the study by Xu et al. (2013), adult mice were exposed to oral doses of BPA $(0.4,4$, or $40 \mathrm{mg} / \mathrm{kg} \mathrm{b.w./day)} \mathrm{for} 12$ weeks. Mice were assessed at the end of treatment in two learning tasks, the Morris Water Maze and the Passive Avoidance test. BPA (0.4 or 40 $\mathrm{mg} / \mathrm{kg} /$ day) extended the average escape path length to the hidden platform in Morris Water Maze task and shortened the step-down latency $24 \mathrm{~h}$ after footshock of the males, 
but no changes were found in females. BPA reduced numeric synaptic density and an enlarged synaptic cleft and reduced length of active zone and PSD thickness, in the hippocampus of male mice. Western blot analyses further indicated that BPA downregulated expressions of synaptic proteins (synapsin I and PSD-95) and synaptic NMDA receptor subunit NR1 and AMPA receptor subunit GluR1 in the hippocampus of the males.

Eilam-Stock et al., 2012 investigated the effects of a single subcutaneous BPA injection $(40 \mu \mathrm{g} / \mathrm{kg}$ b.w.) on memory and synaptic plasticity in adult male rats. Memory tests applied included the Object Recognition (OR) and the Object Placement (OP) tasks. The authors reported that BPA significantly impaired both OR and OP and decreased spine density in the hippocampus and medial prefrontal cortex. Additionally, BPA significantly decreased PSD-95, a measure of neural plasticity in the hippocampus and increased PCREB, a transcription factor, in the prefrontal cortex. Together, these findings show that BPA may block the formation of new memories by interfering with neural plasticity processes in the adult brain. The Panel noted that the study was performed in adult animals, limiting its value in assessment of developmental neurotoxicity.

In contrast to the results by Diaz Weinstein et al. (2013) and Eilam-Stock et al. (2012) the study by Inagaki and coworkers (2012), performed in adult ovariectomised (OVX) female 0 rats (to abolish any estrogenic modulation of behaviour) dosed by acute subcutaneous injectionswith BPA at levels from $0.4 \mu \mathrm{g} / \mathrm{kg} \mathrm{b} . w$. to $400 \mu \mathrm{g} / \mathrm{kg} \mathrm{b} . \mathrm{w}$. (6 does levels), found that BPA did not impair memory response per se in either OR and OP memory task, but it significantly antagonized the effects of $17 \beta$ estradiol as enhancer of learning and memory performances and dendritic spine formation (lowest dose of BPA effective in blocking the facilitatory effect of estradiol in OP test equal to $4 \mu \mathrm{g} / \mathrm{kg} \mathrm{b.w}$.; in OR test equal to $40 \mu \mathrm{g} / \mathrm{kg} \mathrm{b.w.).} \mathrm{A} \mathrm{group} \mathrm{of} \mathrm{normally} \mathrm{cycling} \mathrm{rats} \mathrm{were} \mathrm{also} \mathrm{used} \mathrm{and}$ exposed to a single dose level of $40 \mu \mathrm{g} / \mathrm{kg}$ b.w.: while BPA did not affect OP performance at any phase of the estrous cycle, OR memory was inhibited by BPA only on proestrous when endogenous E2 levels are at the highest.

These two studies, though exploring the effects of a single administration of BPA in adult animals, added some mechanistic information to explain the effects reported for developmental BPA on learning and memory processes and spinogenesis, which could implicate the interference of BPA with steroid-modulated synaptogenesis occurring during brain ontogenesis in physiological conditions (see the review by Hajszan and Leranth, 2010).

\section{Studies with exposure during pregnancy}

Jones and Watson (2012) failed to evidence any effects of oral gavage of BPA (doses 5, 50,500 , and $5000 \mu \mathrm{g} / \mathrm{kg}$ b.w./day) during gestation and lactation on spatial learning in the Morris Water Maze.

On the contrary, Jasarevic et al. (2012) reported that male deer mice orally exposed during gestation and lactation to $0.05,5$ or $50 \mathrm{mg} / \mathrm{kg}$ feed weight BPA equivalent to $0.25,25$ or $250 \mu \mathrm{g} / \mathrm{kg}$ b.w./day had impaired learning performance in the Barnes Maze, while females outperformed males.

Finally, Ferguson et al. (2012) in their robust study using two very low doses of BPA (2.5 or $25 \mu \mathrm{g} / \mathrm{kg}$ b.w./day) given by oral gavage on gestational days $6-21$ and then to offspring from birth to weaning did not show significant effects on spatial learning.

There are several papers presenting evidence indicating effects of BPA exposure during development on social responses, including social/affiliative interactions in female rodents, sexual behaviour and aggression in males ( $\mathrm{Xu}$ et al., 2011; Jones et al., 2011; Cox et al., 2010; Tian et al., 2010; Palanza et al., 2008; Patisaul and Bateman, 2008; Giosa et al., 2007). Overall, the direction of the effects ranged from pro-social effects to reduction of social motivation. Although most of these studies have been reviewed by EFSA in 2010, the social behaviour endpoint was not addressed separately in the EFSA 2010 opinion. 
In the 2011 study by Wolstenholme et al., the female offspring born to females fed with a BPA-supplemented diet during pregnancy (about $1.25 \mathrm{mg} \mathrm{BPA} / \mathrm{kg}$ diet estimated to be equivalent to approximately $120 \mu \mathrm{g} / \mathrm{kg}$ b.w./day) showed slightly increased social interactions in a free 30-min social interaction test. The effect on males was in the same direction, but less significant. However, BPA did not affect social preference for a stimulus animal when compared to an inanimate object. Gene expression analysis performed in whole-brain embryos revealed mRNA for the glutamate transporter Slc1a1 was enhanced by exposure to BPA in female brains and that expression of two of the three DNA methyltransferase genes, Dnmt1 and Dnmt3a, was modulated by BPA. Notably, expression of estrogen receptor genes was not affected by BPA, but oxytocin receptor gene (highly responsive to estrogen modulation and involved in social behaviour) was reduced in males.

In a second study assessing transgenerational effects of BPA in mice through four generations, Wolstenholme et al. (2012) exposed the F0 generation only to BPA (about 5 $\mathrm{mg} / \mathrm{kg}$ diet, equivalent to approximately $1.0 \mathrm{mg} / \mathrm{kg} \mathrm{b.w./day21)} \mathrm{through} \mathrm{pregnancy} \mathrm{and}$ lactation. Subsequent generations were not exposed to BPA. Brains from embryos from mothers exposed to BPA had lower gene transcript levels for several estrogen receptors, oxytocin, and vasopressin as compared with controls in the F1 generation; decreased vasopressin mRNA persisted into the F4 generation, at which time oxytocin was also reduced but only in males. Changes in gene expression were paralleled by alterations in social behaviour in F1 and in F2 and F4. The effects were in the direction of slightly reduced social interaction in F1 and increased social interaction in either F2 and F4.

The CEF panel noted that these two studies have some methodological limitations (only one dose level used, litter effect not properly controlled). However, the extent of the effects on $\mathrm{F} 1$ in the two studies is comparable although possibly in two opposite directions (considering that different BPA dosages were used). The different direction of effects in F2 and F4 was an unexpected result and might suggest inconsistency, but transgenerational effects might result from modulation of several genes implicated in the control of complex functional end points.

\section{Effects on sensory-motor functions}

In previous studies, no convincing evidence of a consistent BPA-related effect on motor activity was demonstrated at low oral doses (Stump, 2010).

Newer studies on changes in sensory-motor function following pre- and post-natal exposure were published by Ferguson et al. (2012) and Viberg et al. (2011).

Ferguson et al., 2012 studied in rats the effect of exposure towards 2.5 and $25 \mu \mathrm{g} / \mathrm{kg}$ b.w. daily from GD 6 to 21 and PND 1 to 21 . Whereas in positive controls (5 and 10 $\mu \mathrm{g} / \mathrm{kg}$ b.w./day EE), clear effects were seen in open field assessments and the Barnes maze escape box, few consistent or dose-related effects resulted from developmental treatment with BPA at the doses tested.

Viberg et al. (2011) showed that there were significant alterations in behavior and cognitive functions in mice after two months and after five months, for the locomotion, rearing, and total activity variables after a single oral administration of 3.3 or $4.8 \mathrm{mg} / \mathrm{kg}$ b.w. BPA but not of $0.23 \mathrm{mg} / \mathrm{kg}$ b.w. on PND 10 .

Studies with endpoints in brain biochemistry, neurogenesis, neuroanatomy and gene expression (ex vivo studies)

The study by Cao et al. (2012) shows that $50 \mu \mathrm{g} / \mathrm{kg}$ b.w. BPA by subcutaneous injection daily from postnatal day 0 (PND 0) to PND 2 had regional and sex-specific alterations of gene expression of estrogen receptor alpha (ERa), ER beta (ER $\beta$ ) and kisspeptin (Kiss1) that are all decreased in the anterior and mediobasal hypothalamus on PND 4 and 10. Notably, the effects of BPA are very different from those of estradiol (positive control), supporting the view that the interference of BPA with early hypothalamic organization involves mechanisms different from its estrogenic action. A more recent study by Cao et al. (2013) found that offspring of rats receiving BPA orally from gestational day 6 to PND 
21 (2.5 or $25 \mu \mathrm{g} / \mathrm{kg}$ b.w.) show significant changes in estrogen receptors ESR1 and ESR2 in hypothalamus and amygdale at birth. Specifically, both doses of BPA increased expression of ESRs in both sexes comparably to 5 or $10 \mu \mathrm{g} / \mathrm{kg} \mathrm{b} . w$. ethinyl estradiol.

The study by He, Paule and Ferguson (2012) indicates that BPA can have sex-specific effects on hypothalamic medial preoptic area volume and that these effects manifest as larger volumes in males, with oral exposure during pregnancy and by gavage to the pups during the period of lactation at doses of 2.5 or $25 \mu \mathrm{g} / \mathrm{kg} \mathrm{b}$.w. These alterations in rats have been linked to changes in sexual dimorphic behaviour. The consequences of these morphological changes if replicated in humans are not known, but the medial preoptic area has a pivotal role in the regulation of sexual and parental behaviour in mammals including primates.

Two studies addressed the controversial issue of BPA effects on hippocampal neurogenesis (considered as a clear adverse effect by the 2013 ANSES report). Kim et al. (2011) found increased neurogenesis after treating mice by oral gavage for 2 weeks in late adolescence with $1 \mathrm{mg} / \mathrm{kg}$ b.w./day oral BPA, whereas Komada et al.(2012) described a similar effect in the fetus after in utero exposure to $200 \mu \mathrm{g} / \mathrm{kg} \mathrm{b} . \mathrm{w} . /$ day.

In the study by Xu et al. (2013), mouse dams were orally exposed to BPA (4, 0.4 or 0.04 $\mathrm{mg} / \mathrm{kg}$ b.w./day) from GD 7 through PND 21. Results showed that BPA (lower and higher doses) significantly reduced the numeric synaptic density of pyramidal cells in hippocampus CA1 region on PND 14, 21 and 54 in maleoffspring ( $p<0.001$ ). The reduced density was paralleled by significant modification of structural parameters indicative of synaptic functionality (enlargement of synaptic cleft by 0.4 and $4 \mathrm{mg} / \mathrm{kg}$ BPA and reduction of the active synapse zone as indicated by decreased Post Synaptic Density at 0.4 and $4 \mathrm{mg} / \mathrm{kg}$ BPA on PND 14 and PND 21, respectively. BPA also reduced the expression of synapsyn 1 and PSD95 in a dose-dependent fashion at all the time endpoints analysed. In addition, exposure to BPA markedly reduced the expression of both glutamate NMDA and AMPA receptors in the hippocampus on PND 14, 21 and 56 at the doses of 0.04 and $4 \mathrm{mg} / \mathrm{kg} 3118 \mathrm{~b} . \mathrm{w} . /$ day.

Finally, two in vitro studies showed that BPA concentrations in the nanomolar range inhibits NGF-induced neurite extension in PC12 cells (Seki et al., 2011), while BPA significantly enhanced spinogenesis when added to isolated hippocampal slices obtained from untreated adult male Wistar rats (BPA concentrations ranging from $1 \mathrm{nM}$ to $10 \mu \mathrm{M}$ ), with mechanisms likely independent from estrogen receptors (Tanabe et al., 2012).

\section{Conclusion}

There is uncertainty with regard to the interpretation of the data regarding neurological effects of BPA. In several studies an increased anxiety was observed. However, studies on anxiety (rodent and non-human primate) have a behavioural end-point that is sensitive for a number of reasons such as study design, testing apparatus, inclusion of only one sex, and age at examination. New data confirm previous data that BPA has an effect on sex-dimorphic social behaviour. However, it is disputed whether elimination of sexual dimorphism could be considered as an adverse effect in extrapolating to humans. Gene expression in the brain was also altered either after prenatal BPA exposure and BPA exposure in adult mice. Other effects of BPA on hypothalamic organization involve mechanisms different from its estrogenic properties because the effects of oestradiol were different. However, in the studies methodological shortcomings have been observed (insufficient sample size, litter effect was not taken into consideration, uncontrolled variability of dietary exposure, flawed statistics). In addition, the different studies presented inconsistent results that do not allow firm conclusions to be drawn. However, EFSA did take into consideration possible effects on the nervous system and behaviour when performing the uncertainty analysis (EFSA 2015). 


\subsubsection{I mmunotoxicity}

In previous reviews on BPA, it was concluded that BPA is capable of inducing skin sensitization responses in humans with low prevalence being a weak sensitizer (EFSA 2010, FAO/WHO 2011, EC 2010a, ANSES 2011). Some individual cases were also reported describing contact dermatitis against BPA (Aalto-Korte et al., 2003). The results of rodent studies suggest that BPA may modulate immune homeostasis especially regarding the induction of T-cells and cytokine production directing the immune response into an allergy prone profile (EFSA 2010, FAO/WHO 2011, EC 2010a, ANSES 2011). However, the results were insufficient to conclude on immunotoxic activity of BPA, and the immune system was considered to be an area of interest for further research.

The study of Lee et al. (2012) evaluated possible mechanisms of sensitization induced by BPA through investigating the cytokine profiles after BPA exposure. Mice were injected intraperitoneally with BPA (5 mg/kg b.w.). Total non-specific IgE antibodies and $\beta$ hexosaminidase and histamine (both inducing degranulation of mast cells) in serum were increased. The results are indicative for an effect of BPA on the immune system at doses higher than the PoD considering the route of exposure, however, no specific immune responses were measured.

Kendziorsky et al. (2012) investigated the response of BPA in a specific mouse strain prone to develop pyometra. BPA was observed to induce pyometra in one of two mice strains investigated similar to $17 a-e t h i n y l$ estradiol. For both compounds, only one dose induced pyometra, and no dose-response relationship was established. The authors concluded that there was a strain specific estrogen sensitivity resulting in pyometra in $\mathrm{C} 57 \mathrm{BI} / 6$ versus CD1 mice. However, only in one out of five animals treated with BPA pyometra was observed. No effects of BPA were observed on fertility in both C57BI/ 6 and CD1 mouse strains. The results of the study are of no value for the risk assessment.

Nakajima et al. (2012) exposed mice to $10 \mu \mathrm{g} / \mathrm{ml}$ BPA in their drinking water from 1 week before pregnancy until PND 22. The treatment with BPA, followed by postnatal allergic sensitization with ovalbumin and a challenge at PND 22, promoted the development of ovalbumin-induced allergic asthmatic responses (airway hyperreactivity, increase in eosinophilic granulocytes).

BPA was given by gavage $(0,0.5,50$, or $500 \mu \mathrm{gPA} / \mathrm{kg} /$ day $)$ to pregnant $\mathrm{C} 57 \mathrm{BI} / 6$ damns (GD 6 until PND 21). Adult offspring were sensitized by mucosal route of administration or by the interaperitoneal route (Bauer et al., 2012). Mucosal sensitization was with inhaled ovalbumin containing low dose lipopolysaccharide. Intraperitoneal sensitization was performed using ovalbumin with "alum" (no details given by the authors), followed by ovalbumin aerosol challenge. Enhanced lymphocytic and lung inflammationwas observed in female animals compared to contro offspring after mucosal sensitization but not after intraperitoneal sensitization. The authors stated that the maternal exposure to BPA resulted in subtle and qualitative different effects on the allergic inflammation. However, these subtle yet persistent changes did not lead to significant differences in overall airway responsiveness, suggesting that the early life exposure to BPA did not exarcerbate allergic inflammation into adulthood. The same research group reported in a similar experimental design (BPA exposure of the mothers from GD6 to PND21) that the developmental exposure to BPA did not compromise the disease-specific adaptive immunity against an influenza virus infection, although the innate immunity was affected as indicated by a reduced pulmonary inflammation and cytokine exression (Roy et al., 2012).

In three studies, data of the US NHANES were used for evaluation of possible BPA effects on allergies (Clayton et al., 2011, Savage et al., 2012, Vaydia et al., 2012). All three studies used spot urinary BPA levels for their evaluations. Clayton et al. (2011) evaluated urinary BPA levels and the presence of viral antibodies and allergy in children older than 6 years of age. BPA showed no association with allergy diagnosis, whereas the evaluations of the CMV antibodies showed contradicting results in diferent age groups. 
Both low and high BPA urinary levels were associated with a higher antibody levels. Savage et al. (2012) evaluated urinary BPA levels and the sensitization against aeroallergens and food allergens by measurement of antigen specific IgE levels in sera. For BPA, no associations with IgE levels were observed, whereas for some other chemicals investigated an association was observed. However, Vaydia et al. (2012) did find an association between urinary BPA levels and allergic asthma based on total IgE determinations and allergen specific IgE levels. BPA was associated with a higher likelihood of allergic asthma in females but not in males.

In two studies, BPA exposure of mothers and presence of wheeze in their children was investigated. Spanier et al. (2012) measured spot urinary levels in pregnant women at week 16 and 26 of gestation and at birth. In general, BPA levels were not associated with the occurrence of wheeze in the children. However, mean prenatal BPA above versus below the median was positively associated with wheeze at 6 months of age but not at 3 years. Another mother and child cohort was reported by Donohue et al. (2013), in which urinary BPA levels were evaluated in relation to occurrence of wheeze. Higher prenatal BPA levels were associated with a lower ocurrence of wheeze at five years of age. In contrast, post-natal BPA levels in the children indicated that higher BPA levels were associated with an increase in wheeze and asthma.

\section{Conclusion}

BPA is able to elicit skin sensitization in humans, probably as a result of it being a weak sensitizer. Studies on a possible relationship between prenatal and/or postnatal BPA exposure and allergic responses are not consistent. Other studies on immunotoxic responses are insufficient to draw final conclusions: although effects on the immune system are suggested, there is uncertainty on the immunotoxicity of BPA. However, EFSA did take into consideration possible effects on the immune system when performing the uncertainty analysis (EFSA, 2015).

\subsubsection{Cardiovascular effects}

None of the large-scale experimental animal studies (90 days, 2 years carcinogenicity study) suggest effects on cardiovascular function (see Risk Assessment Report ECECB $2003,2008)$. In conclusion, the toxicological data do not indicate a clear effect of BPA on cardiovascular function.

\subsubsection{Metabolic disorders}

\section{Summary of previous opinions}

In the EU-RAR of 2003, updated in 2010 (ECB 2003, EC 2010a,b), metabolic effects of BPA were not mentioned. Whereas EFSA did not give reports addressing the effects of BPA on the metabolism of experimental animals in the 2006 Opinion (EFSA 2006), in the 2010 EFSA Opinion (EFSA 2010) publications were cited with effects of BPA on insulin secretion in mice (Ropero et al., 2008) and increased adipogenesis in the female offspring of rats exposed prenatally to BPA (mean oral dose $70 \mu \mathrm{g} / \mathrm{kg} \mathrm{b.w./day)} \mathrm{(Somm}$ et al., 2009) and aggravated insulin resistance in mice during pregnancy at s.c. doses of 10 or $100 \mu \mathrm{g} / \mathrm{kg} / \mathrm{d}$ (Alonso-Magdalena et al., 2010). The study of Ryan et al. (2010) was cited showing no indications of increased susceptibility to induced obesity by high fat diet and of glucose intolerance in adult mice exposed prenatally to BPA $(0.25 \mu \mathrm{g} / \mathrm{kg} \mathrm{b} . \mathrm{w} . / \mathrm{day}$ orally). The NTP-CEHR monograph (US NTP 2008) reviewed the study of AlonsoMagdalena et al. (2006) and the study of Miyakawa et al. (2007). Because of the limited data, the NTP-CEHR monograph did not make a firm conclusion concerning metabolic effects of BPA. In the FAO-WHO opinion (2011) the experts expressed their view that the 
data of Miyakawa et al. (2007), Somm et al. (2009), Alonso-Magdalena et al. (2010) and Ryan et al. (2010) warranted further assessment of the potential effects of BPA on adiposity, glucose or insulin regulation, lipids and other end-points related to diabetes or metabolic syndrome.

The 2011 ANSES report reviewed the studies also considered by FAO-WHO as well as a study by Rubin et al. (2001). This study showed obesity in the offspring of SpragueDawley female exposed via drinking water, at approximately $0.1 \mathrm{mg}$ or $1.2 \mathrm{mg}$ from GD6 throughout the period of lactation. According to the ANSES report, effects of BPA on lipogenesis in experimental animals were proven (including adipocyte hypertrophy, predisposition to obesity, elevated cholesterol levels and triglyceride levels and overexpression of lipogenic proteins) following pre- and perinatal exposure in adults. The ANSES risk assessment report (2013) confirmed this view and stated that the increase in body weight in experimental animal studies, together with increases in plasma lipids (such as cholesterol and triglycerides) and lipogenesis, are critical effects. According to ANSES (2013), the Miyakawa et al. (2007) study in ICR mice is considered the pivotal study for risk assessment, and a LOAEL of $0.26 \mathrm{mg} / \mathrm{kg} \mathrm{b.w./day} \mathrm{was} \mathrm{derived} \mathrm{based} \mathrm{on} \mathrm{an}$ increase in body weight and an increase in cholesterolemia in females.

Since the EFSA opinion of 2010, the WHO Expert meeting of 2010 and the ANSES report of 2011, several additional experimental studies have reported metabolic effects of BPA (including effects on body weight/obesity, lipogenesis or adipogenesis) and/or effects related to glucose or insulin regulation. Studies published over the last 5 years include Miyawaki et al., 2007; Somm et al., 2009; Alonso-Magdalena et al., 2010; Ryan et al., 2010 and Wei et al., 2011. Findings from these studies include reports of glucose intolerance and hyperinsulinaemia in the 6-month-old male offspring of OF-1 mice treated from GD9 to GD16 with BPA at 10 or $100 \mu \mathrm{g} / \mathrm{kg}$ b.w. per day. In the study by Alonso-Magdalena et al. (2010), the administration was by subcutaneous injection of 10 $\mu \mathrm{g} / \mathrm{kg} \mathrm{b.w./day} \mathrm{or} 100 \mu \mathrm{g} / \mathrm{kg}$ b.w./day. Importantly, s.c. administration has a systemic availability of $100 \%$, whereas the systemic availability of an oral dose is $2 \%$ (Doerge et al., 2012). Thus, $10 \mu \mathrm{g} / \mathrm{kg}$ b.w./ day and $100 \mu \mathrm{g} / \mathrm{kg}$ b.w./day s.c. corresponds to 500 $\mu \mathrm{g} / \mathrm{kgb} . \mathrm{w} . / \mathrm{day}$ and $5000 \mu \mathrm{g} / \mathrm{kg}$ b.w./day by the oral route. The endpoints measured were manifold and the dose-response relationship was not monotonic for all of them. Somm et al. (2009) observed adipocyte hypertrophy and increased mass of parametrial white adipose and brown adipose tissue on postnatal day (PND) 21 in female offspring of Sprague-Dawley rats who were orally treated with BPA approximately $70 \mu \mathrm{g} / \mathrm{kg} \mathrm{b.w}$. per day in drinking-water from GD 6 to PND 21. Furthermore, increased cholesterol on PND 31 was observed in female offspring of ICR mice orally treated with BPA (in drinkingwater) at approximately 260 or $2600 \mu \mathrm{g} / \mathrm{kg}$ b.w. per day. Exposure was from GD 10 to weaning via the dam and then after weaning with the same drinking water treatment as the dam (Miyawaki et al., 2007). In the most recent study of Wei at al. (2011), doses of $50 \mu \mathrm{g} / \mathrm{kg}, 250 \mu \mathrm{g} / \mathrm{kg}$ and $1250 \mu \mathrm{g} / \mathrm{kg}$ were given by oral gavage throughout gestation and lactation.

Effects were increased body weight, elevated serum insulin and impaired glucose tolerance in adult offspring. It was striking that effects were only observed at the 50 $\mu \mathrm{g} / \mathrm{kg} / \mathrm{d}$ dose and only in male offspring. Effects in males were accelerated and more severe when offspring were fed a high-fat diet. In this group, severe metabolic syndrome, dyslipidaemia, hyperleptinaemia, hyperglycaemia, hyerinsulinaemia and glucose intolerance were observed. In contrast, Ryan et al. (2010) observed changes in body weight and size in mice that are no longer apparent when the animals reach adulthood. Taken together, the study results are inconsistent: the effects were seen only in females (Somm et al., 2009), but also only in males (Wei et al., 2011). Additionally, doses where effects were observed are at variance in the studies and contradictory results have been observed.

Animal studies have shown an increase (Miyawaki et al., 2007; Rubin et al., 2001; Somm et al., 2009, Wei et al., 2011, Mackay et al., 2013), a decrease (Honma et al., 2002; 
Nagel et al., 1997), or no effect on body weight (Ryan et al., 2010, Marmugi et al., 2012, Mackay et al., 2013, Anderson et al., 2013) after early life exposure to BPA and the effect can occur in both or only one sex (Somm et al., 2009; Alonso-Magdalena et al., 2010, Mackay et al., 2013). The discrepancy among these animal studies may arise from variety of experimental conditions, such as dosing regimens, animal species and strains, and timing of evaluation of effects.

\section{$\underline{\text { In vivo studies studies involving prenatal exposure }}$}

Xu et al. (2011) suggested that an increased preference of adult rats for a sweet taste, potentially resulting in obesity, could be linked to prenatal exposure to BPA. Female Sprague Dawley rats were exposed to BPA in drinking water at doses of $0.01,0.1$ and $1.0 \mathrm{mg} / \mathrm{L}$ from G11 to lactation day 21. A significant sex difference in preference for a sweet taste was evident in both BPA-treated and non-BPA-treated offspring, with all females including controls showing a preference for saccharin-containing drinking water compared with plain water. There was no evidence of a treatment-related effect. However, male offspring showed an increased preference for $0.25 \%$ (but not for $0.5 \%$ ) saccharin, and for $15 \%$ sucrose, compared with male controls. The preference for $15 \%$ sucrose was reversed in BPA-treated females compared with controls, implying the feminization of males and masculinization of females. Male offspring from dams receiving $0.1 \mathrm{mg} / \mathrm{L}$ BPA who were administered $15 \%$ sucrose in their drinking water postnatally also showed increased body weight gain, a higher percentage of body fat and higher tail blood pressure compared to the control group. The inconsistency in the response to saccharin (preference for $0.25 \%$ but not for $0.5 \%$ saccharin) is noted, interpretation of the saccharin preference results was difficult. In addition, there is no explanation why only the middle dose of BPA pups for the sucrose preference test was chosen. These drawbacks limit the conclusions that can be drawn from the study.

Wei et al. (2011) administered doses of $0,50,250$ or $1250 \mu$ BPA $/ \mathrm{kg} \mathrm{b.w.} \mathrm{per} \mathrm{day} \mathrm{orally}$ by gavage in corn oil to pregnant Wistar rats from GD0 to PND2. The offspring ( $n=16$ per group, 2 from each of 8 litters) were maintained on either a normal or a high fat diet for 16 weeks, with monitoring of body weight and blood parameters (triglycerides, cholesterol, low- and high-density lipoprotein) and periodic glucose tolerance and insulin tolerance tests throughout the experimental period: morphology and function of the pancreas was assessed at termination at week 27 . The authors present only results for the $50 \mu \mathrm{g} / \mathrm{kg}$ b.w./day dose in their paper, which limits the interpretation of the data. Offspring exposed prenatally to $50 \mu \mathrm{g} \mathrm{BPA} / \mathrm{kg}$ b.w. per day and maintained on a normal diet showed increased weight gain from week 17 (females) or week 19 (males), and serum insulin levels were higher at week 15 for males and at week 26 for females. Effects were more evident in animals fed a high fat diet. No effects of BPA were observed at the two higher doses (250 or $1250 \mu \mathrm{g} \mathrm{BPA} / \mathrm{kg}$ b.w. per day). Serum leptin was elevated in 50 ug BPA $/ \mathrm{kg}$ b.w. BPA-treated animals compared with controls at week 26; the animals also had a higher body fat percentage and showed hypertrophy of adipocytes. Mitochondrial structure and insulin granule characteristics in pancreatic $\beta$ cells were altered by BPA at $50 \mathrm{ug} / \mathrm{kg}$ b.w. per day and mRNA expression of isletassociated transcription factors were reduced compared to controls. This investigation was carried out in the $50 \mu \mathrm{g} / \mathrm{kg}$ b.w./day group only.

In the study of Mackay et al. (2013), CD mice were exposed from GD 1 until PND21 to diets containing 0,1 or $20 \mu \mathrm{g} \mathrm{BPA} / \mathrm{kg}$, estimated to be equivalent to an average of 0.19 and $3.49 \mu \mathrm{g} / \mathrm{kg}$ b.w. per day prenatally and 0.36 and $7.2 \mu \mathrm{g} / \mathrm{kg}$ b.w. per day of BPA postnatally. Offspring were weaned initially onto a normal diet, then as adults exposed to either a normal or high-fat diet (HFD). Female offspring at the higher BPA dose level and fed a high fat diet showed increased body weight gain as adults compared with controls and the DES positive control, and also ate more. They had increased adiposity and leptin concentrations with reduced propio-melanocortin mRNA expression in the arcuate nucleus and estrogen receptor a expression patterns similar to those seen in males, 
which the authors considered to be suggestive of a masculinising effect of BPA. Male offspring showed no similar BPA-linked effect on body weight gain; however, males at both levels of BPA showed a dose-related increase in weight in the retroperitoneal and intrascapular brown adipose fat pads compared with control and DES-exposed mice, and similar effects were seen in female offspring at the higher dose but not at the lower level of BPA. The extent of the effects was small. Males exposed to the high dose of BPA showed impaired glucose tolerance on both diets.

Anderson et al. (2013) exposed mice starting at two weeks before mating until the end of lactation (PND 21) to $0,50 \mathrm{ng}, 50 \mu \mathrm{g}$ or $50 \mathrm{mg}$ of BPA/ $\mathrm{kg}$ of diet corresponding to 0 , $10.75 \mathrm{ng}, 10.75 \mu \mathrm{g}$, and $10.75 \mathrm{mg} / \mathrm{kg}$ b.w./day. A subset of animals, 1 male and 1 female/litter, was followed until 10 months of age on standard diet or diets containing BPA at the same levels as administered to the dams. The authors found increased energy expenditure as evidenced by increased oxygen consumption and carbon dioxide production in all BPA-treated animals. Notably, however, the dose-response relationship was inconsistent. Spontaneous activity was increased, but only in females. Food consumption in females was reduced to a statistically significant extent but without a clear dose-response, whereas in males the reduction of food intake was not statistically significant. Body weight and body fat was not statistically different from control in either sex and glucose tolerance and insulin release were also unchanged.

A further study was published by Angle et al. (2013). Pregnant CD-1 mice BPA were given BPA at oral doses ranging from $5-50,000 \mu \mathrm{g} / \mathrm{kg} /$ day from GD 8 until GD 18 . In the male offspring from the animals, a large number of endpoints were measured (body weight; gonadal and renal fat pad weight; adipocyte number and adipocyte volume; food (metabolic energy) consumption; glucose and insulin tolerance tests; serum hormones (such as insulin, leptin, adiponectin) at different time points and statistical assessment explored several models. Most of the endpoints showed non-monotonic changes which are difficult to assess. No pathophysiolocal model can be derived from the data and no biological explanation can be given at present.

In the study of US FDA/NCTR (Delclos et al., 2014) in Sprague-Dawley rats, no effect of BPA on metabolic parameters was observed. Doses were $2.5,8,25,80,260,840,2,700$, 100,000 , and $300,000 \mu \mathrm{g} / \mathrm{kg}$ bw per day given by direct gavaging from GD 6 until PND 90.

$\underline{\text { In vivo studies in adult mice and rats }}$

D'Cruz et al. (2012) dosed male rats ( $n=6$ per group) with BPA $(0.005,0.5,50$ and 500 $\mu \mathrm{g} / \mathrm{kg}$ b.w. per day orally) for 45 days. $17-\beta$-estradiol $(50 \mu \mathrm{g} / \mathrm{kg}$ b.w./day) was used as a positive control. A whole array of endpoints was measured: Plasma glucose, plasma insulin and enzymes involved in glucose metabolism were investigated. In addition, testicular levels of insulin, insulin signalling molecules, glucose transporter-2, antioxidant enzymes and steroidogenesis were also evaluated. Levels of plasma glucose and insulin were significantly increased even at the lowest level of BPA exposure of $5 \mathrm{ng} / \mathrm{kg} \mathrm{b.w}$. per day, whereas the testicular glucose level significantly decreased, again at all dose levels. Levels of insulin and various insulin signalling molecules were significantly decreased in testis by BPA in a dose-related manner even at the lowest dose of $5 \mathrm{ng} / \mathrm{kg} \mathrm{b} . \mathrm{w} . /$ day. A significant decrease in testicular superoxide dismutase and catalase activities was measured following BPA exposure at all doses, and lipid peroxidation was increased, together with decreases in testicular marker proteins and key enzymes of steroidogenesis. There was evidence of testicular damage as evidenced by loss of germ cells and decrease in the spermatids in rats treated with $500 \mu \mathrm{g} \mathrm{BPA}$, as well as in the positive control. The authors concluded that low doses of BPA affect insulin signalling and glucose, possibly leading to impairment of testicular function.

Batista et al. (2012) administered subcutaneously a dose of $100 \mu \mathrm{g}$ BPA/kg b.w./day divided into two subcutaneous injections to 3-month old Swiss albino OF1 mice for 8 days. Whole body energy homeostasis and responses of insulin sensitive peripheral tissues were assessed. Higher plasma insulin concentrations in the fed state and 
increased glucose-stimulated insulin secretion in isolated pancreatic islet of Langerhans were shown for BPA treated animals, in addition to changes in insulin signaling. Glucose tolerance testing showed that BPA-treated mice were insulin resistant. Whole-body energy homeostasis, as assessed by food intake, locomotor behaviour and nocturnal energy expenditure was reduced. In contrast, the respiratory exchange ratio was unchanged. Hence, the findings of this study were contradictory in themselves.

Male CD1 mice were dosed with $0,0.05,0.5,5$ or $50 \mathrm{mg} / \mathrm{kg}$ BPA in the diet, estimated by the authors to be equivalent to $0,5,50,500$ and $5000 \mu \mathrm{g} / \mathrm{kg} \mathrm{b.w./day} \mathrm{for} 28$ days (Marmugi et al., 2012). After the treatment period, measurements were taken for body weight gain, liver weight and weight of perigonadic white adipose tissue (pWAT), hepatic lipid content and fatty acid composition, plasma levels of insulin, triglycerides, glucose, total cholesterol, and low- or high-density lipoprotein (LDL, HDL) cholesterol. In addition, the effects of BPA on gene expression in the liver were assessed using microarrays. No effect was seen on body weight gain and relative liver weight, but pWAT weight was significantly increased in mice receiving $50 \mu \mathrm{g} / \mathrm{kg}$ b.w./day (but not at higher dose levels). Plasma insulin levels were significantly increased following exposure to 5, 50, and $500 \mu \mathrm{g} \mathrm{BPA} / \mathrm{kg}$ b.w./day: the lowest dose produced the greatest effect. Plasma glucose and tota, LDL- or HDL-cholesterol were not different from control. Mice exposed to 500 $\mu \mathrm{g}$ BPA $/ \mathrm{kg}$ b.w./day showed a significant increase in plasma triglyceride levels. The effects were confirmed in a further study in C57BL/6] mice given the same BPA doses, although the changes were generally of a smaller magnitude. The results of the microarray assays showed a stimulatory effect of BPA on expression of key enzymes involved in lipogenesis, cholesterol biosynthesis and, to a lesser extent, enzymes involved in glucose metabolism. A stronger response was seen in the liver of mice dosed with $50 \mu \mathrm{g} / \mathrm{kg}$ b.w./ day than those dosed with $5000 \mu \mathrm{g} / \mathrm{kg}$ b.w./day. Hence, a nonmonotonic dose-response seems to exist.

Bodin et al. (2013) investigated possible effects of BPA on the development of type 1 diabetes (T1DM). They gave 0,1 and $100 \mathrm{mg} / \mathrm{L} \mathrm{BPA}$ in the drinking water (corresponding to intakes of 0,150 or $15,000 \mu \mathrm{g} / \mathrm{kg} \mathrm{b.w./day)} \mathrm{to} \mathrm{non-obese} \mathrm{pre-diabetic} \mathrm{(NOD)} \mathrm{mice.}$ The prevalence and extent of insulitis did not differ between groups at week 7. At week 12 , markedly increased effects were seen compared with controls only in female mice and only exposed to $1 \mathrm{mg} / \mathrm{l} \mathrm{BPA}$ in drinking water, being less severe in the dosing group of $100 \mathrm{mg} / \mathrm{l}$. Prevalence and extent of insulitis was decreased in male mice exposed to BPA compared with controls. Serum glucose levels were increased in the $1 \mathrm{mg} / \mathrm{ml}$ BPA group, indicating an accelerated onset of T1DM, but this was not seen in the animals exposed to $100 \mathrm{mg} / \mathrm{l}$ BPA. Insulin levels did not differ significantly between the groups. Serum levels of T4, cytokines and autoantibodies did not differ between the groups.

In the studies of the Jayashree group (Jayashree et al., 2013; Indumathi et al., 2013), BPA $(0,20$ or $200 \mathrm{mg} / \mathrm{kg}$ b.w./day orally) was administered for 30 days to adult male Wistar rats. The effects of BPA were investigated on insulin signal transduction and glucose oxidation in skeletal muscle and liver. After 30 days of treatment serum insulin was significantly increased by BPA in a dose-related manner, whereas glucose oxidation was reduced at both dose levels in liver and in skeletal muscle, and glycogen content of the liver was also reduced. In skeletal muscle, treatment with BPA at both 20 and 200 $\mathrm{mg} / \mathrm{kg}$ b.w., significantly decreased the levels of insulin receptor, of protein kinase $B$ and of glucose transporter-4 levels (both plasma membrane and cytosolic fraction). However, the mRNA levels for these proteins were unchanged. In the liver, both mRNA and protein levels were significantly decreased at the highest BPA-exposed group.

Female F-344 rats were dosed with $0.025,0.25$ or $2.5 \mathrm{mg} \mathrm{BPA} / \mathrm{L}$ in drinking water from five to 15 weeks of age (Rönn et al., 2013). The drinking water contained $5 \%$ fructose ( $n=12$ per group) and BPA intake, calculated by the authors, was between 4.6 (week 9) and 5.6 (week 2) $\mu \mathrm{g} / \mathrm{kg} \mathrm{b.w./day} \mathrm{at} \mathrm{the} \mathrm{lowest} \mathrm{dose,} \mathrm{between} 46.3$ (week 6) and 61.6 (week 3) $\mu \mathrm{g} / \mathrm{kg}$ b.w./day at the mid dose and 400.3 (week 9) and 595.3 (week 2) $\mu \mathrm{g} / \mathrm{kg}$ b.w./day at the highest dose. The authors measured adipose tissue volume and liver fat content by magnetic resonance imaging (MRI). Further endpoints were cholesterol, 
triglycerides and apolipoprotein A-1a, changes in body weight and weight of the perirenal fat pad. There were no significant effects of BPA exposure on body weight or weight of the perirenal fat pad, and no differences were seen in total or visceral adipose tissue volumes between the groups. However, liver fat content was significantly higher in rats receiving the two higher doses of BPA compared with controls $(p=0.04)$. BPA exposure also increased the apolipoprotein A-I levels in plasma $(p<0.0001)$, a favourable modification in the lipid profile because apolipoprotein A-I is the main component of the high density lipoprotein (HDL).

\section{Conclusion on metabolic effects of BPA in animals}

Studies in rats and mice which were prenatally and postnatally exposed to BPA indicate that an effect on metabolic parameters was elicited, measured by effects on glucose or insulin or lipogenesis. The animal studies have shown an increase (Miyawaki et al., 2007; Rubin et al., 2001; Somm et al., 2009, Wei et al., 2011, Mackay et al., 2013), a decrease (Honma et al., 2002; Nagel et al., 1997), or no effect on body weight (Ryan et al., 2010, Marmugi et al., 2012; Mackay et al., 2013; Anderson et al., 2013) after early life exposure to BPA and the effect can occur in both or only one sex (Somm et al., 2009; Alonso-Magdalena et al., 2010; Mackay et al., 2013). The discrepancy among these animal studies probably arise from variation in experimental conditions, such as dosing regimes, animal species and strains, and timing of evaluation of effects. There is no consistent evidence that BPA is obesogenic in adulthood after intrauterine exposure or in longer-term studies. In some of the studies, the authors claimed that the findings indicate a non-monotonic dose-response as effects seen at a lower dose were not observed in higher doses. However, effects were seen only at one dose level and not in at least two dose levels with a decline at higher dose levels, which would be necessary to accept the existence of non-monotonicity.

\section{Epidemiological studies}

Some epidemiological studies also reported an association in adults of actual BPA levels in urine with cardiometabolic disorders (Lang et al., 2008, Melzer et al., 2010), or with obesity in children and adolescents (Trasande et al., 2012), but the validity of these results from the cross-sectional NHANES data were afterwards disputed (LaKind et al., 2012). Other epidemiological studies indicate inconsistent findings for an association between prenatal BPA exposure and a low birth weight, a predictor of obesity later in life (Lee et al., 2008; Miao et al., 2011; Padmanabham et al., 2008; Wolff et al., 2008). Hence, no firm conclusion can be drawn at present.

\section{$\underline{\text { In vitro studies }}$}

Several in vitro studies were published after 2010 reporting the effects of BPA on insulin secretion, mitochondrial morphology and function and gene expression in different cell types.

In the presence of low glucose concentrations, $3 \mathrm{mM}$ BPA had no effect (Soriano et al., 2012) Insulin secretion stimulated by high glucose levels $(8-17.7 \mathrm{M})$ was further increased by treatment with BPA concentrations $\left(10^{-10} \mathrm{M}, 10^{-9} \mathrm{M}\right.$ and $\left.2 \times 10^{-9} \mathrm{M}\right)$ in mouse and human islets, in primary rat islet cells and in a rat insulinoma cell line (Soriano et al., 2012; Song et al., 2012; Lin et al., 2013). These concentrations correspond to $22.8 \mathrm{ng} / \mathrm{L}$, $228 \mathrm{ng} / \mathrm{L}$ and $465 \mathrm{ng} / \mathrm{L}$, far above concentrations calculated in humans after oral exposure to BPA, which are in the $10^{-12} \mathrm{M}$ range (Teeguarden et al., 2013). The results from Soriano et al., (2012) suggest that BPA's effects on insulin secretion, KATP channel activity and glucose-induced [Ca2+] oscillations in pancreatic -cells are linked to the presence of ERß.

BPA-induced toxicity and apoptosis was associated with changes in the morphology and the membrane potential of mitochondria of pancreatic cells induced by BPA concentrations $\left(10^{-12} \mathrm{M}-10^{-8} \mathrm{M}\right.$ ) in the human hepatic cell line HepG2 (Huc et al., 2012). 
In isolated human adipose tissue taken from children, BPA at $10^{-8} \mathrm{M}$ increased the expression of $11 \beta$-hydroxysteroid-dehydrogenase, PPARa and lipoprotein lipase and, in addition, induced lipid droplet accumulation in adipocytes at terminal differentiation (Wang et al., 2013). Using transfection gene reporter assays with monkey kidney cells, Sheng and co-workers (2012) observed a BPA $\left(10^{-9} \mathrm{M}\right.$ to $\left.10^{-7} \mathrm{M}\right)$-induced suppression of thyroid hormone receptor transcription through a non-genomic pathway. However, the relevance of the model for the in vivo situation is unclear.

\section{Conclusions on metabolic activity}

Several studies in laboratory animals published in the last 5 years and more recently directly address the issue of whether developmental exposure to BPA can affect adiposity, glucose or insulin regulation, lipid profiles or other end-points related to diabetes or metabolic syndrome. Effects were increased body weight, elevated serum insulin, and impaired glucose tolerance in adult offspring. There is inconsistency in the results, as in some studies, effects were observed only in male offspring while in other studies, effects were only observed in female offspring. In addition, effects were not consistently induced by similar dosages and studies showing a lack of increase or even a decrease in body weight were reported. BPA exposure also affected various metabolic endpoints in adult rodents exposed to BPA. In some of the studies, the findings have been taken as evidence for a non-monotonic dose-response because effects seen at a lower dose were not observed at higher doses. However, effects were sometimes seen only at one dose level. There are no studies that demonstrate effects of different effect size at two consecutive dose levels and/or no or a reduced effect at a higher dose, thus corroborating the existence of non-monotonicity. In addition, epidemiological studies do not show unequivocal convincing evidence for metabolic effects of BPA. In view of the inconsistent results, no firm conclusions can be drawn at present, but the data warrant further assessment of the potential effects of BPA on adiposity, glucose or insulin regulation, lipids and other end-points related to diabetes or metabolic syndrome. However, as with other uncertain effects, EFSA did take into consideration possible effects on metabolic activity when performing the uncertainty analysis (EFSA 2015).

In vitro BPA was found to increase cellular insulin secretion only at high glucose levels, whereas at low glucose levels, a high BPA exposure was needed. In addition, lipid droplet accumulation was induced by BPA in human adipose tissues at relative high concentrations that could also induce cytotoxicity and apoptosis of the cells.

\subsubsection{Reproductive and developmental toxicity}

A host of studies is available on the effects of BPA on reproduction and prenatal development some of which were performed according to internationally agreed guidelines and in compliance with GLP. A wealth of in vitro results and studies on nonintact animals (such as ovariectomized rodents) is available, but their value for risk assessment is questionable. There are also uncertainties as to reproducibility of several individual studies. The text below focuses on GLP-compliant guideline-based generation and developmental studies. These studies were conducted in rats and mice. Chapin et al. (2008) performed a comprehensive review of all available animal studies. A selection of studies critical for the determination of reproductive and developmental toxicity and the derivation of NOAELs is revisited below.

Morrissey et al. (1987) studied the developmental toxicity of BPA in CD rats $(0,160,320$, or $640 \mathrm{mg} / \mathrm{kg} \mathrm{b.w./day)} \mathrm{and} \mathrm{CD-1} \mathrm{mice}(0,500,750,1000$, or $1250 \mathrm{mg} / \mathrm{kg}$ b.w./day) dosed daily by gastric intubation on Gestational Days 6 through 15. In rats, maternal weight gains during gestation, weight gain corrected for gravid uterine weight and weight gain during treatment were significantly reduced at all BPA doses. Gravid uterine weight and average foetal body weight per litter were not affected by BPA. No increase in percentage resorptions per litter or percentage foetuses malformed per litter was 
detected. In mice, maternal mortality occurred at all BPA doses, reaching $18 \%$ at the high dose, which also produced a significant decrease in maternal body weight gain during gestation and treatment. Weight gain corrected for gravid uterine weight was not affected by BPA. Reductions in gravid uterine weight and average foetal body weight were observed with the $1250 \mathrm{mg} / \mathrm{kg}$ dose of BPA. Relative maternal liver weight was increased at all doses of BPA. There was a significant increase in the percentage of resorptions per litter with $1250 \mathrm{mg} \mathrm{BPA} / \mathrm{kg}$ b.w./day. Malformation incidence was not altered by BPA. Thus, BPA treatment at maternally toxic dose levels during organogenesis produced foetal toxicity in mice but not in rats and did not alter foetal morphologic development in either species.

Ema et al. (2001) conducted a two-generation study in Crj:CD(SD) IGS rats using doses of $0.2,2,20$ and $200 \mu \mathrm{g} / \mathrm{kg}$ b.w./day by oral gavage, starting exposure 10 and 2 weeks before mating in males and females, respectively. No compound-related clinical signs or effects on body weight or food consumption were observed in any generation. There were no compound-related changes in surface righting reflex, negative geotaxis reflex, mid-air righting reflex, pinna detachment, incisor eruption, eye opening, testes descent, preputial separation, or vaginal opening in F1 and F2 generations, or behavior in the open field or water filled multiple T-maze tests in the F1 generation. No test compoundrelated changes in estrous cyclicity, copulation index, fertility index, number of implantations, gestation length, litter size, pup weight, pup sex ratio, pup viability, or other functional reproductive measures were noted in any generation. A few significant changes in the anogenital distance (AGD) per cube root of body weight ratio were found at 0.2 and $20 \mu \mathrm{g} / \mathrm{kg} \mathrm{b.w.} \mathrm{in} \mathrm{F1} \mathrm{males,} \mathrm{at} \mathrm{2,} \mathrm{20,} \mathrm{and} 200 \mu \mathrm{g} / \mathrm{kg}$ b.w. in F1 females and at 20 and $200 \mu \mathrm{g} / \mathrm{kg} \mathrm{b.w.} \mathrm{in} \mathrm{F2} \mathrm{females.} \mathrm{However,} \mathrm{the} \mathrm{changes} \mathrm{in} \mathrm{the} \mathrm{AGD} \mathrm{were}$ consistently small (within $5 \%$ of control values), and no continuous changes in the AGD or AGD/cube root of body weight ratio were detected. There were no compound-related changes in epididymal sperm counts or motility in F0 and F1 males. No compoundrelated necropsy findings or effects on organ weight, including the reproductive organs, were found in any generation. Histopathologic examinations revealed no evidence of compound-related changes in any organs including the reproductive organs of both sexes. The data indicate that oral doses of BPA of between 0.2 and $200 \mu \mathrm{g} / \mathrm{kg} \mathrm{b} . \mathrm{w}$. over two generations did not cause significant compound-related changes in reproductive or developmental parameters in rats.

Tyl et al. (2002) performed a three-generation study in Sprague Dawley rats using a wide range of dietary doses of 0.001 to $500 \mathrm{mg} / \mathrm{kg} \mathrm{b.w./day.} \mathrm{Adult} \mathrm{systemic} \mathrm{toxicity}$ occurred at $50 \mathrm{mg} / \mathrm{kg}$ b.w./day including reduced body and organ weight gain, with hepatic pathology at $500 \mathrm{mg} / \mathrm{kg} /$ day. Ovarian weights as well as total pups and live pups/litter on postnatal day (PND) 0 were decreased at $500 \mathrm{mg} / \mathrm{kg}$ b.w./day, which exceeded the adult maximum tolerated dose (MTD). Mating, fertility, gestational indices; ovarian primordial follicle counts; estrous cyclicity; precoital interval; gestational length; offspring sex ratios; postnatal survival; nipple/areolae retention in preweanling males; epididymal sperm number, motility, morphology; daily sperm production (DSP), and efficiency of DSP were all unaffected. Adult systemic toxicity no observed adverse effect level (NOAEL) was $5 \mathrm{mg} / \mathrm{kg}$ b.w./day; reproductive and postnatal NOAEL was 50 $\mathrm{mg} / \mathrm{kg} / \mathrm{day}$. There were no treatment-related effects in the low-dose region $(0.001-5$ $\mathrm{mg} / \mathrm{kg}$ b.w./day) on any parameters and no evidence of nonmonotonic dose-response curves across generations for either sex. The authors conclude that BPA should not be considered a selective reproductive toxicant, based on the results of this study.

Kobayashi et al. (2002) exposed Crj:CD(SD) IGS rat dams to 0,4 or $40 \mathrm{mg} / \mathrm{kg} / \mathrm{day}$ from GD6 through PND20. There were no significant changes in body weight, liver weight, kidneys weight, testes weight, AGD, the ratio of AGD to body weight, or the ratio of AGD to the cube root of body weight in BPA exposed pups compared to the vehicle-exposed control. The authors conclude that prenatal and postnatal exposure (indirect exposure) to BPA (4- $40 \mathrm{mg} / \mathrm{kg}$ b.w./day, GD 6-PND 20) does not affect somatic growth or AGD of F1 generation of male and female rats. 
Tyl et al. (2008) conducted a two-generation study of BPA in CD-1 mice. F0 and F1 mice (28 sex/group/generation) were fed diets containing BPA (99.70-99.76\% purity) at $0.018,0.18,1.8,30,300$, or $3500 \mathrm{ppm}$. Target intakes were $0.003,0.03,0.3,5,50$, or $600 \mathrm{mg} / \mathrm{kg}$ b.w./day, respectively. A concurrent positive control group of dietary 17betaestradiol ( 0.5 ppm; 28 per sex) confirmed the sensitivity of $C D-1$ mice to an endogenous estrogen. There were no clinical signs of toxicity or treatment-related deaths in F0 or F1 males or females. Increases in weight, both absolute and relative to body or brain, of the kidney and liver were consistently observed in F0 and F1 adults (males and females) at the highest dose level. At $50 \mathrm{mg} / \mathrm{kg} \mathrm{b.w./day} \mathrm{hepatocyte} \mathrm{hypertrophy} \mathrm{was} \mathrm{noted} \mathrm{and} \mathrm{at}$ $600 \mathrm{mg} / \mathrm{kg} \mathrm{b.w./day} \mathrm{body} \mathrm{and} \mathrm{organ} \mathrm{weight} \mathrm{effects} \mathrm{were} \mathrm{also} \mathrm{observed.} \mathrm{Incidence} \mathrm{of}$ minimal to mild hepatocyte centrilobular hypertrophy was increased in both generations at 300 and/or 3500 ppm. Renal nephropathy incidence was increased in F0 males and in F1 males and females of the 3500 ppm group.

There were no BPA-related effects on adult mating, fertility or gestational indices, ovarian primordial follicle counts, estrous cyclicity, precoital interval, offspring sex ratios or postnatal survival, sperm parameters or reproductive organ weights or histopathology (including the testes and prostate). BPA exposure had no effect on numbers of implantation sitesor resorptions or on mating, fertility, or gestational indices in F0 or F1 mice. Gestational length was increased in F0 and F1 females from the 3500 ppm group; the study authors stated the effect was of unknown biological significance. Epididymal sperm concentration was decreased in F0 males of the 3500 ppm group, but no effect was observed in F1 parental or retained males. There was no effect on daily sperm production, efficiency of daily sperm production, or sperm motility or morphology in either generation. The study authors did not consider the decrease in sperm concentration in FO animals to be treatment-related, based on lack of consistency between generations, no detectable effect on any other andrological endpoint, and no detectable effect on fertility. Estrous cyclicity and numbers of ovarian primordial follicle counts were not affected by BPA exposure in FO or F1 females. The only gross observation in reproductive organs was a slightly increased incidence of gross ovarian cysts in F0 females from the 3500 ppm group. The incidence of paraovarian cysts was increased in F0 and F1 females from the 3500 ppm group. In F1 pups from the 3500 ppm group, body weights were reduced during PND 7, 14, and 21 in F1 females and both sexes combined and on PND 7 and 21 in F1 males. Preputial separation (absolute age and adjusted for body weight on day of acquisition) was delayed in $\mathrm{F} 1$ males of the 3500 ppm group. The study authors reported no gross findings in F1 or F2 weanlings. The incidence of undescended bilateral testes was increased in F1 and F2 weanling males of the 3500 ppm group. The incidence of hepatic cytoplasm alteration (clear hepatocellular cytoplasm, slightly more basophilic cytoplasm, and/or minute vacuoles) was apparently increased in F1 males from the 300 and 3500 ppm groups and F1 females and F2 males from the $3500 \mathrm{ppm}$ group. The incidence of seminiferous tubule hypoplasia was increased in F1 and F2 weanlings from the $3500 \mathrm{ppm}$ group. The study authors identified bisphenol A NOELs of $30 \mathrm{ppm}$ ( $5 \mathrm{mg} / \mathrm{kg}$ b.w./day) for systemic effects, $300 \mathrm{ppm}$ ( $50 \mathrm{mg} / \mathrm{kg}$ b.w./day) for developmental toxicity, and $300 \mathrm{ppm}(50 \mathrm{mg} / \mathrm{kg} \mathrm{b.w./day)} \mathrm{for} \mathrm{reproductive}$ toxicity. Therefore, BPA was not considered a selective reproductive or developmental toxicant in mice.

Kobayashi et al. (2010) exposed C57BL/6J mice to dietary levels of $0.33,3.3$ or $33 \mathrm{ppm}$ BPA. from GD6 through PND22, and the weanlings $(F(1)$ and $F(2)$ ) from each $F(0)$ and $F(1)$ dam group, respectively, dosing was continued until sacrifice. There were no treatment-related changes in body weight, body weight gain, food consumption, gestation length, or the number of live births on postnatal day 1 in $F(0)$ dams between the control group and BPA groups. Sex ratio and viability were similar in all $F(1)$ pups. No treatment-related changes were observed in body weight, food consumption, developmental parameters, anogenital distance, or weight of any of the organs (liver, kidney, heart, spleen, thymus, testis, ovary, or uterus) in $F(1)$ and $F(2)$ adults in either sex. The epididymis weight was slightly higher with 0.33 and $3.3 \mathrm{ppm}$ in $F(1)$ males, but this slight increase was neither dose dependent nor seen across generations. There were 
no treatment-related effects of BPA on cauda epididymal sperm count or sperm motility in $F(1)$ or $F(2)$ males. These findings indicate that dietary exposure to bisphenol $A$ between 0.33 and $33 \mathrm{ppm}$ does not adversely affect reproduction or development as assessed in two generations of mice.

Stump et al. (2010) conducted a developmental neurotoxicity study in Crl:CD(SD) rats with dietary concentrations of $0.15,1.5,75,750$, and $2250 \mathrm{ppm}$ daily from gestation day 0 through lactation day 21 . $F(1)$ offspring were evaluated using the following tests: detailed clinical observations (PNDs 4, 11, 21, 35, 45, and 60), auditory startle (PNDs 20 and 60), motor activity (PNDs 13,17, 21, and 61), learning and memory using the Biel water maze (PNDs 22 and 62), and brain and nervous system neuropathology and brain morphometry (PNDs 21 and 72). For $F(1)$ offspring, there were no treatment-related neurobehavioral effects, nor was there evidence of neuropathology or effects on brain morphometry. Based on maternal and offspring body weight reductions, the noobserved-adverse-effect level (NOAEL) for systemic toxicity was $75 \mathrm{ppm}$ (5.85 and 13.1 $\mathrm{mg} / \mathrm{kg} /$ day during gestation and lactation, respectively), with no treatment-related effects at lower doses or nonmonotonic dose-responses observed for any parameter. There was no evidence that BPA is a developmental neurotoxicant in rats, and the NOAEL for developmental neurotoxicity was $2250 \mathrm{ppm}$, the highest dose tested (164 and 410 $\mathrm{mg} / \mathrm{kg} /$ day during gestation and lactation, respectively).

In a preliminary study with two doses $(10 \mu \mathrm{g} / \mathrm{kg} \mathrm{b.w./day} \mathrm{and} 5 \mathrm{mg} / \mathrm{kg} \mathrm{b.w./day)} \mathrm{in} \mathrm{male}$ rats, oral BPA by gavage for 6 days induced for both doses a decrease in sperm production (Tiwari and Vanage 2013).

Recently Delclos et al. (2014) published the results of a toxicity study of BPA using a low dose range $(2.5-2700 \mu \mathrm{g} / \mathrm{kg} \mathrm{b.w./day,} \mathrm{with} \mathrm{a} \mathrm{total} \mathrm{of} 7$ doses within this range) below the current NOAEL of $5,000 \mu \mathrm{g} / \mathrm{kg}$ b.w./day and two very high $(100,000-300,0000$ $\mu \mathrm{g} / \mathrm{kg}$ b.w./day) doses of BPA. The dosing was done by gavage and started at gestational day 6 and lasted until postnatal day 90 . The effects of BPA were compared to ethinyl estradiol (EE2) $(0.5$ and $5.0 \mu \mathrm{g} / \mathrm{kg}$ b.w./day) to evaluate estrogen responsiveness. The low doses should provide insight in possible low dose endocrine disrupting effects, similar to the estrogenic ethinyl estradiol, whereas the high doses were chosen because of expected toxicity. The focus of the study was reproductive toxicity but other parameters (e.g. serum glucose, insulin, histopathology of heart and blood vessels) were evaluated as well. Only some of the main observations are summarized here. Only for the high doses investigated clear adverse effects were noted being depressed gestational and postnatal body weight gain, effects on the ovary (increased cystic follicles, depleted corpora lutea, and antral follicles), and serum hormones (increased serum estradiol and prolactin and decreased progesterone). Some of these effects were also observed for ethinyl estradiol the positive estrogenic control in this study. Only for the highest BPA dose $(300,0000 \mu \mathrm{g} / \mathrm{kg}$ b.w./day) did preweaning pup survival decrease, and body weights of both females and males were lower compared to control groups. For the $100,0000 \mu \mathrm{g} / \mathrm{kg}$ b.w./day BPA dose group lower body weights were only observed for the females. In contrast, EE2 induced an increase in female body weight. Regarding markers of sexual development, none of the BPA doses affected vaginal opening which was delayed after EE2 treatment, testicular descend was delayed for $1-2$ days in the 260 and $300,0000 \mu \mathrm{g} / \mathrm{kg}$ b.w./day BPA groups and for 5.8 days the $5 \mu \mathrm{g} / \mathrm{kg} \mathrm{b} . w . /$ day EE2 group, while preputial separation was only delayed in the $5 \mu \mathrm{g} / \mathrm{kg}$ b.w./day EE2 group. Female estrous cyclus was only affected by the highest dose BPA $(300,000 \mu \mathrm{g} / \mathrm{kg}$ b.w./day) and in both EE2 ( 0.5 and $5 \mu \mathrm{g} / \mathrm{kg}$ b.w./day) dose groups as indicated by vaginal cytology and histopathology. Serum estradiol levels in females were increased in both high BPA dose groups and the EE2 treated groups, but not in the low dose BPA groups. In males, there were no treatment-related differences between any treatment group and vehicle control for the measured hormones $\mathrm{FSH}, \mathrm{LH}$, testosterone, and prolactin. In the study other additional external potential sources of BPA exposure (e.g. animal bedding, polysulfone cages, silicone water bottle stoppers and drinking water) were evaluated for BPA leakage of which none was detectable above analytical blanks. Also phytoestrogen presence in feed was monitored and limited by using a soy- and 
alfafa free diet. However, based on BPA measurements and/or BPA-glucuronide conjugates in serum, the control animals had an unintentional exposure similar to the 2.5 $\mu \mathrm{g} / \mathrm{kg}$ b.w./day group the lowest BPA dose group (Churchwell et al., 2014). The authors concluded that "the results of the present study show that BPA in the low dose region from 2.5 to $2700 \mu \mathrm{g} / \mathrm{kg} \mathrm{b.w./day} \mathrm{did} \mathrm{not} \mathrm{produce} \mathrm{effects} \mathrm{in} \mathrm{the} \mathrm{evaluated} \mathrm{endpoints} \mathrm{that}$ differ from normal background biological variation. The experimental model was sufficiently sensitive to detect clear effects of EE2 at both dose levels tested, as well as of the high BPA doses". As the estrogenic adverse effects were only seen at the high dose exposures, this indicates and confirms the weak estrogenic activity of BPA. In the study low dose effects for doses below the current NOAEL of 5,000 $\mu \mathrm{g} / \mathrm{kg} \mathrm{b.w./day,} \mathrm{associated}$ with reproductive parameters and/or estrogenic activity, could not be detected.

\section{Conclusions}

Overall, female reproductive toxicity occurred with an overall NOAEL of $50 \mathrm{mg} / \mathrm{kg}$ b.w./day and a LOAEL of $500 \mathrm{mg} / \mathrm{kg}$ b.w./day, derived from the Tyl et al. (2002) multigeneration study. However, at the LOAEL for female reproductive effects, significant body weight reduction and hepatic toxicity occurred. As to developmental toxicity, BPA does not cause malformations or birth defects in rats or mice at levels up to the highest doses evaluated: $640 \mathrm{mg} / \mathrm{kg}$ b.w./day (rats) and $1250 \mathrm{mg} / \mathrm{kg} \mathrm{b.w./day} \mathrm{(mice)} \mathrm{(Morrissey}$ et al., 1987). BPA does not alter male or female fertility after gestational exposure up to doses of $500 \mathrm{mg} / \mathrm{kg} \mathrm{b.w./day} \mathrm{in} \mathrm{the} \mathrm{rat} \mathrm{(Tyl} \mathrm{et} \mathrm{al.,} \mathrm{2002)} \mathrm{and} 600 \mathrm{mg} / \mathrm{kg} \mathrm{b.w./day} \mathrm{in} \mathrm{the}$ mouse (Tyl et al., 2008), being highest dose levels evaluated. BPA does not permanently affect prostate weight at doses up to $475 \mathrm{mg} / \mathrm{kg} \mathrm{b.w./day} \mathrm{in} \mathrm{adult} \mathrm{rats} \mathrm{or} 600 \mathrm{mg} / \mathrm{kg}$ b.w./day in mice. BPA does change the age of puberty in male or female rats at high doses (ca. $500 \mathrm{mg} / \mathrm{kg}$ b.w./day). Neurodevelopmental toxicity was not observed at the highest dose tested (164/410 mg/kg b.w./day, Stump et al., 2010). In a recent study by Delclos et al., (2014) low dose effects for doses below the current NOAEL of 5,000 $\mathrm{gg} / \mathrm{kg}$ b.w./day, associated with reproductive parameters and/or estrogenic activity, could not be detected.

Uncertainty regarding reproductive and developmental toxicity was large at the lower end of the investigated dose range, and therefore, it was not taken forward for risk characterisation in the recent EFSA evaluation (EFSA 2015). Nevertheless, the effects reported at the lower end of the dose range were included in the uncertainty evaluation by EFSA.

On the basis of the above studies, it can be concluded that reproductive or developmental toxicity are not the critical end-point in BPA toxicity, although it does have reproductive toxicity at doses higher than those causing liver and kidney damage. On this base it was classified as a Repro 2 toxicant (being classification an hazard-based rather than a risk-based process). More recently, the Committee for Risk Assessment (RAC) of the European Chemicals Agency (ECHA) has adopted an opinion to strengthen the existing harmonised classification and labelling (CLH) of BPA from a category 2 reproductive toxicant to a category $1 \mathrm{~B}$ reproductive toxicant regarding the adverse effects on sexual function and fertility in line with a proposal from the French competent authority (ECHA 2014).

\subsubsection{Conclusions on toxicity}

\section{General toxicity}

The key studies for setting a NOAEL were considered to be the multi-generation studies in rats and mice performed by Tyl et al. investigating the general and reproductive toxicity of BPA over a wide range of oral doses (Tyl et al., 2002, 2008). Based on these studies, a NOAEL of $5 \mathrm{mg} / \mathrm{kg}$ b.w./day was derived based on liver toxicity, whereas the NOAEL for reproductive toxicity was set at $50 \mathrm{mg} / \mathrm{kg} \mathrm{b.w./day.} \mathrm{In} \mathrm{these} \mathrm{multi-generation}$ studies in rats and mice, alterations in kidney weight were also observed at the low 
administered doses, but renal weight increased in mice and decreased in rats, although the relative kidney weight also increased in rats. For the higher doses investigated in both mice and rats, histopathological alterations were noted in the kidneys (Tyl et al., 2002, 2008). Using the results of Tyl et al. (2002, 2008), EFSA (EFSA 2014, 2015) has recently applied a BMD approach (EFSA 2009, 2011) for further refining the risk assessment with kidney weight as the critical endpoint.

With the $B M D$ approach a confidence interval $\left(B M D L_{10}-B M D U_{10}\right)$ of the $B M D_{10}$, being a dose resulting in a $10 \%$ deviation from vehicle treated control animals, was obtained. $\mathrm{BMDL}_{10}$ indicates the lower part $(5 \%)$ of the $90 \%$ confidence interval and $\mathrm{BMDU}_{10}$ indicates the upper part $(95 \%)$ of the confidence interval. A BMDL $L_{10}$ of $3420 \mu \mathrm{g} / \mathrm{kg}$ b.w./day, and a $\mathrm{BMDU}_{10}$ of $91690 \mu \mathrm{g} / \mathrm{kg}$ b.w./day was calculated for changes in male mouse absolute kidney weight by EFSA (2015) based on Tyl et al. (2008). For the absolute kidney weights wide confidence intervals were obtained because of lack of dose response information in the data. Considering the dependence of organ weight on body weight, EFSA also evaluated the relative kidney weights for which adequate dose response information was obtained. In addition, the confidence intervals for the BMD calculations were smaller for the relative organ weights, indicating better dose-response relationships and less variability in the data for relative organ weights (EFSA 2015). For the risk assessment, EFSA established a BMDL ${ }_{10}$ of $8960 \mu \mathrm{g} / \mathrm{kg} \mathrm{b.w./day} \mathrm{with} \mathrm{the} \mathrm{relative}$ kidney weight as the critical endpoint. The $\mathrm{BMDL}_{10}$ of $8.96 \mathrm{mg} / \mathrm{kg} \mathrm{b.w./day} \mathrm{derived} \mathrm{by}$ EFSAis very close to the NOAEL of $5 \mathrm{mg} / \mathrm{kg} \mathrm{b.w./day} \mathrm{as} \mathrm{determined} \mathrm{in} \mathrm{the} \mathrm{Tyl} \mathrm{et} \mathrm{al.}$ (2008) study.

For BPA, the two derived values (NOAEL and $\mathrm{BMDL}_{10}$ ) are conceptually different from a toxicokinetic point of view. Indeed, the internal exposure of the organs is different: the hepatic exposure is presystemic, whereas the renal one is systemic. The doses at the site of action (i.e. liver and kidney) differ after the oral route of exposure, because of the biotransformation occurring in the liver resulting in a lower dose of free BPA for kidney exposure. The SCENIHR supports the use of the kidney effects as the PoD and the newly developed t-TDI for the risk assessment of medical devices.

\section{Genotoxicity}

BPA was found not to interact with DNA directly as it does not induce gene mutation in bacteria (Masuda et al., 2005; Tiwari et al., 2012) and micronuclei in vivo in rodent erythropoietic cells (Masuda et al., 2005; Pacchierotti et al., 2008; De Flora et al., 2011, Naik et al., 2009). There was also no induction of chromosomal aberrations observed in bone marrow cells of mice treated in vivo with BPA (Naik et al., 2009), however, BPA acts on the mitotic spindle apparatus. The large margin between the dose-levels found negative in vivo for induction of aneuploidy in rodent germ cells (Pacchierotti et al., 2008) and for induction of micronuclei in somatic bone marrow cells (Masuda et al., 2005; Pacchierotti et al., 2008; Naik et al., 2009; De Flora et al., 2011) provides adequate reassurance on the lack of aneugenic effects of BPA in vivo. So, BPA is not likely to pose a genotoxic hazard to humans.

\section{Carcinogenicity}

From all the study results taken together, it can be concluded that in standard carcinogenic testing protocols according to OECD BPA has no carcinogenic activity. In additional multigeneration studies (Tyl et al., 2002; Tyl et al., 2008), no indication of increased cancerogenicity was observed; in particular, preneoplatic lesions of the mammary gland were absent in all offspring. In contrast, three studies from the same group in rats have demonstrated an effect of prenatal BPA exposure on mammary gland development, i.e. ductal hyperplasia, and one study showed some carcinoma development. These studies were performed with very low background estrogen levels in the feed which may have had an effect on the normal development in the controls. A limitation is the lack of a positive control like estradiol for comparing the estrogenic effects induced by BPA. Similar effects were indicated in studies in mice and rhesus monkeys, supporting the observations in rats. Therefore, it cannot be excluded that BPA 
affects early development of mammary tissue, although their relevance for adversity in humans is not clear. These studies should, therefore, be considered as an indicator for a possible concern.

Studies using s.c. administration of BPA indicated that BPA may have the ability to increase the effects of well-known carcinogens even at very low BPA levels. The studies had limitations that render them unsuitable to assess whether BPA has a carcinogenic potential by prenatal or peri-natal exposure. The main limitation is that in the studies with positive outcome additional treatment with a strong initiating or additional promoting agent(s) has been performed. Furthermore, in most of the studies the statistical analysis does not consider litter effects, and in addition, multiple statistical testing has been performed without proper adjustment to avoid positive results by chance. Further studies were performed in transgenic animals, the results of which can not be extrapolated directly to the human situation.

The current situation shows no carcinogenic effects in OECD guideline studies. However, studies indicating effects on mammary gland development raise some concern for a possible effect late in life after prenatal exposure.

\section{Reproductive toxicity}

Overall, female reproductive toxicity occurred with an overall NOAEL of $50 \mathrm{mg} / \mathrm{kg}$ b.w./day and a LOAEL of $500 \mathrm{mg} / \mathrm{kg} \mathrm{b.w./day,} \mathrm{derived} \mathrm{from} \mathrm{the} \mathrm{Tyl} \mathrm{et} \mathrm{al.} \mathrm{(2002)}$ multigeneration study. However, at the LOAEL for female reproductive effects significant body weight reduction and hepatic toxicity occurred. As to developmental toxicity, Bisphenol A does not cause malformations or birth defects in rats or mice at levels up to the highest doses evaluated: $640 \mathrm{mg} / \mathrm{kg}$ b.w./day (rats) and $1250 \mathrm{mg} / \mathrm{kg} \mathrm{b.w./day}$ (mice) (Morrissey et al., 1987). BPA does not alter male or female fertility after gestational exposure up to doses of $500 \mathrm{mg} / \mathrm{kg} \mathrm{b.w./day} \mathrm{in} \mathrm{the} \mathrm{rat} \mathrm{(Tyl} \mathrm{et} \mathrm{al.,} \mathrm{2002)} \mathrm{and}$ $600 \mathrm{mg} / \mathrm{kg} \mathrm{b.w./day} \mathrm{in} \mathrm{the} \mathrm{mouse} \mathrm{(Tyl} \mathrm{et} \mathrm{al.,} \mathrm{2008,} \mathrm{highest} \mathrm{dose} \mathrm{levels} \mathrm{evaluated).} \mathrm{BPA}$ does not permanently affect prostate weight at doses up to $475 \mathrm{mg} / \mathrm{kg} \mathrm{b.w./day} \mathrm{in} \mathrm{adult}$ rats or $600 \mathrm{mg} / \mathrm{kg} \mathrm{b.w./day} \mathrm{in} \mathrm{mice.} \mathrm{BPA} \mathrm{does} \mathrm{change} \mathrm{the} \mathrm{age} \mathrm{of} \mathrm{puberty} \mathrm{in} \mathrm{male} \mathrm{or}$ female rats at high doses (ca. $500 \mathrm{mg} / \mathrm{kg}$ b.w./day). Neurodevelopmental toxicity was not observed at the highest dose tested (164/410 mg/kg b.w./day, Stump et al., 2010). In the recent study by Delclos et al. (2014), low dose effects for doses below the current NOAEL of $5,000 \mu \mathrm{g} / \mathrm{kg}$ b.w./day, associated with reproductive parameters and/or estrogenic activity, could not be detected.

On the basis of the above studies, it can be concluded that reproductive or developmental toxicity are not the critical end-point in BPA toxicity: general toxicity effects such as body and organ weights and liver histopathology and nephropathy often occur simultaneously with reproductive or developmental effects, which are only observed at high dosages. On this base it was classified as a Repro 2 toxicant (being classification an hazard-based process). More recently, The Committee for Risk Assessment (RAC) of the European Chemicals Agency (ECHA) has adopted an opinion to strengthen the existing harmonised classification and labelling (CLH) of BPA from a category 2 reproductive toxicant to a category $1 \mathrm{~B}$ reproductive toxicant regarding the adverse effects on sexual function and fertility in line with a proposal from the French competent authority (ECHA, 2014).

\section{Immunotoxicity}

BPA is able to elicit skin sensitization in humans probably because it is a weak sensitizer. Studies on a possible relationship between prenatal and/or postnatal BPA exposure and allergic responses are not consistent. Other studies on immunotoxic responses are insufficient for drawing final conclusions.

\section{Metabolic effects}

Several studies in laboratory animals published in the last 5 years and more recently directly addressed whether developmental exposure to BPA can affect adiposity, glucose or insulin regulation, lipid profiles or other end-points related to diabetes or metabolic 
syndrome. Effects were increased body weight, elevated serum insulin and impaired glucose tolerance in adult offspring. There is inconsistency in the results as effects were observed in some studies only in male offspring while in other studies effects were only observed in female offspring. In addition, effects were not consistently induced by similar dosages. BPA exposure also affected various metabolic endpoint in adult rodents exposed to BPA. In some of the studies, the findings could be taken as evidencing a nonmonotonic dose-response as effects were seen at a lower dose which was not observed in higher doses. However, effects were seen only at one dose level. There are no studies that demonstrate effects of different effect size at two dose levels and no or a reduced effect at a higher dose, thus corroborating the existence of non-monotonicity. Additionally, epidemiological studies do not show unequivocally conclusive evidence for metabolic effects of BPA. Although no firm conclusion can be drawn at present, the data warrant further assessment of the potential effects of BPA on adiposity, glucose or insulin regulation, lipids and other end-points related to diabetes or metabolic syndrome.

In vitro BPA was found to increase cellular insulin secretion only at high glucose levels, whereas at low glucose levels a high BPA exposure was needed. In addition, lipid droplet accumulation was induced by BPA in human adipose tissues at relative high concentrations. These high concentrations could also induce cytotoxicity and apoptosis of the cells.

BPA is a chemical that has been investigated in studies performed according to OECD protocols and compliant to GLP principles. The array of studies performed is such as requested for a high production volume chemical according to the REACH legislation plus one neurotoxicity study as requested for pesticides. In these studies, the lowest NOAEL ( $5 \mathrm{mg} / \mathrm{kg} \mathrm{b.w./day} \mathrm{orally)} \mathrm{was} \mathrm{found} \mathrm{in} \mathrm{a} \mathrm{developmental} \mathrm{study} \mathrm{in} \mathrm{rats} \mathrm{(Tyl} \mathrm{et} \mathrm{al.,} \mathrm{2002)}$ as an effect on the liver. Centrilobular hepatocyte hypertrophy was also seen in the 3generation study in mice (Tyl et al., 2008) at an oral dose of $50 \mathrm{mg} / \mathrm{kg} \mathrm{b.w./day.} \mathrm{Other}$ effects in the same study were increased kidney weight and nephropathy. No developmental and no fertility endpoint other than reduced sperm concentration at the highest dose was changed in this study which investigated oral doses of $0.003,0.03,0.3$, 5,50 , or $600 \mathrm{mg} \mathrm{BPA} / \mathrm{kg}$ b.w./day. No carcinogenicity was observed in a classical NTP study. The results of gentoxicity testing were grossly negative. Testing for immuntoxicity revealed some effects; however, without indicating that BPA is clearly an immuntoxic substance. New studies with "untypical" endpoints have raised concerns. The endpoints encompass cell proliferation in the male and femal breast after intrauterine and postnatal exposure, anxiety and behavioural endpoints after intrauterine and postnatal exposure and changes in metabolic endpoints also after intrauterine and postnatal exposure.

\section{Overall conclusions}

In conclusion, there are several indications that BPA has biological effects below the current NOAEL of $5 \mathrm{mg} / \mathrm{kg}$ b.w./day and the recently established $\mathrm{BMDL}_{10}$ of $8.96 \mathrm{mg} / \mathrm{kg}$ b.w./day (oral repeated exposure). However, the evidence has been mainly obtained in dedicated studies focussing on specific outcome parameters like adiposity and hormone levels, and not in general toxicity studies. Some of those parameters resulted in contradicting results in various studies (e.g. decrease, no effect or increase in body weight). In addition, dose-response relationships could not be established. However, those effects were taken into consideratiuon in the uncertainty analysis by EFSA (EFSA, 2015).

\subsection{Epidemiological studies}

There are a limited but increasing number of epidemiological studies that investigated an association between BPA exposure and health outcomes. Most of them use crosssectional designs that limit their interpretability, especially for outcomes that have long latency periods (e.g. cardiovascular disease, diabetes). Frequently, a single measure of 
urinary BPA is used to categorize exposure, which given the short half-life of BPA, is another limitation. Fortunately, many of those epidemiological studies utilized the same laboratory at the United States Centers for Disease Control and Prevention for quantification of urinary BPA concentrations (Wolff et al., 2007, 2008b, 2010; Lang et al., 2008; Braun et al., 2009; Mok-Lin et al., 2009; Meeker et al., 2010a; Meeker et al., 2010b; Melzer et al., 2010, 2011, 2012; Mendiola et al., 2010; Clayton et al., 2011; Carwile and Michels 2011; Silver et al., 2011; and Shankar et al., 2012a,b,c). This eliminates one potential source of variability in comparisons across these studies.

We have identified 34 peer-reviewed epidemiological studies that examined associations between BPA exposure and human health outcomes.

Occupational and non-occupational exposure to BPA was investigated in relation with some reproductive outcomes, including serum sex steroid hormone concentrations, semen quality, oocyte count, recurrent miscarriage, endometriosis, endometrial hyperplasia and cancer, and polycystic ovarian syndrome (Hanaoka et al., 2002; Hiroi et al., 2004; Takeuchi et al., 2004; Sugiura-Ogasawara et al., 2005; Itoh et al., 2007; Cobellis et al., 2009; Mok-Lin et al., 2009; Galloway et al., 2010; Li et al., 2010a,b,c; Meeker et al., 2010a; Meeker et al., 2010b; Mendiola et al., 2010; Bloom et al., 2011 and Hao et al., 2011).

Interestingly, three epidemiological studies investigated the association of urinary BPA concentrations with semen quality. Men who were partners of pregnant women in the USA (Mendiola et al., 2010), male partners in infertile couples who were patients in an infertility clinic (Meeker et al., 2010) and workers with occupational exposure to BPA in China (Li et al., 2010c). Although all three studies, with a relatively modest sample size (ranging from 190 to 302 men), reported associations of increased urinary BPA concentration and one or more measures of reduced semen quality, this was statistically significant in only one of them. Limitations include their cross-sectional designs and incomplete assessment of occupational co-exposure in one of the three studies.

The evidence for an association of BPA with altered age of pubertal onset in girls was investigated in two epidemiological studies, a cross-sectional study in New York City, New York, USA (Wolff et al., 2008a) and in a prospective cohort study of 1151 female children from Cincinnati, Ohio, San Francisco, California, and New York City, New York, USA (Wolff et al., 2008a, 2010). Results are limited and inconsistent. Research needs to include large prospective studies on the association of BPA with pubertal development. A research gap is the lack of studies on male pubertal development in relation to BPA exposure.

Only one study examined the association between BPA exposure and cancer (Yang et al., 2009). In a breast cancer case-control study of 152 women, serum samples were used to quantify BPA exposure. Although cases had higher median serum BPA concentrations than controls, differences were not statistically different. Because BPA has a short halflife, current serum BPA concentrations may not be relevant to the etiological window of development for breast cancer, which is years to decades before clinical recognition.

It is difficult to draw any conclusions from two published epidemiological studies that have examined the association of BPA with perinatal outcomes because contradictory results. Six published epidemiological studies have examined the association of BPA with perinatal outcomes, body mass index and neurodevelopment. Two studies examining perinatal outcomes relied on a single serum measure of BPA at birth (Padmanabhan et al., 2008; Chou et al., 2011); others relied on a single urinary BPA concentration during pregnancy (Wolff et al., 2008b; Philippat et al., 2012), another estimated BPA exposure by personal air sampling measurements and exposure history. Wolff and colleagues (Wolff et al., 2008b) found that urinary BPA concentrations in pregnant women in the third trimester were associated with modest elevations (although not statistically significant) in birth weight. Philipat et al. (2012) found that head circumference increased in association with BPA increased concentrations. There is only one cross-sectional pilot study examining the association of urinary BPA concentration with body mass index 
(Wolff et al., 2007). Chou et al. (2011) who measured BPA in maternal blood and umbilical cord blood found elevated prenatal BPA exposure increased the risk of low birth weight, smaller size for gestational age and adverse actions of adipokines in neonates, especially in male infants. Although these results could suggest evidence that maternal exposure may be correlated with adverse birth outcomes, most of these studies are cross-sectional, relied on a single measure of exposure or did not adequately adjust for important potential confounders.

Only one prospective cohort study had examined the relationship of serial BPA urinary concentrations in pregnant women with neurobehavioural outcomes (Braun et al., 2009; 2011). This study found a positive association between urinary BPA concentrations measured during pregnancy and externalizing behaviours (i.e. aggression and hyperactivity). In the follow-up of 3-year-old children, the investigators found gestational BPA exposure to affect behavioural and emotional regulation domains at 3 years of age, especially among girls.

Six cross-sectional analyses of data from the United States National Health and Nutrition Examination Survey (NHANES) reported associations of BPA exposure with self-reported diagnosis of pre-existing cardiovascular disease, hypertension, obesity and diabetes (Lang et al., 2008; Melzer et al., 2010, 2011; Carwile et al., 2011: Silver et al., 2011; Shankar et al., 2012a,b,c). Two other studies in US (Metlzer et al., 2012) and China (Wang et al., 2012) reported an association between BPA exposure and coronary disease at the time of diagnosis and obesity and insuline resistance, respectively. In addition, a study found associations between urine BPA and immune function and allergy (Clayton et al., 2011). These cross-sectional analyses have several important weaknesses that limit their interpretation. A major limitation is the use of a single spot urine sample that reflects recent BPA exposure only (past several $h$ ) and may not adequately measure BPA exposure during the relevant etiological window for cardiovascular disease and diabetes, which might be years or decades earlier.

Strong conclusions based on cross-sectional analyses are not possible. Prospective studies with serial exposures to BPA assessed during etiologically relevant windows, years before development of disease, are needed. Additional studies, especially of a longitudinal design with repeated BPA measurements, are needed to further elucidate these associations.

Casas and co-workers (Casas et al., 2013) pointed out that although there is little published data in mother-child cohorts, many measurements are ongoing (KasperSonnenberg et al., 2012) and they recommend that cohorts start working towards combined and comparison studies. Recommendations for further data collection on BPA include: i) a better evaluation of exposure to BPA in children; ii) repeated measurements of BPA; iii) validation and harmonisation of questionnaires, and iv) detection methods and measurement of BPA. Furthermore, evaluation of mother child cohorts is needed to gain insight in the relation between BPA exposure and effects in children.

Recently, Maserejian and coworkers (2012) investigated whether greater exposure to dental composites is associated with psychosocial problems in children. They performed an analysis of treatment-level data from the New England Children's Amalgam Trial, a 2group randomized safety trial comparing amalgam with the treatment plan of bisphenol A-glycidyl methacrylate (bisGMA)-based composite and urethane dimethacrylate-based polyacid-modified composite (compomer), among 534 children aged 6 to 10 years at baseline. They found that children with higher cumulative exposure to bisGMA-based composite had poorer follow-up scores on 3 of 4 BASC-SR global scales: Emotional Symptoms, Clinical Maladjustment, and Personal Adjustment. Associations were stronger with posterior-occlusal (chewing) surfaces, where degradation of composite was more likely. They concluded that greater exposure to bisGMA-based dental composite restorations as potential source of BPA was associated with impaired psychosocial function in children, whereas no adverse psychosocial outcomes were observed with either urethane dimethacrylate-based compomer or amalgam treatment levels. 


\section{Conclusions}

Conclusions valid for the risk assessment based on cross-sectional analyses are not possible. In addition, many of the available cross-sectional analyses have several important weaknesses that limit their interpretation. The major limitation is the use of a single spot urine sample that reflects recent BPA exposure only (past several hours) and may not adequately measure BPA exposure during the relevant etiological window for cardiovascular disease and diabetes, which might be years or decades earlier. However, also based on the outcome of animal studies, some effects like neurobehavioral outcomes, even when observed in one study, need further investigation for confirmation of negation of the observed effects.

There is a need for further clarification and interpretation of the relationship between BPA exposure and adverse health effects. Prospective studies with serial exposures to BPA assessed during etiologically relevant windows, years before development of disease, and thus prolonged follow up periods are needed.

Specific recommendations for use of existing data include i) the development of conversion models for the different media used for measurement of BPA, ii) and interlaboratory comparisons and calibrations.

Recommendations for further data collection on BPA include: i) a better evaluation of exposure to BPA in children; ii) repeated measurements of BPA; iii) validation and harmonisation of questionnaires; and iv) detection methods. Additionally, evaluation of mother-child cohorts is needed to gain insight in the relation between BPA exposure and effects in children.

A recent overview (Rochester 2013) did identify 91 epidemiological studies showing various associations between adverse effects in humans and environmental BPA exposure, but concluded that these studies were primarily supportive and that a causal relationship was still difficult to obtain (Rochester 2013).

\subsection{Alternatives to BPA currently use}

Some chemicals, similar to BPA, are considered to be able to partially replace BPA in the industrial applications, and, therefore, used in the manufacture of resins and plastics. An example is bisphenol-S [bis(4-hydroxyphenyl)sulfone, (BPS)] whose two phenolic rings are joined together with sulfur. The material containing BPS is of interest in the preparation of high temperature resistant thermosetting thermoplastic polymers (Spitsbergen et al., 1971). Epoxy resin based on BPS has the advantage of resistance to deformation by heat and thermal stability. Such improved epoxy resins have other advantages in briefer gel periods and more rapid development of mechanical properties in cured systems, better resistance to organic solvent attack, increased dimensional stability and better wetting of glass reinforcement. BPS is commonly used as a monomer in the production of epoxy resins (Rwei et al., 2003), cyclic carbonates (Kim et al., 2001), and sulphonated poly(ether ketone ether sulphone) (Changkhamchom and Sirivat, 2010). It is also an important chemical additive in pesticides, dyestuffs, colourfast agents, leather tanning agents, dye dispersants, and fiber improvers.

To date, BPS replaced BPA as a developer in dyes for thermal paper in Japan (Watanabe et al., 2004) and China (Liu, 2005). Therefore, the production and demand for BPS increases year by year. Resultantly, BPS could be a widespread environmental pollutant in future as well as BPA. Even more so, it has been found that BPS is much less biodegradable than BPA (Ike et al., 2006). In this study of eight bisphenol compounds, BPS was the most persistent. Recently, BPS was also detected in canned food (Viñas et al., 2010). Although it has not been studied as much as BPA, preliminary studies show that it shares hormone-mimicking properties as well (Hashimoto et al., 2001; Chen et al., 2002; Kuruto-Niwa et al., 2005; Kitamura et al., 2005). However, studies on the endocrine disrupting properties of BPS have focused on its interaction with human 
estrogen receptor alpha (hERa). Recently, interactions of BPS with other nuclear receptors were also reported (Molina-Molina et al., 2013). Several alternatives for BPA were investigated including BPS, BPF and halogenated BPA derivatives tertrachlorobisphenol A (TCBPA) and tetrabromobisphenol A (TBBPA) for their in vitro interaction with several nuclear receptors (e.g. estrogen receptor a, estrogen receptor $\beta$, androgen receptor, and pregnane $X$ receptor). Although some differences were noted, all nuclear receptors investigated were activated by one or more of the bisphenol alternatives. BPS, BPF and BPA effectively activated both estrogen receptors, whereas BPA, TCBPA and TBBPA were pregnane $X$ receptor agonists. Relative to BPA. the alternatives BPS and BPF and TCBPA and TBBPA showed reduced endocrine activity in the in vitro assays used.

Bisphenol-F, [bis(4-hydroxyphenyl)methane, (BPF)], which has no substituent at the bridging carbon, has a broad range of industrial applications. The BPF monomer is polymerized to prepare the epoxy resins and PC used to manufacture lacquers and varnishes, coatings, adhesives plastics, and other products (Jana et al., 2005). BPF was detected in environmental samples (Fromme et al., 2002; Stachel et al., 2003) and several reports have confirmed its estrogenic effect using various in vivo (Yamasaki et al., 2002) and in vitro assays (Hashimoto and Nakamura, 2000; Hashimoto et al., 2001; Cabaton et al., 2009). Moreover, anti-androgenic activity of BPF has also been observed in several human recombinant cell lines carrying hAR (Satoh et al., 2004; Cabaton et al., 2009).

Like BPA, both BPS and BPF are also an emerging group of environmental contaminants (Fukazawa et al., 2001; de Wit et al., 2010) and interact with and disrupt thyroid hormone receptor signaling (Kitamura et al., 2002). In addition, TBBPA and TCBPA are potent peroxisome proliferator-activated receptor gamma (PPARY) agonists (Riu et al., 2011).

\subsection{Recommendations for research}

\section{General}

For several endpoints like effects on metabolism, neurobehavioral effects, and effects on mammary gland development, the reported studies indicate that BPA exposure may affect these endpoints in animals. However, the overall evidence is equivocal and sometimes based on limited studies. Especially low dose potentially non-monotonic effects are very difficult to identify as such. Therefore, using a weight of evidence approach, as formulated in the conclusions of the various sections, it was concluded that the evidence was not substantiating the suggested adverse health effects. Therefore, recommendations were formulated for further clarification of these issues.

\section{Exposure}

This is the area in which more and better information is needed, regarding the composition and the release of BPA from medical devices in the actual use conditions. For sterilization of medical devices, it is known that steam sterilization may result in release of BPA from PC medical devices. Whether ethylene oxide (EtO) sterilization induces release of BPA from PC or PSU medical devices is yet unknown. Research into the use and consequences of EtO sterilization with regard to BPA release is also recommended.

\section{Hazard}

There are several indications that BPA does have biological effects below the current NOAEL of $5 \mathrm{mg} / \mathrm{kg} \mathrm{b.w./day} \mathrm{and} \mathrm{the} \mathrm{recently} \mathrm{calculated} \mathrm{BMDL}_{10}$ of $8.96 \mathrm{mg} / \mathrm{kg} \mathrm{b.w./day}$ (oral repeated exposure). Because of the uncertainty in the studies focussing on specific outcome parameters it would be advisable to further investigate endpoints like neurotoxicity and neurodevelopmental effects, immunotoxicity, metabolic effects and proliferation of the mammary gland. 
There is a need for further clarification and interpretation of the relationship between BPA exposure and adverse health effects in man. Prospective well conducted epidemiological studies are needed with serial exposures to BPA assessed continually during etiologically relevant windows, years before development of disease, and thus prolonged follow up periods are needed.

Additionally, evaluation of mother child cohorts is needed to gain insight in the relation between BPA exposure and effects in children. It has been suggested (Casas et al., 2013) that for mother-child cohorts, many measurements are already ongoing. 


\section{OPI NION}

\section{Background}

Bisphenol A [Bis(4-hydroxyphenyl)propane], BPA is a high-production volume industrial chemical, being a key building block of polycarbonate (PC) plastic and a precursor for the manufacturing of monomers of epoxy resins. Polycarbonate is used in a wide variety of products including medical devices, for its balance of toughness, dimensional stability optical clarity, high heat resistance and electrical resistance. Currently many scientific discussions are ongoing on the possible adverse effects of BPA. The BPA exposure of the population is via food as a result of the use of BPA in food packaging and via skin as a result of contact with thermal paper. More specifically safety concerns have been expressed for vulnerable groups such as infants, pregnant and breast-feeding women exposed to BPA through other products. Medical devices are a specific product category in which BPA is often found and released during the actual conditions of use. Examples include implants, catheters, tubing and some dental devices fabricated from monomers such as bisphenol $A$ glycidyl methacrylate (Bis-GMA) and very few from bisphenol $A$ dimethacrylate (Bis-DMA) derived from BPA. BPA-resins are also used in inks and adhesives. In addition to BPA itself, polymers produced using BPA like polysulfone (PSU) that are used in medical devices are also considered because they can release BPA. For example, the BPA derived polymer polysulfone (PSU) is used as membrane in hemolysis dialyzers. Some BPA-containing medical devices may have direct and/or indirect contact with the patients (e.g. hemodialyzer apparatus, filters, bypasses, tubing, pumps, instruments, surgical equipment, blood pathway circuits and respiratory tubing circuits). These products are used on all types of patients (e.g. adults, children). Therefore, the SCENIHR was asked for an Opinion regarding the use of BPA in medical devices. This Opinion describes the risk assessment of exposure to BPA via medical devices manufactured from materials potentially leaching BPA, for which the exposure routes are not limited to oral applications (i.e. for dental material) as also exposure via other applications such as subcutaneous and intravenous (e.g. during haemodialysis) routes may occur.

\section{Previous risk assessments}

In the previous evaluations, the following conclusions have been drawn for oral route of exposure to BPA:

- No Observed Adverse Effect Level (NOAEL) of $5 \mathrm{mg} / \mathrm{kg} \mathrm{b.w./day} \mathrm{in} \mathrm{rats} \mathrm{and} \mathrm{mice}$

- Tolerable Daily Intake (TDI) of $50 \mu \mathrm{g} / \mathrm{kg}$ b.w.

- developmental toxic effects only observed at doses with severe maternal toxicity in rats and mice

- an overall NOAEL for reproductive toxicity of $50 \mathrm{mg} / \mathrm{kg} \mathrm{b.w./day} \mathrm{in} \mathrm{rats} \mathrm{and} \mathrm{mice}$

- in terms of toxicokinetics there is a difference between rats and humans (the latter presenting a shorter half-life) as well as between the oral and the parenteral route of exposure.

- due to the first pass effect, after oral uptake, the systemic exposure to free BPA is a small fraction of the external dose in all species.

- there are still unresolved issues in the risk assessment of BPA after oral uptake.

In its recent evaluation EFSA derived a temporary TDI (t-TDI) of $4 \mu \mathrm{g} / \mathrm{kg} \mathrm{b}$.w./day (EFSA 2015). EFSA used for the derivation of the t-TDI a Benchmark dose modelling approach and derived a BMDL 10 value of $8960 \mu \mathrm{g} / \mathrm{kg} \mathrm{b.w./day} \mathrm{based} \mathrm{on} \mathrm{effects} \mathrm{on} \mathrm{the} \mathrm{kidney} \mathrm{in} \mathrm{a}$ multigeneration reproductive toxicity study in mice using oral exposure (Tyl et al., 2008). Similar results (liver and kidney toxicity) were obtained in a rat multigeneration study (Tyl et al., 2002) It should be noted that the internal exposure of the organs is different: hepatic exposure is presystemic, whereas renal exposure is systemic. The doses at the site of action (i.e. liver and kidney) differ after the oral route of exposure because of the biotransformation occurring in the liver/small intestines which results in a lower dose of 
free BPA for kidney exposure. However, this is different for non-oral routes of exposure for which BPA may be directly systemically available. The obtained $B M D L_{10}$ dose, estimated to be the low dose inducing a $10 \%$ deviation compared to control animals, was translated into a human dose inducing similar effects, the human equivalent dose (HED). The HED was determined considering the ratio of internal exposure in mice versus the internal exposure in humans based on toxicokinetic studies. So, the $\mathrm{BMDL}_{10}$ of 8960 $\mu \mathrm{g} / \mathrm{kg}$ b.w./day in mice translates to a HED of $609 \mu \mathrm{g} / \mathrm{kg}$ b.w./day. An uncertainty factor of 25 could be established being based on the following considerations: 1 (as the HED is accounting for interspecies differences in kinetics) $\times 2.5$ (interspecies dynamic factor) $\times 10$ (intraspecies factor) $=25$. An additional uncertainty factor of 6 was applied as a result of the uncertainty analysis related to mammary gland, reproductive, neurobehavioural, immune and metabolic system effects. Therefore, a BPA specific total uncertainty factor of 150 was applied resulting in the t-TDI of $4 \mu \mathrm{g} / \mathrm{kg} \mathrm{b.w./day.} \mathrm{In} \mathrm{view} \mathrm{of} \mathrm{the} \mathrm{still} \mathrm{present}$ uncertainties the TDI was declared temporary.

The SCENIHR considered that the recently derived temporary TDI ( $t$-TDI) of $4 \mu \mathrm{g} / \mathrm{kg}$ b.w./day by EFSA which takes effects and uncertainties into account, as well the use of a BPA specific uncertainty factor of 150 , represents a useful base for carrying out a BPA risk assessment for the use of BPA in medical devices.

The main focus of the previous evaluations was on the oral route of exposure. In its latest evaluation EFSA also included the dermal route of exposure (EFSA 2015). Especially for medical devices manufactured from PC plastics, other exposure routes such as subcutaneous and intravenous (e.g. during heamodialysis) are important.

\section{General exposure}

The human population is exposed to BPA through the diet, while air, dust, water and skin contact primarily through thermal paper are other sources of exposure. BPA in food and beverages accounts for about $50 \%$ of daily human internal exposure whereas skin contact with BPA-containing thermal paper is the source for the another $50 \%$ of internal expsoure. BPA exposure results from either the release of non-polymerized monomers or from the slow decay of polymer bonds in PC leading to monomer release into proximal foods and liquids. A number of studies in various countries have indicated that the vast majority of the population ( $91-99 \%$ ) does have detectable levels of BPA-conjugates in urine. The measured BPA levels reflect the recent exposure of the past several hours before the sample collection as there is a rapid conjugation and short elimination halftime of a few hours of BPA in blood.

Notably, regarding BPA determination, the analytical method used to detect both the parent compound and its metabolites is crucial especially at the low levels expected in biological samples. The sampling and analytical methods used, therefore, can represent a relevant source of differences among available studies. In addition, as BPA has a widespread use (e.g. blood sampling devices) contamination of samples, which is particularly relevant for plasma samples, can occur leading to erroneously elevated concentrations. In urine, BPA is present in its conjugated form. Urinary biomonitoring data provide information on the internal dose, which is the result of total BPA exposure, independently from the sources. Therefore, biomonitoring data in urine account not only for dietary exposure, but also for non-food sources (e.g. medical devices, dust, thermal and other kind of papers). Since BPA urinary excretion is almost complete within 24 hours after exposure and due to the less invasively sampling, urine is the matrix of choice for assessing daily exposure to BPA in humans. Blood and urine concentrations of total BPA (free plus conjugates) determined at one time point are not representative of an average exposure, because the results are strongly dependent on the time of blood sampling with respect to the exposure time.

On the basis of available biomonitoring and exposure data, it was until now concluded that the exposure to BPA from non-food sources is generally lower than that from exposure from food by at least one order of magnitude for most studied subgroups. Dietary exposure was estimated to contribute for more than $90 \%$ to the overall BPA- 
exposure for non-occupationally exposed individuals (Geens et al., 2012; EFSA, 2013; ANSES, 2013) and exposure through dust ingestion, dental surgery and dermal absorption from thermal paper accounted for less than $5 \%$. However, the contribution due to medical devices has never been taken into account.

More recently EFSA (2015) estimated the dietary exposure to be highest for infants and toddlers among the population older than 6 months and up to 10 years of age, with an estimated average of $375 \mathrm{ng} / \mathrm{kg}$ b.w./day and a highest estimated dietary exposure of $857 \mathrm{ng} / \mathrm{kg}$ b.w./day. For breastfed infants days 1 - 5 after birth $435 \mathrm{ng} / \mathrm{kg} \mathrm{b.w./day} \mathrm{was}$ estimated as high dietary exposure. The modeled dietary exposure for teenagers, adults and the elderly ranged from 116 to $159 \mathrm{ng} / \mathrm{kg}$ body weight per day for average exposure, with a high exposure ranging from 341 to $388 \mathrm{ng} / \mathrm{kg}$ body weight per day. The non-dietary exposure to thermal paper, when expressed as oral dose equivalent, was estimated for adolescents and adults to be two to three times higher than the oral exposure (EFSA, 2015).

\section{Skin absorption}

The available data indicate that at the estimated exposures, BPA penetrated into the skin by passive transfer at a percentage between $10 \%$ and up to $47 \%$ of the applied dose. The possible skin metabolism is controversial, since contrasting results were reported; therefore, excluding a pre-systemic BPA clearance due to dermal biotranformation, as a worst-case a systemic bioavailability in the range of $10-30 \%$ of the applied dermal dose can be used for risk assessment purposes. EFSA has used a value of $10 \%$ in its risk assessment (EFSA 2015), assuming that the amount present in the stratum corneum will remain deposited on the skin surface, for reasons emerging from the PBPK modelling.

\section{$\underline{\mathrm{SC} \text { exposure/administration }}$}

Subcutaneous administration resulted in much higher free BPA levels compared to the oral administration. However, differences in circulating free BPA after subcutaneous and oral administration can disappear within two hours as demonstrated in a study in rats.

\section{IV administration}

After intravenous administration a rapid distribution to organs and rapid clearance from blood was described. Initial high serum levels of free BPA may partly distribute and sequester into fatty tissues. BPA was eliminated from adipose tissue at a rate similar to that for BPA conjugates in the whole organism indicating the non-persistent nature of BPA. Free BPA was no longer detectable in serum of mice at $8 \mathrm{~h}$ after i.v. administration.

\section{Inhalation}

No information was available for inhalation exposure that might occur after intubation for inhalation support in intensive care units. However, in vitro studies showed a lack of conjugation of BPA by lung cells.

For risk assessment purposes, the bioavailability of free BPA is crucial as this is the active compound. However, data on both free and conjugated BPA are required to assess the exposure and fate of BPA.

\section{Exposure from medical devices}

Medical devices based on PC and polysulfone, due to their chemistry, can contain BPA residues whereas others like PVC may or may not contain BPA residues depending on their production method. In addition, some other BPA-derivatives (such as epoxy resins) are used specifically in dental materials. The major factor influencing the residual amount of BPA levels is the employment of incorrect operating conditions during the processing step. Moreover, breakdown or hydrolysis of the PC polymer after manufacturing can occur, thus giving rise to the free monomer from the polymer available for exposure. In PC articles used for food contact, the residual content is usually less than $10 \mu \mathrm{g} / \mathrm{g}$ of PC (ECB, 2003). 
Exposure can be estimated by either measuring the BPA content of the medical devices or by extraction assays for potential release. Extraction of BPA was much more prominent in aqueous ethanol ( $17.2 \% \mathrm{v} / \mathrm{v})$ and bovine serum (mimicking human serum) than in water. For PC casings of hemodialyzers and hollow fibers used in hemodialyzers extracted amounts of BPA were ranging from $0.2-12.2 \mathrm{mg} / \mathrm{kg}$. Under simulated use conditions release in bovine serum was up to $2090 \mathrm{ng} /$ dialyzer, and in aqueous ethanol $(17.2 \% \mathrm{v} / \mathrm{v})$ up to $4299 \mathrm{ng} /$ dialyzer. For dental materials the leakage is limited to resins composed of Bis-DMA (Bisphenol A dimethacrylate) with an ester linkage that can be hydrolysed to BPA, whereas the ether linkage in Bis-GMA (Bisphenol A glycidyl methacrylate) was found to be stable. Dental materials release BPA especially shortly (01 hour) after placement, the levels of BPA detected in saliva ranged from $0.64 \mathrm{ng} / \mathrm{mL}$ to $30 \mu \mathrm{g} / \mathrm{mL}$. This wide range in BPA measurements may indicate a continuous reduced leaching of BPA from dental materials, as well as reflect variations in the analytical methodology used.

Little information concerning BPA exposure resulting from the use of medical devices is available. For the placing of dental composite resin restorations, measurements have shown the release of BPA mainly during the few hours directly after application. Values measured were up to $30 \mu \mathrm{g} / \mathrm{mL}$ saliva, and $931 \mu \mathrm{g}$ in total saliva volume produced. Calculations based on the maximum values of BPA found in fissure sealants and in composite materials, in combinations with the actual amount of material used in clinical practice and a median 4-year life-time of a composite restoration, suggest a maximum exposure of $0.06 \mu \mathrm{g} \mathrm{BPA} /$ day from fissure sealants, and a maximum exposure of $0.36 \mu \mathrm{g}$ $\mathrm{BPA} /$ day from composite restorations. Contact with dental materials gave an estimated short term $(<24 \mathrm{~h}$ ) exposure of 140 to $200 \mathrm{ng} / \mathrm{kg}$ body weight per day for childrenand adults, respectively. These BPA releases contribute to the oral exposure to BPA and are included in the biomonitoring studies to the total exposure to BPA as mentioned above. In general, there was very limited information provided to assess the reliability of available data.

Taking into account the many possible sources of exposure of patients during hospital care the material used, information related to BPA leaching, the duration of a single treatment and the frequency of treatments, several exposure scenarios were evaluated giving rise to toxicologically relevant acute, short- and long-term exposure. Due to the scarcity of information related to release of BPA from medical devices, six critical exposure scenarios were evaluated to estimate potential exposure to BPA from medical devices (Table 6). The highest exposures estimated occurred during exposure of prematurely born infants in NICU (3000 ng/ $\mathrm{kg}$ body weight per day) and prolonged medical procedures in infants such as extracorporeal circulation (by-pass) or transplantation/ implantation of an artificial organ $(685 \mathrm{ng} / \mathrm{kg}$ body weight per day, b.w. around 5 kg.b.w.).

Measurements in dialysis patients found BPA values up to $6.6 \mathrm{ng} / \mathrm{mL}$ blood. In prematurely born infants undergoing intensive therapeutic medical interventions for BPA geometric mean urinary concentration of $30.3 \mu \mathrm{g} / \mathrm{L}$ was observed with the highest value measured $946 \mu \mathrm{g} / \mathrm{L}$, which was about ten times higher than that among children 6-11 years old. More than $90 \%$ of the BPA detected in the urine of the prematurely born infants was in its conjugated (e.g. glucuronide, sulfate) form.

The use of medical devices consisting of BPA containing PVC for treatment of adults (1000 ng/kg b.w./day), and treatment of prematurely born infants ( $7000 \mathrm{ng} / \mathrm{kg}$ body weight per day, $7 \mu \mathrm{g} / \mathrm{kg}$ body weight per day) also results in potential high exposures. Short term exposure via medical devices consisting of BPA containing PVC might even be higher (adults up to $5000 \mathrm{ng} / \mathrm{kg}$ b.w./day, infants up to $12000 \mathrm{ng} / \mathrm{kg} \mathrm{b.w./day).}$ However, it is worth noting that exposure to BPA via BPA-containing PVC has been estimated based on extrapolation from data on phthalate leakage from PVC and are, therefore, affected by a high degree of uncertainties. In addition European PVC manufacturers informed that they do not use BPA in their PVC production, resulting in 
expected low level of exposure. However, medical devices from Third Countries can be marketed; these includes gloves, on which no information is available.

Some of the estimated BPA exposures due to medical devices are in the same range as exposure via the food (EFSA, 2015). EFSA estimated the dietary exposure to be highest for infants and toddlers among the population older than 6 months, with the estimated average of $375 \mathrm{ng} / \mathrm{kg} \mathrm{b.w./day} \mathrm{and} \mathrm{a} \mathrm{highest} \mathrm{estimated} \mathrm{dietary} \mathrm{exposure} \mathrm{of} 857 \mathrm{ng} / \mathrm{kg}$ b.w./day. The modeled dietary exposure for teenagers, adults and the elderly ranged from 116 to $159 \mathrm{ng} / \mathrm{kg}$ body weight per day for average exposure, with a high exposure ranging from 335to $388 \mathrm{ng} / \mathrm{kg}$ body weight per day. The non-dietary exposure to thermal paper, when expressed as oral dose equivalent, is for adolescents and adults two to three times higher than the oral exposure (EFSA, 2015). However, the bioavailability of BPA through routes of exposure other than the oral (such as the one associated to medical devices use) is much higher.

Table 6: BPA exposure from medical devices as estimated for various use scenarios.

\begin{tabular}{|c|c|c|c|c|}
\hline \multirow[b]{2}{*}{ Exposure scenario } & \multicolumn{4}{|c|}{ BPA exposure estimation in $\mathrm{ng} / \mathrm{kg}$ b.w./ day } \\
\hline & $\begin{array}{c}\text { Prematurely } \\
\text { born infant }\end{array}$ & Infant & Child & Adult \\
\hline $\begin{array}{l}\text { External contact with a MD containing BPA } \\
\text { (short-term) }\end{array}$ & 1 & & & 0.08 \\
\hline $\begin{array}{l}\text { Contact with dental material } \\
\text { (Acute exposure }<24 \mathrm{~h} \text { ) } \\
\text { (long-term) } \\
\text { Contact with orthodontic equipment } \\
\text { (Acute exposure }<24 \mathrm{~h} \text { ) } \\
\text { (short-term) } \\
\text { (long-term) }\end{array}$ & $\begin{array}{l}\text { na } \\
\text { na }\end{array}$ & $\begin{array}{l}\text { na } \\
\text { na }\end{array}$ & $\begin{array}{c}2 \\
140 \\
13.5 \\
12\end{array}$ & $\begin{array}{c}200 \\
6 \\
140 \\
7.5 \\
6\end{array}$ \\
\hline $\begin{array}{l}\text { Contact with an implant } \\
\text { (short-term) } \\
\text { (long-term) }\end{array}$ & & & $\begin{array}{l}11 \\
0.8\end{array}$ & $\begin{array}{c}6 \\
0.4\end{array}$ \\
\hline Hemodialysis (long-term) & & & & 57 \\
\hline $\begin{array}{l}\text { Prolonged surgical procedures } \\
\text { (short-term) }\end{array}$ & & 685 & 114 & 57 \\
\hline $\begin{array}{l}\text { Prolonged exposure to different sources of } \\
\text { BPA in intensive care units } \\
\text { (short-term) }\end{array}$ & 3000 & & & \\
\hline $\begin{array}{l}\text { Breast pump and collection vessel made of } \\
\text { PC } \\
\text { (short-term) }\end{array}$ & & 134 & & \\
\hline $\begin{array}{l}\text { Uses of PVC } \\
\text { (short-term) } \\
\text { (long-term) }\end{array}$ & $\begin{array}{c}12000 \\
7000\end{array}$ & & & $\begin{array}{l}5000 \\
1000\end{array}$ \\
\hline
\end{tabular}




\section{BPA metabolism and toxicokinetics in humans}

The unchanged parent BPA is the toxic species, which is readily detoxified in the body. The major BPA metabolite in human is BPA-glucuronide, which is quantified in plasma and rapidly excreted in the urine; BPA-sulphate was also detected after oral exposure as a minor urinary metabolite. After oral exposure there is a very fast first pass effect in the liver that results in very small amounts of unchanged parent BPA, up to $9.5 \%$ of the oral administered dose is recovered as non-conjugated BPA in human urine. In humans, a polymorphism exists for the conjugation of BPA. However, the polymorphism was found to result in a limited level of variability of BPA conjugation as indicated by biomonitoring studies. Therefore, it can be considered that the default value used to account for kinetic interindividual variability within the general population (IPCS, 2006), can cover differences due to polymorphically expressed enzyme activity involved in BPA metabolism.

In humans, both low and high single oral doses of BPA are well absorbed ( $>90 \%$ ). This is a conservative estimate for neonates. The half-life of BPA in humans is very short, ranging from 1 to $3.5 \mathrm{~h}$, and because of high first-pass metabolism in the liver the systemic availability is expected to be low. Based on the analysis of oral versus intravenous toxicokinetic data, the oral systemic bioavailability of unconjugated BPA is $2.8 \%, 0.2 \%, 0.9 \%$ and less than $1 \%$ in rats, mice, monkeys, and dogs respectively. The systemic availability of unconjugated BPA in humans has not been evaluated experimentally, however, controlled biomonitoring studies indicated that internal exposure in humans to unconjugated BPA is very low (1 - 10\%).

Studies on toxicokinetics of BPA available to date in animals have demonstrated a significantly lower internal exposure to free BPA after oral intake as compared to parenteral exposure. This is essentially due to the highly efficient pre-systemic conjugation to glucuronides and sulfate which occurs mainly in the liver and partially in the gut after oral administration independently of the species. Thus, the internal exposure to free BPA after oral intake is lower as compared to dermal or parenteral exposure, although also for these latter routes of exposure the metabolization in the liver quickly diminishes free circulationg free BPA.

After dermal exposure, the absorption fraction can be considered in the range of $10-30$ $\%$ of the applied dose as a worst-case assumption, which is directly systemically bioavailable. EFSA has used a value of $10 \%$ in its risk assessment (EFSA, 2015) assuming that the amount present in the stratum corneum will remain deposited on the skin surface, for reasons emerging from the PBPK modelling.

For all the parenteral routes of exposure (including i.v., i.p., or subcutaneous) the chemical is $100 \%$ systemically bioavailable: however, the clearance of free BPA from the circulation appeared to be quite fast, as indicated by controlled studies in non human primates showing a half-life in blood of $0.66 \mathrm{~h}$ with $>50 \%$ of circulating BPA already conjugated 5 min after i.v. injection.

The available modeled data, obtained after oral exposure, also point out that newborns and babies up to 6 months constitute a potentially susceptible subpopulation due to immature BPA metabolism but that the default factor which is used to account for the toxicokinetic variability in the general population seems to be large enough to cover the variability in the newborn population exposed via the oral route. Analogously, interindividual differences in the expression of the isoenzyme mainly responsible for BPA glucuronidation are within a factor of 4 , again covered by the usual the default factor, at the estimated dietary exposures.

\section{Pharmacokinetics in animals}

There are major differences in BPA metabolism and disposition between rodents (enterohepatic recirculation and extensive fecal excretion of unconjugated BPA) and primates (extensive urinary excretion of conjugated BPA), that directly affects BPA halflife. Indeed, rodents (mice and rats) show a prolonged clearance of BPA due to the 
existence of the so-called entero-hepatic recirculation. After uptake from the GI-tract in rats there is a high degree of conjugation of BPA in the liver. However, BPA is excreted from the liver via bile into the GI-tract where it can be cleaved again resulting in free BPA that can be recirculated or excreted via the feces. So, there may be a higher exposure to free BPA especially in neonatal mice and rats after a specific oral dose when compared to humans. In addition, there is another major interspecies difference related to neonatal development in the Phase II metabolism of BPA, which is strongly age-dependent in rodents. Thus, there may be a higher exposure to free BPA especially in neonatal mice and rats after a specific oral dose when compared to humans. Indeed in primates the degree of conjugation was not affected by developmental age, and consequently there was no significant age-related change in internal exposure metrics for free BPA in primates. It may be considered that for neonatal effects, studies in mice and rats may over-predict adverse outcomes in humans (Shelnutt et al., 2013). However, careful consideration is needed in BPA measurments regarding possible unintentional exposure in animal experiments and serum sample contamination during collection and storage (Churchwell et al., 2014).

\section{Toxicity of BPA}

Several repeated dose toxicity studies have been performed in mainly rodents. BPA was found to be of low acute toxicity, and the lowest NOAEL for subchronic oral exposure currently available is approximately $5 \mathrm{mg} / \mathrm{kg} \mathrm{b.w./day,} \mathrm{based} \mathrm{on} \mathrm{effects} \mathrm{on} \mathrm{the} \mathrm{liver} \mathrm{and}$ kidney as target organs. Based on effects on the relative kidney weight in a two generation reproductive toxicity study in mice EFSA has calculated a $\mathrm{BMDL}_{10}$ of 8.96 $\mathrm{mg} / \mathrm{kg} \mathrm{b.w./day} \mathrm{(EFSA,} \mathrm{2015).}$

The $\mathrm{BMDL}_{10}$ represents the lower level of the confidence interval of the effect resulting in a $10 \%$ deviation from vehicle treated control animals. The critical endpoint for this $\mathrm{BMDL}_{10}$ was alteration in relative kidney weight. The two Points of Departure (PoD), i.e. dose for liver toxicity and dose for kidney toxicity, are quantitatively very similar, although conceptually different from a toxicokinetic point of view. Indeed, the internal exposure of the organs is different: the hepatic exposure is presystemic, whereas the renal exposure is systemic.The doses at the site of action (i.e. liver and kidney) differ after the oral route of exposure because of the biotransformation occurring in the liver resulting in a lower dose of free BPA for kidney exposure. The SCHENIR supports the use of the newly calculated t-TDI for the risk assessment of medical devices.

BPA is not a mutagen in in vitro test systems, nor does it induce cell transformation. BPA was shown to affect chromosomal structure in dividing cells in in vitro studies, but evidence for this effect in in vivo studies is inconsistent and inconclusive. In addition, BPA was found to be genotoxic in in vitro micronucleus assay. These findings were not confirmed by in vivo studies. Therefore, BPA is not likely to pose a genotoxic hazard to humans.

In standard carcinogenic testing protocols according to OECD BPA has no carcinogenic activity. In addition, in multigeneration studies no indication of increased cancerogenicity was observed. Studies using subcutaneous administration of BPA indicated that BPA may have the ability to increase the effects of well-known carcinogens even at very low BPA levels. However, the studies had limitations that render them unsuitable to assess whether BPA has a carcinogenic potential (as initiator or promoter) by prenatal or perinatal exposure.

Prenatal exposure to BPA by subcutaneous injection and oral administration at doses between $2.5-1000 \mu \mathrm{g} / \mathrm{kg}$ b.w. induced mammary gland alterations including cell proliferation, some described as pre-neoplastic and neoplastic lesions, in the offspring. Results observed in rhesus monkey also indicated alterations of glandular tissue in the mammary gland. Also, in a recently published animal study there were indications for mammary gland ductal proliferation (Declos et al., 2014).

In summary, at present there are no indications for carcinogenic effects of BPA in OECD guideline studies, but some proliferative effects in the mammary gland have been 
reported. The observed effects on mammary gland development do need further investigation as the biological significance of such alterations as well as the relevance for humans are at the moment not known.

Studies on anxiety (rodent and non-human primate) have a behavioural end-point that is highly depending on study design, including testing apparatus, inclusion of only one sex, age at examination. There is uncertainty with regard to the interpretation of the data. Recent data confirm previous data on the sex-dimorphic effects of BPA on social behaviour. However, it is uncertain whether elimination of sexual dimorphism could be considered adverse for humans. Other effects described in the recent studies may indicate that the effects observed with BPA on hypothalamic organization involves mechanisms different from its estrogenic action because they are very different from those of oestradiol which was used as positive control. This effect was included in the uncertainty analysis by EFSA (2015).

BPA is able to elicit skin sensitization in humans, because it is a weak sensitizer. Studies on a possible relationship between prenatal and/or postnatal BPA exposure and allergic responses are not consistent. Although effects on the immune system are suggested the data are insufficient to draw final conclusions on the immunotoxicity of BPA.In view of these suggested effects of BPA on the immune system further investigation to determine potential immunotoxicity of BPA is warranted. This effect was included in the uncertainty analysis by EFSA (2015).

The toxicological data do not indicate a clear effect of BPA on cardiovascular function.

Several published studies in laboratory animals have directly addressed the issue of whether developmental exposure to BPA can affect adiposity, glucose or insulin regulation, lipid profiles or other end-points related to diabetes or metabolic syndrome. Animal studies, however, have shown contrasting results (e.g. increase, decrease and no effect on body weight). The discrepancy among the various animal studies may arise from variation in experimental conditions, such as dosing regimen, animal species and strains, and timing of evaluation of effects. A number of studies in prenatally- and postnatally exposed rats and mice suggest that BPA exposure has an effect on metabolic function. In some of the studies, the findings have been claimed as evidence of a nonmonotonic dose-response as effects were seen at a lower dose which was not observed in higher doses. However, effects were only seen at one dose level. There are no studies which demonstrate effects of different effect size at two dose levels and no or a reduced effect at a higher dose thus corroborating the existence of non-monotonicity. There is, however, no convincing evidence that BPA is obesogenic later in life after intrauterine exposure or in longer-term studies. Therefore, regarding a metabolic effect of BPA. No clear conclusions can be drawn at the moment due to a lack of consistent evidence. Inconsistent results were also obtained in epidemiological studies. Therefore, this issue still warrants further investigation. This effect was included in the uncertainty analysis by EFSA (2015).

A large number of studies is available on the effects of BPA on reproduction and prenatal development some of which performed according to internationally agreed guidelines and in compliance with GLP principles. A wealth of in vitro results and studies on non-intact animals (such as ovariectomized rodents) is available, but their value for risk assessment is questionable. There are also uncertainties as to reproducibility of several individual studies. These studies were conducted in rats and mice. Overall, female reproductive toxicity after oral exposure occurred with an overall NOAEL of $50 \mathrm{mg} / \mathrm{kg} \mathrm{b.w./day} \mathrm{and} \mathrm{a}$ LOAEL of $500 \mathrm{mg} / \mathrm{kg} \mathrm{b.w./day,} \mathrm{derived} \mathrm{from} \mathrm{the} \mathrm{Tyl} \mathrm{et} \mathrm{al.} \mathrm{(2002)} \mathrm{multigeneration} \mathrm{study.}$ However, at the LOAEL for female reproductive effects, significant body weight reduction and hepatic/renal toxicity occurred. As to developmental toxicity, Bisphenol A does not cause malformations or birth defects in rats or mice at levels up to the highest doses evaluated: $640 \mathrm{mg} / \mathrm{kg}$ b.w./day (rats) and $1250 \mathrm{mg} / \mathrm{kg}$ b.w./day (mice). BPA does not alter male or female fertility after gestational exposure up to doses of $500 \mathrm{mg} / \mathrm{kg}$ b.w./day in the rat (Tyl et al., 2002) and $600 \mathrm{mg} / \mathrm{kg} \mathrm{b.w./day} \mathrm{in} \mathrm{the} \mathrm{mouse} \mathrm{(Tyl} \mathrm{et} \mathrm{al.,}$ 2008, highest dose levels evaluated). BPA does not permanently affect prostate weight at 
doses up to $475 \mathrm{mg} / \mathrm{kg} \mathrm{b.w./day} \mathrm{in} \mathrm{adult} \mathrm{rats} \mathrm{or} 600 \mathrm{mg} / \mathrm{kg}$ b.w./day in mice. BPA does change the age of puberty in male or female rats at high doses (ca. $500 \mathrm{mg} / \mathrm{kg}$ b.w./day). Neurodevelopmental toxicity was not observed at the highest dose tested $(164 / 410 \mathrm{mg} / \mathrm{kg}$ b.w./day, Stump et al., 2010). In a recent study by Delclos et al., (2014) low dose effects for doses below the current NOAEL of 5,000 $\mu \mathrm{g} / \mathrm{kg}$ b.w./day, associated with reproductive parameters and/or estrogenic activity, could not be detected.

On the basis of the above studies, it can be concluded that BPA is not a specific reproductive or developmental toxicant: general toxicity effects such as body and organ weights and liver histopathology and nephropathy often occur simultaneously with reproductive or developmental effects, which are only observed at high dosages. On this basis it was classified as a Repro 2 toxicant (being classification a hazard-based process). Recently, the Committee for Risk Assessment (RAC) of the European Chemicals Agency (ECHA) has classified BPA as a category $1 \mathrm{~B}$ reproductive toxicant in view of the adverse effects on sexual function and fertility in line with a proposal from the French competent authority (ECHA, 2014).

A number of studies in prenatally and postnatally exposed rats and mice indicate that BPA exposure has an effect on metabolic function as evidenced by effects on glucose or insulin in regulation or lipogenesis, and may affect bodyweight gain at least in short-term studies. There is, however, no convincing evidence that BPA is obesogenic later in life after intrauterine exposure or in longer-term studies. In some of the studies, the findings have been considered as evidencing a non-monotonic dose-response as effects were seen at a lower dose which was not observed in higher doses. However, again effects were seen only at one dose level. There are no studies which demonstrate effects of different effect size at two dose levels and no or a reduced effect at a higher dose thus corroborating the existence of non-monotonicity. Additionally, epidemiological studies do not show unequivocal convincing evidence for metabolic effects of BPA.

A limited, but increasing number of epidemiological studies investigated an association between BPA exposure and health outcomes, including altered behavior after dental BPA exposure. Most of them use cross-sectional designs not suitable for identifying a causeeffect relationship, limiting their interpretability, especially for outcomes that have long latency periods (e.g. cardiovascular disease, diabetes). In addition, many of these crosssectional analyses have several important weaknesses that limit their interpretation. The major limitation is the use of a single spot urine sample that reflects recent BPA exposure only (past several hours) and may not adequately measure BPA exposure during the relevant etiological window for cardiovascular disease and diabetes, which might be years or decades earlier. Conclusions based on cross-sectional analyses are not possible.

For further clarification and interpretation of the relationship between BPA exposure and adverse health effect additional studies are needed. These should include prospective studies with serial exposures to BPA assessed during etiologically relevant windows, years before development of disease, and thus prolonged follow up periods. Specific recommendations for use of existing data include i) the development of conversion models for the different media used for measurement, ii) inter-laboratory comparisons and calibrations. Recommendations for further data collection on BPA include: i) a better evaluation of exposure especially in children; ii) repeated measurements over time; iii) validation and harmonization of questionnaires; and iv) adequate detection methods. It has been suggested (Casas et al., 2013) that although there is little published data in mother-child cohorts, many measurements are ongoing which should start working towards combined and comparison studies

\section{Conclusions}

Regarding potential health effects of BPA exposure and the level of exposure, several controversial issues remain, regarding the possibility that BPA does have biological effects below the calculated $\mathrm{BMDL}_{10}$ of $8.96 \mathrm{mg} / \mathrm{kg}$ b.w./day (oral repeated exposure) such as prenatal BPA exposure and an effect on mammary gland development and 
altered behaviour/anxiety, in addition to possible effects on metabolism and adiposity. However, the evidence has been mainly obtained in dedicated studies focussing on specific outcome parameters like adiposity and hormone levels, and not from general toxicity studies. Some of those parameters resulted in contradicting results in various studies (e.g. decrease, no effect or increase in body weight). In addition, dose-response relationships could not be established. In a recent report from studies in rats including both a low dose range and two high doses, toxicity (including effects on the mammary gland) was only observed in the high dose groups whereas doses below the NOAEL of 5 $\mathrm{mg} / \mathrm{kg}$ b.w./day (oral exposure) did not induce toxicity (Delclos et al., 2014). So far, epidemiological studies performed do not provide consistent outcomes to conclude on the relevance of animal findings and on possible human health effects.

For medical devices, several exposure scenarios were identified taking into account the material used, information related to BPA leaching, the duration of a single treatment and the frequency of treatments. They included external short-term contact with a medical device, short and long term contact with dental materials, short and long-term contact with an implanted medical device, long-term contact via hemodialyzers and short-term contact in intensive care units with various medical devices. They were evaluated in view of toxicologically relevant acute, short- and long-term exposure.

For BPA exposure resulting from the use of medical devices, little information is available and in many cases due to the lack of experimental data, estimates were used. Some of the estimated BPA exposures due to medical devices are in the same range as exposure to BPA via food (EFSA 2013). The comparison between the level of exposure should consider that, depending on the type of medical device, when the exposure route is parenteral, BPA may be $100 \%$ systemically bioavailable, while the bioavailability of free BPA after oral exposure is considered $1 \%$ of the ingested dose. In addition, with the exception of haemodialysis practice, exposure due to medical devices generally occurs for a limited period of time. However, the uncertainties related to the exposure assessment represent indeed the weakest part of this evaluation. For this reason, only the highest value obtained in the estimate was indicatively used.

The highest exposures estimated occurred during 1) $3000 \mathrm{ng} / \mathrm{kg} \mathrm{b.w./day} \mathrm{(3 \mu g/kg}$ b.w./day) for prematurely born infants in neonatal intensive care units; 2) $685 \mathrm{ng} / \mathrm{kg}$ b.w./day for prolonged medical procedures (such as extracorporeal circulation or transplantation/ implantation of an artificial organ) in infants (b.w. around $5 \mathrm{~kg}$ ); 3) 57 $\mathrm{ng} / \mathrm{kg}$ b.w./day for dialysis patients; 4) $0.4-12 \mathrm{ng} / \mathrm{kg} \mathrm{b.w./day} \mathrm{for} \mathrm{long} \mathrm{term} \mathrm{exposures}$ to medical devices; 5) 140 to $200 \mathrm{ng} / \mathrm{kg} \mathrm{b.w./day} \mathrm{for,} \mathrm{respectively,} \mathrm{children} \mathrm{and} \mathrm{adults}$ due to contact with dental materials $(<24 \mathrm{~h}) ; 6) 2-12 \mathrm{ng} / \mathrm{kg}$ b.w./day due to long term contact with dental materials, far below the exposure to BPA via food (EFSA 2013, EFSA 2015).

The exposure of neonates is about 13-fold higher than the mean dietary exposure of breastfed infants at days 1 - 5 after birth ( 7 fold higher than the high exposure for infants day 1 - 5 after birth $435 \mathrm{ng} / \mathrm{kg}$ b.w/day), whereas the external dose related to infants repeated treatmens with medical devices was in the same range (high exposure for population older than 6 months $857 \mathrm{ng} / \mathrm{kg}$ b.w./day) (EFSA 2013, EFSA 2015). However, considering the different bioavailability of the two routes (oral vs parenteral) the internal exposure due to medical devices is $2-3$ order of magnitude higher.

The use of medical devices consisting of BPA containing PVC during treatment of adults (1000 ng/kg b.w./day) and prematurely born infants (7000 ng/kg b.w./day) as single use or after short-term medical device use (adults up to $5000 \mathrm{ng} / \mathrm{kg} \mathrm{b.w./day,} \mathrm{infants} \mathrm{up} \mathrm{to}$ $12,000 \mathrm{ng} / \mathrm{kg}$ b.w./day) might even be higher. However, a serious limitation of these data is that the levels of exposure due to BPA containing PVC use was estimated by extrapolating the migration properties of DEHP, as no data on BPA leakage were available. In addition European PVC manufacturers informed the SCENIHR that they do no longer use BPA in PVC production. However, medical devices from Third Countries can be marketed; these include gloves, on which no information is available. 
From the available data, concerning exposure via the oral route it can be concluded that the oral long-term exposure via dental material is far below the current oral t-TDI of $4 \mu \mathrm{g} / \mathrm{kg} \mathrm{b.w./day} \mathrm{and} \mathrm{poses} \mathrm{negligible} \mathrm{risk} \mathrm{for} \mathrm{human} \mathrm{health.}$

Regarding the parenteral exposure via medical devices, it is necessary to consider the differences in bioavailability (assumed as $100 \%$ ) when compared to the oral route (around $1 \%$ ). Therefore, the SCENIHR considered it appropriate to make the comparison using the estimated internal dose $(6 \mu \mathrm{g} / \mathrm{kg}$ b.w./day $=\mathrm{HED} / 100)$ rather than the external t-TDI.

Taking treatment of neonates in intensive care units as an example, exposure of $3 \mu \mathrm{g} / \mathrm{kg}$ b.w./day (assuming as $100 \%$ systemically bioavailable) is, before adjustment for route specific systemic availability, below the oral t-TDI of $4 \mu \mathrm{g} / \mathrm{kg}$ b.w./day. However, it is about 75-fold higher when compared to the internal exposure to free BPA using the oral t-TDI (being $0.04 \mu \mathrm{g} / \mathrm{kg}$ b.w./day based on a t-TDI of $4 \mu \mathrm{g} / \mathrm{kg}$ b.w./day with $1 \%$ systemic bioavailability for the oral dose). Alternatively, applying a MOS approach, when the internal HED of $6 \mu \mathrm{g} / \mathrm{kg} \mathrm{b.w./day} \mathrm{was} \mathrm{compared} \mathrm{to} \mathrm{the} \mathrm{internal} \mathrm{exposure} \mathrm{via} \mathrm{medical}$ devices ( $3 \mu \mathrm{g} / \mathrm{kg}$ b.w./day) a MOS= 2 was obtained, much lower than the assessment factor of 150 used for the t-TDI derivation.

For the other scenarios the MOS is 10 for repeated medical procedures in infants; for the other short term exposure scenarios estimated for different medical devices, the MOS ranges between 43 and 100 ; or dialysis treatments the MOS is 105 . For the other scenarios the exposure ranges from $0.4 \mathrm{ng} / \mathrm{kg} \mathrm{b.w./day} \mathrm{to} 12 \mathrm{ng} / \mathrm{kg} \mathrm{b.w./day} \mathrm{resulting} \mathrm{in}$ a MOS range from 500 to 15,000 .

In relation to exposure to BPA released from dental materials during the first 24 hours after placement, uptake in the oral cavity by oral mucosa can be considerable. Assuming it $100 \%$ as a worst case scenario, for the highest exposures $200 \mathrm{ng} / \mathrm{kg} \mathrm{b.w./day} \mathrm{the}$ MOS would be 30 . However, the assumption of $100 \%$ bioavailability is largely exaggerated and the peak of exposure occurs for $<24 \mathrm{~h}$ (representative of acute exposure in a toxicological context) whereas the MOS based on the uncertainty factor used for the derivation of the t-TDI is related to chronic exposure. The differences in exposure duration can be larger than 5, therefore a MOS of 30 for acute exposure to dental materials can be considered sufficiently large.

Duration of exposure is indeed very relevant. With the exception of dialysis patients for which treatment is repeated for long time periods and can be considered similar to chronic exposure, the exposure via medical devices is generally of limited duration (representative of acute to subchronic exposure in a toxicological context), whereas the MOS is based on long term exposure. The application of correction factors to the MOS accounting for the time of exposure ( 3 as used by ECHA) might indicate that MOS around 50 could be sufficiently large, although with a high degree of uncertainties. Nevertheless scenarios relative to multiple treatment to neonates in intensive care units, prolonged medical procedures such as extracorporeal circulation or transplantation/ implantation of an artificial organ in infants (b.w. around $5 \mathrm{~kg}$ ) and long-term exposure of dialysis patients still raise concerns.

The SCENIHR concludes that risk for adverse effects of BPA may exist, when the BPA is directly available for systemic exposure after non-oral exposure routes, especially for neonates in intensive care units, infants undergoing prolonged medical procedures and for dialysis patients. Although the benefit of medical devices has also to be considered, The SCENIHR recommends that where practicable medical devices not leaching BPA should be used. The possibility to replace BPA in these products should be considered against their efficiency in the treatment, as well as the toxicological profile of the alternative materials. 
However, better data on exposure would be beneficial for the refinement of the present risk assessment to be carried out when new data on exposure via medical devices will be available.

\section{Specific answers to the Terms of Reference}

The SCENIHR was requested to assess the following:

1. To determine whether levels of exposure to BPA from the use of the various medical devices containing BPA could give reasons for concern from the health point of view and, if possible, to provide indications on limit values for BPA release from medical devices.

Most of the exposure scenarios via medical devices analysed in the opinion results in an exposure that is below the t-TDI of $4 \mu \mathrm{g} / \mathrm{kg}$ body weight per day based on kidney toxicity as critical endpoint with a $\mathrm{BMDL}_{10}$ of $8.96 \mathrm{mg} / \mathrm{kg}$ b.w./day. However, the internal systemic exposure due to certain medical treatments is higher than the internal exposure resulting from the oral t-TDI, due to the different kinetic behaviour of BPA following the two different routes of exposure. BPA is considered as $100 \%$ systemically bioavailable following parenteral exposure route, whereas the bioavailability of free BPA after oral exposure is much lower (1-10 \% of the ingested dose)

For BPA exposure resulting from the use of medical devices, little information is available and in many cases due to the lack of experimental data, estimates were used. For this reason only the highest value obtained in the estimate was indicatively used.

The estimated highest BPA exposures for different scenarios were: 1) $3000 \mathrm{ng} / \mathrm{kg}$ b.w./day ( $3 \mu \mathrm{g} / \mathrm{kg}$ b.w./day) for prematurely born infants in neonatal intensive care units; 2) $685 \mathrm{ng} / \mathrm{kg}$ b.w./day for prolonged medical procedures (such as extracorporeal circulation or transplantation/ implantation of an artificial organ) in infants (b.w. around $5 \mathrm{~kg}$ ) ; 3) $57 \mathrm{ng} / \mathrm{kg} \mathrm{b.w./day} \mathrm{for} \mathrm{dialysis} \mathrm{patients;} \mathrm{4)} 0.4-12 \mathrm{ng} / \mathrm{kg} \mathrm{b.w./day} \mathrm{for} \mathrm{long}$ term exposures to medical devices; 5) 140 to $200 \mathrm{ng} / \mathrm{kg}$ b.w./day for, respectively, children and adults due to contact with dental materials $(<24 \mathrm{~h}) ; 6) 2$ to $12 \mathrm{ng} / \mathrm{kg}$ b.w./day due to long term contact with dental materials, far below the exposure to BPA via food (EFSA 2013, EFSA 2015).

Concerning exposure via the oral route, it can be concluded that the long term exposure to BPA via dental material is far below the recently derived t-TDI of $4 \mu \mathrm{g} / \mathrm{kg} \mathrm{b.w./day} \mathrm{and}$ poses negligible risk for human health associated to BPA exposure.

Regarding the parenteral exposure via medical devices, considering the differences in bioavailability (assumed as $100 \%$ ) when compared to the oral route (around $1 \%$ ), the SCENIHR considered it appropriate to apply a margin of safety (MOS) approach using the internal dose $(6 \mu \mathrm{g} / \mathrm{kg}$ b.w./day $=\mathrm{HED} / 100)$ rather than the external t-TDI.

Applying a MOS approach, for the $3 \mu \mathrm{g} / \mathrm{kg}$ b.w./day corresponding to medical devices use in neonatal intensive care units the MOS is 2; for prolonged medical procedures in infants the MOS is 10; for the other short-term exposure scenarios estimated for different medical devices, the MOS ranges between 43 and 100; for dialysis treatments the MOS is 105 . For the other scenarios the exposure ranges from $0.4 \mathrm{ng} / \mathrm{kg} \mathrm{b} . w . /$ day to $12 \mathrm{ng} / \mathrm{kg}$ b.w./day resulting in a MOS range from 500 to 15,000.

In relation to exposure to BPA released from dental materials during the first 24 hours after placement, uptake in the oral cavity by oral mucosa can be considerable. Assuming it $100 \%$ as a worst case scenario, for the highest exposures $200 \mathrm{ng} / \mathrm{kg} \mathrm{b} . \mathrm{w} . /$ day the MOS would be 30 . However, the assumption of $100 \%$ bioavailability is largely exaggerated and the peak of exposure occurs for $<24 \mathrm{~h}$ (representative of acute exposure in a toxicological context) whereas the MOS based on the uncertainty factor used for the derivation of the t-TDI is related to chronic exposure. The differences in exposure duration can be larger than 5, therefore a MOS of 30 for acute exposure to dental materials can be considered sufficiently large. 
Duration of exposure is indeed very relevant. With the exception of dialysis patients for which treatment is repeated for long time periods and can be considered equal to chronic exposure, the exposure via medical devices is generally of limited duration (representative of acute to subchronic exposure in a toxicological context). The application of correction factors to the MOS accounting for the time of exposure ( 3 as used by ECHA) might indicate that MOS around 50 could be sufficiently large, although with a high degree of uncertainties. Nevertheless some scenarios still remains in the area of concern..

The SCENIHR concludes that risk for adverse effects of BPA may exist, when the BPA is directly available for systemic exposure after non-oral exposure routes, especially for neonates in intensive care units, infants undergoing prolonged medical procedures and for dialysis patients.

However, it should be underlined that the actual level of exposure is poorly characterised and deserves further investigation. The uncertainties related to the exposure assessment represent the weakest part of this evaluation. Better data on exposure would be beneficial for the refinement of this risk assessment, to be carried out when new data on exposure via medical devices is available. In addition, the uncertainty factor determined by EFSA was derived for a life-long exposure, while for most exposure to medical devices the exposure time is limited.

2. To identify whether any particular medical devices containing BPA could result in human exposures which will give reasons for concern under their normal use patterns or other foreseeable circumstances (e.g. high release of BPA due to the nature of the material of the medical device or to particular contact conditions).

The identification of exposure from medical devices is the weakest part of the evaluation, due to lack of data. More appropriate data on the content and release of BPA from specific medical devices in the actual conditions of use would be beneficial for answering the question in a more quantitative way. However, based on the available information, the highest exposure is estimated to occur in NICU via a multitude of medical devices used, especially in prematurely born infants giving rise to an extremely low MOS. Additionally risks have been highlighted for infants (b.w. $5 \mathrm{~kg}$ ) undergoing prolonged medical procedures such as extracorporeal circulation (by pass) and long term exposure of dialysis patients.

No data are available on the possible exposure to BPA via use of medical devices consisting of BPA-containing PVC. European PVC manufacturers informed the SCENIHR that they no longer use BPA in PVC production, and hence low level of exposure could be expected. However, medical devices (e.g. medical gloves), from non EU-Countries can be marketed: since no information is available on these products, this issue deserves further consideration.

3. To identify, any patient group e.g. infants, pregnant and breastfeeding women who would be particularly at risk in light of the answer to the above questions.

Although not yet unequivocally demonstrated the possible effects observed in animals after prenatal exposure and exposure early in life (i.e.mammary gland proliferation, neurobehavioural development) still remain controversial. Although differences in kinetics between rodents and primates have been demonstrated, indicating a higher internal exposure in newborn rodents than in non human primates at the same level of external exposure, it can be assumed that the premature and the newborn child might be a population at particular risk, especially in view of the high exposure levels resulting from medical devices used. Likewise, dialysis patients are at particular risk, due to the long term exposure and the already malfunctioning of the kidney, a relevant target organ for BPA toxicity. Although the benefit of medical devices has also to be considered, the SCENIHR recommends that where practicable medical devices not leaching BPA should be used. 
4. In case reasons for concern related to BPA are identified, to propose possible alternative approaches that could reduce potential risks either by identifying alternative practices or by identifying alternatives to the use of BPA in medical devices. If no clear answer can be provided on this point, the SCENIHR is asked to formulate recommendations for research that could help provide scientific evidence to that end.

Several alternatives for BPA exist and are increasingly used, notably Bisphenol $S$ and Bisphenol $F$ and some halogenated BPA derivatives (e.g. tetrachlorobisphenol $A$ and tetrabromobisphenol A). For some of the alternatives similar effects as for BPA were reported regarding endocrine activity in in vitro assays, although in general the alternatives had reduced activity/potency when compared to BPA. However, since the endocrine activity has not been identified as the critical effect for BPA, other information should be collected on the effects associated to BPA alternatives. Unfortunately, the toxicological profile of the alternatives to BPA is much less known, and at present it is not possible to compare the potential risk associated with alternatives to the risk due to BPA exposure.

Recommendations for further research especially in the area of exposure through medical devices are presented in section 3.13. Specifically, it could be beneficial to carry out test to identify BPA release in the actual condition of use of the specific medical device, allowing the use of materials with the lowest leaching potential. It is important to underline that the quality of the used medical device can be of relevance. One of the few available studies indicate that BPA total concentrations among neonates in one health care unit were about 17 times higher than those among the infants hospitalized in the second one, undergoing similar treatments. The authors suggested that this difference may be due to the medical devices used, which is supported for the same neonates by a strong association between the levels of di(2-ethylhexyl) phthalate (DEHP) and BPA total concentrations in the urine.

The possibility to replace BPA in medical devices should be considered against the efficiency of the alternatives in the treatment, as well as the toxicological profile of the alternative materials. 


\section{CONSIDERATION OF THE RESPONSES RECEIVED DURING THE CONSULTATION PROCESS}

A public consultation on this opinion was opened on the website of the ScientificCommittees from 29 January 2014 to 26 March 2014. Information about the public consultation was broadly communicated to national authorities, international organisations and other stakeholders.

Fourteen organisations and companies participated in the public consultation providing input to the main scientific questions (in total 119 contributions were received). Out of the 14 organisations participating in the consultation, there were six industry associations, three private companies, two professional organisations, two governments and one non-governmental organization.

Each submission was carefully considered by the SCENIHR and the Opinion has been revised to take account of relevant comments. The literature has been accordingly updated with relevant publications. Notably, two important papers published in 2014 after publication of the preliminary SCENIHR Opinion on BPA in medical devices, were included (Delclos et al., 2014, Churchwell et al., 2014).

The scientific rationale and the opinion section were clarified and strengthened. However, a number of comments received addressed issues which are in the "Draft Scientific Opinion on the risks to public health related to the presence of bisphenol A (BPA) in foodstuffs" (EFSA 2014). More specifically these comments addressed the method used by the EFSA to determine the temporary- Tolerable Daily Intake (t-TDI). The SCENIHR has adopted the temporary oral TDI (t-TDI) of $4 \mu \mathrm{g} / \mathrm{kg}$ b.w./day derived by EFSA which considers all adverse biological effects and takes into account uncertainties (as well as the use of a BPA specific assessment factor of 150). This t-TDI, represents a useful basis for risk assessment for the use of BPA in medical devices.

The text of the comments received and the response provided by the SCENIHR is available at:

http://ec.europa.eu/health/scientific committees/consultations/public consultations/scen ihr consultation 18 en.htm 


\section{MI NORI TY OPI NI ON}

None 


\section{LIST OF ABBREVIATIONS}

AGD

AMPA

ANSES

AR

AUC

BADGE

BASC-SR

BFDGE

BHP

Bis-DMA

Bis-EMA

Bis-GMA

BMD

BMDL

BMDU

BPA

BPAQ

BPB

BPF

$\operatorname{BPS}(U)$

BRCA1

b.w.

CEF

CERHR

Cmax

CMV

CPB

CYP

dBPA

DEHP

DES

DMAB
Anogenital distance

a-amino-3-hydroxy-5-methyl-4-isoxazolepropionic acid (receptor)

Agence nationale de sécurité sanitaire de l'alimentation, de l'environnement et du travail (Agency for Food, Environment and Occupational Health and Safety, Paris, France)

Androgen receptor

Area Under the Curve

Bisphenol A diglycidyl ether

Behavior Assessment System for Children - Self Reported

Bisphenol $\mathrm{F}$ diglycide ether

N-nitrosobis-(2-hydroxypropyl)-amine

Bisphenol A dimethacrylate (2,2-di(4-Methacryloxyphenyl)propane)

Ethoxylated bisphenol A dimethacrylate (2,2-bis(4-(2Methacryloxyethoxy)-phenylpropane)

Bisphenol A glycidyl methacrylate

Bench Mark Dose

Bench Mark Dose Low (lower value of $90 \%$ confidence interval of BMD)

Bench Mark Dose Upper (upper value of $90 \%$ confidence interval of BMD)

Bisphenol A, Bis(4-hydroxyphenyl)propane

BPA-3,4-quinone

Bisphenol B, 2,2-Bis(4-hydroxyphenyl)butane

Bisphenol F, Bis(4-hydroxydiphenyl)methane

Bis(4-hydroxyphenyl)sulfone

Breast Cancer 1 (gene)

body weight

Panel on Food Contact Materials, Enzymes, Flavourings and Processing Aids (EFSA panel)

Center for the Evaluation of Risks to Human Reproduction (USA)

Maximum concentration

Cytomegalovirus

Cardiopulmonary bypass

Cytochrome P450

deuterated BPA

Di(2-ethylhexyl) phthalate

Di-ethylstilbestrol

3,2-dimethyl-4-aminobiphenyl 


\begin{tabular}{|c|c|}
\hline DMBA & 7,12-Dimethylbenz(a)anthracene \\
\hline DMSO & Dimethyl Sulfoxide \\
\hline DNA & Deoxyribonucleic acid \\
\hline DNMT & DNA methyltransferase \\
\hline DSP & Daily sperm production \\
\hline EB & $17 ß$-estradiol-3-benzoate \\
\hline ECB & European Chemicals Bureau \\
\hline ECDC & European Centre for Disease prevention and Control \\
\hline $\mathrm{ECHA}$ & European Chemicals Agency \\
\hline ECMO & Extracorporeal membrane oxygenation \\
\hline $\mathrm{EE}$ & Ethinyl Estradiol \\
\hline EFSA & European Food Safety Authority \\
\hline ELISA & Enzyme-Linked Immuno Sorbent Assay \\
\hline EMA & European Medicines Agency \\
\hline ENNG & $\mathrm{N}$-ethyl-N' -nitro-nitrosoguanidine \\
\hline EPM & Elevated Plus Maze \\
\hline ER & Estrogen Receptor \\
\hline EtO & Ethylene oxide \\
\hline EU-RAR & EU Risk Assessment Report \\
\hline FAO & Food and Agriculture Organization \\
\hline FDA & Food and Drug Administration (USA) \\
\hline FST & Forced Swimming Test \\
\hline GC & Gas Chromatography \\
\hline GC-MS & Gas Chromatography-Mass Spectrometry \\
\hline GD & Gestational Day \\
\hline GI & Gastro-intestinal \\
\hline GLP & Good Laboratory Practices \\
\hline $\mathrm{HCA}$ & Hydroxycumyl alcohol \\
\hline HDL & High-density lipoprotein \\
\hline HED & Human Equivalent Dose \\
\hline HPG & hypothalamic-pituary-gonadal (axis) \\
\hline HPLC-MS & High Pressure Liquid Chromatography-Mass Spectrometry \\
\hline HPLC/UV-DAD & $\begin{array}{l}\text { High Pressure Liquid Chromatography/Ultraviolet-Diode } \\
\text { Detector }\end{array}$ \\
\hline ICU & Intensive Care Unit \\
\hline IPCS & International Programme on Chemical Safety \\
\hline ISO & International Organization for Standardization \\
\hline i.v. (IV) & intraveneously \\
\hline LC & Liquid Chromatography \\
\hline
\end{tabular}




\begin{tabular}{|c|c|}
\hline LC-ECD & Liquid Chromatography-ElectroChemical Detector \\
\hline LC-MS & Liquid Chromatography-Mass Spectrometry \\
\hline LD50 & Lethal Dose at which $50 \%$ of the animals die \\
\hline LDL & Low density lipoprotein \\
\hline LOAEL & Lowest Observed Adverse Effect Level \\
\hline LOD & Limit of Detection \\
\hline LOQ & Limit of Quantification \\
\hline MBP & 4-methyl-2,4-bis(4-hydroxyl-phenyl) pent-1-ene \\
\hline MOS & Margin of Safety \\
\hline MRI & Magnetic Resonance Imaging \\
\hline mRNA & Messenger Ribonucleic Acid \\
\hline MS & Mass Spectrometry \\
\hline MTD & Maximum Tolerated Dose \\
\hline MWM & Morris Water Maze \\
\hline NHANES & National Health and Nutrition Examination Survey (USA) \\
\hline NICU & Neonatal Intensive Care Unit \\
\hline NIEHS & National Institute of Environmental Health Sciences (USA) \\
\hline NOAEL & No Observed Adverse Effect Level \\
\hline NCTR & National Center for Toxicological Research (USA) \\
\hline NHP & Non Human Primates \\
\hline NMDA & N-Methyl-d-Aspartate (receptor) \\
\hline NMU & N-nitroso- N-methylurea \\
\hline NTP & National Toxicology Program (USA) \\
\hline NTP-CERHR & $\begin{array}{l}\text { National Toxicology Program-Center for the Evaluation of Risks to } \\
\text { Human Reproduction (USA) }\end{array}$ \\
\hline OECD & Organisation for Economic Co-operation and Development \\
\hline $8-\mathrm{OHdG}$ & 8-hydroxydeoxyguanosine \\
\hline OP & Object Placement \\
\hline OR & Object Recognition \\
\hline OVX & Ovariectomised \\
\hline PBPK & Physiologically Based Pharmacokinetic (modeling) \\
\hline PC & Polycarbonate \\
\hline PEPA & Polyester-polymeralloy \\
\hline PIN & Prostatic intraepithelial neoplasia \\
\hline PND & Post Natal Day \\
\hline ppm & parts per million (translates into $\mathrm{mg} / \mathrm{L}$ or $\mathrm{ng} / \mathrm{mg}$ ) \\
\hline PPAR & Peroxisome proliferator-activated receptor \\
\hline PSD & Postsynaptic density \\
\hline PSU & Polysulfone \\
\hline
\end{tabular}




\begin{tabular}{ll}
\hline PVC & Polyvinyl chloride \\
pWAT & Perigonadic white adipose tissue \\
REACH & $\begin{array}{l}\text { Registration, Evaluation, and Authorisation of Chemicals (EU } \\
\text { Regulation) }\end{array}$ \\
RIA & Radioimmunoassay \\
RNA & Ribonucleic Acid \\
S.C. (SC) & subcutaneous \\
SCCS & Scientific Committee on Consumer Safety \\
SCENIHR & Scientific Committee on Emerging and Newly Identified Health Risks \\
SCHER & Scientific Committee on Health and Environmental Risks \\
SE & Standard Error \\
SPE & Solid-phase extraction \\
SULT & Sulfotransferase \\
TBBPA & Tetrabromobisphenol-A [2,2-bis(4-hydroxy-3,5- \\
TCBPA & dibromophenyl)propane] \\
TDI & Tetrachlorobisphenol-A [2,2-bis(4-hydroxy-3,5- \\
T1DM & dichlorophenyl)propane] \\
TEGDMA & Tolerable Daily Intake \\
UDPGT & Type 1 diabetes mellitus \\
UGT & Triethylene glycol dimethacrylate \\
UPLC & Uridinediphosphate- glucuronosyltransferase \\
WHO & UDP-glucuronyltransferase \\
& Ultra Performance Liquid Chromatography \\
World Health Organization
\end{tabular}




\section{REFERENCES}

Aalto-Korte K, Alanko K, Henriks-eckerman ML, Estlander T, Jolanki R. (2003) Allergic contact dermatitis from bisphenol A in PVC gloves - Contact Dermatitis, 49, 202-205.

Acevedo N, Davis B, Schaeberle CM, Sonnenschein C and Soto AM. (2013) Perinatally Administered Bisphenol A Acts as a Mammary Gland Carcinogen in Rats. Environmental Health Perspectives, 121, 1040-1046

Alonso-Magdalena P, Morimoto S, Ripoll C, Fuentes E, Nadal A. (2006) Environ Health Perspect. The estrogenic effect of bisphenol A disrupts pancreatic beta-cell function in vivo and induces insulin resis $\neg$ tance. 114, $106-112$.

Alonso-Magdalena P, Vieira E, Soriano S, Menes L, Burks D, Quesada I, Nadal A (2010) Bisphenol-A exposure during pregnancy disrupts glucose homeostasis in mothers and adult male offspring. Environmental Health Perspectives 118, 1243-1250.

Alonso-Magdalena P, Quesada I, Nadal A. (2011) Endocrine disruptors in the etiology of type 2 diabetes mellitus. Nat Rev Endocrinol 7, 346-353.

Anderson OS, Peterson KE, Sanchez BN, Zhang Z, Mancuso P, Dolinoy DC. (2013) Perinatal bisphenol A exposure promotes hyperactivity, lean body composition, and hormonal responses across the murine life course. FASEB J. 27, 1784-1792.

Angle BM, Do RP, Ponzi D, Stahlhut RW, Drury BE, Nagel SC, Welshons WV, BeschWilliford CL, Palanza P, Parmigiani S, Vom Saal FS, Taylor JA. (2013) Metabolic disruption in male mice due to fetal exposure to low but not high doses of bisphenol A (BPA): Evidence for effects on body weight, food intake, adipocytes, leptin, adiponectin, insulin and glucose regulation. Reprod Toxicol. 2013 Jul 17. pii: S0890-6238(13)00231-1. doi: 10.1016/j.reprotox.2013.07.017. [Epub ahead of print]).

ANSES (2011) Health effects of Bisphenol A. Request nos. 2009-SA-0331 and 2010-SA0197. Collective Expert Report. ANSES (Agence National de Securite Sanitaire Alimentation, Environment, Travail, French Agency for Food, Environmental and Occupational Health \& Safety) Paris, France, September 2011.

ANSES (2013) Evaluation des risques du bisphenol A (BPA) pour la santé humaine. Substances reprotoxiques et perturbateurs endocrines. (Agence National de Securite Sanitaire Alimentation, Environment, Travail, French Agency for Food, Environmental and Occupational Health \& Safety, Paris, France), Paris, France, Mars 2013. http://www.anses.fr/fr/content/evaluation-des-risques-sanitaires-li\%C3\%A9s-aubisph\%C3\%A9nol

Arenholt-Bindslev D, Breinholt V, Preiss A, Schmalz G.(1999) Time-related bisphenol-A content and estrogenic activity in saliva samples collected in relation to placement of fissure sealants. Clinical Oral Investigations 3, 120-125, 1999.

Arnich N, Canivenc-Lavier M.C, Kolf-Clauw M, Coffigny H, Cravedi J.P, Grob K, Macherey A.C, Masset D, Maximilien R, Narbonne J.F, Nesslany F, Stadler J, Tulliez J. (2011) Conclusions of the French Food Safety Agency on the toxicity of bisphenol A. Int J Hyg Environ Health 214, 271-275.

Aschberger K, Castello P, Hoeskstra E, Karakitsios S, Munn S, Pakalin S, Sarigiannis D. (2010). Bisphenol A and baby bottles: challenges and perspectives- JRC- European Commission EUR 24389.

Ashby J, Tinwell H, Haseman J. (1999) Lack of effects for low dose levels of bisphenol A and diethylstilbestrol on the prostate gland of CF1 mice exposed in utero. Regul Toxicol Pharmacol 30, 156-166.

Audebert M, Dolo L, Perdu E, Cravedi JP, Zalko D. (2011) Use of the YH2AX assay for assessing the genotoxicity of bisphenol $A$ and bisphenol $F$ in human cell lines. Arch Toxicol. 85, 1463-1473. 
Ayyanan A, Laribi O, Schuepbach-Mallepell S, Schrick C, Gutierrez M, Tanos T, Lefebvre G, Rougemont J, Yalcin-Ozuysal O, Brisken C. (2011) Perinatal exposure to bisphenol a increases adult mammary gland progesterone response and cell number. Mol Endocrinol. 25, 1915-1923.

Baker DA, Hastings RS, Pruitt L (2000): Composition and tension resistance of medical grade ultra-high molecular weight polyethylene; the effect of morphology, sterilization, aging and temperature. Polymer 200041 (2) 795-808.

Balakrishnan B, Henare K, Thorstensen EB, Ponnampalam AP, and Mitchell MD, 2010. Transfer of bisphenol A across the human placenta. American Journal of Obstetrics and Gynecology 202, 393.e1-7.

Batista TM, Alonso-Magdalena P, Vieira E, Amaral ME, Cederroth CR, Nef S, Quesada I, Cameiro EM, Nadal A. (2012) Short-term treatment with bisphenol-A leads to metabolic abnormalities in adult male mice. PLoS One 7 (3), e33814.

Bauer SM, Roy A, Emo J, Chapman TJ, Georas SN, Lawrence BP. (2012) The effects of maternal exposure to bisphenol $A$ on allergic lung inflammation into adulthood. Toxicol Sci 130, 82-93

Becker K, Güen T, Seiwert M (2009) GerES IV: phthalate metabolites and bisphenol A in urine of German children. Internat J Hyg Environ Health. 212: 685-692.

Begley T, Castle L, Feigenbaum A, Franz R, Hinrichs K, Lickly T, Mercea P, Milana M, O'Brien A, Rebre S, Rijk R, Piringer O. et al. (2005). Evaluation of migration models in support of regulations for food-contact plastics. Food Addit Contam 22: 73:90.

Begley T.H, Dennison J.L, Hollifield H.C. (1990). Migration into food of polyethylene terephthalate (PET) cyclic oligomers from PET microwave packaging. Food Addit Contam 7: 797:803.

Beronius A and Hanberg A (2011) Sources of exposure to bisphenol A. IMM report 2/2011, Karolinska Institut, Stockholm, Sweden. http://ki.se/content/1/c6/12/58/71/IMMrapport2-2011.pdf

Betancourt AM, Eltoum IA, Desmond RA, Russo J, Lamartiniere CA. (2010) In utero exposure to bisphenol A shifts the window of susceptibility for mammary carcinogenesis in the rat. Environ Health Perspect. 118, 1614-1619.

Biedermann S, Tschudin P, Grob K. (2010) Transfer of bisphenol A from thermal printer paper to the skin. Analytical and Bioanalytical Chemistry 398, 571-576 (2010).

Biles JE, McNeal TP, Begley TH, Hollifield HC (1997). Determination of Bisphenol-A in Reusable Polycarbonate Food-Contact Plastics and Migration to Food-Simulating Liquids. Journal of Agricultural and Food Chemistry 45: 3541-3544. Corrected in J. of Agric. Food Chem. 46:2894.

Bloom MS, Vom Saal FS, Kim D, Taylor JA, Lamb JD, Fujimoto VY. (2011) Serum unconjugated bisphenol $A$ concentrations in men may influence embryo quality indicators during in vitro fertilization. Environ Toxicol Pharmacol. 32, 319-323.

Bodin J, Bølling AK, Samuelsen M, Becher R, Løvik M, Nygaard UC. (2013) Long-term bisphenol A exposure accelerates insulitis development in diabetes-prone NOD mice. Immunopharmacol Immunotoxicol. 35, 349-358.

Borrell B. (2010) Toxicology: The big test for bisphenol A. Nature. 464, 1122-1124.

Braun JM, Yolton K, Dietrich KN, Homung R, Ye X, Calafat Am, Lanphear BP. (2009) Prenatal bisphenol A exposure and early childhood behavior. Environ Health Perspect 117, 1945-1952.

Braun JM, Kalkbrenner AE, Calafat AM, Yolton K, Ye X, Dietrich KN, Lanphear BP. (2011) Impact of early-life bisphenol $A$ exposure on behavior and executive function in children. Pediatrics. 128, :873-882. 
Brown SA, Merritt K, woods T.O, Mc Namees S.G, Hitchins VM (2002). Effect of disinfection and sterilization methods on tensile strength of material used for single use devices. Biomed Instrum. Technol. 36, 23-27.

Bushnik T, Haines D, Levallois P, Levesque J, Van Oostdam J and Viau C (2010) Lead and bisphenol A concentrations in the Canadian population. Health Reports 21: 7-18.

Cabaton N, Dumont C, Severin I, Perdu E, Zalko D, Cherkaoui-MAlki M, Chagnon MC. (2009) Genotoxic and endocrine activities of bis(hydroxyphenyl)methane (bisphenol F) and its derivatives in the HepG2 cell line. Toxicology 255, 15-24.

Cacho JI, Campilo N, Vinas P and Hernandez-Cordoba M (2013) Stir bar sorptive extraction with EG-Silicone coating for bisphenols determination in personal care products by GC-MS. J Pharm Biomed Anal 79: 255-260.

Cagen SZ, Waechter JM Jr, Dimond SS, Breslin WJ, Butala JH, Jekat FW, Joiner RL, Shiotsuka RN, Veenstra GE, Harris LR.(1999) Normal reproductive organ development in CF-1 mice following prenatal exposure to bisphenol A. Toxicol Sci. 50, 36-44, 1999.

Calafat AM, Kuklenyik Z, Reidy J A, Caudill S P, Ekong J and. Needham LL (2005) Urinary concentrations of Bisphenol $A$ and 4-Nonylphenol in a human reference population. Environ Health Perspect 113: 391-395.

Calafat AM, Weuve J, Ye XY, Jia LT, Hu H, Ringer S, Huttner K, Hauser R, (2009). Exposure to bisphenol $A$ and other phenols in neonatal intensive care unit premature infants. Environmental Health Perspectives 117, 639-644.

Calafat AM, Needham LL. (2009) What additional factors beyond state-of-the-art analytical methods are needed for optimal generation and interpretation of biomonitoring data? Environ Health Perspect. 117, 1481-1485.

Calafat AM, Ye X, Wong L-Y, Reidy JA, Needham LL. (2008). Exposure of the U.S. Population to Bisphenol A and 4-tertiary-Octylphenol: 2003-2004. Environmental Health Perspectives , 116, 39-44.

Campbell NR, Van Loon JA and Weinshilboum RM (1987). Human liver phenol sulfotransferase: Assay conditions, biochemical properties and partial purification of isozymes of the thermostable form. Biochemical Pharmacology 36:1435-1446.

Cao J, Mickens JA, McCaffrey KA, Leyrer SM, Patisaul HB. (2012) Neonatal Bisphenol A exposure alters sexually dimorphic gene expression in the postnatal rat hypothalamus. Neurotoxicology. 33, 23-36.

Cao J, Rebuli ME, Rogers J, Todd KL, Leyrer SM, Ferguson SA, Patisaul HB. (2013) Prenatal bisphenol a exposure alters sex-specific estrogen receptor expression in the neonatal rat hypothalamus and amygdala. Toxicol Sci. 133, 157-173.

Cariot A, Dupuis A, Albouy-Llaty M, Legube B, Rabouan S, Migeot V (2012). Reliable quantification of bisphenol $A$ and its chlorinated derivatives in human breast milk using UPLC-MS/MS method. Talanta 100: 175-182.

Carr R, Bertasi F, Betancourt S, Bowers S, Gandy BS, Ryan P, Willard S. (2003) Effect of neonatal rat bisphenol $A$ exposure on performance in the Morris Water Maze. J Toxicol Environ Health A 66, 2077-2088.

Carwile J.L., Michels K.B. (2011) Urinary bisphenol A and obesity: NHANES 2003-2006. Environ Res. 111, 825-830.

Casas M, Valvi D, Luque N, Ballesteros-Gomez A, Carsin AE, Fernandez MF, Koch HM, Mendez MA, Sunyer J, Rubio S, Vrijheid M. (2013) Dietary and sociodemographic determinants of bisphenol $A$ urine concentrations in pregnant women and children. Env Int $56,10-18$. 
Changkhamchom S, Sirivat A, (2010). Synthesis and properties of sulfonated poly(ether ketone ether sulfone) (S-PEKES) via bisphenol S: Effect of sulfonation. Polymer Bulletin, 65, 265-281.

Chapin R, Adams J, Boekelheide K, Gray L, Hayward S, Lees P, McIntyre B, Portier K, Schnorr T, Selevan S, Vandenberg J, Woskie S (2007) NTP-CERHR Expert Panel Report on the Reproductive and Developmental Toxicity of Bisphenol A. http://cerhr.niehs.nih.gov/chemicals/bisphenol/bisphenol.html

Chapin RE, Adams J, Boekelheide K, Gray LE Jr, Hayward SW, Lees PS, McIntyre BS, Portier KM, Schnorr TM, Selevan SG, Vandenbergh JG, Woskie SR (2008). NTP-CERHR expert panel report on the reproductive and developmental toxicity of bisphenol A. Birth Defects Res B Dev Reprod Toxicol. 83, 157-395

Chen MY, Ike M, Fujita M. (2002). Acute toxicity, mutagenicity, and estrogenicity of bisphenol-A and other bisphenols. Environ Toxicol 17:80-86.

Cho S., Choi Y.S., Luu H. and Guo J. (2012). Determination of total leachable bisphenol A from polysulfone membranes based on multiple consecutive extractions. Talanta 101; $537-540$

Chou WC, Chen JL, Lin CF, Chen YC, Shih FC, Chuang CY. (2011). Biomonitoring of bisphenol $A$ concentrations in maternal and umbilical cord blood in regard to birth outcomes and adipokine expression: A birth cohort study in Taiwan. Environ Health 10:94.

Christensen KL, Lorber M, Koch HM, Kolossa-Gehring M, Morgan MK. (2012) Population variability of phthalate metabolites and bisphenol A concentrations in spot urine samples versus 24- or 48-h collections. J Expo Sci Environ Epidemiol. 22, 632-640.

Churchwell MI, Camacho L, Vanlandingham MM, Twaddle NC, Sepehr E, Delclos KB, Fisher JW, Doerge DR (2014) Comparison of life-stage-dependent internal dosimetry for bisphenol $A$, ethinyl estradiol, a reference estrogen, and endogenous estradiol to test an estrogenic mode of action in Sprague Dawley rats. Tox Sc 139, 4-20.

Cichna-Markl M. (2012) Sample clean-up by sol-gel immunoaffinity chromatography for the determination of bisphenol A in food and urine Methods 56 (2012) 186-191

Clayton EM, Todd M, Dowd JB, Aiello AE. (2011) The impact of bisphenol A and triclosan on immune parameters in the U.S. population, NHANES 2003-2006. Environ Health Perspect 119, 390-396.

Cobellis L, Colacurci N, Trabucco E, Carpentiero C, Grumetto L. (2009) Measurement of bisphenol $A$ and bisphenol $B$ levels in human blood sera from healthy and endometriotic women. Biomed Chromatogr 23, 1186-1190.

COM (Committee on Mutagenicity of Chemicals in Food, Consumer Products and the Environment) Guidance on a Strategy for Testing of Chemicals for Mutagenicity, Department of Health, UK, 2000 http://www.iacom.org.uk/guidstate/documents/COMGuidanceFINAL2.pdf

Coughtrie MW, Burchell B, Leakey JE, Hume R. (1988) The inadequacy of perinatal glucuronidation: immunoblot analysis of the developmental expression of individual UDPglucuronosyltransferase isoenzymes in rat and human liver microsomes. Mol Pharmacol 34, 729-735.

Cousins IT, Staples CA, Klečka GM, Mackay D. 2002. A multimedia assessment of the environmental fate of bisphenol A. Human and Ecological Risk Assessment (HERA). 8: 1107-1136

Cox KH, Gatewood JD, Howeth C, Rissman EF. (2010) Gestational exposure to bisphenol $A$ and cross-fostering affect behaviors in juvenile mice. Horm Behav 58, 754-761. 
D'Cruz SC, Jubendradass R, Jayakanthan M, Rani SJ, Mathur PP. (2012) Bisphenol A impairs insulin signaling and glucose homeostasis and decreases steroidogenesis in rat testis: an in vivo and in silico study. Food Chem Toxicol. 50, 1124-1133.

De Flora S, Micale RT, La Maestra S, Izzotti A, D'Agostini F, Camoirano A, Davoli SA, Troglio MG, Rizzi F, Davalli P, Bettuzzi S. (2011) Upregulation of clusterin in prostate and DNA damage in spermatozoa from bisphenol A-treated rats and formation of DNA adducts in cultured human prostatic cells. Toxicol Sci.122, 45-51.

Delclos KB, Camacho L, Lewis SM, Vanlandingham MM, Latendresse JR, Olson GR, Davis KJ, Patton RE, Gamboa da Costa G, Woodling KA, Bryant MS, Chidambaram M, Trbojevich R, Juliar BE, Felton RP, ,Thorn BT. (2014) Toxicity evaluation of bisphenol A administered by gavage to Sprague Dawley Rats from gestation day 6 through postnatal day 90. Tox Sc. 139, 174-197.

Dekant W and Völkel W (2008) Human exposure to bisphenol A by biomonitoring: methods, results and assessment of environmental exposures. Toxicol App Pharmacol 228, 114-134.

De Meulenaer B. and Huyghebaert A. (2004). Packaging and other food contact materials residues. In: Handbook of Food Analysis, Vol.2 (Nollet LML ed) 2nd ed. New York: Marcel Dekker, 1297-1330

Demierre AL, Peter R, Oberli A, Bourqui-Pittet M. (2012) Dermal penetration of bisphenol $A$ in human skin contributes marginally to total exposure. Toxicol Lett. 213, 305-308.

De Wit CA, Herzke D, Vorkamp K. (2010) Brominated flame retardants in the Arctic environment--trends and new candidates. Sci Total Environ 408, 2885-2918.

Diaz Weinstein S, Villafane JJ, Juliano N, Bowman RE. (2013) Adolescent exposure to Bisphenol A increases anxiety and sucrose preference but impairs spatial memory in rats indenpendent of sex. Brain Res 1529, 56-65.

Dobrzyńska MM, Radzikowska J. (2013) Genotoxicity and reproductive toxicity of bisphenol $A$ and $X$-ray/bisphenol A combination in male mice. Drug Chem Toxicol. 36, 19-26.

Dodson RE, Nishioka M, Standley LJ, Perovich LJ, Brody JG and Rudel RA (2012) Endocrine disruptors and asthma-associated chemicals in consumer products. Environmental Health Perspectives 120, 935-944.

Doerge DR, Twaddle NC, Vanlandingham M and Fisher JW, (2010a). Pharmacokinetics of bisphenol $A$ in neonatal and adult Sprague-Dawley Rats. Toxicology and Applied Pharmacology 247, 158-165.

Doerge DR, Twaddle NC, Vanlandingham M and Fisher JW, (2011a) Pharmacokinetics of Bisphenol $A$ in neonatal and adult CD-1 mice: Inter-species comparisons with SpragueDawley rats and rhesus monkeys Toxicology Letters 207, 298- 305.

Doerge DR, Twaddle NC, Vanlandingham M and Fisher JW, (2012) Pharmacokinetics of bisphenol $A$ in serum and adipose tissue following intravenous administration to adult female CD-1 mice Toxicology Letters 211, 114- 119.

Doerge DR, Twaddle NC, Woodling KA and Fisher JW, (2010b). Pharmacokinetics of bisphenol $a$ in neonatal and adult rhesus monkeys. Toxicology and Applied Pharmacology $248,1-11$

Doerge D R, Vanlandingham M, Twaddle N C, Delclos K B (2010c). Lactational transfer of bisphenol A in Sprague-Dawley rats. Toxicol Lett 199, 372-376.

Doerge, D.R., Twaddle, N.C., Vanlandingham, M., Fisher, J.W., (2011b). Distribution of bisphenol A into tissues of adult neonatal, and fetal Sprague-Dawley rats. Toxicol. Appl. Pharmacol. 255, 261-270 
Dolinoy DC, Huang D and Jirtle RL, (2007). Maternal nutrient supplementation counteracts bisphenol A-induced DNA hypomethylation in early development. Proceedings of the National Academy of Sciences of the United States of America 104, 13056-13061.

Donohue KM, Miller RL, Perzanowski MS, Just AC, Hoepner LA, Arunajadai S, Canfield S, Resnick D, Calafat AM, Perera FP, Whyatt RM (2013) Prenatal and postnatal bisphenol A exposure and asthma development among inner-city children. J Allergy Clin Immunol, $131,736-742$.

Duanmu Z, Weckle A, Koukouritaki SB, Hines RN, Falany Jl, Falany CN, Kocarek TA and Runge-Morris M. (2006). Developmental expression of aryl, estrogen and hydoxysteroid sulfotransferases in pre- and post-natal human liver. Journal of Pharmacology and Experimental Therapeutics 316, 1310-1317.

Durando M, Kass L, Piva J, Sonnenheim C, Soto AM, Luque EH, Munoz-de-Toro M. (2007) Prenatal bisphenol A exposure induces preneoplastic lesions in the mammary gland in wistar rats. Envir Health Perspect 115, 80-86.

ECB, European Chemicals Bureau (2003). European Union Risk Assessment Report: 4,4' ISOPROPYLIDENEDIPHENOL (Bisphenol-A). CAS No:80-05-7. Institute for Health and Consumer Protection, European Chemicals Bureau, European Commission Joint Research Centre, 3rd Priority List, Luxembourg: Office for Official Publications of the European Communities

http://publications.jrc.ec.europa.eu/repository/bitstream/111111111/1304/1/EUR\%2020 $843 \% 20$ EN.pdf

ECB (2004) European Commission Scientific Committee on toxicity, ecotoxicity and the environment (CSTEE). Opinion on the results of a second Risk Assessment of: bis(2ethylhexyl)phthalate [DEHP]: Human Health Part. CAS No.: 117-81-7; EINECS No.: 204211-0. Adopted by the CSTEE during the 41th plenary meeting of 8 January 2004.

EC (2008) European Union Risk Assessment Report Human Helath Addendum of April 2008 (to be read in conjunction with published EU RAR of BPA 2003) 4,4'Isopropylidenediphenol (Bisphenol-A) Part 2 Human Health. http://publications.jrc.ec.europa.eu/repository/bitstream/111111111/15069/1/lbna2458 9enn.pdf

EC, European Commission (2010b) European Union Risk Assessment Report 4,4'ISOPROPYLIDENEDIPHENOL (Bisphenol-A), Part 1 Environmental Health - Environment Addendum of April 2008 (to be read in conjunction with published EU RAR of BPA, 2003) http://publications.jrc.ec.europa.eu/repository/bitstream/111111111/15063/1/lbna2458 8enn.pdf

EC, European Commission (2010a) European Union Risk Assessment Report 4,4'ISOPROPYLIDENEDIPHENOL (Bisphenol-A), Part 2 Human Health - Human Health Addendum of April 2008 (to be read in conjunction with published EU RAR of BPA, 2003) http://publications.jrc.ec.europa.eu/repository/bitstream/111111111/15069/1/lbna2458 9enn.pdf

ECHA (2014), Committee for Risk Assessment (RAC). ECHA/PR/14/07. RAC proposes to strengthen the classification of bisphenol A.

http://echa.europa.eu/view-article/-/journal_content/title/rac-proposes-to-strengthenthe-classification-of-bisphenol-a

Edginton A N, Ritter L (2009) Predicting plasma concentrations of bisphenol A in children younger than 2 years of age after typical feeding schedules, using a physiologically based toxicokinetic model. Environmental Health Perspectives 117, 645-652.

Edlow AG, Chen M, Smith NA, Lu C and McElrath TF. (2012) Fetal bisphenol A exposure: concentration of conjugated and unconjugated bisphenol $A$ in amniotic fuid in the second and third trimesters. Reproductive Toxicology, 34, 1-7. 
EFSA (2006). Opinion of the Scientific Panel on food additives, flavourings, processing aids and materials in contact with food (AFC) related to 2,2-BIS(4-HYDROXYPHENYL) PROPANE (Bisphenol A). The EFSA Journal 428, 1- 75. http://www.efsa.europa.eu/EFSA/efsa_locale-1178620753812_1178620772817.htm

EFSA (2009) Guidance of the Scientific Committee on a request from EFSA on the use of the bench mark dose approach in risk assessment. The EFSA Journal 1150, 1-72. http://www.efsa.europa.eu/it/efsajournal/doc/1150.pdf

EFSA (2010). Scientific Opinion of the Panel on food contact materials, enzymes, flavourings and processing aids (CEF) on Bisphenol A: evaluation of a study investigating its neurodevelopmental toxicity, review of recent scientific literature on its toxicity and advice on the Danish risk assessment of Bisphenol A. The EFSA Journal 8, 1829. http://www.efsa.europa.eu/en/efsajournal/doc/1829.pdf

EFSA (2011) Technical Report. Use of BMDS and PROAST software packages by EFGSA scientific panels and units for applying the Bench MRK Dose (BMD) approach in risk assessment. European Food Safety Authority (EFSA), Parma, Italy. http://www.efsa.europa.eu/en/supporting/doc/113e.pdf

EFSA (2012a) EFSA Scientific Committee. Guidance on selected default values to be used by the EFSA Scientific Committee, Scientific Panels and Units in the absence of actual $\begin{array}{lllll}\text { measured data. Journal } & 2579 .\end{array}$ http://www.efsa.europa.eu/en/efsajournal/doc/2579.pdf

EFSA (2012b) EFSA panel on plant protection products and their residues (PPR). Scientific Opinionon; Guidance on dermal absorption. EFSA Journal 10, 2665.

EFSA (2013). DRAFT Scientific Opinion on the risks to public health related to the 4 presence of bisphenol A (BPA) in foodstuffs - Part: exposure assessment. Draft Scientific Opinion Endorsed for Public Consultation. http://www.efsa.europa.eu/en/consultations/call/130725.pdf

EFSA (2014) Draft Scientific Opinion on the risks to public health related to the presence of bisphenol A (BPA) in foodstuffs. ENDORSED FOR PUBLIC CONSULTATION DRAFT SCIENTIFIC OPINION. EFSA Panel on Food Contact Materials, Enzymes, Flavourings and Processing Aids (CEF), European Food Safety Authority (EFSA), Parma, Italy. January 2014.

EFSA (2015) Scientific Opinion on the risks to public health related to the presence of bisphenol A (BPA) in foodstuffs. Part II - Toxicological assessment and risk characterisation. EFSA Panel on Food Contact Materials, Enzymes, Flavourings and Processing Aids (CEF), European Food Safety Authority (EFSA), Parma, Italy. EFSA Journal 13, 3978. http://www.efsa.europa.eu/en/efsajournal/doc/3978part2.pdf

Eilam-Stock T, Serrano P, Frankfurt M, Luine V (2012) Bisphenol-A impairs memory and reduces dendritic spine density in adult male rats. Behavioral Neuroscience, 126, 175185.

Eliades T, Hiskia A, Eliades G and Athanasiou AE (2007) Assessment of bisphenol-A release from orthodontic adhesives. Am J Orthod Dentofacial Orthop 131: 72-75

Eliades T, Voutsa D, Sifakakis I, Makou M and Katsaros C (2011) Release of bisphenol-A from a light-cured adhesive bonded to lingual fixed retainers. Am J Orthod Dentofacial Orthop 139: 192-195

Ema M, Fujii S, Furukawa M, Kiguchi M, Ikka T, Harazono A. (2001) Rat two-generation reproductive toxicity study of bisphenol A. Reprod Toxicol. 15, 505-523.

Environment Canada/Health Canada (2008) Screening Assessment for the Challenge Phenol, 4,4' -(1-methylethylidene)bis- (Bisphenol A). Available on-line at: http://www.ec.gc.ca/ese-ees/3C756383-BEB3-45D5-B8D3-E8C800F35243/batch2_8005-7_en.pdf 
FAO/WHO (2011) Toxicological and Health Aspects of Bisphenol A. Report of Joint FAO/WHO Expert Meeting. World Health Organization. 2011. ISBN 97892141564274. http://www.who.int/foodsafety/chem/chemicals/BPA_Summary2010.pdf

Ferguson SA, Law CD, Abshire JS. (2012) Developmental treatment with bisphenol A causes few alterations on measures of postweaning activity and learning. Neurotoxicol Teratol. 34, 598-606.

Fernandez MF, Arrebola JP, Taoufiki J, Navalon A, Ballesteros O, Pulgar R, Vilchez J L, Olea N, (2007). Bisphenol-A and chlorinated derivatives in adipose tissue of women. Reproductive Toxicology 24, 259-264.

Fink K (2008). Toxins in Renal Disease and Dialysis Therapy: Genotoxic Potential and Mechanisms. Dissertation zur Erlangung des naturwissenschaftlichen Doktorgrades der Bayerischen Julius-Maximilians-Universität Würzburg.

Fisher JW, Twaddle NC, Vanlandingham M, Doerge DR (2011) Pharmacokinetic modeling: prediction and evaluation of route-dependent dosimetry of bisphenol $A$ in monkeys with extrapolation to humans. Toxicol. Appl. Pharmacol., 257 (2011) 122-136.

Fleisch AF, Sheffield PE, Chinn C, Edelstein BL, Landrigan PJ (2010) Bisphenol A and Related Compounds in Dental Materials. Pediatrics 126:760-768.

Fromme H. Küchlerb T, Ottoc T, Pilzc K, Müllerb J, Wenzel A. (2002). Occurrence of phthalates and bisphenol $A$ and $F$ in the environment. Water Res. 36, 1429-1438.

Fu P, Kawamura K (2010) Ubiquity of bisphenol A in the atmosphere. Environmental Pollution 158: 3138-3143.

Fujimoto T, Kubo K, Aou S.(2006) Prenatal exposure to bisphenol A impairs sexual differentiation of exploratory behavior and increases depression-like behavior in rats. Brain Res. 1068, 49-55.

Fujimoto T, Kubo K, Nishikawa Y, Aou S (2013) Postnatal exposure to low-dose bisphenol A influences various emotional consitions. J Toxicol Sc 38, 539-546.

Fukazawa H, Hoshino K, Shiozawa T, Matsushita H, Terao Y. (2001) Identification and quantification of chlorinated bisphenol $A$ in wastewater from wastepaper recycling plants. Chemosphere 44, 973-979.

Fung EY, Ewoldsen NO, St Germain HA Jr, Marx DB, Miaw CL, Siew C (2000), Pharmacokinetics of bisphenol A released from a dental sealant. Journal of the American Dental Association 131, 51-58.

Gaffney, P. T., R. L. Buttenshaw, M. Ward, R. D. Diplock (1986). "Breast milk betaglucuronidase and neonatal jaundice." Lancet 1(8490): 1161-1162.

Galloway T, Cipelli R, Guralnik J, Ferrucci L, Bandinelli S, Corsi AM, Money C, McCormack P, Melzer D (2010). Daily bisphenol A excretion and associations with sex hormone concentrations: results from the InCHIANTI adult population study. Environmental Health Perspectives, 118, 1603-1608.

Gayrard V, Lacroix MZ, Collet SH, Viguié C, Bousquet-Melou A, Toutain PL, Picard-Hagen N. (2013) High bioavailability of bisphenol A from sublingual exposure. Environ Health Perspect. 121, 951-956.

Geens T, Aerts D, Berthot C, Bourguignon J-P., Goeyens L., Lecomte P., MaghuinRogister G., Pironnet A-M. Pussemier L., Scippo M-L., Van Loco J., Covaci A. (2012) A review of dietary and non-dietary exposure to bisphenol-A. Food and Chemical Toxicology 50, 3725-3740, 2012.

Geens T, Roosens L, Neels H and Adrian Covaci A (2009) Assessment of human exposure to Bisphenol-A, Triclosan and Tetrabromobisphenol-A through indoor dust intake in Belgium. Chemosphere 76: 755-760 
Gehring MJ. (2004) Verhalten der endokrin wirksamen Substanz Bisphenol A bei der kommunalen Abwasserentsorgung ,Dissertation, 2004 Technische Univ. Dresden.

Genuis, SJ, Beesoon S, Birkholz and Lobo RA (2011) Human Excretion of Bisphenol A: Blood, Urine and Sweat (BUS) Study. Journal of Environmental and Public Health, 2012: Article ID 185731, 1-10

Ginsberg G, Hattis D, Sonawane B, Russ A, Banati P, Kozlak M, Smolenski S, Golbe R, (2002). Evaluation of child/adult pharmacokinetic differences from a database derived from the therapeutic drug literature. Toxicological Sciences 66, 185-200.

Gioiosa L, Fissore E, Ghirardelli G, Parmigiani S, Palanza P (2007) Developmental exposure to low-dose estrogenic endocrine disruptors alters sex differences in exploration and emotional responses in mice. Horm Behav 52, 307-316.

Gioiosa L, Parmigiani S, Vom Saal FS, Palanza P. (2013) The effects of bisphenol A on emotional behavior depend upon the timing of exposure, age and gender in mice. Horm Behav. 63, 598-605.

Goodman JE, Witorsch RJ, McConnell EE, Sipes IG, Slayton TM, Yu CJ, Franz AM, Rhomberg LR. (2009) Weight-of-evidence evaluation of reproductive and developmental effects of low doses of bisphenol A. Crit Rev Toxicol. 39, 1-75.

Grazioso, C. F. and E. S. Buescher (1996). "Inhibition of neutrophil function by human milk." Cell Immunol 168: 125-132.

Gyllenhammer I, Tröger R, Glynn A, Rosen J, Hellenäs K-E, Lignell S. (2014) Serum levels of unconjugated bisphenol $A$ are below $0.2 \mathrm{ng} / \mathrm{ml}$ in Swedish nursing women when contamination is minimized. Environm Int. 64, 56-60.

Haighton LA, Hlywka J], Doull J, Kroes R, Lynch BS, Munro IC (2002). An evaluation of the possible carcinogenicity of bisphenol A to humans. Regul Toxicol Pharmacol. 35 (2 Pt 1), 238-254.

Haishima Y., Hayashi Y., Yagami T. and Nakamura A. (2001). Elution of Bisphenol-A from Hemodialysers consisting of polycarbonate and polysoulfone resins. Journal of Biomedical Materials Research 58 (2):209-215.

Hajszan T, Leranth C. (2010) Bisphenol A interferes with synaptic remodeling. Front Neuroendocrinol. 31, 519-530.

Han DH, Kim MJ, Jun EJ, Kim JB. (2012) Salivary bisphenol-A levels due to dental sealant/resin: a case-control study in Korean children. Journal of Korean Medical Science 2012;27: 1098-104.

Hanaoka T, Kawamura N, Hara K, Tsugane S. (2002) Urinary bisphenol A and plasma hormone concentrations in male workers exposed to bisphenol A diglycidyl ether and mixed organic solvents. Occupational and Environmental Medicine, 59, 625-628.

Hanioka N, Naito T and Narimatsu S, (2008). Human UDP-glucuronosyltransferase isoforms involved in bisphenol A glucuronidation. Chemosphere 74, 33-36.

Hanioka N, Oka H, Nagaoka K, Ikushiro S, Narimatsu S (2011) Effect of UDPglucuronosyltransferase 2B15 polymorphism on bisphenol A glucuronidation. Arch Toxicol 85: $1373-1381$.

Hao J, Wang J, Zhao W, Ding L, Gao E, Yuan W. (2011). Effect of bisphenol A exposure on sex hormone level in occupational women]. Wei Sheng Yan Jiu. 40, 312-4, 319.

Harthé C., Rinaldi S., Achaintre D., Rolland de Ravel M., Mappus E., Pugeat M., Dèchaud H. (2012) Bisphenol A-glucuronide measurement in urine samples Talanta, 100, 410413.

Hashimoto Y, Moriguchi Y, Oshima H, Kawaguchi M, Miyazaki K, Nakamura M (2001) Measurement of estrogenic activity of chemicals for the development of new dental polymers. Toxicol. In vitro $15,421-425$. 
Hashimoto Y, Nakamura M. (2000) Estrogenic activity of dental materials and bisphenol A related chemicals in vitro. Dent. Mater. J. 19, 245-262.

He Z, Paule MG, Ferguson SA (2012). Low oral doses of bisphenol A increase volume of the sexually dimorphic nucleus of the preoptic area in male, but not female, rats at postnatal day 21. Neurotoxicology and Teratology, 34, 331-337.

Helander A, Dahl H. (2005) Urinary tract infection: a risk factor for false-negative urinary ethyl glucuronide but not ethyl sulfate in the detection of recent alcohol consumption. Clin Chem. 51, 1728-1730.

Hengstler JG, Foth $H$, Gebel T, Kramer PJ, Lilienblum W, Schweinfurth $H$, Völkel W, Wollin KM, Gundert-Remy U. (2011) Critical evaluation of key evidence on the human health hazards of exposure to bisphenol A. Crit Rev Toxicol. 41, 263-291.

Hiroi H, Tsutsumi O, Takeuchi T, Momoeda M, Ikezuki Y, Okamura A, Yokota H, Taketani Y. (2004). Differences in serum bisphenol A concentrations in premenopausal normal women and women with endometrial hyperplasia. Endocrine Journal, 51, 595-600.

Ho SM, Tang WY, Belmonte J, Prins GS (2006) Developmental exposure estradiol and bisphenol A (BPA) increases susceptibility to prostate carcinogenesis and epigenetically regulates phosphodiesterase type 4 variant (PDE4D4) in the rat prostate Cancer Res, 66, 5624-5632.

Hoekstra EJ and Simoneau C. (2013) Release of bisphenol A from polycarbonate - A review. Critical Reviews in Food Science and Nutrition 53, 386-402.

Honma S, Suzuki A, Buchanan DL, Katsu Y, Watanabe H, Iguchi T. (2002) Low dose effect of in utero exposure to bisphenol $A$ and diethylstilbestrol on female mouse reproduction. Reprod Toxicol, 16, 117-122.

Huc L, Lemarie A, Gueraud F, Helies-Toussaint C (2012). Low concentrations of bisphenol $A$ induce lipid accumulation mediated by the production of reactive oxygen species in the mitochondria of HepG2 cells. Toxicology in vitro, 26, 709-717.

Hugo E.R., Brandebourg T.D., Woo J.G., Loftus J., Alexander J.W., Ben-Jonathan N.(2008). Bisphenol A at environmentally relevant doses inhibits adiponectin release from human adipose tissue explants and adipocytes. Environmental Health Perspectives $116,1642-1647$.

Hunt PA, Koehler KE, Susiarjo M, Hodges CA, Ilagan A, Voigt RC, Thomas S, Thomas BF, Hassold TJ. (2003) Bisphenol a exposure causes meiotic aneuploidy in the female mouse. Curr Biol. 13, 546-553.

Ichihara T, Yoshino $\mathrm{H}$, Imai N, Tsutsumi T, Kawabe M, Tamano S, Inaguma S, Suzuki S, Shirai T. (2003) Lack of carcinogenic risk in the prostate with transplacental and lactational exposure to bisphenol A in rats. J Toxicol Sci. 28, 165-171.

Ike M, Chen MY, Danzl E, Sei K, Fujita M. (2006) Biodegradation of a variety of bisphenols under aerobic and anaerobic conditions. Water Sci. Technol. 53, 153-159.

Inagaki T, Frankfurt $M$ and Luine V. (2012) Estrogen-induced memory enhancements are blocked by acute bisphenol $A$ in adult female rats: role of dendritic spines. Endocrinology, 153, 3357-3367.

Indumathi D, Jayashree S, Selvaraj J, Sathish S, Mayilvanan C, Akilavalli N and Balasubramanian K. (2013). Effect of bisphenol-A on insulin signal transduction and glucose oxidation in skeletal muscle of adult male albino rat. Human \& Experimental Toxicology, 32, 960-971.

Inoue K, Yamaguchi A, Wada M, Yoshimura Y, Makino T, Nakazaw H. (2001) Quantitative detection of bisphenol $A$ and bisphenol A diglycidyl ether metabolites in human plasma by liquid chromatography-electrospray mass spectrometry. J Chromatogr B Biomed Sci Appl. 765, 121-126. 
Inoue K, Wada M, Higuchi T, Osgio S, Umeda T, Yoshimura Y, Nahazawa H (2002) Application of liquid chromatography-mass spectrometry to the quantification of bisphenaol A in humen semen. J Chromatogr B Analyt Technol Biomed Life Sci 773, 97 102.

Inoue K, Kawaguchi M, Funakoshi Y, Nakazawa H. (2003) Size-exclusion flow extraction of bisphenol $A$ in human urine for liquid chromatography-mass spectrometry. J Chromatogr B Analyt Technol Biomed Life Sci. 798, 17-23.

IPCS (2005) Chemical-specific adjustment factors for interspecies differences and human variability: Guidance document for use of data in dose/concentration-response assessment World Health Organization, Geneva, Switzerland.

Ishido M, Masuo Y, Kunimoto M, Oka S, Morita M. (2004). Bisphenol A causes hyperactivity in the rat concomitantly with impairment of tyrosine hydroxylase immunoreactivity. J Neurosci Res 76: 423-433.

Ishido M, Masuo Y, Terasaki M, Morita M. (2011). Rat Hyperactivity by Bisphenol A, but Not by Its Derivatives, 3-hydroxybisphenol A or Bisphenol A 3,4-quinone. Toxicology Letters 206: 300-305.

Iso T, Watanabe T, Iwamoto T, Shimamoto A, Furuichi Y.(2006) DNA damage caused by bisphenol A and estradiol through estrogenic activity. Biol Pharm Bull. 29, 206-210.

Itoh H, Iwasaki M, Hanaoka T, Sasaki H, Tanaka T, Tsugane S. (2007). Urinary bisphenol-A concentration in infertile Japanese women and its association with endometriosis: a cross-sectional study. Environmental Health and Preventive Medicine, $12,258-264$.

Izzotti A, Kanitz S, D'Agostini F, Camoirano A, De Flora S. (2009) Formation of adducts by bisphenol A, an endocrine disruptor, in DNA in vitro and in liver and mammary tissue of mice. Mutat Res. 679, 28-32.

Jacobi U, Kaiser M, Toll R, Mangelsdorf S, Audring H, Otberg N, Sterry W, Lademann J (2007). Porcine ear skin: an in vitro model for human skin. Skin Res. Technol. 13, 1924.

Jana SK, Okamoto T, Kugita T, Namba S. (2005) Selective synthesis of bisphenol F catalysed by microporous H-beta zeolite. Appl. Catal. A 288, 80-85.

Jasarevic E, Geary DC and Rosenfeld CS (2012). Sexually selected traits: a fundamental framework for studies on behavioral epigenetics. ILAR Journal, 53, 253-269.

Jayashree S, Indumathi D, Akilavalli N, Sathish S, Selvaraj J and Balasubramanian K, (2013). Effect of Bisphenol-A on insulin signal transduction and glucose oxidation in liver of adult male albino rat. Environmental Toxicology and Pharmacology, 35, 300-310

Jenkins S, Raghuraman N, Eltoum I, Carpenter M, Russo J, Lamartiniere CA (2009) Oral exposure to bisphenol a increases dimethylbenzanthracene-induced mammary cancer in rats. Environ Health Perspect. 117, 910-915.

Jenkins S, Wang J, Eltoum I, Desmond R and Lamartiniere CA, (2011). Chronic oral exposure to bisphenol $A$ results in a nonmonotonic dose response in mammary carcinogenesis and metastasis in MMTV-erbB2 mice. Environmental Health Perspectives, 119, 1604-1609.

Jie H, Ke H, Qing Z, Lei C., Yongqiang W, Zibin Z (2006). Study on depolymerization of polycarbonate in supercritical ethanol. Polym. Degrad.Stabil. 91:2307-2314.

Jiménez-Díaz I, Zafra-Gómez A, Ballesteros O, Navea N, Navalón A, Fernández MF, Olea $\mathrm{N}$, Vílchez JL. (2010) Determination of Bisphenol A and its chlorinated derivatives in placental tissue samples by liquid chromatography-tandem mass spectrometry. J Chromatogr B Analyt Technol Biomed Life Sci. 878, 3363-3369. 
Johnson GE, Parry EM. (2008). Mechanistic investigations of low dose exposures to the genotoxic compounds bisphenol-A and rotenone. Mutat Res. 651, 56-63.

Jones LP, Sampson A, Kang HJ, Kim HJ, Yi YW, Kwon SY, Babus JK, Wang A, Bae I. (2010) Loss of BRCA1 leads to an increased sensitivity to Bisphenol A. Toxicol Lett. 199, 261-268.

Jones BA, Shimell JJ and Watson NV (2011). Pre- and postnatal bisphenol A treatment results in persistent deficits in the sexual behavior of male rats, but not female rats, in adulthood. Hormones and Behavior, 59, 246-251.

Jones BA and Watson NV, (2012). Perinatal BPA exposure demasculinizes males in measures of affect but has no effect on water maze learning in adulthood. Hormones and Behavior, 61, 605-610.

Joskow R, Barr DB, Barr JR, Calafat AM, Needham LL, Rubin C. (2006) Exposure to bisphenol A from bis-glycidyl dimethacrylate-based dental sealants. Journal of the American Dental Association 2006; 137: 353-62.

Kabsy Y, Baudin G, Vinti H, Novellas S, Mannone L, Chevallier P, Mounier N (2010) Peripherally inserted central catheters in onco-hematology - Bull Cancer. 97, 1067-1071.

Kaddar N, Bendridi N, Harthé C, de Ravel MR, Bienvenu AL, Cuilleron CY, Mappus E, Pugeat M, Déchaud H. (2009) Development of a radioimmunoassay fort eh measurement of bisphenol A in biological samples. Anal Chim Acta. 645, 1-4, 2009.

Kaddar N, Harthe C, Déchaud H, Mappus E, Pugeat M (2008). Cutaneous penetration of bi $\neg$ sphenol A in pig skin. J Toxicol Environ Health (A) 71, 471-473.

Kang $\mathrm{JH}$, Kondo $\mathrm{F}$ and Katayama $\mathrm{Y}$ (2006) Human exposure to bisphenol A. Toxicology 226, 79-89.

Kang Y G, Kim J Y, Kim J, Won P J, Nam J H (2011) Release of bisphenol A from resin composite used to bond orthodontic lingual retainers. Am J Orthod Dentofacial Orthop. 6, 779-789.

Kasper-Sonnenberg M, Wittsiepe J, Koch HM, Fromme H and Wilhelm M, (2012) Determination of bisphenol $a$ in urine from mother-child pairs-results from the duisburg birth cohort study, Germany. J Toxicol Environ Health A, 75, 429-437.

Kass L, Altamirano GA, Bosquiazzo VL, Luque EH and Munoz-de-Toro M. (2012) Perinatal exposure to xenoestrogens impairs mammary gland differentiation and modifies milk composition in Wistar rats. Reproductive Toxicology, 33, 390-400.

Katoh K, Matsuda A, Ishigami A, Yonekura S, Ishiwata H, Chen C, Obara Y. (2004) Suppressing effects of bisphenol $A$ on the secretory function of ovine anterior pituitary cells. Cell Biol Int. 28, 463-469.

KEMI (2011) Bisfenol A - Rapport från ett regeringsuppdra. Rapport 2/11

Kendziorski JA, Kendig EL, Gear RB, Belcher SM (2012) Strain specific induction of pyometra and differences in immune responsiveness in mice exposed to 17a-ethinyl estradiol or the endocrine disrupting chemical bisphenol A. Reprod Toxicol. 34, 22-30

Kietzmann M, Kranke P, Moder M, Schrader S, Wahren M (1999). Application of deuterated compounds for investigations of percutaneous absorption of chemical substances. Isot. Environ. Health Stud. 35, 127-134.

Kiguchi M, Fujita S, Lee J, Shimizu N, Koshikawa N. (2007). Behavioral responses to methylphenidate and apomorphine in rats exposed neonatally to bisphenol-A. J Oral Sci 49: 311-318.

Kiguchi M, Fujita S, Oki H, Shimizu N, Cools AR, Koshikawa N. (2008). Behavioural characterisation of rats exposed neonatally to bisphenol-A: responses to a novel environment and to methylphenidate challenge in a putative model of attention-deficit hyperactivity disorder. J Neural Transm 115: 1079-1085. 
Kim YH, Kim CS, Park S, Han SY, Pyo MY, Yang M. (2003). Gender differences in the levels of bisphenol A metabolites in urine. Biochem Biophys Res Commun 312:441-448.

Kim MR, Kim HS, Park DW, Lee JK. (2001) Synthesis of cyclic carbonates based on diglycidyl ether of bisphenol $\mathrm{S}$ by quaternary ammonium salts. React. Kinet. Catal. Lett. 72, 373-381.

Kim K, Park H, Yang $W$ and Lee JH. (2011) Urinary concentrations of bisphenol A and triclosan and associations with demographic factors in the Korean population. Environmental Research, 111, 1280-1285.

Kim ME, Park HR, Gong EJ, Choi SY, Kim HS and Lee J. (2011) Exposure to bisphenol A appears to impair hippocampal neurogenesis and spatial learning and memory. Food and chemical toxicology, 49, 3383-3389.

Kim Y-J, Yun H-J and Ryu J-C. (2011) Expression profiling of estrogen responsive genes on bisphenol A, 4-nonylphenol and $17 \beta$-estradiol treatment using in house cDNA microarray. BioChip Journal, 5, 86-94.

Kingman A, Hyman J, Masten, SA, Jayaranm B, Smith C, Eicmiller F, Arnold MC, Wong PA, Schaeffer JM, Solanki S, Dunn WJ. (2012) Bisphenol A and other compounds in human saliva and urine associated with the placement of composite restorations. J Am Dent Assoc 143,1292-1302.

Kitamura S, Jinno N, Ohta S, Kuroki H, Fujimoto N. (2002) Thyroid hormonal activity of the flame retardants tetrabromobisphenol $A$ and tetrachlorobisphenol $A$. Biochem. Biophys. Res. Commun. 293, 554-559.

Kitamura S, Suzuki T, Sanoh S, Kohta R, Jinno N, Sugihara K, Yoshihara S, Fujimoto N, Watanabe H, Ohta S. (2005) Comparative study of the endocrine-disrupting activity of bisphenol A and 19 related compounds. Toxicol. Sci. 84, 249-259.

Kliegman RM, Stanton BF, St Geme III JW, Schor NF, Behrman RE (Editors), Nelson Textbook of Paediatrics. 19th Edition. 2011 Chapter 91 The high risk infant. R. M.. Elsevier Saunders, Philadelphia, PA, USA, 2011.

Kloukos D, Pandis N, Eliades T. (2013) Bisphenol-A and residual monomer leaching from orthodontic adhesive resins and polycarbonate brackets: a systematic review. Am J Orthod Dentofacial Orthop. 2013 Apr; 143(4 Suppl):S104-12.e1-2. doi: 10.1016/j.ajodo.2012.11.015

Kobayashi K, Miyagawa M, Wang RS, Sekiguchi S, Suda M, Honma T. (2002) Effects of in utero and lactational exposure to bisphenol $A$ on somatic growth and anogenital distance in $\mathrm{F} 1$ rat offspring. Ind Health. 40, 375-381.

Kobayashi K, Ohtani K, Kubota H, Miyagawa M. (2010) Dietary exposure to low doses of bisphenol $A$ : effects on reproduction and development in two generations of C57BL/6] mice. Congenit Anom (Kyoto). 50, 159-170.

Koch HM, Kolossa-Gehring M, Schroter-Kermani C, Angerer J and Bruning T. (2012). Bisphenol $\mathrm{A}$ in $24 \mathrm{~h}$ urine and plasma samples of the German Environmental Specimen Bank from 1995 to 2009: A retrospective exposure evaluation. J Expo Sci Environ Epidemiol, 22, 610-616.

Komada M, Asai Y, Morii M, Matsuki M, Sato M and Nagao T. (2012). Maternal bisphenol $A$ oral dosing relates to the acceleration of neurogenesis in the developing neocortex of mouse fetuses. Toxicology, 295, 31-38.

Kosarac I, Kubwabo C, Lalonde K, Foster W (2012) A novel method for the quantitative determination of free and conjugated bisphenol $\mathrm{A}$ in human maternal and umbilical cord blood serum using a two-step solid phase extraction and gas chromatography/tandem mass spectrometry Journal of Chromatography B, 898, 90- 94, 2012.

Krieter D H, Fischer R, Lemke H-D, Canaud B, Wanner C (2009). Bisphenol A (BPA) as an Uremic Toxin: Large Differences in Elution from Dialyzers and Elevated Plasma Levels in 
Maintenance Dialysis Patients. Poster presentation at the EDTA (European Dialysis and Transplant Association) 2009, Milan.

Krieter DH, Canaud B, Lemke HD, Rodriguez A, Morgenroth PA, von Appen K, Dragoun GP and Wanner C (2013). Bisphenol A in Chronic Kidney Disease. Artif Organs. 37, 283290.

Kundakovic M, Gudsnuk K, Franks B, Madrid J, Miller RL, Perera FP, Champagne FA. (2013) Sex-specific epigenetic disruption and behavioral changes following low-dose in utero bisphenol A exposure. Proc Natl Acad Sci U S A. 110, 9956-9961.

Kurebayashi $H$, Betsui $H$, Ohno Y. (2003). Disposition of a low dose of 14C-bisphenol A in male rats and its main biliary excretion as BPA glucuronide. Toxicol Sci 73:17-25.

Kurebayashi H, Harada R, Stewart RK, Numata H, Ohno Y. (2002). Disposition of a low dose of bisphenol A in male and female cynomolgus monkeys. Toxicol Sci 68:32-42.

Kurebayashi H, Okudaira K, Ohno Y (2010). Species difference of metabolic clearance of bisphenol A using cryopreserved hepatocytes from rats, monkeys and humans. Toxicol Lett 198:210-215.

Kuruto-Niwa R, Nozawa R, Miyakoshi T, Shiozawa T, Terao Y. (2005) Estrogenic activity of alkylphenols, bisphenol $\mathrm{S}$, and their chlorinated derivatives using a GFP expression system. Environ. Toxicol. Pharmacol. 19, 121-130.

Kuruto-Niwa R, Tateoka Y, Usuki Y, Nozawa R (2007). Measurement of bisphenol A concentrations in human colostrum. Chemosphere 66, 1160-1164.

Kwon S, Stedman DB, Elswick BA, Cattley RC, Welsch F. (2000). Pubertal development and reproductive functions of $\mathrm{Crl}: \mathrm{CD}$ BR Sprague-Dawley rats exposed to bisphenol $\mathrm{A}$ during prenatal and postnatal development. Toxicol Sci. 55, 399-406.

Lacroix M Z, Puel S, Collet S H, Corbel T, Picard- Hagen N, Toutain PL, Viguié C, Gayrard $V$ (2011) Simultaneous quantification of bisphenol A and its glucuronide metabolite (BPA$\mathrm{G})$ in plasma and urine: Applicability to toxicokinetic investigations Talanta 85, 20532059, 2011.

LaKind J S, Goodman M, Naiman D Q (2012), Use of NHANES data to link chemical exposures to chronic diseases: a cautionary tale. PLoS One, 7 (12): p. e51086.

Lakind JS, Naiman DQ. (2008) Lakind JS, Naiman DQ. (2008). Bisphenol A (BPA) daily intakes in the United States: estimates from the 2003-2004 NHANES urinary BPA data. J Expo Sci Environ Epidemiol. 18, 608-615

Lakind JS, Naiman DQ. (2010). Daily intake of bisphenol A and potential sources of exposure: 2005-2006 National Health and Nutrition Examination Survey. J Expo Sci Environ Epidemiol advance online publication 17 Mar 2010; doi:10.1038/jes.2010.9

Lang I A, Galloway T S, Scarlett A, Henley W E, Depledge M, Wallace R B, Melzer D (2008) Association of urinary bisphenol A concentration with medical disorders and laboratory abnormalities in adults. JAMA 300, 1303-1310.

Lee C, Prins GS, Henneberry MO, Grayhack JT (1981) Effect of estradiol on the rat prostate in the presence and absence of testosterone and pituitary. J Andro 2:293-299)

Lee J, Lee SJ, Lim KT. (2012) CTB glycoprotein (75kDa) inhibits IgE releasing, TNF-a and IL-6 expressed by bisphenol A in vivo and in vitro. Food Chem Toxicol. 50, 2109-2117

Lee YJ, Ryu HY, Kim HK, Min CS, Lee JH, Kim E, Nam BH, Park JH, Jung JY, Jang DD, Park EY, Lee KH, Ma JY, Won HS, Im MW, Leem JH, Hong YC, Yoon HS. (2008). Maternal and fetal exposure to bisphenol A in Korea. Reprod Toxicol 25, 413-419.

Lewis JB, Rueggeberg FA, Lapp CA, Ergle JW. (1999) Identification and charakterisation of estrogen-like components in commercial resin-based dental restorative materials. Clin Oral Investig 3, $107-113$. 
Li DK, Zhou Z, Miao M, He Y, Qing D, Wu T, Wang J, Weng X, Ferber J, Herrinton LJ, Zhu Q, Gao E, Yuan W. (2010a). Relationship between urine bisphenol-A (BPA) level and declining male sexual function. Journal of Andrology, 31, 500-506.

Li DK, Zhou Z, Miao M, He Y, Wang J, Ferber J, Herrinton LJ, Gao E, Yuan W. (2010b). Urine bisphenol-A (BPA) level in relation to semen quality. Fertility and Sterility, 95, 625630.

Li DK, Zhou Z, Qing D, He Y, Wu T, Miao M, Wang J, Weng X, Ferber JR, Herrinton LJ, Zhu Q, Gao E, Checkoway H, Yuan W. (2010c). Occupational exposure to bisphenol-A (BPA) and the risk of self-reported male sexual dysfunction. Human Reproduction (Oxford, England), 25, 519-527.

Liao C, Kannan K. (2012) Determination of free and conjugated forms of bisphenol A in human urine and serum by liquid chromatography-tandem mass spectrometry. Environ Sci Technol. 2012 May 1;46(9):5003-9. doi: 10.1021/es300115a. Epub 2012 Apr 19.

Lin Y, Sun X, Qiu L, Wei J, Huang Q, Fang C, Ye T, Kang M, Shen H and Dong S. (2013). Exposure to bisphenol $A$ induces dysfunction of insulin secretion and apoptosis through the damage of mitochondria in rat insulinoma (INS-1) cells. Cell Death Dis, 4, e460.

Liu Z, Wolff MS, Moline J. (2005). Analysis of environmental biomarkers in urine using an electrochemical detector. J Chromatogr B Analyt Technol Biomed Life Sci. 819, 155-159.

Loganathan SN, Kannan K. (2011). Occurrence of Bisphenol A in Indoor Dust from Two Locations in the Eastern United States and Implications for Human Exposures. Arch Environ Contam Toxicol 61, 68-73.

Lopez-Cervantes J. and Paseiro-Losada P., (2003). Determination of bisphenol A in, and its migration from, PVC stretch film used for food packaging. Food Additives \& Contaminants, 20, 596-606.

Lopez-Espinosa MJ, Granada A, Araque P, Molina JM, Puertollano MC, Rivas A, Fernández MF, Cerrillo I, Olea-Serrano MF, López C, Olea N.(2007) Oestrogenicity of paper and cardboard extracts used as food containers. Food Additives and Contaminants, 24, 95102.

Lucas JN, Rudmann DG, Credille KM, Irizarry AR, Peter A, Snyder PW (2007) The rat mammary gland: morphologic chamges as an indicator of systemic hormonal pertubations induced by xenobiotiocs. Toxiol Pathol 35, 199-207.

Mackay H, Patterson ZR, Khazall R, Patel S, Tsirlin D and Abizaid A. (2013) Organizational Effects of Perinatal Exposure to Bisphenol-A and Diethylstilbestrol on Arcuate Nucleus Circuitry Controlling Food Intake and Energy Expenditure in Male and Female CD-1 Mice. Endocrinology, 154, 1465-1475.

Maia J., Cruz J.M., Sendón R., Bustos J. et al. (2010). Effect of amines in the release of bisphenol A from polycarbonate baby bottles. Food Research International 43:12831288, 2010.

Manabe A, Kaneko S, Numazawa S, Itoh K, Inoue M, Hisamitsu H, Sasa R, Yoshida T. (2000) Detection of bisphenol-A in dental materials by gas chromatography-mass spectrometry. Dent Mater J. 19, 75-86.

Markham DA, Waechter JM Jr, Wimber M, Rao N, Connolly P, Chuang, JC, Hentges S, Shiotsuka RN, Dimond S, Chappelle AH. (2010). Development of a method for the determination of bisphenol $A$ at trace concentrations in human blood and urine and elucidation of factors influencing method accuracy and sensitivity. J Anal Toxicol 34:293303.

Marmugi A, Ducheix S, Lasserre F, Polizzi A, Paris A, Priymenko N, Bertrand-Michel J, Pineau T, Guillou H, Martin PG and Mselli-Lakhal L. (2012) Low doses of bisphenol A induce gene expression related to lipid synthesis and trigger triglyceride accumulation in adult mouse liver. Hepatology, 55, 395-407. 
Marquet F., Payan J-P., Beydon D., Wathier L., Grandclaude M-C., Ferrari E. (2011). In vivo and ex vivo percutaneous absorption of [14C]-bisphenol $A$ in rats: a possible extrapolation to human absorption? Arch Toxicol 85, 1035-1043.

Maserejian NN, Trachtenberg FL, Hauser R, McKinlay S, Shrader P, Tavares M, Bellinger DC. (2012a) Dental composite restorations and Psychosocial function in children. Pediatrics. 130, e328-338.

Masuda S, Terashima Y, Sano A, Kuruto R, Sugiyama Y, Shimoi K, Tanji K, Yoshioka H, Terao Y, Kinae N (2005) Changes in the mutagenic and estrogenic activities of bisphenol A upon treatment with nitrite. Mutat Res. 585, 137-146.

Masuo Y, Ishido M, Morita M, Oka S. (2004). Effects of neonatal treatment with 6hydroxydopamine and endocrine disruptors on motor activity and gene expression in rats. Neural Plast 11: 59-76.

Matsuda S, Matsuzawa D, Ishii D, Tomizawa H, Sutoh C, Nakazawa K, Amano K, Sajiki J, Shimizu E. (2012). Effects of perinatal exposure to low dose of bisphenol A on anxiety like behavior and dopamine metabolites in brain. Prog Neuropsychopharmacol Biol Psychiatry. 39, 273-279.

Matsumoto J, Yakota $\mathrm{H}$ and Yuasa A. (2002). Developmental increases in rat hepatic microsomalUDP-glucuronosyltransferase activities toward xenoestrogens and decreases during pregnancy. Environmental Health Perspectives 110, 193-196

Matsumoto $H$, Adachi $S$ and Suzuki $Y$ (2005) Bisphenol A in ambient air particulates responsible for the proliferation of MCF-7 human breast cancer cells and its concentration changes over 6 months. Arch Environ Contam Toxicol 48: 459-466.

Mazur Christopher S., John F. Kenneke, Janet K. Hess-Wilson, and John C. (2010) LiPSUPScomb Differences between Human and Rat Intestinal and Hepatic Bisphenol A Glucuronidation and the Influence of Alamethicin on In vitro Kinetic Measurements Drug Metabolism and Disposition 38:2232-2238, 2010.

Mazzaoui SA, Burrow MF, Tyas MJ, Rooney FR, Capon RJ. (2002) Long-term quantification of the release of monomers from dental resin composites and a resinmodified glass ionomer cement. J Biomed Mater Res. 63, 299-305.

McKeen LW (2012): The effect of sterilization methods on plastics abs elastomers, 3rd Edition Thechnology \& Enginiiering Elsevier ed. 2012 pp 355.

Meeker JD, Calafat AM, Hauser R. (2010a) Urinary bisphenol A concentrations in relation to serum thyroid and reproductive hormone levels in men from an infertility clinic. Environ Sci Technol. 44, 1458-1463.

Meeker J D, Ehrlich S, Toth T L, Wright D L, Calafat A M, Trisini A T, Ye X, Hauser R (2010b) Semen quality and sperm DNA damage in relation to urinary bisphenol $A$ among men from an infertility clinic. Reproductive Toxicology 30, 532-539.

Melzer D, Gates P, Osborn NJ, Henley WE, Cipelli R, Young A, Money C, McCormack P, Schofield P, Mosedale D, Grainger D and Galloway TS. (2012) Urinary bisphenol a concentration and angiography-defined coronary artery stenosis. PLoS One, 7, e43378.

Melzer D, Harries L, Cipelli R, Henley W, Money C, McCormack P, Young A, Guralnik J, Ferrucci L, Bandinelli S, Corsi AM and Galloway T. (2011) Bisphenol A exposure is associated with in vivo estrogenic gene expression in adults. Environmental Health Perspectives, 119, 1788-1793.

Melzer D, Osborne NJ, Henley WE, Cipelli R, Young A, Money C, McCormack P, Luben R, Khaw KT, Wareham NJ and Galloway TS. (2012) Urinary bisphenol A concentration and risk of future coronary artery disease in apparently healthy men and women. Circulation, $125,1482-1490$. 
Melzer D, Rice N E, Lewis C, Henley W E, Galloway T S (2010) Association of urinary bisphenol A concentration with heart disease: evidence from NHANES 2003/06. PloS One 5, e8673.

Mendes GC, Branddo TR, Silva CL (2007) Ethylene oxide sterilization of medical devices/ A review. Am J Infect Control. 35, 574-581.

Mendiola J, Jørgensen N, Andersson AM, Calafat AM, Ye X, Redmon JB, Drobnis EZ, Wang C, Sparks A, Thurston SW, Liu F, Swan SH. (2010). Are environmental levels of bisphenol A associated with reproductive function in fertile men? Environmental Health Perspectives, 118, 1286-1291.

Mercea P. (2009). Physicochemical Processes Involved in Migration of Bisphenol A from Polycarbonate. Journal of Applied Polymer Science 112 (2): 579-593.

Miao M, Yuan W, Zhu G, He X, Li DK. (2011) In utero exposure to bisphenol-A and its effect on birth weight of offspring. Reprod Toxicol, 32, 64-68.

Mielke H, Gundert-Remy U. (2012) Physiologically based toxicokinetic modelling as a tool to support risk assessment: three case studies. J Toxicol. 2012; 2012:359471

Mielke, H. Partosch F., and Gundert-Remy U.(2011) The contribution of dermal exposure to the internal exposure of bisphenol A in man Toxicol Lett. 28, 190-198.

Mielke, H., Gundert-Remy, U. (2009) Bisphenol A levels in blood depend on age and exposure Tox. Lett. 190, 32-40.

Mita L, Baldi A, Diano N, Viggiano E, Portaccio M, Nicolucci C, Grumiro L, Menale C, Mita DG, Spugnini EP, Viceconte R, Citro G, Pierantoni R, Sica V, Marino M, Signorile PG and Bianco M, (2012). Differential accumulation of BPA in some tissues of offspring of Balb-C mice exposed to different BPA doses. Environmental Toxicology and Pharmacology, 33, 9-15

Miyawaki J, Sakayama K, Kato H, Yamamoto H, Masuno H (2007) Perinatal and postnatal exposure to bisphenol $A$ increases adipose tissue mass and serum cholesterol level in mice. Journal of Atherosclerosis and Thrombosis, 14, 245-252.

Mok-Lin E, Ehrlich S, Williams P L, Petrozza J, Wright D L, Calafat A M, Ye X, Hauser R (2010) Urinary bisphenol A concentrations and ovarian response among women undergoing IVF. International Journal of Andrology 33, 385-393.

Molina-Molina JM, Amaya E, Grimaldi M, Sáenz JM, Real M, Fernández MF, Balaguer $P$, Olea N. (2013). In vitro study on the agonistic and antagonistic activities of bisphenol-S and other bisphenol-A congeners and derivatives via nuclear receptors. Toxicol. Appl. Pharmacol. 272, 127-136.

Moral R, Wang R, Russo IH, Lamartiniere CA, Pereira J, Russo J. (2008) Effect of prenatal exposure to the endocrine disruptor bisphenol $A$ on mammary gland morphology and gene expression signature. J Endocrinol. 196, 101-112.

Mørck T J, Sorda G, Bechi, N, Rasmussen B S, Nielsen, J B, Ietta F, Rytting E, Mathiesen, L, Paulesu L, Knudsen L E (2010). Placental transport and in vitro effects of Bisphenol A. Reprod. Toxicol. 30, 131-137.

Morrissey RE, George JD, Price CJ, Tyl RW, Marr MC, Kimmel CA. (1987). The developmental toxicity of bisphenol $A$ in rats and mice. Fundam Appl Toxicol. 8, 571-582.

Mose T, Mathiesen L, Karttunen V, Nielsen JKS, Sieppi E, Kummu M, Mørck TA, Myöhänen K, Partanen H, Vähäkangas K, Knudsen LE and Myllynen P, (2012). Meta-analysis of data from human ex vivo placental perfusion studies on genotoxic and immunotoxic agents within the integrated European project NewGeneris. Placenta, 33, 433-439.

Murakami K, Ohashi A, Hori H, Hibiya M (2007). Accumulation of bisphenol a in hemodialysis patients. Blood Purif 25(3): 290-294. 
Murray TJ, Maffini MV, Ucci AA, Sonnenschein C, Soto AM.(2007). Induction of mammary gland ductal hyperplasias and carcinoma in situ following fetal bisphenol A exposure. Reprod Toxicol. 23, 383-390.

Nachman RM, Fox SD, Golden WC, Sibinga E, Veenstra TD, Groopman JD and Lees PSJ, (2013). Urinary Free Bisphenol A and Bisphenol A-Glucuronide Concentrations inNewborns. The Journal of Pediatrics, http://dx.doi.org/10.1016/j.jpeds.2012.11.083

Nadal A. (2013) Obesity: Fat from plastics? Linking bisphenol A exposure and obesity. Nat Rev Endocrinol. 9, 9-10.

Nagel SC, Vom Saal Frederick S, Thayer Kristina AI, Dhar Minati G, Boechler Michael, Welshons Wade V. (1997) Relative Binding Affinity-Serum Modified Access (RBA-SMA) Assay Predicts the Relative In Vivo Bioactivity of the Xenoestrogens Bisphenol $A$ and Octylphenol, Environmental Health Perspectives, 105, 70-76.

Nahar MS, Kim JH, Sartor MA, Dolinoy DC. (2013) Bisphenol A-associated alterations in the expression and epigenetic regulation of genes encoding xenobiotic metabolizing enzymes in human fetal liver. Environ Mol Mutagen. doi: 10.1002/em.21823. [Epub ahead of print]

Naik P, Vijayalaxmi KK. (2009). Cytogenetic evaluation for genotoxicity of bisphenol-A in bone marrow cells of Swiss albino mice. Mutat Res. 676, 106-112.

Nakajima Y, Goldblum RM, Midoro-Horiuti T (2012) Fetal exposure to bisphenol A as a risk factor for the development of childhood asthma: an animal model study. Environ Health. 11:8

Nakamura S, Tezuka Y, Ushiyama A, Kawashima C, Kitagawara Y, Takahashi K, Ohta S and Mashino T. (2011) Ipso substitution of bisphenol A catalyzed by microsomal cytochrome P450 and enhancement of estrogenic activity. Toxicology Letters, 203, 9295.

Nanjappa MK, Simon L, Akingbemi BT (2012). The industrial chemical bisphenol A (BPA) interferes with proliferative activity and development of steroidogenic capacity in rat Leydig cells. Biol Reprod. 86, 135, 1-12.

Nishiyama T, Ogura K, Nakano H, Kaku T, Takahashi E, Ohkubo Y, Sekine, K, Hiratsuka A, Kadota S and Watabe T, (2002). Sulfation of Environmental Estrogens by Cytosolic Human Sulfotransferases. Drug Metabolism and Pharmacokinetics 17, 221-228.

Niwa T, Fujimoto M, Kishimoto K, Yabusaki Y, Ishibashi F, Katagiri M (2001).Metabolism and interaction of bisphenol $\mathrm{A}$ in human hepatic cytochrome P450 and steroidogenic CYP17. Biol. Pharm. Bull. 24, 1064-1067.

Nunez AA, Kannan K, Giesy JP, Fang J, Clemens LG. (2001). Effects of bisphenol A on energy balance and accumulation in brown adipose tissue in rats. Chemosphere 42(8):917-922.

Okuda K, Takiguchi M, Yoshihara S. (2010). In vivo estrogenic potential of 4-methyl2,4-bis(4-hydroxyphenyl)pent-1-ene, an active metabolite of bisphenol A, inuterus of ovariectomized rat. Toxicol. Lett. 197, 7-11.

Okuda K, Fukuuchi T, Takiguchi M, Yoshihara S (2011) Novel Pathway of Metabolic Activation of Bisphenol A-Related Compounds for Estrogenic Activity Drug Metabolism and Disposition 39:1696-1703, 2011

Olea N, Pulgar R, Pérez P, Olea-Serrano F, Rivas A, Novillo-Fertrell A et al. (1996) Estrogenicity of resin-based composites and sealants used in dentistry. Environmental Health Perspectives 1996; 104: 298-305.

Otaka H, Yasuhara A, Morita M (2003). "Determination of bisphenol A and 4-nonylphenol in human milk using alkaline digestion and cleanup by solid-phase extraction." Analytical Sciences 19, 1663-1666. 
Pacchierotti F, Ranaldi R, Eichenlaub-Ritter U, Attia S, Adler ID. (2008). Evaluation of aneugenic effects of bisphenol A in somatic and germ cells of the mouse. Mutat Res. 651, 64-70.

Pacifici G M, Kubrich M, Giuliani L, de Vries M, Rane A (1993). Sulphation and glucuronidation of ritodrine in human foetal and adult tissues. Eur. J. Clin. Pharmacol. 44, 259-264.

Padmanabhan V, Siefert K, Ransom S, Johnson T, Pinkerton J, Anderson L, Tao L, Kannan K. (2008) Maternal bisphenol-A levels at delivery: a looming problem? J Perinatol 28, 258-263.

Palanza P, Gioiosa L, vom Saal FS, Parmigiani S. (2008). Effects of developmental exposure to bisphenol A on brain and behavior in mice. Environ Res. 108, 150-157.

Partosch F, Mielke H, Gundert-Remy U (2013) Functional UGT-glucuronyltransferase 2B15 polymorphism and Bisphenol A concentrations in blood: results from physiologically based kinetic modelling Arch Toxicol 87, 1257-1264.

Patisaul H. (2010) Assessing risks from bisphenol-A. Evaluating human health risks from endocrine disruptors such as BPA is difficult, but animal studies suggest trouble is afoot. American Scientist 98, 30.

Patisaul HB, Bateman HL. (2008). Neonatal exposure to endocrine active compounds or an ERbeta agonist increases adult anxiety and aggression in gonadally intact male rats. Horm Behav. 53, 580-588.

Patisaul HB, Sullivan AW, Radford ME, Walker DM, Adewale HB, Winnik B, Coughlin JL, Buckley B, Gore AC. (2012) Anxiogenic effects of developmental bisphenol A exposure are associated with gene expression changes in the juvenile rat amygdala and mitigated by soy.PLoS One. 7, e43890.

Patisaul HB, Todd KL, Mickens JA, Adewale HB. (2009). Impact of neonatal exposure to the ERalpha agonist PPT, bisphenol-A or phytoestrogens on hypothalamic kisspeptin fiber density in male and female rats. Neurotoxicology. 30, 350-357.

Patterson TA, Twaddle NC, Roegge CS, Callicott RJ, Fisher JW and Doerge DR, (2013). Concurrent determination of bisphenol A pharmacokinetics in maternal and fetal rhesus monkeys. Toxicology and Applied Pharmacology, 267, 41-48.

Philippat C, Mortamais M, Chevrier C, Petit C, Calafat AM, Ye X, Silva MJ, Brambilla C, Pin I, Charles MA, Cordier S, Slama R. (2012) Exposure to phthalates and phenols during pregnancy and offspring size at birth. Environ Health Perspect. 120, 464-470. Erratum in: Environ Health Perspect. 120, 470, 2012.

Plastics Europe - Polycarbonate/BPA Group. (2007). Applications of Bisphenol-A. www.bisphenol-a-europe.org/uploads/BPA\%20applications.pdf

Pottenger LH, Domoradzki JY, Markham DA, Hansen SC, Cagen SZ, Waechter JM Jr. (2000). The relative bioavailability and metabolism of bisphenol $A$ in rats is dependent upon the route of administration. Toxicol Sci 54, 3-18.

Prins GS, Ye SH, Birch L, Ho SM, Kannan K. (2011) Serum bisphenol A pharmacokinetics and prostate neoplastic responses following oral and subcutaneous exposures in neonatal Sprague-Dawley rats. Reprod Toxicol. 31, 1-9.

Ravoori S, Feng Y, Neale JR, Jeyabalan J, Srinivasan C, Hein DW, Gupta RC (2008). Dose-dependent reduction of 3,2'-dimethyl-4-aminobiphenyl-derived DNA adducts in colon and liver of rats administered celecoxib Mutat Res. 638:103-9.

Rhomberg LR, Goodman JE. (2012) Low dose effects and nonmonotonic responses of endocrine disrupting chemicals: has the case been made? Regul Toxicol Pharmacol. 64, 130-133, 2012. 
Riu A, Grimaldi M, Le Maire A, Bey G, Phillips K, Boulahtouf A, Perdu E, Zalko D, Bourguet W, Balaguer P. (2011) Peroxisome proliferator-activated receptor $Y$ is a target for halogenated analogs of bisphenol A. Environ. Health Perspect. 119, 1227-1232.

Rochester JR. (2013) Bisphenol A and human health: A review of the literature. Reprod. Toxicol. 42, 132-155.

Rönn M, Kullberg J, Karlsson H, Berglund J, Malmberg F, Orberg J, Lind L, Ahlstrom H and Lind PM. (2013) Bisphenol A exposure increases liver fat in juvenile fructose-fed Fischer 344 rats. Toxicology, 303, 125-132.

Ropero A, Alonso Magdalena P, García-García E, Ripoll C, Fuentes E, Nadal A. (2008) Bisphenol A disruption of the endocrine pancreas and blood glucose homeostasis. Int. J. Androl. 31, 194-200.

Roy A, Bauer SM, Lawrence BP. (2012) Developmental exposure to bisphenol A modulates innate but not adaptive immune responses to influenza $A$ virus infection. PLoS ONE 7, e38448.

Rubin B S, Murray M K, Damassa D A, King J C, Soto A M (2001) Perinatal exposure to low doses of bisphenol A affects body weight, patterns of estrous cyclicity, and plasma LH levels. Environ Health Perspect, 109, 675-680.

Rudel RA, Camann DE, Spengler JD, Korn LR and Brody JG (2003) Phthalates, alkylphenols, pesticides, polybrominated diphenyl ethers, and other endocrine-disrupting compounds in indoor air and dust. Environ Sci Technol 37, 4543-4555.

Rwei SP, Kao SC, Liou GS, Cheng KC, Guo W. (2003) Curing and pyrolysis of epoxy resins containing 2-(6-oxido-6H-dibenz(c, e) $(1,2)$ oxaphosphorin-6-yl)-1,4naphthalenediol or bisphenol S. Colloid Polym. Sci. 281, 407-415.

Ryan K K, Haller A M, Sorrell J E, Woods S C, Jandacek R J, Seeley R J (2010) Perinatal exposure to bisphenol-A and the development of metabolic syndrome in $C D-1$ mice. Endocrinology 151, 2603-2612.

Ryan BC, Vandenbergh JG. (2006). Developmental exposure to environmental estrogens alters anxiety and spatial memory in female mice. Horm Behav. 50, 85-93.

Sajiki J, Hasegawa $Y$, Hashimoto $H$, Makabe $Y$, Miyamoto F, Yanagibori R, Shin J, Shimidzu $Y$ and Morigami T (2008) Determination of bisphenol A (BPA) in plasma of hemodialysis patients using three methods: LC/ECD, LC/MS, and ELISA. Toxicology Mechanisms and Methods 18: 733-738.

Sajiki J, Yonekubo J (2004). Leaching of bisphenol A (BPA) from polycarbonate plastic to water containing amino acids and its degradation by radical oxygen species. Chemosphere, 55, 861-867.

Sakurai H, Maeda M, Miyahara K, Nakayama M (2002). Extraction of bisphenol-A from cardiopulmonary bypass circuit. Kyobu Geka 55(9):770-772 (only abstract available in english)

Sargis R M, Johnson D N, Choudhury R A, Brady M J (2010) Environmental endocrine disruptors promote adipogenesis in the 3T3-L1 cell line through glucocorticoid receptor activation. Obesity (Silver Spring, Md.) 18, 1283-1288.

Sasaki N, Okuda K, Kato T, Kakishima H, Okuma H, Abe H, Tuchida K, Kubono K (2005) Salivary bisphenol-A levels detected by ELISA after restoration with composite resin. J Mater Sci: Mater Med 16, 297 - 300.

Satoh K, Ohyama K, Aoki N, Iida M, Nagai F. (2004). Study on anti-androgenic effects of bisphenol a diglycidyl ether (BADGE), bisphenol $F$ diglycidyl ether (BFDGE) and their derivatives using cells stably transfected with human androgen receptor, AR-EcoScreen. Food Chem Toxicol.42, 983-993. 
Savage JH, Matsui EC, Wood RA, Keet CA (2012) Urinary levels of triclosan and parabens are associated with aeroallergen and food sensitization. J Allergy Clin Immunol, 130, 453-460 e457

SCENIHR (2008). Opinion on the safety of medical devices containing DEHP plasticized PVC or other plasticizers on neonates and other groups possibly at risk. http://ec.europa.eu/health/archive/ph_risk/committees/04_scenihr/docs/scenihr_o_014. pdf

SCENIHR (2012) Memorandum on the use of the scientific literature for human health risk assessment purposes - weighing of evidence and expression of uncertainty.http://ec.europa.eu/health/scientific_committees/emerging/docs/scenihr_s_0 01.pdf

Schönfelder G, Wittfoht W, Hopp H, Talsness CE, Paul M, Chahoud I. (2002). Parent bisphenol $A$ accumulation in the human maternal fetal-placental unit. Environ Health Perspect 110, A703-A707.

Schöringhumer K, Cichna-MarkI M. (2007). Sample clean-up with sol-gel enzyme and immunoaffinity columns for the determination of bisphenol $A$ in human urine. J Chromatogr B Analyt Technol Biomed Life Sci. 850, 361-369.

Schmalz G, Preiss A, Arenholtt-Bindslev D (1999) Bisphenol A content of resin monomers and related degradation products. Clin Oral Investig 3, 114-119.

Seki N, Nakajima M, Kishikawa R, Hosaka K, Foxton RM, Tagami J (2011). The influence of light intensities irradiated directly and indirectly through resin composite to self-etch adhesives on dentin bonding. Dent Mater J. 30, 315-322.

Shankar A and Teppala S. (2012a) Urinary bisphenol A and hypertension in a multiethnic sample of US adults. J Environ Public Health, 2012, 481641.

Shankar A, Teppala S and Sabanayagam C. (2012b) Bisphenol A and Peripheral Arterial Disease: Results from the NHANES. Environmental Health Perspectives, 120, 1297-1300.

Shankar A, Teppala S and Sabanayagam C. (2012c). Urinary bisphenol a levels and measures of obesity: results from the national health and nutrition examination survey 2003-2008. ISRN Endocrinol, 2012, 965243.

Shelnutt S, Kind J, Allaben W (2013). Bisphenol A: Update on newly developed data and how they address NTP's 2008 finding of "some concern". Food Chem Toxicol 57, 284295.

Sheng ZG, Tang Y, Liu YX, Yuan Y, Zhao BQ, Chao XJ and Zhu BZ. (2012). Low concentrations of bisphenol a suppress thyroid hormone receptor transcription through a nongenomic mechanism. Toxicology and Applied Pharmacology, 259, 133-142.

Shimizu M, Ohta K, Matsumoto Y, Fukuoka M, Ohno Y and Ozawa S, (2002). Sulphation of bisphenol $A$ abolished its estrogenicity based on proliferation and gene expression in human breast cancer MCF-7 cells. Toxicol In vitro 16, 549-556.

Shin BS, Kim CH, Jun YS, Kim DH, Lee BM, Yoon CH, Park EH, Lee KC, Han SY, Park KL, Kim HS, Yoo SD. (2004). Physiologically based pharmacokinetics of bisphenol A. J. Toxicol. Environ. Health A 67:1971-1985.

Shin BS, Hwang SW, Bulitta JB, Lee JB, Yang SD, Park JS, Kwon MC, Kim do J, Yoon HS and Yoo SD. (2010) Assessment of bisphenol A exposure in Korean pregnant women by physiologically based pharmacokinetic modeling. J Toxicol Environ Health A, 73, 15861598.

Shintani H and Hayashi F (2011) Determination of the Endocrine Disrupter Bisphenoi-A in the Blood of Uremia Patients Treated by Dialysis. Pharm Anal Acta S11:001. doi:10.4172/2153-2435.S11-001 
Shintani H. (2001). Determination of the endocrine disrupter Bisphenol-A in the bllod of uremia patients treated by dialysis. Chromatographia 53 (5/6(331-333).

Shintani H, Suzuki E, Sakurai M (2003). Determination of compounds inhibiting bacterial growth ins sterilized medical devices. Chromatographia 58 (3/4): 193-199.

Sieli P T, Jašarevic E, Warzak DA, Mao J, Ellersieck MR, Liao C, Kannan K, Collet SH, Toutain PL, vom Saal FS, Rosenfeld CS. (2011) Comparison of serum bisphenol A concentrations in mice exposed to bisphenol $A$ through the diet versus oral bolus exposure Envir Health Perspect 119, 1260-1265.

Silver MK, O'Neill MS, Sowers MR and Park SK. (2011) Urinary bisphenol A and type-2 diabetes in U.S. adults: data from NHANES 2003-2008. PLoS One, 6, e26868.

Snyder RW, Maness SC, Gaido KW, Welsch F, Sumner SC], Fennell TR. (2000). Metabolism and disposition of bisphenol $A$ in female rats. Toxicol Appl Pharmacol 168:225-234.

Socialstyrelsen (2012) (Swedish National Board of Health and Welfare), Bisfenol A i dentala material. - Bisphenol A in dental materials 2012-6-48. ISBN:978-91-87169-489, 59pp. http://www.socialstyrelsen.se/publikationer2012/2012-6-48

Somm E, Schwitzgebel V M, Toulotte A, Cederroth C R, Combescure C, Nef S, Aubert M L, Hüppi P S (2009) Perinatal exposure to bisphenol A alters early adipogenesis in the rat. Environmental Health Perspectives 117,1549-1555, 2009.

Song L, Xia W, Zhou Z, Li Y, Lin Y, Wei J, Wei Z, Xu B, Shen J, Li W and Xu S. (2012) Low-level phenolic estrogen pollutants impair islet morphology and beta-cell function in isolated rat islets. J Endocrinol, 215, 303-311.

Soriano S, Alonso-Magdalena P, García-Arévalo M, Novials A, Muhammed SJ, Salehi A, Gustafsson JA, Quesada I, Nadal A. (2012) Rapid insulinotropic action of low doses of bisphenol-A on mouse and human islets of Langerhans: role of estrogen receptor $\beta$. PLoS One. 7, e31109.

Spanier AJ, Kahn RS, Kunselman AR, Hornung R, Xu Y, Calafat AM, Lanphear BP (2012) Prenatal exposure to bisphenol $A$ and child wheeze from birth to 3 years of age. Environmental Health Perspectives, 120, 916-920.

Spitsbergen JC, Loewrigkeit P, Bluestein C, Sugarman J, Lauze WL. (1971) 26th Annual Techn. Conf. Reinforced Plastics/Composites Division, The Society of the Plastics Industry, Inc., Section 19-C, 1

Stachel B, Ehrhorn U, Heemken OP, Lepom P, Reincke H, Sawal O, Theobald N. (2003) Xenoestrogens in the River Elbe and its tributaries. Environ. Pollut. 124, 497-507.

Stahlhut RW, Welshons WV, Swan SH. (2009) Bisphenol A data in NHANES suggest longer than expected half-life, substantial non-food exposure, or both. Environ Health Perspect. 2009;117:784-789.

Staples CA, Dorn PB, Klecka GM, Oblock ST and Harris LR (1998) A review of the environmental fate, effects, and exposures of bisphenolA. Chemosphere 36: 2149-2173.

Stump DG, Beck MJ, Radovsky A, Garman RH, Freshwater L, Sheets LP, Marty MS, Waechter JM, Dimond SS, Van Miller JP, Shiotsuka RN, Beyer D, Chappelle AH, Hentges SG, (2010). Developmental neurotoxicity study of dietary bisphenol A in Sprague-Dawley rats. Toxicological Sciences 115, 167-182.

Sugiura-Ogasawara M, Ozaki Y, Sonta S, Makino T, Suzumori K. (2005). Exposure to bisphenol $A$ is associated with recurrent miscarriage. Human Reproduction (Oxford, England), 20, 2325-2329.

Sun Y, Wada M, Kuroda, N, Hirayama K, Nakazawa H and Nakashima K (2001) Simultaneous determination of phenolic xenoestrogens by solid-phase extraction and 
high-performance liquid chromatography with fluorescence detection. Analytical Sciences 17: 697-702.

Sun Y, Irie M, Kishikawa N, Wada M, Kuroda N, Nakashima K (2004). "Determination of bisphenol $A$ in human breast milk by HPLC with column-switching and fluorescence detection." Biomed Chromatogr 18(8): 501-507.

Sunitha C, Kailasam V, Padmanabhan S, Chitharanjan AB (2011). Bisphenol A release from an orthodontic adhesive and its correlation with the degree of conversion on varying light-curing tip distances. Amer J Orthodont Dentofac Orthoped, 140, 239-244.

Susiarjo M, Hassold TJ, Freeman E, Hunt PA.(2007). Bisphenol A exposure in utero disrupts early oogenesis in the mouse. PLoS Genet. 3 (1):e5.

Suzuki K, Ishikawa K, Sugiyama K, Furuta H, Nishimura F. (2000). Content and release of bisphenol A from polycarbonate dental products. Dent Mater J 19, 389-395.

Takahashi A, Higashino F, Aoyagi M, Kyo S, Nakata T, Noda M, Shindo M, Kohgo T, Sano $H$ (2004). Bisphenol A from dental polycarbonate crown upregulates the expression of hTERT. J Biomed Mat Res, 71B, 214-221.

Takashima Y et al. (2001). Lack of effects of bisphenol A in maternal rats or treatment on response of their offspring to $\mathrm{N}$-nitrosobis(2-hydroxypropyl)amine. Journal of Toxicologic Pathology, 14:87-98).

Takeuchi T, Tsutsumi O, Ikezuki Y, Takai Y, Taketani Y. (2004). Positive relationship between androgen and the endocrine disruptor, bisphenol $A$, in normal women and women with ovarian dysfunction. Endocrine Journal, 51(2):165-169.

Tanabe N, Yoshino H, Kimoto T, Hojo Y, Ogiue-Ikeda M, Shimohigashi $\mathrm{Y}$ and Kawato S. (2012). Nanomolar dose of bisphenol A rapidly modulates spinogenesis in adult hippocampal neurons. Molecular and Cellular Endocrinology, 351, 317-325.

Tang WY, Morey LM, Cheung YY, Birch L, Prins GS, Ho SM (2012) Neonatal exposure to estradiol/bisphenol A alters promoter methylation and expression of Nsbp1 and Hpcal1 genes and transcriptional programs of Dnmt3a/b and $\mathrm{Mbd} 2 / 4$ in the rat prostate gland throughout life. Endocrinology. 153, 42-55.

Tayama S, Nakagawa Y, Tayama K. (2008) Genotoxic effects of environmental estrogenlike compounds in CHO-K1 cells. Mutat Res. 649, 114-125.

Taylor J A, Welshons WV, Vom Saal FS (2008) No effect of route of exposure (oral; subcutaneous injection) on plasma bisphenol A throughout $24 \mathrm{~h}$ after administration in neonatal female mice Reproductive Toxicology 25, 169-176.

Taylor J A, Vom Saal F S, Welshons W V, Drury B, Rottinghaus G, Hunt P A, Toutain P L, Laffont C M, VandeVoort C A, (2011). Similarity of bisphenol A pharmacokinetics in rhesus monkeys and mice: relevance for human exposure. Environ. Health Persp. 119, 422-430.

Teeguarden JG, Waechter JM Jr, Clewell HJ 3rd, Covington TR, Barton HA. (2005). Evaluation of oral and intravenous route pharmacokinetics, plasma protein binding, and uterine tissue dose metrics of bisphenol A: A physiologically based pharmacokinetic approach. Toxicol Sci 85, 823-838.

Teeguarden J, Hanson-Drury S, Fisher JW, Doerge DR (2013) Are typical human serum BPA concentrations measureable and sufficient to be estrogenic in the general population? Food Chem Toxicol. 62, 949-963.

Teeguarden J G, Calafat A M, Ye X, Doerge D R, Churchwell M I, Gunawan R, Graham M $K$ (2011) Twenty-four hour human urine and serum profiles of bisphenol a during highdietary exposure. Toxicol Sci. 123, 48-57 (2011). 
Terasaki M, Nomachi M, Edmonds JS, Morita M. (2004) Impurities in industrial grade 4,4'-isopropylidene diphenol (bisphenol A): possible implications for estrogenic activity. Chemosphere. 2004 May; 55(6):927-31. PubMed PMID: 15041297.

Terasaki M, Shiraishi F, Nishikawa T, Edmonds JS, Morita M, Makino M. Estrogenic activity of impurities in industrial grade bisphenol A. Environ SciTechnol. 2005 May $15 ; 39(10): 3703-7$

Tharp AP, Maffini MV, Hunt PA, VandeVoort CA, Sonnenschein C and Soto AM. (2012) Bisphenol A alters the development of the rhesus monkey mammary gland. Proceedings of the National Academy of Sciences of the United States of America, 109, 8190-8195.

Tian YH, Baek JH, Lee SY, Jang CG. (2010) Prenatal and postnatal exposure to bisphenol a induces anxiolytic behaviors and cognitive deficits in mice. Synapse. 64, 432-439.

Timms BG, Howdeshell KL, Barton L, Bradley S, Richter CA, vom Saal FS. (2005) Estrogenic chemicals in plastic and oral contraceptives disrupt development of the fetal mouse prostate and urethra. Proc Natl Acad Sci U S A. 102, 7014-7019.

Tiwari D, Kamble J, Chilgunde S, Patil P, Maru G, Kawle D, Bhartiya U, Joseph L, Vanage G. (2012) Clastogenic and mutagenic effects of bisphenol A: an endocrine disruptor. Mutation Research, 743, 83-90.

Tiwari D and Vanage G. (2013). Mutagenic effect of Bisphenol A on adult rat male germ cells and their fertility. Reproductive Toxicology, 40, 60-68.

Tominaga T, Negishi T, Hirooka H, Miyachi A, Inoue A, Hayasaka I, Yoshikawa Y, (2006). Toxicokinetics of bisphenol $A$ in rats, monkeys and chimpanzees by the LC-MS/MS method Toxicology 226, 208-217.

Trasande, L., Attina TM, Blustein J. (2012) Association between urinary bisphenol A concentration and obesity prevalence in children and adolescents. JAMA, 308, 11131121.

Trdan Luŝin T, Roskar R and Mrhar A. (2012) Evaluation of bisphenol A glucuronidation according to UGT1A1*28 polymorphism by a new LC-MS/MS assay. Toxicology, 292, 3341.

Tsukioka T, Terasawa J, Sato S, Hatayama Y, Makino T, Nakazawa H. (2004). Development of analytical method for determining trace amounts of BPA in urine samples and estimation of exposure to BPA. J Environ Chem 14:57-63.

Tsutsui T, Tamura $Y$, Suzuki A, Hirose $Y$, Kobayashi M, Nishimura $H$, Metzler M, Barrett JC. (2000) Mammalian cell transformation and aneuploidy induced by five bisphenols. Int J Cancer. 86, 151-154.

Tsutsui T, Tamura Y, Yagi E, Hasegawa K, Takahashi M, Maizumi N, Yamaguchi F, Barrett JC. (1998) Bisphenol-A induces cellular transformation, aneuploidy and DNA adduct formation in cultured Syrian hamster embryo cells. Int J Cancer.75, 290-294.

Twaddle NC, Churchwell MI, Vanlandingham M, Doerge DR. (2010). Quantification of deuterated bisphenol $A$ in serum, tissues, and excreta from adult Sprague-Dawley rats using liquid chromatography with tandem mass spectrometry. Rapid Commun Mass Spectrom 24, 3011-3020.

Tyl RW, Myers CB, Marr MC, Sloan CS, Castillo NP, Veselica MM, Seely JC, Dimond SS, Van Miller JP, Shiotsuka RN, Beyer D, Hentges SG, Waechter JM Jr. (2008) Twogeneration reproductive toxicity study of dietary bisphenol A in CD-1 (Swiss) mice. Toxicol Sci. 104, 362-384.

Tyl RW, Myers CB, Marr MC, Castillo NP, Veselica MM, Joiner RL, Dimond SS, Van Miller JP, Stropp GD, Waechter JM, Hentges SG. (2008) One-generation reproductive toxicity study of dietary 17beta-estradiol (E2; CAS No. 50-28-2) in CD-1 (Swiss) mice. Reprod Toxicol 25, $144-160$. 
Tyl RW, Myers CB, Marr MC, Thomas BF, Keimowitz AR, Brine DR, Veselica MM, Fail PA, Chang TY, Seely JC, Joiner RL, Butala JH, Dimond SS, Cagen SZ, Shiotsuka RN, Stropp GD, Waechter JM. (2002) Three-generation reproductive toxicity study of dietary bisphenol A in CD Sprague-Dawley rats. Toxicol Sci. 68, 121-146.

Ulutaş OK, Yildiz N, Durmaz E, Ahbab MA, Barlas N and Cok I. (2011). An in vivo assessment of the genotoxic potential of bisphenol $A$ and 4-tert-octylphenol in rats. Archives of Toxicology, 85, 995-1001.

Upmeier A, Degen GH, Diel P, Michna H, Bolt HM. (2000). Toxicokinetics of bisphenol A in female DA/Han rats after single i.v. and oral administration. Arch Toxicol 74:431-436.

US FDA (Food and Drug Administration), 2010a. Update on Bisphenol A for Use in Food Contact Applications: January 2010 Available from http://www.fda.gov/NewsEvents/PublicHealthFocus/ucm197739.htm

US FDA (Food and Drug Administration), (2010b). Memorandum of 11/16/2009, Summary of Bisphenol A Biommonitoring Studies [FDA-2010-N-0100-0001] Available from http://www.regulations.gov/search/Regs/home.htmL\# docketDetail?R=FDA-2010-N0100

US FDA (US Food and Drug Administration) Safety, (2002). Assessment of di(2ethylhexyl)phthalate (DEHP) released from medical devices. Center for Devices and Radiological Health, 2002.

US FDA, (2013). Bisphenol A (BPA): Use in food contact application. US Food and Drug Administration, Silver Spring, MD, USA. <http://www.fda.gov/NewsEvents/

US NTP (1982). Carcinogenesis bioassay of bisphenol A (CAS No. 80-05-7) in F344 rats and B6C3F1 mice (feed study). Research Triangle Park, NC, United States Department of Health and HumanServices, National Toxicology Program (TR-215; http://ntp.niehs.nih.gov/go/14366).

USA National Toxicology Program (NTP, 2008) NTP-CERHR (2008). Monograph on the Potential Human Reproductive and Developmental Effects of Bisphenol A. http://ntp.niehs.nih.gov/ntp/ohat/bisphenol/bisphenol.pdf

Vallo CI, Schroeder WF (2005). Properties of acrylic bone cements formulated with BisGMA. J Biomed Mat Res Part B: Appl Biomat. 748, 676-685.

Vandenberg LN, Hauser R, Marcus M, Olea N, Welshons WV (2007). Human exposure to bisphenol A (BPA). Reprod Toxicol 24: 139-177.

Vandenberg LN, Maffini MV, Schaeberle CM, Ucci AA, Sonnenschein C, Rubin BS, Soto AM.(2008) Perinatal exposure to the xenoestrogen bisphenol-A induces mammary intraductal hyperplasias in adult CD-1 mice. Reprod Toxicol. 26, 210-219.

Vandenberg LN, Chauhoud I, Heindel JJ, Padmanabhan V, Paumgartten FJ, Schoenfelder G. (2010a). Urinary, circulating and tissue biomonitoring studies indicate widespread exposure to bisphenol A. Environ Health Perspect 118, 1055-1070.

Vandenberg LN, Chahoud I, Padmanabhan V, Paumgartten FJ, Schoenfelder G. (2010b). Biomonitoring studies should be used by regulatory agencies to assess human exposure levels and safety of bisphenol A. Environ Health Perspect 118, 1051-1054.

Vandenberg LN, Colborn T, Hayes TB, Heindel JJ, Jacobs DR Jr, Lee DH, Shioda T, Soto AM, Von Saal FS, Welshons WV, Zoeller RT, Myers JP. (2012) Hormones and endocrinedisrupting chemicals: low-dose effects and nonmonotonic dose responses. Endocr Rev $33,378-455$.

Vandenberg LN, Gerona RR, Kannan K, Taylor JA, van Breemen RB, Dickenson CA, Liao C, Yuan Y, Newbold RR, Padmanabhan V, Vom Saal FS, Woodruff TJ. (2014)A round robin approach to the analysis of bisphenol A (BPA) in human blood samples. Environ Health. 13, 25 
Vandenberg LN, Schaeberle CM, Rubin BS, Sonnenschein C, Soto AM. (2013) The male mammary gland: a target for the xenoestrogen bisphenol A. Reprod Toxicol. 37, 15-23.

Vandentorren S, Morin L, Sarter H, Bidondo ML, Oleko A and Leridon H (2011) Bisphenol$A$ and phthalates contamination of urine samples by catheters in the Elfe pilot study: Implications for large-scale biomonitoring studies. Environmental research. 06:761-64.

Van Landuyt KL, Nawrot T, Geebelen B, De Munck J, Snauwaert J, Yoshihara K, Scheers H, Godderis L, Hoet P, Van Meerbeek B (2011) How much do resin-based dental materials release? A meta-analytical approach. Dental Materials, 27, 723-747.

Van Landuyt KL, Nawrot T, Geebelen B, De Munck J, Snauwaert J, Yoshihara K, Scheers H, Godderis L, Hoet P, Van Meerbeek B. (2013) Corrigendum to "How much do resinbased dentalmaterials release? A meta-analytical approach" [Dental 27 (8) (2011) 723747] Dental materials 29, 919.

Viberg H, Fredriksson A, Buratovic S and Eriksson P. (2011) Dose-dependent behavioral disturbances after a single neonatal Bisphenol A dose. Toxicology, 290, 187-194.

Viñas P, Campillo N, Martínez-Castillo N, Hernández-Córdoba M. (2010) Comparison of two derivatization-based methods for solid-phase microextraction-gas chromatographymass spectrometric determination of bisphenol $\mathrm{A}$, bisphenol $\mathrm{S}$ and biphenol migrated from food cans. Anal Bioanal Chem. 397, 115-125

Vinggaard AM, Korner W, Lund $\mathrm{KH}$, Bolz $\mathrm{U}$ and Petersen $\mathrm{JH}$ (2000) Identification and quantification of estrogenic compounds in recycled and virgin paper for household use as determined by an in vitro yeast estrogen screen and chemical analysis. Chem Res Toxicol 13: $1214-1222$.

Völkel W, Bittner N and Dekant W, (2005). Quantitation of Bisphenol A and Bisphenol A Glucuronide in Biological Samples by High Performance Liquid Chromatography-Tandem Mass Spectrometry. Drug metabolism and disposition: the biological fate of chemicals 33, 1748-1757, 2005.

Völkel W, Colnot T, Csanady GA, Filser JG and Dekant W, (2002). Metabolism and kinetics of bisphenol $a$ in humans at low doses following oral administration. Chem.Res.Toxicol. 15, 1281-1287,2002.

Völkel W, Kiranoglu M, and Fromme H (2008) Determination of free and total bisphenol A in human urine to assess daily uptake as a basis for a valid risk assessment. Toxicol Lett 179: 155-162, 2008.

Von Goetz N, Wormuth M, Scheringer M and Hungerbühler K (2010) Bisphenol A: How the most relevant exposure sources contribute to total consumer exposure. Risk Anal 30: 473-487.

Wada K., Sakamoto H., Nishikawa K., Sakuma S., Nakajima A., Fujimoto Y., Kamisaki Y.: Life style-related diseases of the digestive system: endocrine disruptors stimulate lipid accumulation in target cells related to metabolic syndrome. Journal of Pharmacological Sciences 105: 133-137 (2007).

Wang T, Li M, Chen B, Xu M, Xu Y, Huang Y, Lu J, Chen Y, Wang W, Li X, Liu Y, Bi Y, Lai S, Ning G. (2012) Urinary bisphenol A (BPA) concentration associates with obesity and insulin resistance. J Clin Endocrinol Metab. 97, E223-7. doi: 10.1210/jc.2011-1989. Epub 2011 Nov 16.

Wang J, Sun B, Hou M, Pan X, Li X. (2013) The environmental obesogen bisphenol A promotes adipogenesis by increasing the amount of $11 \beta$-hydroxysteroid dehydrogenase type 1 in the adipose tissue of children. Int J Obes (Lond). 37, 999-1005.

Watanabe M, Hase T, Imai Y. (2001) Change in the bisphenol A content in a polycarbonate orthodontic bracket and its leaching characteristics in water. Dent Mater J.; 20, 353-358. 
Watanabe M, Fukazawa H, Shiraishi F, Shiraishi H, Shiozawa T, Terao T. (2004) Analysis and estrogenic activity of bisphenol $A$ and other chemicals released from waste paper by pulping. J. Environ. Chem. 14, 65-71.

Watanabe M. (2004) Degradation and formation of bisphenol A in polycarbonate used in dentistry. J Med Dent Sci. 51:1-6.

Weber Lozada K, Keri RA. (2011) Bisphenol A increases mammary cancer risk in mouse models of breast cancer. Biol Reprod. 85, 490-497.

Wei J., Lin Y., Li Y., Ying Ch., Chen J., Song L., Zhou Z., Lv Z., Xia W., Chen X., Xu S. (2011) Perinatal exposure to bisphenol $A$ at reference dose predisposes offspring to metabolic syndrome in adult rats on a high fat diet. Endocrinology 152, 3049-3061 (2011)

WHO (2010) Joint FAO/WHO Expert Meeting to Review Toxicological and Health Aspects of Bisphenol A. Summary report. Available from:

http://www.who.int/foodsafety/chem/chemicals/bisphenol_release/en/index.htmL

WHO/FAO (2010) Joint FAO/WHO Expert Meeting to Review Toxicological and Health Aspects of Bisphenol A. Summary Report including Report of Stakeholder Meeting on Bisphenol A. http://www.who.int/foodsafety/chem/chemicals/BPA_Summary2010.pdf

Willhite CC, Ball GL, McLellan CJ. (2008). Derivation of a bisphenol A oral reference dose (RfD) and drinking-water equivalent concentration. J Toxicol Environ Health B Crit Rev $11,69-146$.

Wilson NK, Chuang JC, Morgan MK, Lordo RA, and Sheldon, LS (2007) An observational study of potential exposures of preschool children to pentachlorophenol, Bisphenol-A, and nonylphenol at home and daycare. Environ Res 103: 9-20.

Wolff MS, Engel SM, Berkowitz GS, Ye X, Silva MJ, Zhu C, Wetmur J, Calafat AM. (2008b). Prenatal phenol and phthalate exposures and birth outcomes. Environmental Health Perspectives, 116, 1092-1097.

Wolff MS, Teitelbaum SL, Windham G, Pinney SM, Britton JA, Chelimo C, Godbold J, Biro F, Kushi LH, Pfeiffer CM, Calafat AM. (2007). Pilot study of urinary biomarkers of phytoestrogens, phthalates, and phenols in girls. Environmental Health Perspectives, $115,116-121$.

Wolff MS, Britton JA, Boguski L, Hochman S, Maloney N, Serra N, Liu Z, Berkowitz G, Larson S, Forman J. (2008a). Environmental exposures and puberty in inner-city girls. Environmental Research, 107, 393-400.

Wolff MS, Teitelbaum SL, Pinney SM, Windham G, Liao L, Biro F, Kushi LH, Erdmann C, Hiatt RA, Rybak ME, Calafat AM; Breast Cancer and Environment Research Centers. (2010). Investigation of relationships between urinary biomarkers of phytoestrogens, phthalates, and phenols and pubertal stages in girls. Environmental Health Perspectives, $118,1039-1046$.

Wolstenholme, JT, Rissman EF and Connelly JJ. (2011) The role of Bisphenol A in shaping the brain, epigenome and behavior. Hormones and Behavior,59, 296-305.

Wolstenholme JT, Edwards M, Shetty SR, Gatewood JD, Taylor JA, Rissman EF and Connelly JJ. (2012) Gestational exposure to bisphenol a produces transgenerational changes in behaviors and gene expression. Endocrinology, 153, 3828-3838.

Xu X, Ye Y, Li T, Chen L, Tian D, Luo Q and Lu M. (2010) Bisphenol-A rapidly promotes dynamic changes in hippocampal dendritic morphology through estrogen receptormediated pathway by concomitant phosphorylation of NMDA receptor subunit NR2B. Toxicology and Applied Pharmacology, 249, 188-196. 
Xu XH, Wang YM, Zhang J, Luo QQ, Ye YP and Ruan Q, (201)0. Perinatal exposure to bisphenol-A changes $\mathrm{N}$-methyl-D-aspartate receptor expression in the hippocampus of male rat offspring. Environmental toxicology and chemistry, 29, 176-181.

Xu X, Tian D, Hong X, Chen L and Xie L. (2011) Sex-specific influence of exposure to bisphenol-A between adolescence and young adulthood on mouse behaviors. Neuropharmacology, 61, 565-573.

Xu X, Hong X, Xie L, Li T, Yang Y, Zhang Q, Zhang G and Liu X. (2012) Gestational and lactational exposure to bisphenol-A affects anxiety- and depression-like behaviors in mice. Hormones and Behavior, 62, 480-490.

Xu X, Xie L, Hong X, Ruan Q, Lu H, Zhang Q, Zhang G and Liu X. (2013). Perinatal exposure to bisphenol-A inhibits synaptogenesis and affects the synaptic morphological development in offspring male mice. Chemosphere 91, 1073-1081.

Xu X, Liu X, Zhang Q, Zhang G, Lu Y, Ruan Q, Dong F, Yang Y. (2013) Sex-specific effects of bisphenol-A on memory and synaptic structural modification in hippocampus of adult mice.) Horm Behav. 2013 May;63(5):766-75. doi: 10.1016/j.yhbeh.2013.03.004. Epub 2013 Mar 19.

Yamasaki K, Takeyoshi Y, Yakabe Y, Sawaki M, Imatanaka N, Takatsuki M. (2002) Comparison of gene reporter assay and immature rat uterotrophic assay of twenty-three chemicals. Toxicology 170, 21-30.

Yamasaki H, Nagake Y, Makino H (2001). Determination of Bisphenol A in Effluents of Hemodialyzers. Nephron 88, 376-378.

Yang M, Ryu JH, Jeon R, Kang D, Yoo KY. (2009). Effects of bisphenol A on breast cancer and its risk factors. Archives of Toxicology 83, 281-285.

Yang X, Doerge DR and Fisher JW, (2013) Prediction and evaluation of route dependent dosimetry of BPA in rats at different life stages using a physiologically based pharmacokinetic model. Toxicology and Applied Pharmacology, 270, 45-59.

Ye X, Bishop AM, Needham LL, Calafat AM. (2008). "Automated on-line column-switching HPLC-MS/MS method with peak focusing for measuring parabens, triclosan, and other environmental phenols in human milk." Anal Chim Acta 622, 150-156.

Ye X, Bishop AM, Reidy JA, Needham LL, Calafat AM. (2007). Temporal stability of the conjugated species of bisphenol $A$, parabens, and other environmental phenols in human urine. J Expo Sci Environ Epidemiol 17:567-572.

Ye X, Kuklenyik Z, Needham LL, Calafat AM. (2005). Quantification of urinary conjugates of bisphenol A, 2,5-dichlorophenol, and 2-hydroxy-4-methoxybenzophenone in humans by online solid phase extraction-high performance liquid chromatographytandem mass spectrometry. Anal Bioanal Chem 383:638-644.

Ye X, Kuklenyik Z, Needham L L, Calafat A M. (2006). Measuring environmental phenols and chlorinated organic chemicals in breast milk using automated on-line columnswitching-high performance liquid chromatography-isotope dilution tandem mass spectrometry. J Chromatogr B Analyt Technol Biomed Life Sci 831, 110-115.

Ye X, Wong LY, Bishop AM and Calafat AM. (2011) Variability of urinary concentrations of bisphenol A in spot samples, first morning voids, and 24-hour collections. Environmental Health Perspectives, 119, 983-988.

Yi B, Kim C, Yang M (2010). "Biological monitoring of bisphenol A with HLPC/FLD and LC/MS/MS assays." J Chromatogr B Analyt Technol Biomed Life Sci 878, 2606-2610.

Yoshida M, Shimomoto T, Katashima S, Watanabe G, Taya K, Maekawa A. (2004). Maternal exposure to low doses of bisphenol a has no effects on development of female reproductive tract and uterine carcinogenesis in Donryu rats. J Reprod Dev. 50, 349-360. 
Yoshihara S, Makishima M, Suzuki N, Ohta S. (2001). Metabolic activation of bisphenol A by rat liver S9 fraction. Toxicol. Sci. 62, 221-227.

Yoshihara S, Mizutare T, Makishima M, Suzuki N, Fujimoto N, Igarashi K, and Ohta S (2004) Potent estrogenic metabolites of bisphenol A and bisphenol B formed by rat liver S9 fraction: their structures and estrogenic potency. Toxicol Sci 78, 50-59.

You L, Zhu X, Shrubsole MJ, Fan H, Chen J, Dong J, Hao CM, Dai Q. (2011). Renal function, bisphenol $A$, and alkylphenols: results from the National Health and Nutrition Examination Survey (NHANES 2003-2006). Environ Health Perspect. 119, 527-533.

Zalko D, Jacques C, Duplan H, Bruel S and Perdu E (2011) Viable skin efficiently absorbs and metabolizes bisphenol A. Chemosphere. 82, 424-430.

Zhang $H$, Yamada H, Tsuno H. (2008). Removal of endocrine-disrupting chemicals during ozonation of municipal sewage with brominated byproducts control. Environ Sci Technol. $42,3375-3380$.

Zhang J., Cooke G.M., Curran I.H.A., Goodyer C.G., Cao X.-L. (2011) GC-MS analysis of bisphenol $A$ in human placental and fetal liver samples Journal of Chromatography $B, 879$ (2011) 209-214.

Zhu R, Zhao W, Zhai M, Wei F, Cai Z Sheng N and Hu Q (2010) Molecularly imprinted layer-coated silica nanoparticles for selective solid-phase extraction of bisphenol A from chemical cleansing and cosmetics samples. Anal Chim Acta 658: 209-216.

Zimmerman-Downs JM, Shuman D, Stull SC, Ratzlaff RE. (2010) Bisphenol A blood and saliva levels prior to and after dental sealant placement in adults. Journal of Dental Hygiene 2010;84: 145-50. 


\section{Annex I}

Eucomed comments on the use of BPA in Medical Devices (2012) Response to the SCENI HR call for information on BPA in medical devices

A brief survey of our members identified the following examples of device types with materials derived from BPA:

- Catheters for minimally invasive surgery

- Catheters for cardiac ablation

- Trocars

- Surgical and cardiovascular instruments

- Surgical meshes

- Laparoscopic instruments

- Endoscopes

- Spine cement mixing/delivery systems

- Polycarbonate packaging for products that are steam sterilized

- Photopheresis devices for cancer treatment

- Pacemakers

- Pacemaker catheter systems/catheter delivery systems

- Stents

- Stent delivery systems

- Blood oxygenator and dialysis membrane housings

- Luer fittings

- Needle hub

- Insulin Pump Infusion Sets

- Neurostimulators (implantable)

- Drug pumPS (implantable)

- Neuromodulation catheter system/catheter delivery systems

- Balloon Dilatation Catheters

Polycarbonate medical devices reported in Beronius and Hanberg 2011 (Plastic Europe 2007)

Blood oxygenators

Cardiotomy reservoirs

Dialysers

Respirators

Dentists' operating lamPS

Safety valves for respirators

Breast pumPS 


\begin{abstract}
Inhaler housings
Prescription spectacles

i.v. connectors

Scalpel cases

Laparoscope handles

Contact lens holders

Syringe toPS

Medical packaging film

\section{Ampoules}

Three-way stop cocks and stop cocks Manifolds

Tweezers with integrated lighting

Single-use operating instruments

Polycarbonate medical devices include:

- Catheters for minimally invasive surgery

- Catheters for cardiac ablation

- Trocars

- Surgical and cardiovascular instruments

- Surgical meshes

- Laparoscopic instruments

- Endoscopes

- Spine cement mixing/delivery systems

- Polycarbonate packaging for products that are steam sterilized

- Photopheresis devices for cancer treatment

- Pacemakers

- Pacemaker catheter systems/catheter delivery systems

- Stents

- Stent delivery systems

- Blood oxygenator and dialysis membrane housings

- Luer fittings

- Needle hubs

- Neurostimulators (implantable)

- Drug pumPS (implantable)

- Neuromodulation catheter system/catheter delivery systems

- Balloon Dilatation Catheters

(Information provided by Eucomed, 2012) 


\section{Annex I I}

\section{Summary of BPA concentrations in or released by medical devices / medical grade materials.}

Chromatographic methods are considered more suitable for BPA analysis in biological samples than immunochemical methods. ELISA methods are less reliable due to possible cross reactivity of BPA parent compound and the conjugated metabolites and other BPA similar phenolic structures, what can lead to an overestimation of free BPA concentrations; also their sensitivity is low and may be subjected to matrix effects (especially in urine samples).

Methods based on mass spectrometry detection (MS) are the most reliable. MS-MS detection provides high sensitivity and selectivity, allowing the confirmation of the identity of BPA based on fragment formations.

A crucial issue in BPA analysis at low levels in human samples, and a potential source of different results published in the literature, is the differentiation in the measurement of free BPA and its metabolites. Additionally, the contamination with BPA from external sources, during sample collection and processing in the laboratory has to be assessed through the analysis of blank samples to guarantee the reliability of the results. Moreover, the possible hydrolysis of the conjugated metabolites into BPA free form during sample storage needs to be considered. In that sense, those methods using an internal standard, typically a stable isotope labeled BPA with use of MS, are more reliable since they provide a means to determine the effect of complex matrices of blood and urine samples, and the stability of BPA in the biological samples during analysis. 
BPA in Medical Devices

\begin{tabular}{|c|c|c|c|c|c|c|c|}
\hline Reference & Sample & $\begin{array}{l}\text { Extraction } \\
\text { method }\end{array}$ & $\begin{array}{l}\text { Method of } \\
\text { analysis }\end{array}$ & LOD & $\begin{array}{c}\text { BPA } \\
\text { concentration }\end{array}$ & Remarks & Reliability \\
\hline $\begin{array}{l}\text { Haishima } \\
\text { et al. } \\
\text { (2001) }\end{array}$ & $\begin{array}{l}\text { PC pellets (2 } \\
\text { types) } \\
\text { PS pellets } \\
\text { PC } \\
\text { casings(2) } \\
\text { (lacking } \\
\text { hollow } \\
\text { fibers) }\end{array}$ & $\begin{array}{l}\text { Dissolution } \\
\text {, THF } \\
\\
\text { Extraction } \\
\text { (shaking, } \\
16 \mathrm{~h}, \mathrm{RT} \text {, } \\
10 \mathrm{~mL} \text { ): } \\
\text { water } \\
\text { methanol }\end{array}$ & $\begin{array}{l}\text { HPLC } \\
\text { GC-MS } \\
\text { LC-MS, } \\
\text { and NMR } \\
\text { spectrosco } \\
\text { py }\end{array}$ & $\begin{array}{l}0.65 \mathrm{ppb} \\
\text { (HPLC) } \\
0.16 \mathrm{ppb} \\
\text { (GC-MS) }\end{array}$ & $\begin{array}{l}4.0 \text { and } 7.2 \\
\text { ug/g } \\
34.5 \text { ug/g } \\
\\
11.7 \text { and } 13.7 \\
\text { ng/casing } \\
296 \text { and } 345 \\
\text { ng/casing }\end{array}$ & $\begin{array}{l}\text { Total } \\
\text { content in } \\
\text { the } \\
\text { material. } \\
\\
\text { Released } \\
\text { amount } \\
\text { from the } \\
\text { material } \\
\text { under the } \\
\text { indicated } \\
\text { conditions }\end{array}$ & + \\
\hline $\begin{array}{l}\text { Data } \\
\text { submitted } \\
\text { in } \\
\text { response } \\
\text { to the Call } \\
\text { for } \\
\text { Informatio } \\
\text { n (2012) }\end{array}$ & $\begin{array}{l}\text { PC pellets } \\
\text { PC trocar } \\
\text { tubing }\end{array}$ & $\begin{array}{l}\text { Extraction, } \\
37^{\circ} \mathrm{C}, 24 \mathrm{~h}: \\
\text { IP } \\
\text { Ethanol } \\
\\
\text { IP } \\
\text { Ethanol } \\
\\
\\
0.9 \% \\
\text { sodium } \\
\text { chloride, } \\
37^{\circ} \mathrm{Ctime} \\
\text { pointup to } \\
168 \mathrm{~h}\end{array}$ & HPLC & & $\begin{array}{l}0.247-<0.52 \\
u g / g \\
<0.5 \mathrm{ug} / \mathrm{g} \\
0.28-<0.50 \\
\mathrm{ug} / \mathrm{g} \\
<0.5 \mathrm{ug} / \mathrm{g} \\
<2 \mathrm{ug} / \mathrm{g}\end{array}$ & $\begin{array}{l}\text { Total } \\
\text { content } \\
\text { extracted } \\
\text { with IP } \\
\text { according } \\
\text { to the } \\
\text { authors } \\
\text { No } \\
\text { detailed } \\
\text { informati } \\
\text { on on } \\
\text { method } \\
\text { performa } \\
\text { nce to } \\
\text { assess } \\
\text { the } \\
\text { reliability } \\
\text { of the } \\
\text { data }\end{array}$ & + \\
\hline $\begin{array}{l}\text { Haishima } \\
\text { et al. } \\
\text { (2001) }\end{array}$ & $\begin{array}{l}\text { Hemodialyze } \\
\text { rs: } \\
\text { PCcasing/ PS } \\
\text { fiber (2) } \\
\text { PCcasing/ } \\
\text { cellulose } \\
\text { acetate } \\
\text { fiber (1) } \\
\text { Polystyrene } \\
\text { casing/PS } \\
\text { fiber (1) }\end{array}$ & 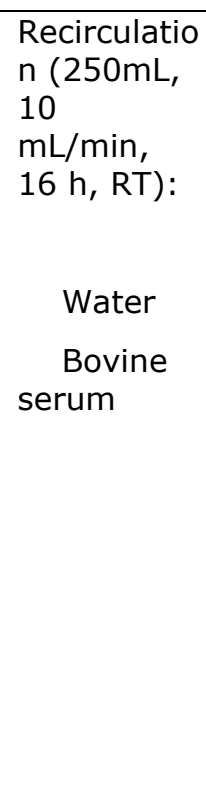 & $\begin{array}{l}\text { HPLC } \\
\text { GC-MS } \\
\text { LC-MS, } \\
\text { and NMR } \\
\text { spectrosco } \\
\text { py }\end{array}$ & $\begin{array}{l}0.65 \mathrm{ppb} \\
\text { (HPLC) } \\
0.16 \mathrm{ppb} \\
\text { (GC-MS) }\end{array}$ & $\begin{array}{l}\text { Water } \\
\text { Bovine serum } \\
\text { (ng/module) } \\
\\
31.0-141.8 \\
1010-2090 \\
\\
\\
34.1 \\
196.1 \\
\\
3.78 \\
140.7\end{array}$ & $\begin{array}{l}\text { Released } \\
\text { amount } \\
\text { from the } \\
\text { material } \\
\text { under the } \\
\text { indicated } \\
\text { conditions } \\
\cdot \\
\text { Higher } \\
\text { BPA } \\
\text { released } \\
\text { was } \\
\text { found for } \\
\text { all types } \\
\text { in bovine } \\
\text { serum } \\
\text { compared } \\
\text { with } \\
\text { water }\end{array}$ & + \\
\hline $\begin{array}{l}\text { Shintani } \\
\text { (2001) }\end{array}$ & $\begin{array}{l}\text { Hemodialyze } \\
\text { rs: }\end{array}$ & $\begin{array}{l}\text { Perfusion } \\
\text { on } \\
\text { patients, } 4 \\
\text { h, } 3 \text { times }\end{array}$ & $\begin{array}{l}\text { HPLC-ECD } \\
\text { HPLC-MS }\end{array}$ & $\begin{array}{l}0.02 \\
\mathrm{ng} / \mathrm{mL} \\
\text { plasma }\end{array}$ & & $\begin{array}{l}\text { Measured } \\
\text { values in } \\
\text { blood of } \\
\text { uremia }\end{array}$ & $\begin{array}{l}\text { Not reliable } \\
\text { as the } \\
\text { sensitivity } \\
\text { and }\end{array}$ \\
\hline
\end{tabular}




\begin{tabular}{|c|c|c|c|c|c|c|c|}
\hline & $\begin{array}{l}\text { PC casing/ } \\
\text { PS fiber ) } \\
\text { autoclaving } \\
\text { sterilization } \\
\text { (2) } \\
\text { PCcasing/ } \\
\text { PS fiber - } \\
\text { gamma-ray } \\
\text { sterilization } \\
\text { (1) } \\
\text { Polystyrene- } \\
\text { butadiene } \\
\text { coplymer } \\
\text { casing/PS } \\
\text { fiber- } \\
\text { gamma-ray } \\
\text { sterilization( } \\
\text { 1) } \\
\text { PCcasing/ } \\
\text { PS fiber )- } \\
\text { autoclaving } \\
\text { sterilization } \\
\text { (2) }\end{array}$ & $\begin{array}{l}\text { a week, for } \\
3 \text { months } \\
\text { consecutiv } \\
\text { ely } \\
\\
\\
\text { Saline } \\
\text { solution - } \\
800 \mathrm{~mL} \\
\text { (according } \\
\text { to ISO } \\
10993-7 \text { ) }\end{array}$ & & & $\begin{array}{l}0.2-0.7 \\
\mathrm{ng} / \mathrm{mL} \text { (mean } \\
\text { value, } \mathrm{n}=4) \\
<\text { LOD (mean } \\
\text { value, } \mathrm{n}=4) \\
<\text { LOD (mean } \\
\text { value, } \mathrm{n}=4 \text { ) } \\
0.1-0.2 \mathrm{ng} / \mathrm{mL}\end{array}$ & $\begin{array}{l}\text { patients } \\
\text { after } \\
\text { dialysis. } \\
\text { No BPA } \\
\text { was } \\
\text { detected } \\
\text { in the the } \\
\text { blood } \\
\text { samples } \\
\text { collected } \\
\text { before } \\
\text { dialysis }\end{array}$ & $\begin{array}{l}\text { specificity } \\
\text { of the } \\
\text { method is } \\
\text { insufficient } \\
\text { to detect } \\
\text { and } \\
\text { quantify the } \\
\text { concentratio } \\
\text { ns in serum } \\
\text { of normal } \\
\text { subjects. } \\
\text { The value of } \\
0.2-0.7 \\
\text { ng/ml is not } \\
\text { reliable. }\end{array}$ \\
\hline $\begin{array}{l}\text { Yamasaki } \\
\text { et al. } \\
\text { (2001) }\end{array}$ & $\begin{array}{l}\text { Hemodializer } \\
\text { S: } \\
\text { Polystyrene } \\
\text { casing/PS } \\
\text { fiber }(A, B) \\
\text { PCcasing/ } \\
\text { PS fiber } \\
\text { (C,D) } \\
\text { PCcasing/ } \\
\text { EVAL fiber } \\
\text { (E) }\end{array}$ & $\begin{array}{l}\text { Filled with } \\
\text { reverse } \\
\text { osmotic } \\
\text { water. } \\
\\
\text { Recirculati } \\
\text { on of } \\
\text { saline } \\
\text { solution } \\
(200 \mathrm{~mL} \text { ), } \\
200 \\
\mathrm{~mL} / \mathrm{min}, \\
4 \mathrm{~h} \\
\\
\text { Blood } \\
\text { samples } \\
\text { from } \\
\text { hemodialy } \\
\text { zed } \\
\text { patients } \\
\text { (dialysate } \\
\text { flow rate } \\
500 \\
\mathrm{~mL} / \mathrm{min} \text { ) }\end{array}$ & HPLC & Not given & $\begin{array}{l}\text { ND }(A, B) \\
0.23 \mathrm{ppb}(C), \\
1.14 \mathrm{ppb}(\mathrm{D}) ; \\
0.19 \mathrm{ppb}(\mathrm{E}) \\
\mathrm{ND}(\mathrm{A}, \mathrm{B}) \\
0.16 \mathrm{ppb}(\mathrm{C}), \\
0.75 \mathrm{ppb}(\mathrm{D}) ; \\
0.20 \mathrm{ppb}(\mathrm{E}) \\
\mathrm{ND}(\mathrm{C})(\mathrm{n}=3) \\
0.49 ; 0.67: \\
1.15 \mathrm{ppb}(\mathrm{D})\end{array}$ & $\begin{array}{l}\text { Lack of } \\
\text { data on } \\
\text { method } \\
\text { performa } \\
\text { nce. } \\
\text { Released } \\
\text { BPA } \\
\text { amount } \\
\text { from the } \\
\text { material } \\
\text { under the } \\
\text { indicated } \\
\text { conditions } \\
\text { Saline } \\
\text { solution } \\
\text { was } \\
\text { recirculat } \\
\text { ed after } \\
\text { the } \\
\text { removal } \\
\text { of the } \\
\text { osmotic } \\
\text { water and } \\
\text { washing } \\
\text { with } 800 \\
\text { mL of } \\
\text { saline } \\
\text { solution } \\
\\
\text { Mean }\end{array}$ & not reliable \\
\hline
\end{tabular}




\begin{tabular}{|c|c|c|c|c|c|c|c|}
\hline & & & & & & $\begin{array}{l}\text { value } \\
0.77 \mathrm{ppb} \\
\text { in blood. } \\
\text { Patients } \\
\text { with } \\
\text { almost no } \\
\text { residual } \\
\text { renal } \\
\text { function }\end{array}$ & \\
\hline $\begin{array}{l}\text { Shintani et } \\
\text { al. (2003) }\end{array}$ & $\begin{array}{l}\text { Membranes, } \\
\text { ozone gas } \\
\text { sterilized: } \\
\text { PS, } 20 \text { mg } \\
\text { aprox. (3) } \\
\text { PC, } 7 \text { mg } \\
\text { aprox. (1) } \\
\text { Both types } \\
\text { unsterilised } \\
\text { and steam- } \\
\text { sterilized } \\
\text { (121C, } 15 \\
\text { min) }\end{array}$ & $\begin{array}{l}\text { Extraction, } \\
\text { ethanol }\end{array}$ & LC-UV-MS & $0.02 \mathrm{ppb}$ & $\begin{array}{l}43 \text { - } 207 \mathrm{mg} / \mathrm{kg} \\
\text { ozone gas } \\
\text { sterilized: } \\
119 \mathrm{ppm} \\
<\text { LOD }\end{array}$ & $\begin{array}{l}\text { Released } \\
\text { amount } \\
\text { from the } \\
\text { material } \\
\text { under the } \\
\text { indicated } \\
\text { conditions } \\
\text {. } \\
\text { BPS also } \\
\text { detected } \\
\text { in PS } \\
\text { membran } \\
\text { es ( } 355 ; \\
63 ; 32 \\
\text { mg/kg) }\end{array}$ & reliable \\
\hline $\begin{array}{l}\text { Murakami } \\
\text { et } \\
\text { al. }(2007)\end{array}$ & $\begin{array}{l}\text { Hollow } \\
\text { fibers: } \\
\text { PS } \\
\text { PEPA } \\
\text { (Polyester- } \\
\text { polymeralloy } \\
\text { ) } \\
\text { Hemodialyze } \\
\text { rs with PS } \\
\text { membranes }\end{array}$ & $\begin{array}{l}\text { Extraction } \\
\text { (10 mg) } \\
\text { with } \\
\text { hexane. } \\
\text { Redisolutio } \\
\text { n of } \\
\text { residue in } \\
\text { dimethylsul } \\
\text { foxide. } \\
\\
\\
\text { Perfusion } \\
\text { on } 15 \\
\text { patients, } \\
\text { who } \\
\text { previously } \\
\text { underwent } \\
\text { hemodialys } \\
\text { is for at } \\
\text { least three } \\
\text { months } \\
\text { with a PS } \\
\text { dyalizer }\end{array}$ & ELISA & $\begin{array}{l}0.3 \\
\mathrm{ng} / \mathrm{mL}\end{array}$ & $\begin{array}{l}83.3 \mathrm{ng} / 10 \mathrm{mg} \\
122.5 \mathrm{ng} / 10 \mathrm{mg} \\
\text { Mean increase, } \\
\text { after } 1 \text { month } \\
\text { session: } \\
\text { from } 4.83 \pm 1.94 \\
\text { to } 6.62 \pm 3.09 \\
\text { ng/mL ( } 1^{\text {st }} \text { test) } \\
\text { from } 4.09 \pm \\
2.78 \text { to } \\
4,27 \pm 2.98 \\
\text { ng/mL ( } 2^{\text {nd }} \text { test) }\end{array}$ & $\begin{array}{l}\text { Released } \\
\text { amount } \\
\text { from the } \\
\text { material } \\
\text { under the } \\
\text { indicated } \\
\text { conditions } \\
\text { (mean } \\
\text { values, } \\
\text { n=6) } \\
\\
\text { The } \\
\text { quoted } \\
\text { difference } \\
\text { s are } \\
\text { certainly } \\
\text { not } \\
\text { statisticall } \\
\text { y } \\
\text { significan } \\
\text { t. } \\
56.6 \% \\
\text { cross- } \\
\text { reactivity } \\
\text { with } \\
\text { water- } \\
\text { soluble } \\
\text { form of } \\
\text { BPA. }\end{array}$ & $\begin{array}{l}\text { not reliable, } \\
\text { because of } \\
\text { cross- } \\
\text { reactivity }\end{array}$ \\
\hline $\begin{array}{l}\text { Fink } \\
(2008)\end{array}$ & $\begin{array}{l}\text { Dyalizers } \\
\text { with PS or } \\
\text { PC (5) } \\
\text { Surface area }\end{array}$ & $\begin{array}{l}\text { Simulated } \\
\text { dyalisis, } 37 \\
{ }^{\circ} \mathrm{C}, 230 \mathrm{~mL} \\
\text { eluate/min } \\
\text { for } 4 \mathrm{~h} \text { and } \\
24 \mathrm{~h} \text { as }\end{array}$ & LC-MS-MS & & & $\begin{array}{l}\text { Range of } \\
\text { BPA } \\
\text { amount } \\
\text { eluted } \\
\text { from the } \\
\text { dialysers }\end{array}$ & reliable \\
\hline
\end{tabular}




\begin{tabular}{|c|c|c|c|c|c|c|}
\hline & $(1.3-1.8 \mathrm{~m} 2)$ & $\begin{array}{l}\text { worst case } \\
\text { scenario } \\
\text { reverse } \\
\text { osmotic } \\
\text { water } \\
17.2 \% \\
\text { ethanol }\end{array}$ & & $\begin{array}{l}6.4-71.3 \\
\text { ng/dialyzer } \\
54.8-4299 \\
\text { ng/dialyzer }\end{array}$ & $\begin{array}{l}\text { in a } \\
\text { period } \\
\text { time from } \\
4 \text { to } 24 \mathrm{~h} \text {. } \\
\text { BPA } \\
\text { leaching } \\
\text { increased } \\
\text { with the } \\
\text { membran } \\
\text { e surface } \\
\text { area and } \\
\text { with } \\
\text { dialysis } \\
\text { time. }\end{array}$ & \\
\hline $\begin{array}{l}\text { Krieter et } \\
\text { al. (2013) }\end{array}$ & $\begin{array}{l}\text { Hemodializer } \\
\mathrm{s:} \\
1.3 \mathrm{~m}^{2} \\
\text { polysulfone } \\
\text { membranes, } \\
\text { high flux } \\
1.3 \mathrm{~m}^{2} \\
\text { polysulfone } \\
\text { membranes, } \\
\text { low flux. } \\
1.7 \text { m2 } \\
\text { high-flux } \\
\text { polyethersulf } \\
\text { one } \\
\text { membrane }\end{array}$ & $\begin{array}{l}\text { Recirculatio } \\
\mathrm{n} \text { of sterile } \\
\text { water (400 } \\
\mathrm{mL}), 3 \mathrm{~h}, \\
250 \\
\mathrm{ml} / \mathrm{min}, 37 \\
{ }^{\circ} \mathrm{C}\end{array}$ & ELISA & $\begin{array}{l}\text { Total amount } \\
\text { ng/dialyzer, } \\
\text { mean values } \\
(n=6) \text { : } \\
48.1 \pm 7.7 \\
140.8 \pm 38.7 \\
6.2 \pm 2.5\end{array}$ & $\begin{array}{l}\text { BPA } \\
\text { eluted } \\
\text { under the } \\
\text { indicated } \\
\text { conditions } \\
\end{array}$ & $\begin{array}{l}\text { not reliable } \\
\text { because of } \\
\text { unspecific } \\
\text { analytical } \\
\text { method }\end{array}$ \\
\hline & & $\begin{array}{l}\text { Perfusion } \\
\text { on patients } \\
(18), 4 \\
\text { weektreat } \\
\text { ment }\end{array}$ & & $\begin{array}{l}\text { No significant } \\
\text { plasma levels } \\
\text { changes after } \\
\text { treatment with } \\
\text { any of dialyzers }\end{array}$ & $\begin{array}{l}\text { BPA } \\
\text { plasma } \\
\text { levels in } \\
\text { the } \\
\text { control } \\
\text { group } \\
\text { ( } \mathrm{n}=24) \\
\text { were } \\
\text { significan } \\
\text { t lower( } \\
\leq 0.2 \\
\pm 0.1 \\
\text { ng/mL) } \\
\text { compared } \\
\text { to pre- } \\
\text { dialysis } \\
\text { values of } \\
\text { patients } \\
\text { (9.1 } \pm \\
4.5- \\
12.0 \pm \\
6.0 \\
\text { ng/mL). } \\
\text { Lack of } \\
\text { data on }\end{array}$ & \\
\hline
\end{tabular}




\begin{tabular}{|c|c|c|c|c|c|c|c|}
\hline & & & & & & $\begin{array}{l}\text { method } \\
\text { performa } \\
\text { nce. }\end{array}$ & \\
\hline $\begin{array}{l}\text { Cho et al. } \\
(2012)\end{array}$ & $\begin{array}{l}\text { Hemodialyze } \\
\text { r/hemoconce } \\
\text { ntrators with } \\
\text { polysulfone } \\
\text { membranes: } \\
0.5 \mathrm{~m}^{2}(7.9 \\
\text { g) } \\
0.4 \mathrm{~m}^{2} \\
0.7 \mathrm{~m}^{2}\end{array}$ & $\begin{array}{l}\text { Ten } \\
\text { consecutiv } \\
\text { e } \\
\text { extractions } \\
\text { ( } 1 \text { h each) } \\
\text { with } 1 \mathrm{~L} \text { of } \\
17.2 \% \\
\text { ethanol at } \\
37^{\circ} \mathrm{C}, 200 \\
\text { mL/min. } \\
\text { One single } \\
\text { extraction, } \\
6 \mathrm{~h} \text {, with } \\
1 \mathrm{~L} \text { of } 17.2 \\
\% \text { ethanol } \\
\text { at } 37^{\circ} \mathrm{C}, \\
200 \\
\text { mL/min. }\end{array}$ & LC/MS & $\begin{array}{l}0.02 \\
\mathrm{ng} / \mathrm{ml} \\
(\mathrm{LOQ})\end{array}$ & $\begin{array}{l}19.7 \mathrm{ug} \\
\approx 1.3-1.4 \mathrm{ng} / \mathrm{ml} \\
\approx 0.65-0.7 \\
\mathrm{ng} / \mathrm{ml}\end{array}$ & $\begin{array}{l}\text { The } \\
\text { released } \\
\text { amount } \\
\text { from the } \\
\text { material } \\
\text { correspon } \\
\text { ded to 95 } \\
\text { \% of the } \\
\text { predicted } \\
\text { amount } \\
\text { using an } \\
\text { elution } \\
\text { profile } \\
\text { equation. } \\
\\
\text { Aproxima } \\
\text { ted } \\
\text { values } \\
\text { read from } \\
\text { the } \\
\text { published } \\
\text { figure. } \\
\text { Limited } \\
\text { informati } \\
\text { on on } \\
\text { method } \\
\text { performa } \\
\text { nce. }\end{array}$ & reliable \\
\hline $\begin{array}{l}\text { data } \\
\text { submitted } \\
\text { in } \\
\text { response } \\
\text { to the Call } \\
\text { for } \\
\text { Informatio } \\
\text { n (2012) }\end{array}$ & $\begin{array}{l}\text { Neonatal } \\
\text { incubators }\end{array}$ & $\begin{array}{l}\text { Analysis of } \\
\text { gas in } \\
\text { contact } \\
\text { with the } \\
\text { hoods }\end{array}$ & $\begin{array}{l}\text { ISO } 16000- \\
6\end{array}$ & $50 \mathrm{ug} / \mathrm{m} 3$ & $<$ LOD & $\begin{array}{l}\text { Very } \\
\text { limited } \\
\text { informati } \\
\text { on } \\
\text { provided } \\
\text { to assess } \\
\text { the } \\
\text { reliability } \\
\text { of the } \\
\text { data }\end{array}$ & unknown \\
\hline $\begin{array}{l}\text { Sakurai H. } \\
(2002) \\
\text { only } \\
\text { abstract in } \\
\text { english }\end{array}$ & $\begin{array}{l}\text { Cardiopulmo } \\
\text { nary bypass } \\
\text { circuits with } \\
\text { PC parts (8) }\end{array}$ & $\begin{array}{l}\text { Priming } \\
\text { with a } \\
\text { saline } \\
\text { solution } \\
\\
\text { Blood } \\
\text { samples } \\
\text { taken from } \\
6 \text { patients } \\
\text { once the } \\
\text { CBP was }\end{array}$ & GC & & $\begin{array}{l}0.9 \pm 1.1 \mathrm{ug} / \mathrm{l} \\
0.3 \pm 0.2 \mathrm{ug} / \mathrm{l} \\
\text { (after the } \\
\text { commencement } \\
\text { of CBP) } \\
0.4 \pm 0.3 \mathrm{ug} / \mathrm{l} \\
\text { (at the }\end{array}$ & $\begin{array}{l}\text { No BPA } \\
\text { detected } \\
\text { in } 3 \\
\text { controls } \\
\text { samples. } \\
\text { Info in } \\
\text { the } \\
\text { abstract } \\
\text { not } \\
\text { enough } \\
\text { for the } \\
\text { evaluatio } \\
\text { n of the } \\
\text { data. }\end{array}$ & unknown \\
\hline
\end{tabular}




\begin{tabular}{|c|c|c|c|c|c|c|c|}
\hline & & $\begin{array}{l}\text { initiated } \\
\text { and at the } \\
\text { termination }\end{array}$ & & & $\begin{array}{l}\text { termination of } \\
\text { CBP) }\end{array}$ & $\begin{array}{l}\text { The } \\
\text { quoted } \\
\text { difference } \\
\text { s are } \\
\text { certainly } \\
\text { not } \\
\text { statisticall } \\
\text { y } \\
\text { significan } \\
\text { t. } \\
\text { Info in } \\
\text { the } \\
\text { abstract } \\
\text { not } \\
\text { enough to } \\
\text { assess } \\
\text { the } \\
\text { reliability } \\
\text { of the } \\
\text { data. }\end{array}$ & \\
\hline $\begin{array}{l}\text { Lewis et al. } \\
\text { (1999) }\end{array}$ & $\begin{array}{l}\text { Commerciall } \\
\text { y available } \\
\text { dental } \\
\text { composites } \\
\text { and sealants }\end{array}$ & $\begin{array}{l}\text { Dissolution } \\
\text { in } \\
\text { acetonitrile } \\
\text { (removal of } \\
\text { fillers by } \\
\text { centrifugati } \\
\text { on) }\end{array}$ & HPLC & No quant. & & $\begin{array}{l}\text { BPA not } \\
\text { verified in } \\
\text { any } \\
\text { material. } \\
\text { Bis-DMA } \\
\text { verified in } \\
3 \\
\text { products } \\
\text { in the } \\
\text { same } \\
\text { product } \\
\text { line }\end{array}$ & unknown \\
\hline $\begin{array}{l}\text { Manabe et } \\
\text { al. (2000) }\end{array}$ & $\begin{array}{l}\text { Commerciall } \\
\text { y available } \\
\text { dental } \\
\text { composites } \\
\text { and sealants }\end{array}$ & $\begin{array}{l}\text { Dissolution } \\
\text { in } \\
\text { methanol } \\
\text { (removal of } \\
\text { fillers by } \\
\text { centrifugati } \\
\text { on) }\end{array}$ & $\begin{array}{l}\text { Derivatizati } \\
\text { on w TMS } \\
\text { GC/MS }\end{array}$ & $1 \mathrm{ng} / \mathrm{mg}$ & $\begin{array}{l}6.6 / 15.4 / 18.5 / 2 \\
0.2 \mathrm{ng} / \mathrm{mg} \\
\text { material }\end{array}$ & $\begin{array}{l}\text { In } 4 \\
\text { materials } \\
\text { (un- } \\
\text { polymeriz } \\
\text { ed). Not } \\
\text { detected } \\
\text { in } 2 \\
\text { mater. }\end{array}$ & reliable \\
\hline $\begin{array}{l}\text { Mazzaoui } \\
\text { et } \\
\text { al. }(2002)\end{array}$ & $\begin{array}{l}\text { Different } \\
\text { dental } \\
\text { materials }\end{array}$ & $\begin{array}{l}\text { Extraction } \\
\text { into water } \\
\text { or } \\
\text { water/etha } \\
\text { nol mix }\end{array}$ & HPLC/MS & - & $\begin{array}{l}0 \text { from } 3 \\
\text { materials, } \\
13 \pm 8 \text { or } 67 \pm \\
4 \mathrm{mmol} / \mathrm{m}^{2}(1 \mathrm{~d}, \\
\text { water } \text { or } 122 \pm \\
18 \text { or } 399 \pm 61 \\
\mathrm{mmol} / \mathrm{m}^{2}(90 \mathrm{~d}, \\
75 \% \text { ethanol) }\end{array}$ & $\begin{array}{l}\text { BPA } \\
\text { extracted } \\
\text { from } 1 \\
\text { sealant } \\
\text { and } 1 \\
\text { bonding } \\
\text { material }\end{array}$ & reliable \\
\hline
\end{tabular}

